LIBRARY ANNEX

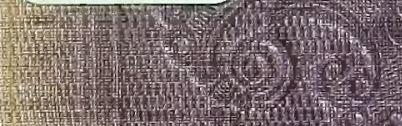

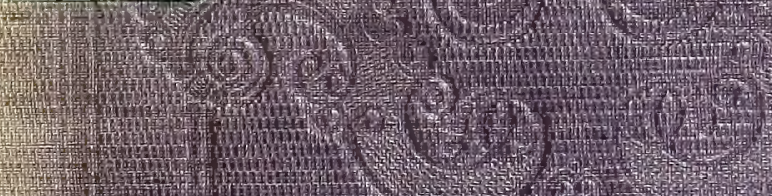

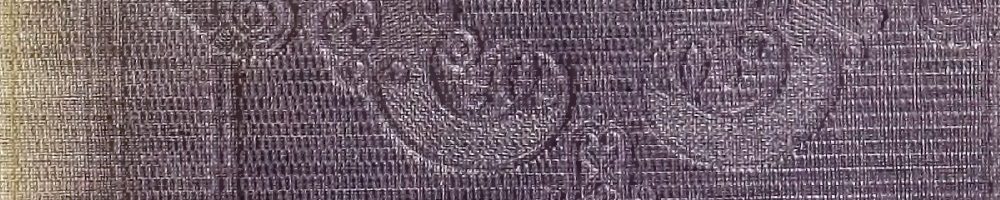
$-4$

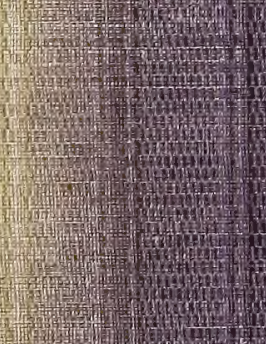
… … ㄴ. 4<smiles>C=[SiH]</smiles>
tint (1) (4)

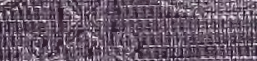


F R O M

\section{Clye $\mathfrak{k}$ cgents of the Hniversitu,}

EX-OFFICIO

TRUSTEES OF THE STATE LIBRARY,

IN BEHALF OF THE

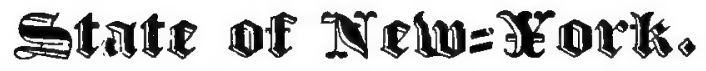

Oa

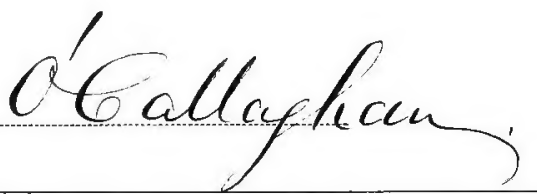




\section{Cornell University Library}

The original of this book is in the Cornell University Library.

There are no known copyright restrictions in the United States on the use of the text.

http://www.archive.org/details/cu31924024735429 



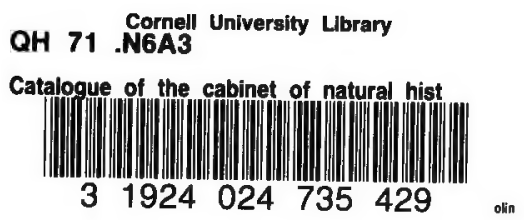





\title{
CATALOGUE
}

OF THE

\section{CABINET OF NATURAL HISTORY}

\section{OF THE STATE OF NEW.YORK,}

\author{
AND OF THF \\ 9. \\ ANNEXED THERETO. \\ Printed by Order of the Regents of the University. \\ A L B A N Y : \\ C. VAN BENTHUYSEN, PRINTER TO THE LEGISLATURE. \\ 1853 ,




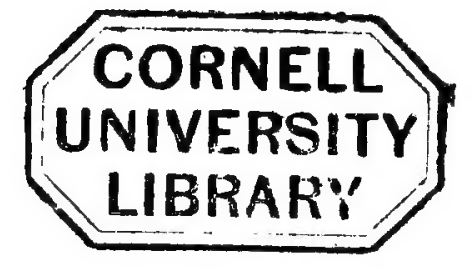




\section{REGENTS OF THE UNIVERSITY,1853.}

HORATIO SEYMOUR, Governor, ex officio.

SANFORD E. CHURCH, Lieutenant-Governor, ex officio.

HENRY S. RANDALL, Secretary of State, ex officio.

GERRIT Y. LANSING, Chancellor.

JOHN GREIG, Vice-Chancellor.

GULIAN C. VERPLANCK, LL.D. JOHN K. PAIGE.

ERASTUS CORNING.

PROSPER M. WETMORE.

JOHN L. GRAHAM.

JOHN M'LEAN.

GIDEON HAWLEY, LL.D.

DAVID BUEL.

JAMES S. WADSWORTH.
JOHN V. L. PRUYN, LL.D. JABEZ D. HAMMOND, LL.D. JOHN L. O'SULLIVAN. ROBERT CAMPBELL.

Rev. SAMUEL LUCKEY, D.D. ROBERT G. RANKIN. PHILIP S. VAN RENSSELAER. Rev. JOHN N. CAMPBELL, D.D.

T. ROMEYN BECK, Secretary.

\section{STANDING COMMITTEE OF THE REGENTS,}

SPECIALLY CHARGED WITH THE CARE OF THB STATE CABINET.

HORATIO SEYMOUR, Governor.

HENRY S. RANDALL, Secretary of State.

ERASTUS OORNING.

JOHN M'LEAN.

JAMES S. WADSWORTH.

JOHN GEBHARD JUNIoR, Curator. JAMES HURST, Taxidermist. 



\section{MEMORANDUM.}

THE Regents of the University have directed the publication of the accompanying Catalogue, with the double object of showing the contents and the wants of the Collection.

When received under their charge in 1848 , it was found to be in an extremely imperfect condition; and most, indeed, of the quadrupeds and birds have been replaced by better specimens.

As the Catalogue is intended as an accompaniment to the "Natural History of New-York," the nomenclature there used has been preserved.

A portion only of the Collection is contained in this volume. The Fishes, Insects, Shells, etc. are for the present omitted, in the hope that they may soon be increased in number, and duly arranged and named. The various divisions are accordingly so paged, that additions can be readily made, and new parts inserted. The attention of Naturalists is invited to the deficiencies in the Cabinet, in the hope that, through their aid and advice, these may be supplied.

The building at present appropriated for this Collection has beeome of too small capacity for properly exhibiting its contents; and the Regents can only rely on the liberality of the Legislature, to furnish them with accommodations worthy of what in a few years may be made a Museum of the animal, vegetable, and mineral products of the State of New-York. T. R. B. 



\section{CONTENTS,}

ZOOLOGY :

Part i. Mammalia

Part II. BIRDS

Parts iII and iv. Reptiles ANd Amphibia

BOTANY

MINERALOGY

GEOLOGY

PALAONTOLOGY

HISTORICAL AND ANTIQUARIAN OOLLECTION

COLLECTION OF THE LATE H. C. DE RHAM JUNIOR
8 pages. pages 15 to 28. pages 29 to 32 .

61 pages.

- 22 pages.

53 pages.

- 31 pages.

28 pages.

21 pages. 



\section{Z00LOGY OF NEW-YORK,}

OR THE

$\mathfrak{N} \mathfrak{e} \mathfrak{w}-\mathbb{1} \mathfrak{o} \mathfrak{x} \mathfrak{l} \quad \mathfrak{f} \mathfrak{a} \mathfrak{a} \mathfrak{n} \mathfrak{a}$

BY JAMES E. DE KAY.

PART I. MAMMaLIA. 
N. B. If there be a specimen of any animal named in the Collection, it is so stated in the third column. 


\section{Z $00 \mathrm{~L} 0 \mathrm{GY}$.}

\section{CLASS I. MAMMALIA.}

\section{ORDER I. QUADRUMANA.}

ORDER II. MARSUPIATA.

FAMILY DIDELPHID疋.

Latin Name.

English Name.

In the

1 Didelyhis virginiana, American Opossum. - - Cabinet.

\section{ORDER III. CARNIVORA.}

\section{FAMILY VESPERTILIONID AS.}

2 Vespertilio noveboracensis, Neu-York Bat (m. \& f.). - Cabinet.

3 Vespertilio pruinosus, Hoary Bat.

4 Vespertilio subulatus, Little Brown Bat. - - - Cabinet.

5 Vespertilio noctivagans, Silver-haired Bat (m. \& f.). Cabinet.

6 Vespertilio carolinensis, Carolina Bat (m. f.\& y.). - Cabinet.

FAMILY SORECID无.

7 Condylura cristata, Common Starnose (two). - Cabinet.

8 Scalops aqdaticus, Common Shrew Mole. - - Cabinet.

9 Sorex dekayi, Dekay's Shrew. - - - Cabinet.

10 Sorex Brevicaudus, Short-tailed Shrew.

11 Sorex parvos, Small Shrew.

12 Sorex forsteri, Forster's Shrevi.

13 Sorex carolinensis, Carolina Shrew.

14 Otisorex platyrhinus, Broad-nosed Shrew. 
FAMILY URSID AE.

Latin Names.

15 Ursus americanus,

16 Procyon lotor,

17 Gulo Luscus,
English Names.

American Black Bear. - - Cabinet.

Raccoon (m. \& f.). - - - Cabinet.

Wolverene.

FAMILY MUSTELID A.

18 Mephitis americana,

Skunk. - - - - - - Cabinet.

19 Mustela canadensis, Fisher. - - - - - - Cabinet.

20 Mustela martes,

American Sable.

21 Mustela pusilla,

Small Weasel.

22 Mustela ftrsca,

Brown Weasel.

23 Putorius noveboracensis, New-York Ermine (m. \& f.). Cabinet.

24 Putorius vison, Mink. - - - - - Cabinet.

FAMILY LUTRID坧.

20 Lutra canadensis,

North-American Otter. - - Cabinet.

FAMILY CANID王.

26 CANis Familiaris,

Dornestic Dog (domesticated).

27 Lutupus occidentalis,

Common American Wolf.

28 Vulpes Fulvus,

29 Vulpes virginiants,

Red Fox. - - - - - - Cabinet.

Grey Fox. - - - . - - Cabinet.

FAMILY FELID㤅.

30 Felis concolor,

31 Lyncus Borealis,

32 LyNCUS RUFUs,

Northern Panther (2 m. \& f.). Cabinet.

Northern Lynx. - - - - Cabinet.

Wildcat, or Bay Lynx. - - Cabinet.

FAMILY PHOCID无.

33 Phoca concolor,

American Seal. - - - - Cabinct.

34 Stemmatopus cristatus, Hooded Seal.

\section{ORDER IV. RODENTIA.}

FAMILY SOIURID E.

35 Sciurus leucotis,

36 Sciurus vulpinus,

37 Scturus Niger,

38 Sciurus hudsonicus,

39 Sciurus struatus, 40 Pteromys volucelia, Small American Flying siquirrel. (m. \& f.). Cabinet.

Little Grey Squirrel. - - Cabinet.

Fox Squirrel (m. \& f.). - - Cabinet.

Black Squirrel. - - - Cabinet.

Red Squirrel (m. \& f.). - - Cabinet.

Striped Squirrel. - . - - 
FAMILY ARCTOMIDA.

Latin Names.

41 Arctomys monax,
English Names.

Wogdchuck (m. \& f.). - - Cabinet.

FAMILY GERBILLIDAE.

42 Meriones americanus, Deer Mouse (f.). - - - - Cabinet. FAMILY CASTORID $A$.

43 Castor fiber, 44 Fiber zibenthicus, Beaver. - - - - - - Cabinet. Musquash (2 m. \& f.). - - Cabinet.

FAMILY HYSTRICID $A$.

45 Hystrix hudsonius,

North-American Porcupine(f.). Cabinet.

FAMILY MURIDA.

46 Mus decumanus, 47 Mus rattus,

48 Mus americants,

49 Mus musculus,

50 Mus leucopus,

51 'Arvicola riparius,

52 Arvicola rufescens,

53 Arvicola hirsutus,

54 Arvicola oneida,
Brown Rat (introduced. m. \& f.). Cabinet. Black Rat (introduced).

American Black Rat. - - Cabinet.

Common Mouse (m. \& f.). - Cabinet. Jumping Mouse (f.). - - - Cabinet. Marsh Meadow Mouse.

Tawny Meadow Mouse. - - Cabinet. Beaver Field Mouse. - - Cabinet. Oneida Meadow Mouse.

55 Arvicola albo-rufescens, Light-colored Meadow Mouse. 56 Arvicola xanthognathus, Yellow-cheeked Meadow Mouse.

\section{FAMILY LEPORIDAs.}

57 Lepus nanus, 58 LePUS AMERICANUS,
American Gray Rabbit (two). Cabinet. Northern Hare. - - - - Cabinet.

\section{ORDER V. EDENTATA.}

\section{ORDER VI. UNGULATA. •}

FAMILY ELEPHANTIDA.

59 Elephas primigenius,

60 Elephas americanus,

61 Mastodon maximus,
Fossi? Elephant.

American Elephant (fossil).

Great Mastodon (fossil). 
F.AMILY SUID AE.
Latin Names.
English Names.

62 Sus scrofa, var. domesticus, Common Hog (introduced).

FAMILY EQUIDA.

63 EquUts caballus,

64 Equus asinus, Horse (introduced).

65 EQUUS MAJOR, Ass (introduced).

Fossil Horse.

FAMILY BOVID A.

66 Bos taurus,

Common $O x$ (introduced).

\section{FAMILY CAPRID $Æ$.}

67 Ovis ARIEs,

Domestic Sheep (introduced).

FAMILY CERVID无.

68 Cervus virginianus,

96 Cervus alces,

70 Elaphus canadensis,

71 Elaphus americanus,

72 Rangifer tarandus,
American Deer. - - - - Cabinet. Moose. - - - - - - Cabinet. American Stag, or Elk (horns). Cabinet. Fossil Stag.

Reindeer (extirpated?).

\section{ORDER VII. CETACEA.}

\section{FAMILY MANATID $\nRightarrow$.}

FAMILY BAL ÆNID\#.

73 Balma mysticetus, Right Whale.

74 Physeter macrocephalus, Sperm Whale.

75 Rokqualus rostratus, Beaked Rorqual.

76 RoRquaLUS boREaLIS, Northern Rorqual.

\section{FAMILY DELPHINID开.}

77 Globicephalus melas,

78 Phoc mna communis,

79 Phocma orca,

80 Delphinus Delphis,
Social Whale.

Common Porpoise.

Grampus.

Sea Porpoise.

Castoroides ohroensis, Fossil Beaver (cast of skull \& lower jaw)." Cabinet.

* DE KAY, in his Zoology of New-York, describes the Fossil Beaver as extralimital. The original specimen from which the casts in the collection were taken was fornd near Clyde in Wayne county, N. York. We therefore place the Fossil Beaver in the catalogue of the New-York Mammalia. 


\title{
Z00LOGY OF NEW-YORK,
}

OR THE

\section{$\mathbb{N} \mathfrak{e} \mathfrak{w} \sim \mathbb{1} \mathfrak{o} \mathfrak{x} \mathfrak{k} \quad \mathfrak{f} \mathfrak{a} \mathfrak{u} \mathfrak{n} \dot{\mathfrak{a}}$}

\author{
BY JAMIES E. DE KAY.
}

PART II. BIRDS. 



\section{Z00 L 0 GY.}

\section{CLASS II. BIRDS.}

\section{ORDER I. ACCIPITRES.}

FAMILY VULTURIDA.

Latin Name.

1 Cathartes aura,
English Name.

Turkey Buzzard. . - - -

In the

\section{FAMILY FALCONIDA.}

2 Aquila chrysaetos,

Golden Eagle. - - - Cabinet.

3 Haliaetos leucocephalus, Brown or Bald Eagle (m. \& 2 f.).

Cabinet.

4 Pandion carolinensis, American Fish Hawk. - - Cabinet.

5 Buteo sancti-rohannis, Rough-legged Buzzard(m. \& f.). Cabinet.

6 Buteo borealis, Red-tailed Buzzard (m.\& f.). Cabinet.

7 Buteo hyemalis, Red-shouldered Buzzard. - Cabinet.

8 Buteo pennsylvanicus, Broad-winged Buzzard (m.\& f.). Cabinet.

9 Nauclerus furcatus,

10 Falco anatum,

Swallow-tailed Hawk. - - -

11 Falco colombarius,

Duck Hawk (m.\& f.). - - Cabinet. Pigeon Hawk. - - - Cabinet.

12 Falco sparverius,

13 Astur fuscus,

14 Astur cooperi,

15 Astur atricapillus, 16 Circus uliginosus, American Sparrowhawk (m.\& f.). Cabinet. Slate-colored Hawk (m. \& f.). Cabinet. Cooper's Hawk. - - - Cabinet. American Goshawk (m.\& f.). Cabinet. Marsh Harrier, - . . Cabinet. 
FAMILY STRIGIDZE.

Latin Names.

17 Surnia Funerea,

18 SURNIA NYCTEA,

19 Bubo virginianus,

20 Bubo asio,

21 Syrnium cinereum,

22 Otus americanus,

23 Otus palustris,

24 Ulula nebulosa,

25 Ulula acadica,

26 Strix pratincola,
English Names.

Hawk Orol. - - - - Cabinet.

Snowy Owl (m. \& f.). - - Cabinet.

Great Horned Owl (m. \& f.). Cabinet.

Little Screechoul (m. f. \& y.). Cabinet. Great Grey Owl (m.\& f.). - Cabinet. Long-Eared Owl (m.\& f.). - Cabinet. Short-eared Owl (m.\& f.). - Cabinet. Barred Owl (2 m.). - - - Cabinet. Acadian Oul (m. \& f ). - - Cabinet. American Barn Owl. - - Cabinet.

\section{ORDER II. PASSERES.}

FAMILY CAPRIMULGIDA.

27 Caprinulgus vociferus, Whippoorwill. - - - Cabinet, 28 Chordeiles americanus, Nighthawk. - - - Cabinet.

\section{FAMILY HIRUNDINID正.}

29 Chemtura pelasgia,

30 Hirundo furpurea,

31 Hirundo bicolor,

32 Hirundo RIfaria,

33 Hirundo rufa,

34 Hirundo fulva,
Chimney Swallow (m. \& f.), Cabinet, Purple Martin (m. \& f.). - Cabinet. White-bellied Svallow. - - Cabinet. Bank Swallow. - - - Cabinet. Barn Swallow. - - - Cabinet. Cliff Swallow (m. \& f.). - - Cabinet.

FAMILY AMPELID正.

35 Bonbrctlda garrula, Black-tlloated Waxwing. - Cabinet. 36 Bombycilda carolinensis, Cedarbird (m. \& f.). - - - Cabinet.

\section{FAMILY ALCEDINID压.}

37 AlCEDo ALCYON, Belted Kingfisher (two). - Cabinet. FAMILY TROCHILID瓜.

38 Trochilus colubris, Red-throated Huinmingbivd (m. \& f.). Cabinet. 


\section{FAMILY CERTHID}

Latin Names.

39 Sittia carolinensis,

40 Sitta canadensis,

41 Certhia americana,

42 Mniotilita varia,

43 Troglodytes xedon,

44 Troglodytes americanus, Wood Wren.

45 Troglodytes ludovicianus, Mocking Wren (m. \& f.). - Cabinet. 46 Troglodytes palustris, Marsh Wren (m. \& f.). - - Cabinet. 47 Troglodytes hyemalis, Winter Wren (m. \& f.). - - Cabinet. 48 Troglodytes erevirostris, Short-billed Wren. - - Cabinet.

FAMILY PARID死.

49 Parus bicolor,

50 Parus atricapillus,

51 Parus oarolinensts, Crested Tit. - " - - Cabinet.

\section{English Names.}

White-breasted Nuthatch. - Cabinet. Red-bellied Nuthatch (m.\& f.). Cabinet. Brovon Creeper (f.). - - - Cabinet. Varied Creeping Warbler (m.\& f.). Cabinet. House Wren (three). - - "- Cabinet. Black-cap Tit. - - - Cabinet. Carolina Tit. - - - - Cabinet.

\section{FAMILY SYLVIADA.}

52 Regulus satrapa,

53 Regulus caleENDUla,

54 Sialia wilsoni,

Golden-crested Kinglet. - - Cabinet. Ruby-crowned Kinglet. - - Cabinet. Bluebird (m. \& f.). - - Cabinet.

FAMILY MERULIDA.

55 Orpheus polygloteds,

56 OrpheUs rufus,

57 Orphets carolinensis,

58 Merdia migratoria,

59 Merula mustelina,

60 Merula solitaria,

61 Merula olivacea,

62 Merula wilsoni,

Common Mockingbird. - - Cabinet. Brown Thrush (m. \& f.). - Cahinet. Catbird (m. \& f.). - - - Cabinet. American Robin (m. \& f. and an albino). Cabinet. Wood Thrush (m. \& f.). - - Cabinet. Hermit Thrush. - - - Cabinet. Olive-backed Thrush. - - Cabinet. Wilson's Thrush (f.). - - Cabinet.

\section{FAMILY MOTACILLID $\approx$.}

63 Anthus ludovicianus, American Titlark (f.). - - Cabinet.

64 Seiurus noveboracensts, New-York Water Thrush (m. \& f.).

Cabinet.

65 Seiurus aUrocapillus, Ovenbird. Cabinet. 


\section{FAMILY SYLVICOLID $\mathrm{E}$.}

Latin Names.

66 Trichas marilandica,

67 Trichas philadelphia,

68 Vermivora pennsylvanica, Worm-eating Warbler.

- Cabinet.

69 VERMIVORA SWAinsoni,

70 Vermivora solitaria,

71 Vermivora chrysoptera,

72 Vermivora peregrina,

73 Vermivora rubricapilla, Nashville Warbler. - - - Cabinet.

74 Vermivora celata,

75 Sylvicola coronata,

76 Sylvicola nuficapilla,

77 Sylvicola maculosa,

78 Sxlvicola pardalina,

79 Sxlticola cerrulea,

80 Sylvicola bLackbURNia,

81 Sylvicola castanea,

82 Sylvicola striata,

83 Sylvicola discolor,

84 Sylvicola' aMericana;

85 Sylvicola canadensis,

86 Sylvicola mstiva,
Orange-crowned Warbler (m.\& f.). Cabinet. Myrtlebird. - . - - Cabinet. Reḋpoll Warbler (m. \& f.). - Cabinet. Spotted Warbler. - - - Cabinet. Spotted Canada Warbler (m.\& f.). Cabinet. Blue-grey Warbler. - - Cabinet. Blackburnian Warbler. - -

Bay-breasted Warbler (f.). - Cabinet. Blackpoll Warbler. - - - Cabinet. Prairie Wurbler. - - - Cabinet. Blue Yellow-backed Warbler (f.). Cabinet. Black-throated Blue Warbler, Cabinet. Summer Yellowbird (m. \& f.). Cabinet. 87 Sylvicola viRens, Black-throated Green Warbler (m. \& f.). Cabinet. 88 Sylvicola pinus, Pine Warbler (m. \& f.). - Cabinet. 89 Sylvicola icterocephala, 90 Syliticola farus,

91 Sylvicola maritima,

92 Syluicola formosa,

93 Wilsonia mitrata, Chesnut-sided Warbler. - - Cabinet. Hemlock Warbler. . . - Cape-May Warbler. - - - Cabinet. Kentucky Warbler. - - - Cabinet. Hooded Warbler (m. \& f.). - Cabinet. 94 Wilsonia pusilla, Green Black-capped Warbler (m.\& f.). Cabinet. 


\section{FAMILY MUSCICAPID $\approx$.}

Latin Names.

95 Culicivora c 死rulea,

96 Muscicapa ruticilla,

97 Muscicapa acadica,

98 Muscicapa flaviventris,

99 Muscicapa virens,

100 Muscicapa fusca,

101 Tyrannus intrepidus,

102 Tyrannus coOperi,

103 Trrannus crinitus,
English Names.

Blue-grey Gnatcatcher. - - Cabinet.

American Redstart (m. \& f.). Cabinet.

Small Green-crested Flycatcher (f.). Cabinet.

Yellow-bellied Flycatcher.

Wood Pewee (f.). - - - - Cabinet.

Phabebird. - - - - - Cabinet.

Kingbird (m. \& f.). - - - Cabinet.

Olive-sided Kingbird (f.). - Cabinet.

Great-crested Kingbird. - Cabinet.

FAMILY VIREONIDE.

104 Vireo FLAVIFRONS,

105 Vireo solitarius,

106 VIREO NOVEBORACENSIS,

107 Vireo gilvus,

108 Vireo olivaceus,

109 ICTERIA VIRIDIS,
Yellow-throated Greenlet (three). Cabinet. Solitary Greenlet. - - - Cabinet. White-eyed Greenlet (m. \& f.). Cabinet. Warbling Greenlet. - - - Cabinet. Red-eyed Greenlet (m. \& f.). Cabinet. Yellow-breasted Chat (m.\& f:). Cabinet.

FAMILY LANID E.

110 Lanius septentrionalis, Northern Buicherbird (m. \& f.). Cabinet. FAMILY CORVID无.

111 GarRULUS CRISTATUS,

112 Garrulus canadensis,

113 Pica caudata,

114 Corvus americanus,

115 Corvus corax,

116 Corvus ossifragus,
Blue Jay (m. \& f.). - - - Cabinet. Canada Jay. Magpie. - - - - - Cabinet.

Common Crow (m. \& f.). - Cabinet. Raver. - - - - - - - Cabinet. Fish Crow. - - - - - Cabinet.

FAMILY QUISCALID

117 Quiscalus versicolor, Common Crow Blackbird (m. \& f.). Cabinet. 118 Quiscalus Ferrugineus, Rusty Crow Blackbird (m. \& f.). Cabinet, 119 Sturnella ludovicianus, Meadoro Lark (m. \& f.). - Cabinet.

120 ICterus BaLTIMORE,

121 ICTERUS sPurios,
Golden Oriole (m. \& f.)). - Cabinet.

Orchard Oriole (m. \& f.). - Cabinet. 
Latin Names.

122 I

123 Molothros pecoris,

124 Dolichonyx oRyzivorus,
English Names.

Red-winged, Oriole (m. \& f.). Cabinet.

Cow Bunting (m. \& f.). - - Cabinet. Boblink (m.\& f.). - - - Cabinet.

\section{FAMILY FRINGILLID $Æ$.}

125 CócCoborus CERTLEUS,

Blue Grosbeak.

126 Coccoborus ludovicianus, Rose-breasted Grosbeak (m. \& f.). Cabinet.

127 Struthus hyemalis,

128 Fringilla iliaca,

129 Fringilla melodia,

130 Fringilita graminea,

131 Fringilla pennsylvanica,

132 Fringilda leucophr.ys,

133 Emberiza americana,

134 EMrberiza Passerina,

135 EMberiza henslowi,

136 Eimberiza pustlla,

137 Emberiza socialis,

138 Emberiza canadensis,

139 Emberiza sayana,

140 EnIBERIZA LINCOLNI,

141 Ammodramus maritimus,

142 Ammodramus caddacutus,

143 Amimodramus palustris,

144 Carduelis tristis,

145 Carduelis pinus,

146 Linaria minor,

147 Linaria borealis,

148 Erythrospiza purpurea,

149 Pitylus cardinalis,

150 Pipilo erythrophthalmus, Chewink, or Ground Robin (two). Cab.

151 Spiza cyanea,

152 Pyranga estiva,
Snowbird. - - - - - Cabinet.

Fox-colored Sparrow (m. \& f.). Cabinet. Soug Sparrow. - - - Cabinet.

Bay-winged Sparrow (m. \& f.). Cabinet.

White-throated Sparrow (m. \& f.). Cab.

White-crouned Sparrow. - Cabinot.

Black-throated Bunting (m. \& f.). Cab.

Yellow-vinged Bunting (m.\& f.). Cab.

Varied Bunting. - - - - Cabinet.

Field Bunting (m. \& f.). - Cabinet.

Chipbird. - - - - Cabinet.

Tree Bunting (m. \& f.). - Cabinet.

Savarnah Bunting (m.\& f.). Cabinet.

Blue-striped Bunting.

Seaside Finch. - - - - Cabinet.

Quailhead (m.\& f.). - - - Cabinet.

Swamp Finch (m.\& f.). - - Cabinet.

Yellowbird, or American Goldfinch

(three). Cabinet.

Pine Finch (m. \& f.). - - Cabinet:

Lesser Redpoll (m. \& f.). - Cabinet.

Mealy Redpoll.

Crested Purple Finch (f.). * Cabinet. Cardinal Grosbeak (m. \& f.). Cabinct. Indigobird (m.\& f.). - Cabinet. Redbird. - . - - Cabinet. 
Latin Names.

153 Prranga rubra,
English Names.

Black-winged Redbird (m. \& f.). Cabinet.
155 Plectrophanes nivalis,

156 Aladda cornuta,

157 Corythus enucleator,

158 Loxta americana,

159 LOXIA LEUCOPTERA,
White Snowbird. - - - Cabinet.

Horned Lark (m. \& f.). - - Cabinet.

Pine Bulfinch (three). - - Cabinet.

American Crossbill (m: \& f.). Cabinet. White-winged Crossbill (m. \& f.). Cabinet.

\section{FAMILY PICIDE.}

160 Picus pileatus,

Crested Woodpecker (m.\& f.). Cabinet.

161 Prcus erythrocephadus, Red-headed Woodpecker (m. \& f.). Cabinet.

162 Picus vizlosus,

163 Picus pubescens,

164 Picus varius,

165 Picus carolinus,

166 Picus arcticus,

167 Picus hirsutus,
Hairy Woodpecker. - - - Cabinet.

Downy Woodpecker. - - Cabinet.

Yellow-bellied Woodpecker (m. \& f.). Cabinet.

Red-bellied Woodpecker. - Cabinet. Arctic Woodpecker. - - - Cabinet. Banded Woodpecker.

168 Picus adratus, Golden-winged Woodpecker, or Clape (two). Cabinet.

\section{FAMILY CUCULID平.}

169 Coccyzus americanus,

Yellow-bellied Cuckoo. - - Cabinet.

170 Coccyzus erythrophthalmus, Black-billed Cuckoo (m. \& f.). Cab.

FAMILY COLUMBID死.

171 Ectopistes migratoria, Wild Pigeon (m.\& f.). - - Cabinet.

172 Ectopistes CAROLINENSIS,

Carolina Turtle Dove.

\section{ORDER III. GALLIN正.}

\section{FAMILY PHASIANID瓜.}

173 Meleagris gallopavo, Wild Turkey. - - - - Cabinet.

\section{FAMILY TETRAONID尼.}

174 Ortyx virginiana,

175 Tetrao unbellus,

176 Tetrao cupido,

177 Tetrao canadensis,
Quail (m. \& f., and a circle of five). Cabinet. Ruffed Grouse (m. f. \& у.). - Cabinet. Pinnated Grouse (m. \& f.). - Cabinet. Spotted Grouse (m.\& f.). - Cabinet. 


\section{ORDER IV. GRALLस.}

\section{FAMILY CHARADRIDæ.}

Latin Names.

English Names.

178 Charadrius semipalmatus, American Ring Plover (m. \& f.). Cab.

179 Charadrius melodus, Piping Plover. - - - Cabinet.

180 Charadrius wilsonius, Wilson's Plover. - - - - Cabinet.

181 Charadrius voctferus, Kildeer Plover (m.\& f.). - Cabinet.

182 Charadriug virginiacus,

183 Squatarola helovetica,

184 Strepsilas INTERPRES,

185 Hzmatopus palifatus,

Golden Plover (m. \& f.). - Cabinet.

Whistling Plover. - - Cabinet.

Turnstone (m. \& f.). - - - Cabinet.

American Oystercatcher (m.\& f.). Cab.

FAMILY GRUID $Æ$.

186 Grus americana,

187 ARdea herodias,

188 Ardea leEdeE,

189 Ardea CANDIDISSIMA,

190 ARDEA CERULEA,

191 Ardea lddoviciana,

192 ARDEA VIRESCENS,

193 ARdea exJlis,

194 ARDEA MINOR,

195 ARDEA DISCORS,

196 Andea violacea,
American Crane.

Great Blue Heron (m. \& f.). Cabinet. Great White Heron.

White-crested Heron (m. \& f.). Cabinet. Blue Heron (m. \& f.). - - Cabinet. Louisiana Heron.

Green Heron. - - - - - Cabinet.

Small Bittern. - - - Cabinet. American Bittern. - - - Cabinet. Black:crowned Night Heron (m. \& f.). Cabinet. Yellow-crowned Night Heron (m. \& f.). Cabinet.

197 Ibis alba, FAMILY TANTALIDE.

198 Ibis MeXicants, White Ibis.

Glossy Ibis (m.\& f.). - - Cabinet.

FAMILY SCOLOPACID瓜.

199 Numenids longirostris, Long-billed Curlew. - - Cabinet.

200 Nunenius hudsontcus, Jack Curlew (m.\& f.). - - Cabinet. 201 Numenids borealis, Small Esquimaux Curlew. - Cabinet. 202 Hemipalma himantopus, Long-legged Sandpiper. - Cabinet. 203 Heteropoda semtpalmata, Semipalmated Sandpiper. - Cabinet. 
Latin Names.

204 Tringa maritima,

205 Tringa rufescens,

206 Tringa subarquata,

207 Tringa cinclú,

208 Tringa schinzi,

209 Tringa pectoralis,

210 Tringa canutus,

211 Tringa pusilla,

212 Caledris arenaria,

213 Totanus mactlarius,

214 Totanus bartramitus,

215 Totanus flavipes,

216 Totanus chloropygius,

217 Totanus melanoleucus,

218 Totanus semipalmatus,

219 Limosa fedoa,

220 Limosa hudsonica,

221 Scolopax noveboracensis,

222 Scolopax wilson,

.223 Rusticola minor,
English Names.

Purple Sandpiper (f.). - - Cabinet.

Buff-breasted Sandpiper. - Cabinet.

Curlew Sandpiper (m. \& f.). Cabinet.

Black-breasted Sandpiper. -

Schinz's Sandpiper (m. \& f.). Cabinet

Pectoral Sandpiper. - - Cabinet.

Red-breasted Sandpiper (m. \& f.). Cab.

Wilson's Sandpiper (m. \& f.). Cabinet.

Sanderling (m. \& f.). - - Cabinet.

Spotted Sandlark (m. \& f.). Cabinet.

Grey Plover. - - - - Cabinet.

Yellowlegs. - . - - Cabinet.

Solitary Tatler (f.). - - - Cabinet.

Varied Tatler (m.\& f.). - - Cabinet.

Willet. - - - - - Cabinet.

Marlin (m.\& f.). - - - - Cabinet.

Ring-tailed Marlin (m.\& f.). Cabinet.

FAMILY RALLIDÆ.

224 Rallus crepitants,

225 Rallus elegans,

226 Ralius virginianus,

227 Ortygometra carolina,

228 Ortygometra Noveboracensis, New-York Rail. - - Cabínet.

229 Gallinula galeata,
Common American Snipe (m. \& f.). Cabinet. American Wondcock. - - Cabinet.

\section{FAMILY RECURVIROSTRID A.}

230 Hrmantopts nigricoldis, Lawyer. - - - - Cabinet

231 Recurvirostra americana, American Avoset. - - - Cabinet. 
FAMILY PHALAROPODID瓜.

Latin Names.

English Names.

232 Phalaropus fulicaries,

Red Phalarope.

233 LOBIPES HYPERBOREUS,

Hyperborean Lobefcot (m. \& f.). Cabinet,

234 Holopodius widsoni,

Wilson's Holopode.

\section{ORDER V. LOBIPEDES.}

FAMILY PODICIPID在,

235 Fulica americana,

236 Poniceps cornutus,

237 Podiceps cristatus,

238 Podiceps RUBRicollis,

239 Hydroka carolinensis,
American Coot (m. \& f.). " Cabinet, Horned Grebe. - - - - Cabinet.

Crested Grebę. - . - ' - Cabinet.

Red-necked Grebe. - - -

Dipper (three), - - - Cabinet.

\section{ORDER VI. NATATORES.}

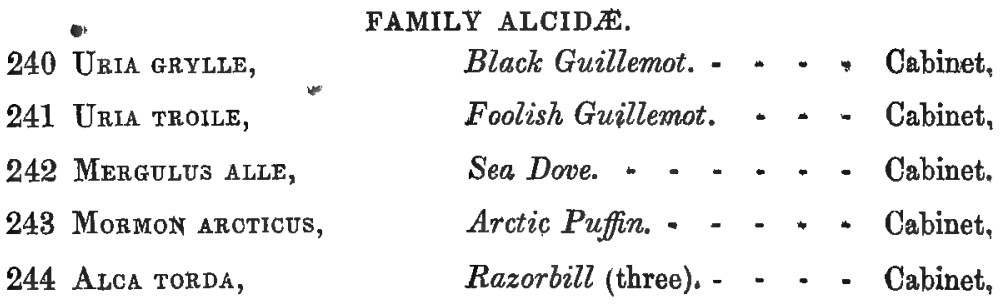

FAMILY COLYMBIDAE.

245 Colymbus glacialis, Great Loon, or Diver. - - Cabinet,

246 Colymbos septentrionalis, Red-throated Loon (m. \& f.). Cabinet.

FAMILY PROCELLARID.AE.

247 Puffinds cinereus,

248 Puffinds obscords,

249 Thathassidroma wilsoni,

250 Thalassidroma LeachI,
Large Shearwater. - - Cabinet, Little Shearwater. - - - Cabinet. Wilson's Petrel. - - - - Cabinet. Fork-tailed Petrel. - - - Cabinet,

FAMILY PELECANIDE.

251 Phalacracorax carbo,

Cormorant.

252 Phalacracorax dilophus, Double-crested Cormorant (two). Cab.

253 Pelecanus fuscus, Brown Pelican. - - - Cabinet, 254 Sula americana, American Gannet. - - - Cabinet. 
BIRDS.

\section{FAMILY LARIDEE.}

Iatin Names.

255 RHYNCHOPS NIGRA;

256 Sterna hirundo,

257 Sterna cayana,

258 Sterna NIGRA,

259 SterNa ANGLICA,

260 Sterna arctica,

261 Sterna cantiaga,

262 Sterna dougalla,

263 STERNA ARGENTEA,

264 Larus argentatus,

265 Larus marinus,

266 LARUS ZONORRHYNCHUS,

267 LARUS ATRICILLA,

268 I.ARUS boNaparti,

269 LARUS sabiNi,

270 I AaRUs TRIDACtYLUs,

271 LEsTRIS BUFFoNI,

272 LestRIs RICHARDSONII,

273 Lestris pomarinus,
English Names.

Black Skimmer (m. \& f.). - Cabinet.

Common Tern. - - - - Cabinet.

Cayenne Tern. - - - - Cabinet.

Black Tern (m. \& f.). - - Cabinet.

Marsh Tern (m. \& f.). - - - Cabinet.

Arctic Tern.

Sandwich Tern.

Roseate Tern.

Silvery Tern (m. \& f.). . - - Cabinet.

Winter Gull (1 \& y.) - - Cabinet.

Great Black-backed Gull. - Cabinet.

Common American Gull (2 old

and 3 young). Cabinet.

Laughing Gull (three), - - Cabinet.

Bonaparte's Gull (m.\& f). Cabinet.

Fork-tailed Gull.

Kittiwake, or Threentoed Gull.

Arctic Hawk Gull. -

Richardson's Hawk Guli (three). Cabinet.

Pomarine Hawk Gull (three). Cabinet.

- FAMILY ANATIDA.

274 Mergus Merganser, Buff-breasted Sheldrake (m. \& f.). Cabinet. 275 Mergus serrator, 276 Mirgus cucullatus, Red-breasted Sheldrake (m. \& f.). Cabinet. 277 Fuligula valisneria, Hooded Sheldrake (m. \& f.). Cabinet. Canvasback (m. \& f.). - - Cabinet. 278 Fuligula erythrocephala, Redhead. - - - - - Cabinet. 279 Fuligula marila, Broadbill. - - - - - Cabinet. 280 Fuligula MiNor, Creek Broadbill. - - - Cabinet.

281 Fuligula rofitorques, Bastard Broadbill. - - - Cabinet. 282 Fuligdla labradora, Pied Duck (f.). - - - - Cabinet. 283 Fuligula RJibida,

Ruddy Duck (m. \& f.). - - Cabinet. 
Latip Names.

284 FULIGUira GEACIALIS,

285 FULigula A hibeola,

286 FULiguba clangula,

287 FuligULA HISTRIONICA,

288 Fuligula Mollissima,

289 FULIGULA SPECTABILIS,

290 Fuligula perspicill.ata,

291 Fuliguld AMERICANA,

292 Fuligula FuscA,

293 Anas sponsa,

294 Anas Discors,

295 ANas carolinensis,

296 Anas acUta,

297 ANas CLYPEATA,

298 ANAs STREPERA,

299 ANas OBSCURA,

300 Anas americana,

301 ANAs RENElope,

302 Anas boschas,

303 Anser canadensis,

304 ANser albifrons,

305 ANser hyperboreus,

306 ANEER BERNICLA,

307 ANsER HUTCHINSI,

308 CygNus americaNus,
English Names.

Oldwife, or Squaw Duck (four). Cabinet. Buffe-headed Duck. - * - Cabinet. Whistler. - - - - - Cabinet.

Harlequin Duck. - - - - Cabinet.

Eider Duck (m. \& f.). - - Cabinet.

King Duck. - - - - Cabinet.

Surf Duck or Coot. - - - Cabinet.

Broad-billed Coot. - - Cabinet.

White-winged Coot. - . Cabinet.

Wood Duck (m. \& f.). . Cabinet.

Blue-winged Teal (m.\& f.). Cabinet.

Green-winged Teal (three). - Cabinet.

Pintail Duck (m. \& f.). - - Cabinet. Shoveller, or Spoonbill (m. \& f.). Cabinet.

Grey Duck, or Gadwall. - Cabinet. Black Duck. - . - . - Cabinet. American Widgeon, or Baldpate. Cab.

European Widgeon. "

Mallard (m. \& f.). - - - Cabinet. Wild Goose. - - * - Gabinet.

White-fronted Goose. - . _ _-

Snow Goose. :- - - - Cabinet. Brant. - - - - - - Cabinet. Hutchins's Goose.

American Swan. - . - Cabinet.

Purple Gallinule*.

Cabinet.

309 Gallindla martinica,

- This specimen was obtained by Mr. J. G. Bes, of Now-York, within this State,
and furnished by him to the Collection. It is considered extraalimital by Dr. Dit KAy (See his Birds of New-York, p, 264), . 


\title{
Z00LOGY OF NEW-YORK,
}

\author{
OR THE
}

$\mathbb{N} \mathfrak{e} \mathfrak{w}-\mathfrak{p} \cdot \mathfrak{o} \mathfrak{r} \mathfrak{i} \quad \mathfrak{f} \mathfrak{a} \mathfrak{u} \mathfrak{n} \mathfrak{a}$

PARTS III \& IV, REPTILES AND AMPHIBIA. 



\section{Z $00 \mathrm{~L} 0 \mathrm{GY}$.}

\section{CLASS III. REPTILES,}

\section{ORDER I. TESTUDINATA,}

FAMILY CHELONID $A$.

Latin Name.

1 Chalonia mydas,

2 Sphargis coriacea,

3 Trionyx ferox,

4 Chelonura serpentina,

5 Emys paldustris,

6 Emys tegrapin,

7 Emys picta,

8 Eims guttata,

9 Emys insculpta,

10 Emys rubriventriș,

11 Eirys muHLENbẹRgir,

12 Emys GeograpHica,

13 Emys pseudogeographica,
English Name. Green Turtle.

Leather Turtle,

Soft-shelled Turtle.

Snapping Turtle (m. f. \& eggs). Cabinet.

Saltuater Terrapin.

Smooth Terrapin (2 shells). - Cabinet.

Painted Tortoise (m. d f.). - Cabinet.

Spotted Tortoise (m. f. f.). Cabinet.

Wood Terrapin (m.\& f.). - Cabinet.

Red-bellied Terrapin.

Muhlenberg's Tortoise.

Geographic Tortoise,

Pseudogeographic Tortoise.

14 Kinostemon pennsyevantoum, Mud Tortoise.

15 Sternotherds odoratus, Musk Tortoise (in alcohol). - Cabinet.

16 Cistuda carolina, Common Box Tortoise (m. \& f.). Cabinet.

17 Cistuda blandingit, Blanding's Bax Tortoise. 


\section{ORDER II. SAURIA.}

FAMILY SCINCID无,

Latin Names. English Names.

18 Scincus fasciatus, Blue-tailed Skink.

FAMILY AGAMIDA.

19 Tropidolepis unduhatus, Brown Swift (in alcohol). - Cabinet.

\section{ORDER III. OPHIDIA.}

\section{FAMILY COLUBERIDA.}

20 Colubfr constrictor, Black Sqake (2 in alcohol). - Cabinet,

21 Coldber aldeghaniensis, Pilot Blackisnake (in alcohol). Cabinet.

22 Colubeq getulus,

Chain Snake (in alcohol). - Cabinet.

23 Coluber eximius,

Milk Snake (4 in alcohol). - Cabinet.

24 Coluber punctatus,

Ring Srake (in alcohol). - - Cabinet.

25 Coluber vernalis, Grass Snake (one, and 1 in alcohol). Cabinet.

26 Tropidonotus sipedon, Water Snake (in alcohol). - Cabinet.

27 Tropidonotus temia, Striped Snake (one, and 1 in alc.). Cab.

28 Tropidonotus leberis, Yellow-bellied Snake (in alcohol). Cahinet.

29 Tropidonotus dekayi, Small Brown Snake (one, \& 1 in alc.). Cab.

30 Leptophis SaUdita, Ribbon Snake (one, \& 1 in ąle,). Cabinet.

31 Calamaria amgena, Red Snake.

32 Heterodon Platyrrhinos, Hog nosed Srake. - - ' Cabinet.

FAMILY CROTALID A世.

33 Trigonocephalus contortrix, Copperhead.

34 Crotalus durissus,

Northern Rattlesnake (m. \& f., and 2 in alcohol and 4 young). Cabinet.

35 Crotalophorus teraminus (Say). Triple-spotted Rattlesnake. Cab. (See Annual Report of 1853.) 


\section{Z 00 L 0 G Y.}

\section{CLASS IV. AMPHIBIA.}

FAMILY RANID无.

Latin Name.

1 RaNa PIPIENS,

2 RaNa HoRICONENSIS,

3 Rana fontinalis,

4 Rana palustris,

5 RaNa halecina,

6 Rana syivatica,

7 Scaphiopts solitarius,

8 Bufo americanus,

9 HyLodes PICKERINGI,

10 Hylodes gryllus,

11 Hyia versicolor, Northern Tree-toad (one, and 2 in alc.). Cabinet.

12 HyLa squirella,
English Name. Bulfrog (2 in alcohol). - - Cabinet. Large Northern Bulfrog (two, and 2 in alcohol). Cabinet. Spring Frog (in alcohol). - Cabinet. Marsh Frog (in alcohol). - - Cabinet. Shad Frog (two, and 1 in alc.). Cabinet. Wood Frog (one, and 2 in alc.). Cabinet. Hermit Spadefoot.

Common American Toad (one, and 2 in alcohol). Cabinet.

Pickering's Hylodes.

Cricket Hylodes.
Squirrel Tree-toad (two). - Cabinet.

\section{FAMILY SALAMANDRID开.}

13 Salamandra symmetrica, Yellow-bellied Salamander (m. \& f.). Cab. 14 Salantandea subviolacea, Violet-colored Salamander (one, and 1 in alcohol). Cabinet.

15 Salamandra erythronota, Red-backed Salamander (m. \& f.). Cab.

16 Salamandra picta,

17 Salamandra SALMONEA,
Painted Salamander (in alcohol). Cab, Salmon-colored Salamander. 
Latin Names.

18 SALAMANDRA Fasciata,

19 Sajamandra longicatuda,

20 Salamandra grandhata,

21 Salamandra bilineata,

22 Salamandra RUBra,

23 Salamandra coccinea,

24 Salamandra glutinosa,

25 TRITON TIgRINUS,

26 Triton Miliepunctatus,

27 Triton Niger,

28 TRITON PORPHYRITICUS,
English Names.

Blotched Salamander.

Long-tailed Salamander.

Granulated Salamander.

Striped-backed Salamander.

Red Salamander (m.\& f.). - Cabinet. Scarlet Salamander.

Blue-spotted Salamander

Tiger Triton.

Crimson-spotted Triton (m. \& f.), Cab.

Dusky Triton.

Grey-spotted Triton (m.\& f.). Cabinet,

FAMILY SIRENID A.

29 Menobranchus lateralis, Banded Proteus.

FAMILY AMPHIUMID $\approx$.

30 Menopoma alleghaniensis, Allegany Hellbender.

31 Plethodon oinereds (Baird). - - - - - - Cabinet.

32 HEMidadTýd sodtatoM (Baird). - - - - - Cabinet. 


\section{Z00LOGY OF NEW-YORK,}

OR THE

$\mathbb{N} \mathfrak{e} \mathfrak{w}-\mathfrak{W} \mathfrak{o} \mathfrak{x} \mathfrak{k} \quad \mathfrak{F} \mathfrak{a} \mathfrak{a} \mathfrak{n} \mathfrak{a}$

BY JAMES E. DE KAY.

PART V. FISHES。 



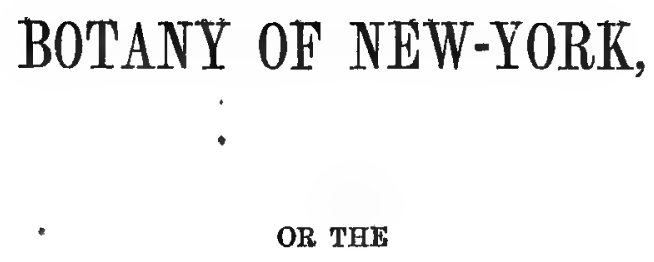

$\mathbb{N} \mathfrak{e} \mathfrak{w} \sim \mathbb{1} \mathfrak{o} \mathfrak{x} \mathfrak{i} \quad \mathfrak{f} \mathfrak{l} \mathfrak{o} \mathfrak{r}$ BY JOHN TORREY.

DIVISION I. PHANOGAMOUS PLANTS. 



\section{B 0 T A N.}

\section{DIVISION I. PHANOGAMOUS PLANTS.}

\section{CLASS I. EXOGENOUS PLANTS.}

Vol. 1.]

ORDER I. RANUNCULACEE.

Latin Name.

English Name.

In the

1 Chematis ochroleuca, Silky Virgin's-bower (3 specimens). Cabinet.

2 Clematis virginiana,

Virginian Virgin's-bower(3). Cabinet.

3 Clematis verticillaris, Whorl-leaved Virgin's-bower(3). Cabinet.

4 ANemione nemorosa,

Wood Anemone(6). - - - Cabinet.

5 ANeMoNe crlindrica, Cylindrical-headed Windflower(4). Cabinet.

6 Anemone virgintana,

Thimbleweed(3). - - Cabinet.

7 Anemone MULTifida,

Cut-leaved Windflower.

8 Anemone penNSYlvanica,

Pennsylvanian Windflower(2). Cabinet.

9 Hepatica triloba,

Common Liverleaf(6). - - Cabinet.

10 Ranunculus aquatilis,

11 Ranunculus flammula,

12 Ranunculus reptans,

13 Ranunculus pusilizus,

14 Ranunculdos cymbalaria,

15 Ranunculus abortivus,

16 Ranunculus scelleratus,

17 RanUNCULUS PURSHI,

18 Ranunculus acris, Water Crowfoot(3). - - Cabinet. Spearwort(2). - - - Cabinet. Least Spearwort(7). - - Cabinet. . Small-flowered Crowfoot(3). Cabinet. Sea Crowfoot(5). - - - Cabinet. Kidney-leaved Crowfoot(2). Cabinet. Celery-leaved Cronfoot(2). Cabinet. Pursh's Cronfoot(2). - - Cabinet. Tall Crowfoot(2). - - Cabinet. . 
NEW·YORK STATE COLLECTION.

Latin Names.

English Names.

19 Ranunculus repens, Creeping Crowfoot(5). - Cabinet.

20 Ranunculus fascicularis, Bunch-rooted Crowfoot(3). Cabinet.

21 Ranunculus pennsyluanicus, Pennsylvanian Crowfoot(2). Cabinet.

222 R.antinculus recurvatus, Sanicle-leaved Crowfoot(2). Cabinet.

23 Ranunculus bulbosus, Buttercups(1). - - - Cabinet.

24 Caltha palustris,

25 Trollius laxus,

Common Marsh-marigold(3). Cabinet. American Globeflower(3). - Cabinet.

26 Coptis trifolia,

27 Helleborus viridis,

28 Aquilegia canadensis, Common Goldthread(4). - Cabinet. Green Hellebore(2). - - Cabinet. Canadian Columbine(2). - Cabinet. 29 Delphinium consolida, Common Larkspur(1). - - Cabinet.

30 Aconitum uncinatum,

31 Act正A RUBRA,

32 Actea alba, American Monkshood(1). - Cabinet. Red Cohosh(4). - - - - Cabinet.

33 Cimicifuga racemosa,

34 Thalictrum diorcum,

35 Thalictrum cornuti, White Cohosh(3). - - Cabinet.

36 Thalictrum anemonoides,

37 ZaNTHORRHIZA APIIFOLIA, Blacksnakeroot, or Rattleweed(4). Cabinet. Early Meadowme(3). - - Cabinet. Common Meadowrue(5). - Cabinet. Rue Anemone(8). - - - Cabinet. 38 Hydrastis canadensis,

Pearly-leaved Yellowroot(1). Cabinet. Canadian Yellowront(3). - Cabinet.

Vol. 2.] ORDER II. MAGNOLIACEA.

39 Magnolia glauca, Common Magnolia, or Sweet Bay(3). Cabinet. 40 Magnolia acdminata, Cucumber-tree(2). - - Cabinet. 41 Liriodendron tolipifera, Tulip Poplar(2). - - - Cabinet. ORDER III. ANONACEA.

42 Uvaria triloba, Papaw(2). - - - - Cabinet.

ORDER IV. MENISPERMACEA.

43 Menispermum canadense, Canadian Moonseed(3). - Cabinet.

ORDER V. BERBERIDACEA.

44 Berberis vulgaris, Barberry(3). - - - - Cabinet.

45 Leontice thalictroides, Blue Cohosh, or Pappoose-root(4). Cabinet. 
PLANTS.

Latin Names.

Einglish Names.

46 Teffersunia diphylita,

Rheumatism-root(3). - - Cabinet.

47 Podophylum peltatum, Mayapple, or Mandrake(1). - Cabinet.

ORDER VI. CABOMBACE $邓$.

48 Brasenia peltata, Watershield(2). - - - - Cabinet.

ORDER VII. NELUMBIACEA.

49 Nelumbium luteum, Great Yellow Walerlily.

ORDER VIII. NYMPHAACEA.

50 NyMPhIA ODORATA,

51 Nuphar lutea,

52 Nuphar adVENa,
Great White Waterlily(3). - Cabinet. Small-flowered Yellow Pondlily(4). Cabinet.

Common Yellow Pondlily(3). Cabinet.

ORDER IX. SARRACENIACEA.

53 Sarracenia purpurea, Common Sidesaddleflower(3). Cabinet.

ORDER X. PAPAVERACEA.

54. Sanguinaria canadensis, Bloodroot(5). - - - - Cabinet. 55 Chelidonium majus, Common Celandine(2). - - Cabinet.

ORDER XI. FUMARIACE $A$.

56 Dicentra cucullaria,

57 Dicentra canadensis,

58 Dicentra eximia,

59 AdLUMLA CIRRHOSA,

60 Corydalis aurea,

61 Corydalis gladea,

62 Fumaria officinalis,
Dutchman's-breeches(2). - - Cabinet. Squirrel-corn(4). - - - Cabinet. Choice Dicentra(2). - - - Cabinet. Climbing Fumitory(1). - - Cabinet. Golden Corydalis(3). - - - Cabinet. Glaucous Corydalis(3). - - Cabinet. Common Fumitory(2). - - Cabinet.
Vol. 3.]

63 Nasturtium palustre,

64 Nasturtium hispiduag,

65 Nasturtium Natans,

66 Barbarea vulgaris,

67 TURritis stricta,

68 ARABIS hiRsuTA,

69 Arabis dentiata,
ORDER XII. CRUCIFERAE.

Marsh Cress(4). - - - - Cabinet. Hispid Cress(3). - - - - Cabinet Floating Cress(2). - - - Cabinet. Scurvygrass(3). - - - - Cabinet. Straight Tower-mustard.

Hairy Wallcress.

Toothed Wallcress(1), - - Cabinet. 
Latin Names.

70 Arabis lyrata,

71 Arabis lefvigata,

72 Arabis canadensis,

73 Cardamine rhomboldea,

74 Cardamine pratensis,

75 Cardamine hirsuta,

76 Dentaria laciniata,

77 Dentaria DIPHylha,

78 Dentakia maxima,

79 SisymbriUM OFFICINALE,

80 Sisymbrium thalianum,

English Names.

Lyre-leaved Wallcress(2). - Cabinet.

Smooth Wallcress(2). - - Cabinet. Sicklepod(5). - - - - - Cabinet. Spring Cress(8). - - - - Cabinet.

Bittercress, or Cuckooflower(3). Cabinet. Watercress $(6)$. - - . - Cabinet. Common Toothwort(4). - - Cabinet. Pepper-root(4), - - - - Cabinet. Large Toothwort.

Common Hedge-mustard(2), - Cabinet. Mousear Cress(3). - - - Cabinet. 81 Erysimum cheiranthoides, Treacle Mustard(3). - - - Cabinet.

82 Sinafis Nigra,

83 Sinapis aRVENSIS,

84 Draba arabisans,

85 Draba caroliniana,

86 Draba verna,

87 Camelina sativa,

88 Thlaspi arvense,

89 Lepidium campestre, - Mithridate Peppervort(3). - Cabinet.

90 Lepidium ViRginicum,

91. Capsella bursa-Pastoris, Common Shepherdspurse(1). - Cabinet.

92 Cakile maritima, Sea Rocket(2). - - - - Cabinet.

93 Raphanus raphanistrum, Wild Radish(2). - - - - Cabinet.

\section{Vol. 4.] ORDER XIII. CAPPARIDACEAE.}

94 Polanisia gravfolens, Heavy-scented Polanisia(2). - Cabinet. ORDER XIV. VIOLACE

95 Viola pedata,

96 Viola palmata,

97 Viola cucullata,

98 Viola selKIRKII,

99 Viola sagittata,
Pedate Violet(4). - - - - Cabinet. Palmate Violet(3). - - - Cabinet. Hood-leaved Violet(2). - - Cabinet. Selkirk's Violet.

Arrow-leaved Vialet(7). - - Cabinet. 
Latin Names.

100 Viola rotundirolia,

101 Viola blanda,

102 VIOLA PRIMUL压FIIA,

103 Viola lanceolata,

104 Viola striata,

105 VIOLA MUHLENBERGiI,

106 Viola rostrata,

107 VIola PUBESCEns,

108 Viola CANADENSIS,

109 VIOLA TRICOLOR,

110 Solea CONCOLOR,
English Names.

Round-leaved Violet(5). - - Cabinet.

Sweetscented White Violet(5). Cabinet. Primrose-leaved Violet.

Lance-leaved Violet(6). - - Cabinet. Striated Violet(1). - - - Cabinet. Muhlenberg's Violet(6). - - Cabinet. Long-spurred Violet(4). - - Cabinet. Yellow Violet(3). - - - Cabinet. Canadian Violet(2). - - Cabinet. Pansey, or Heartsease(3). - Cabinet. Green-flowered Solea(1). - - Cabinet.

ORDER XV. CISTACE无.

111 Helianthemum canadense, Frostweed(9). - - - - Cahinet.

112 Lechea major,

113 Lechea thyMiFolia,

114 LECHEA MINOR,

115 Hudsonia ericoides,

116 Hudsonia tomentosa,
Larger Lechea(4). - - - Cabinet.

Thyme-leaved Lechea(2). - - Cabinet. Pinweed(7). - - - - Cabinet. Heath-like Hudsonia(1). - - Cabinet. Woolly Hudsonia(4). - - - Cabinet.

ORDER XVI. DROSERACEA.

117 Drosera rotundifolia,

118 Drosera longifolia,

119 Drosera filiformis,

120 Parnassia caroliniana,
Round-leaved Sundew(4). - Cabinet. Long-leaved Sundew(4). - - Cabinct. Thread-leaved Sundew(3). - Cabinet. Carolina Grass-of-Parnassus(3). Cabinet.

\section{ORDER XVII. HYPERICACEA.}

121 Ascyrum stans,

122 HypericUM PYRAMIDICUM,

123 Hypericum tralmianum,

124 HxpericUM PERForatUM,

125 Hypericum CORYMBosUm,

126 Hypericun ellipticum,

127 H.ypericum mutilum,
Upright St. Peter's-wort(1). Cabinet.

Giant St. John's-wort

Kalm's St. Joln's-wort(2). - Cabinet. Common St. John's-wort(2). - Cabinet. Corymbed St. John's-wort(3). Cabinet. Elliptical-leaved St. John's*urort(4). Cab. Small-flowered St. John's-wort(2). Cabinet. 
Latin Namer.

128 Hyfericum canadense,

129 HypericUm SAROTHRA,

130 Elodea virginica,
English Names.

Canadian St. John's-wort(4). Cabinet. Groundpine, or Nitueed(5). - Cabinet. Virginiun Elodea(3). - - - Cabinet.

\section{ORDER XVIII. ELATINACEA.}

131 ElatiNe aMERICANA, American Waterwort(4). - - Cabinet.

Vol. 5.] ORDER XIX. CARYOPHYLLACE As.

132 Honckenya peploides,

133 Sagina procumbens,

134 Sagina apetala,

135 Arenaria serpyldifolia,

136 Arenaria squarrosa,

137 ARENARIA stricta,

138 Arenaria grenlandica,

139 Memringia lateriflora,

140 Stellaaria media,

141 Stellaria loNgifolia,

142 Stellaria borealis,

143 Cerastium vulgatum,

144 Cerastium viscosom,

145 Cerastium arvense,

146 Cerastium oblongtfolium, Oblong-leaved Chickweed.

147 Cerastium nutans,

148 Silene stellata,

149 Silene antirrhina,

150 Silene NOCTIFLORA,

151 Silene penNsylvanica,

152 Silene virginica,

153 LychNis githago,

Common Sta Chickweed(3). - Cabinet. Procumbent Pearlworl(7). - Cabinet. Apetalous Pearlwort(10). - Cabinet. Thyme-leaved Sandwort(5). - Cabinet Squarrose Sanduoort(5). - - Cabinet. Upright Sanduort(5). - - Cabinet. Greenland Sandwort(3). - - Cabinet. Lateral-flowered Mahringia(5). Cabinet. Common Chickweed(2). - - Cabinet. Long-leaved Stitchwort(2). - Cabinet. Northern Stitchwort(5). - - Cabinet. Common Mousear Chickweed(1).Cabinet. Viscous Mousear Chickweed(3). Cabinet. Field Chickweed(3). - - - Cabinet. Nodding Chickweed(3). - - Cabinet. Four-leaved Campion(4). - - Cabinet. Snapdragon Catchfy(2). - - Cabinet. Night-flowering Catchfly(1). Cabinet. Wild Pink(3). - . - - - Cabinet. Virginian Catchfly(1). - - Cabinet. Rose Campion, or Corncockle(2).Cabinet. 154 Saponaria officinalis, Common Soapuort, or Bouncing-Bet(2).Cab. 155 Saponaria vaccarla, Perfoliate Soapwort. 156 Mollugo verticildata, Carpetweed(3). - . - . Cabinet. 
ORDER XX. ILLECEBRACE $A$.

Iatin Names.

157 ANYCHIA DICHOTONA,

158 Spergula arvensis,

159 Spergula rubra,

160 Scleranthus annuUs,
English Names.

Common Forked Chickweed(4). Cabinet.

Corn Spurry, or Tare(3). - Cabinet.

Red-flowered Spurry(6). - - Cabinet.

Common Knawel(2). - - Cabinet.
Vol. 6.]

ORDER XXI. PORTULACACEA.

161 Portulaca oleracea,

162 Craytonia virginica,

163 Claytonia caroliniana,
Common Purselane(1). - - Cabinet.

Narrow-leaved Spring-beauty(6). Cab.

Broad-leaved Spring-beauty(6). Cabinet.

ORDER XXII. MALVACEA.

161 Malva rotundifolia,

165 Malva sylvestris,

166 Althexa officinalts,

167 Abutilon aVicenn.e,

168 Sida SPINOSA,

169 Hibiscus virginicus,

170 Hibiscus moscheutos,

171 Hibiscus trionum,
Dwarf or Running Mallow(1). Cabinet. High Mallow.

Common Marshmallow.

Velvetleaf(1). - - - - Cabinet.

Prickly Sida(1). - - - Cabinet.

Sweating-weed(1). - - - Cabinet.

Mallowrose(1). - - - - Cabinet.

Venetian Mallow(1). - - Cabinet.

ORDER XXIII. TILIACEA.

172 Tilia americana,

Basswood(2). - - - - Cabinet.

ORDER XXIV. LINACEA.

173 Linum virginianum,

Wild Flax(5). - - - - Cabinet.

174 Linum usitatissimum,

Common Flax.

ORDER XXV. GERANIACEAE.

175 Geranium maculatom, Spotted Cranesbill(2). - - Cabinet.

176 Geranium caroliniandm, Carolinian Cranesbill(4). - Cabinet.

177 Geranium pusillum, Small-flowered Cranesbill(5). Cabinet.

178 Geranium robertianum, Herb-robert(1). - - - - Cabinet.

179 Erodium cicutarium, Hemlock Heronsbill(2). - - Cabinet. 
ORDER XXVI. OXALIDACEA.

Latim Names.

180 Oxalis ACETOSELLA,

181 Oxalis violacea,

182 Oxalis stricta,
English Names.

Common Woodsorrel(5). - - Cabinet.

Violet Woodsorrel(6). - - - Cabinet.

Yellow Woodsorrel(4). - - Cabinet.

ORDER XXVII. BALSAMINACEA.

183 Impatiens PaLtida,

184 Impatiens FUlva,
Touch-me-not, or Snapweed(1). Cabinet.

Balsam-zeed, or Jewel-weed(2). Cabinet.

ORDER XXVIII. LIMNANTHACEAE.

185 Floskea proserpinacoides, False Mermaid(3). - - - Cabinet.

ORDER XXIX. ANACARDIACE $Z$.

186 Rhus TyPhina,

187 Rhus glabra,

188 Rhus copajLina,

189 Rhus venenata,

190 Rhus toxicodendron,

191 Rhus aromatica,
Stagshorn Sumach(2). - - Cabinet.

Smooth Sumach(1). - - Cabinet.

Mountain Sumach(4). - - Cabinet.

Poison Sumach(3). - - - - Cabinet.

Poisonvine, or Mercury(4). - Cabinet.

Sweet-scented Sumach(2). - Cabinet.

ORDER XXX. XANTHOXYLACEA.

192 Zanthoxylum americanum, Common Prickly-ash(4). - Cabinet. 193 Ptelea thifoliata,

Swamp Dogwood(2). - - Cabinet.

Vol. 7.]

194 ACER PENNSYlvanicum,

195 AcER spicatum,

196 Acer saccharinum,

197 ACER Dasycarpum,

198 ACER RUBRUM,
ORDER XXXI. ACERACEA.

Striped Maple(6). - - - - Cabinet.

Mountain Maple(1). - - - Cabinet.

Sugar Maple(3). - - - - Cabinet.

White Maple(2). - - - - Cabinet.

Red Maple(9). - - - - - Cabinet.

ORDER XXXII. HIPPOCASTANACE $A$.

199 Asculus hippocastantu, Common Horse-chesnut(1). - Cabinet.

ORDER XXXIII. CELASTRACEA.

200 Staphylea trifolia,

201 Celastrus scandens,

202 Euonymus atropurpureus, Burning-bush(2). - - - - Cabinet.

203 EUONYMUS AMERICANUS,
American Bladdernut(4). - Cabinet. Bittersweet(3). - - - - - Cabinet.

Strawberry-tree(2). - - C Cabinet. 


\section{ORDER XXXIV. RHAMNACEA.}

Latin Names.

204 Rhaimnus cathaRticus.

205 Rhamnus almifolius,

206 Ceanothus americanus,

207 Cfanothus ovalis,
English Names.

Common Buckthorn.

Alder-leaved Buckthorn(3). - Cabinet. New-Jersey Tea, or Redroot(3). Cabinet. Narrow-leaved Ceanothus.

ORDER XXXV. VITACE无.

208 Vitis LABRUSCA,

209 Vitis enstivalis,

210 Vitis cordifolia,

211 Vitis RIPARIa,
Fox Grape(4). - - - - Cabinet.

Summer Grape(1). - - : - Cabinet. Frost Grape(3). - - - - Cabinet,

Winter Grape(1). - - - - Cabinet,

212 Ampelotsis quinquefolia, Virginian Creeper(3). - - Cabinet.

ORDER XXXVI. POLYGALACEA.

213 Polygala sanguinea,

214 Polygala cruclata,

215 Polygala verticillata, 216 Polygala ambigua,

217 Polygala senega,

218 Polygala polygama,

219 Polygaja yauctrolia,
Purple Milkwort(3). - - - Cubinet, Cross-leaved Milkwort(3), - Cabinet. Whorl-leaved Milkwort(3). - Cabinet. Ambiguous'Milkwort(4). - - Cabinet. Seneca Snakeroot(1). - - Cabinet. Polygamous Milkwort(3). Cabinet. Fringed Milkwort(3). - - Cabinet.
Vol. 8.]

220 VICIA AMERICANA,

221 ViCIA CRACCA,

222 Vícia tetrasperma,

223 VICIA SATIVA,

224 Ervum hirsutom,

225 Lathyrus MARITIMUS,

226 Lathyrds ochroledcus,

227 LATHYRUS MYRTIFOLIUS,

228 Lathyrds palustris,

229 Phaseolus perennis, 230 Phaseolus diversifolius, Varione-leaved Kidneybean(2). Cabinet,
American Vetch(1). - - - Cabinet. Tufted Vetch(1). - - - - Cabinet. Slender Vetch(2). - - - - Cabinet. Common Vetch(3). - - - - Cabinet. Hairy Tare(2). - - - - - Cabinet. Seaside Vetchling(3). - - - Cabinet. Cream-colored Vetchling(1). - Cabinet. Myrtle-leaved Vetchling(3). - Cabinet. Marsh Velchling(2). - - - Cabinet. Perennial Kidneybean(2). - Cabinet. 
Latin Names.

231 Phaseolus helvolus,

232 Aptos tuberosa,

233 Galactia glabella,

234 Clitoria mariana,

235 AMpHicarpora monotCa,

236 Robinia pseudacacia,

23T Tephrosia virginiana,

238 Trifolium arvense,

239 Trifolium pratense,

240 Trifolium ReFlexum,

241 Trifolium repens,

242 Trifolitim agrarium,

243 Melilotus officinalis,

244 Melilotus heitcantha,

245 Medicago sativa,

246 Medicago lupuhina,

247 Astragalus canadensts,

248 Phaca negiecta,

249 Stylosanthes elatior,

Vol. 9.]

250 Desmodium nudiflorum,

251 Desmodium acuminatum,

252 Desmodium canadense,

253 Desmodium canescens,

254 Desmodiun dilienis,

255 Desmodium cuspidatum,

256 Desmodium viridiflorum, Velvet-leaved Desmodium(3). Cabinet.

257 Desmodium marilandicum, Smooth Small-leaved Desm.(2). Cahinet. 258 Desmodiún cXítare,

259 Desmodium Rigidum, Hairy Small-leaved Desm.(1). Cabinet. Rigid Desmodium(1). - - - Cabinet.

260 Dessmodium paniculatum, Panicled Desmodium(2), - - Cabinet. 261 Desmodium rotundifolium, Round-leaved Desmodium(1). Cabinet.

Long-slalked Kidneybean(2). Cabinet. Groundnut(5). - - - - Cabinet. Smooth Milkvine.

Maryland Clitoria(3). - - Cabinet. Common Hognut(4). - - Cabinet.

Common Locust-tree(1). - - Cabinet. Goat's-rue, or Catgut(2). - - Cabinet. Stone Clover(6). - - - Cabinet. Red Clover(3). - - - - Cabinet. Buffalo Clover(1). - - - Cabinet. White Clover(2). - - - Cabinet. Yellow Clover(1). - - - Cabinet. Yellaw Melilot(1). - - - Cabinet. White Melilot(3). - - - Cabinet. Spanish Trefoil, or Lucern.

Black Medick, or Nonesuch(3). Cabinet. Canadian Milkvetch(3). - C Cabinet. Bastard Vetch(3).. - - - Cabinet. Pencilfower(4). - - - Cabinet.

Naked-flovsered Desmodium(3). Cabinet. Pointed-leaved Desmodium(2). Cabinet. Canadian Desmodium(4). - Cabinet. Hoary Desmodium(2). - - Cabinet. Dillenius's Desmodium(3). - Cabinet. Large-bracted Desmodium(3). Cabinet. 
Latin Names.

262 Lespedeza Procumbens,

263 Lespedeza Repens,

264 Lespedeza violacea,

265 Lespedeza hirta,

266 Lespedeza capitata,

267 Genista tinctoria,

268 Crotalaria sagiptalis,

269 LUPINUS PERENNIS,

270 Baptisia tinctoria,

271 Baptisia australis,

272 Cercis canadensis,

273 Cassia maritandica,

274 CașSia Chamercrista,

.275 Cassia nictitans,

276 Gymnocladus canadensis, Coffee-tree(1). - - - Cabinet.

277 Gleditschia triacanthos, Honey Locust(4). - - - Cabinet.

Vol. 10.]

ORDER XXXVIII. ROSACEEA.

278 Prunus americana,

279 Prunus maritima,

280 Cerasus pumita,

281 Cerasts pennsquvanica,

282 Cerasus virginiana,

283 Cerasus serotina,

284 Spirat opulifolia,

285 Spir fa salicifolia,

286 Spirea tomentosa,

287 SPIRAA ARUNCUS,

288 Gillenia trifoliata,

289 Gillenia stipdlacea,

290 Geum virginianum,

291 Geum strtctum,

292 Ggum RIVALE,
English Names.

Trailing Lespedeza(2), - - Cabinet.

Slender Lespedeza(2). - - - Cabinet.

Bush Clover(7). - - - - Cabinct.

Hairy Lespedeza(3). - - - Cabinet.

Round-headed Lespedeza(5). - Cabinet.

Dyer's Greenweed.

Small Annual Rattlebox(4). - Cabinet.

Common Wild Lupine(1). - Cabinet.

Common Wild Indigo(3). - Cabinet.

Blue-flowered False Indigo,

American Judas-tree(1). - - Cabinet.

American Serna(2). - - Cabinet.

Sensitive Pea(1). - . - - Cabinet.

Sensitive-plant(2). - - Cabinet.

Red or Yellow Plum(2). - = Cabinet.

Beach or Sand Plum(6). - - Cabinet.

Sand Cherry(2). - - - Cabinet.

Bird Cherry(2). - - - Cabinet.

Chokecherry(3). - - - Cabinet.

Wild Cherry(2)- - - - Cabinet.

Ninebark(2). - - - - Cabinet,

Queen-of-the-meadow(2). - - Cabinet.

Hardhack, or Steeplebush(2)." Cabinet.

Goatsbeard.

Indian-physic, or Bowman's-root(2). Cabinet. American Ipecacuanha(1). - Cabinet, Virginian Avens(2). - - Cabinet, Small-flowered Yellow Avens(1). Cabinet. Water Avens(2). - - - Cabinet, 
Latin Names.

293 Geum triflorum,
English Names.

Three-flowered Purple Avens(2). Cabinet.

294 Waldsteinia fragarioides, Strawberry-like Waldsteinia(2). Cab.

295 Sanguisolba canadensis, American Great Burnet(3). - Cabinet. 296 Agrimonta eupatoria, Common Agrimony(2). - - Cabinet. 297 Potentilia norvegica, Norwegian Cinquefoil(2). - Cabinet. 298 Potentilla tridentata, Three-toothed Cinquefoil(4). - Cabinet. 299 Potentilla canadensis, Common Cinquefoil, or Fivefinger(2). Cab. 300 Potentilla argentea, Silver-leaved Cinquefoil(1). - Cabinet. 301 Potentilla arguta, Close-flowered Cinquefoil)1). - Cabinet. 302 Potentilla fruticosa, 303 Potentilla anserina, 304 Comarum palustre, 305 Fragaria virginiana, 306 Fragaria vesca, 307 Dalibarda repens, 308 Rubus odoratus, 309 RUBUS TRIFLORUS, 310 RUBUS Strigosts, Shrubby Cinquefoil(3). - - Cabinet. Wild Tansey, or Silverweed(5). Cabinet. Common or Marsh Cinquefoil(1). Cabinet. Wild Strawberry(1). - - - Cabinet. Wild Strawberry(3). - - - Cabinet.. Creeping Dalibarda(4). - - Cabinet. Flowering Raspberry(1). - - Cabinet. Dwarf Rasplerry(4). - - Cabinet. Red Wild Raspberry(2). - - Cabinet. 311 Rubus occịdentalis, Black Raspberry, or Thimbleberry(3). Cabinet.

312 Rubus villosus,

313 RUBUS Canadensis,

314 RUBUS HISPIDUS,

315 Rugus cuneifolius, 316 Rosa carolina, 317 Rosa LUCIDA, 318 Rose BLANDA, 319 Rosa rubiginosa, 320 Crategus oxycantha, 321 Crategus crus-gali, 322 Cratefgus coccinea, 323 Cratefuto tomentosa, 324 Crategus punctata,
Common Blackberry(4). - - Cabinet. Low Blackberry, or Dewberry(1). Cabinet. Running Swamp Blackberry(7). Cabinet. Sand Blackberry(3). - - Cabinet. Swamp Rose(1), - . - Cabinet. Dwarf Wild Rose(6). - - - Cabinet. Early Wild Rose(2). - - - Cabinet. Sweetbriar, or Eglantine(2). Cabinet. Hawthorn, or English Thorn(4). Cabinet. Cockspur Thorn(3). - - - Cahinet. White Thorn(4). - - - Cabinet. Black Thorn(2). - - - Cabinet. Common Thorn(2). - - Cabinet. 
Latin Names.

325 Pyrus coronaria,

326 Pyrus arbutifolia,

327 Pyrus americana,

328 Amelanchier canadensis, Common Juneberry(6). - Cabinet.
Vol. 11.]

ORDER XXXIX. MELASTOMACEA.
Meadow-beauty, or Deergrass(3). Cabinet,

\section{ORDER XL. LYTHRACEA.}

330 Ammannia mumilis, Dwarf Ammannia.

331 LYTHRUM HYSSOPIFOLIA, 332 Decodon verticiliatum, 333 Cuphea viscosissima,
Common Purple Loosestrife.

Swamp Willowherb(2). - - Cabinet. Viscid Cuphea.

\section{ORDER XLI. ONAGRACEA.}

334 Eilobium angustifolium, Rosebay Willouherb(2). - - Cabinet.

335 Epilobium alpinum,

336 Epilobium coloratum,

337 Epilobium molle,

338 Epilobium palustre,

339 Ginothera bienNis,

340 Ginothera Fruticosa,

341 ENothera LINEARIS,
Alpine Willowherb(2). - - Cabinet.

Purple-leaved Willowherb(2). Cabinet. Soft Willowherb.

Narrow-leaved Willowherb(2). Cabinet.

Common Evening Primrose(2). Cabinet. Sundrops(2). - - - - Cabinet.

Narrow-leaved Evening Primrose.

342 CEnothera chrysantha, Golden-flowered Evening Primrose.

343 Enothera pUmila,

344 GaUra biennis,

345 Ludwigia alternifolia,

346 LUDWIGIA SPHEROCARPA,

347 Ludwigia palustris,

348 Circ.za lutetiana,

349 Circ $x$ a alpina, Common Enchanter's Nightshade(2). Cabinet.

350 Proserpinaca palustris, Marsh Mermaid-ukeed(4). Cabinet.

351 Proserpinaca pectinacea, Cut-leaved Mermaid-weed(3). Cabinet. 352 Myriophylium spicatum, spiked Water-milfoil.

Dwarf Evening Primrose(1). Cabinet. Biennial Gaura(3). - - Cabinet. Seedbox(3). - - - - C Cabinet. Round-fruited Ludwigia(1). Cabinet. Water Purselane(2). - - - Cabinet. Alpine Enchanter's Nightshade. 
Latin Names.

English Names.

353 Myriophylum ver ticillatum, Whorled Water-milfoil(1). Cabinet. 354 Myriophylum heterophylum, Various-leaved,Water-milf:(2).Cab. 355 Mrriophylud ambigudr, Polymorphous Water-milfoil(1). Cabinet. 856 Myrophylium tenellum, Leafless Water-milfoil(5). - Cabinet. 357 Hiprunis vulgaris, Common Marestail(2). - - Cabinet.

ORDER XLII. CACTACE

358 Opuntia vulgaris, Common Indian-fig, or Prickly-pear(7). Cabinet.

ORDER XLIII. GROSSULACEAE.

359 Ribes cynosbati, 360 Ribes RotUNDIFoliUm, 361 Ribes lacustre, 362 Ribes Floridum, 363 Ribes prostratum,
Prickly Gooseberry(6). - - Cabinet. Round-leaved Gooseberry(2). Cabinet. Swamp Gooseberry(2). - - Cabinet. Wild Black Currant(2). - - Cabinet. Fetid Currant(2). - . - Cabinet.

ORDER XLIV. CUCURBITACEA.

364 Sxcyos angulatus, Common Single-seeded Cucumber(1). Cabinet. 365 Echinocystis lobata, Wild Balsamapple(2). - Cabinet.

ORDER XLV. CRASSULACEA.

366 Thlexa simplex,

367 SEDUM TELEPHIOIDES, 368 Sedt M TELephium, 369 Penthordm sedoides,
Pigmyweed.

American Orpine.

Liveforever(2). - - - - Cabinet.

Virginian Stonecrop(1). - - Cabinet.

ORDER XLVI. SAXIFRAGACE列.

370 Saxifraga virginiensis, Virginian Saxifrage(1). - - Cabinet. 371 Saxifraga pennsylvanica, Pennsylvanian Saxifrage(4). Cabinet. 372 HEUCHERA AMERICANA, Common Alumroot. 373 Mitella Diphylla, 374 Mitella NUDA, 375 Tiarella cordifolia, Common Bishopscap(3). - - Cabinet. Stoloniferous Bishopscap(4). Cabiret. Heart-leaved Mitrewort(1). - Cabinet. 376 Chrysosplenium americanum, Golden Saxifrage(8). - - Cabinet.

ORDER XLVII. HAMAMELACEA. Witch Hazel(2). - - - - Cabinet. 
Vol. 12.] . ORDER XLVIII. UMBELLIFERA. Latin Names. English Names. '

378 Hydrocotyle americana, American Marsh-pennywort(4). Cabinet. 379 Hydrocotyle umbeldata, Many-flowered Mar-sh-pennywort(4).Cab.

380 Crantzia lineata,

381 Sanicula marilandica,

382 Sanicula canadensis,

383 Discopleura capillacea, Few-rayed Discopleura(2). - Cabinet. 384 Bupleurum rotundifolium, Thoroughwax, or Modesty(1). Cabinet. 385 Cicuta maculata, Water-hemlock, or Spotted Cowbane(3). Cabinet.

386 Cicuta bulbifera,

387 Sium Latifolium,

388 SiUm LINEARE,

389 Cryptot enia canadensis, Common Honewort(2). - - Cabinet.

390 Zizia CORDATA,

391 Zizia aUREA,

392 Zizia INTEgerRIMA,

393 Thaspium atropurpureum, Purple Alexanders(1). - - Cabinet.

394 Thaspium aUReUM,

395 Thaspium barbinode,

396 Ethusa cxnapium,

397 Conioselinum canadense, Canadian Conioselinum(4). - Cabinet. 398 Archangelica atropurpurea, Common Angelica.(7). - Cabinet. 399 Archangelica hirstta, 400 Archemora RIgIDA, 401 Pastinaca sativa, 402 Heracleum lanatum, 403 Daucus carota, 404 OSMORHIZA LONGISTYLIE, 405 OsMorhiza BREvistylis, 406 Coniụm maculatum, 407 Erigenia bulbosa,
Bulbiferous Water-hemlock(4). Cabinet. Broad-leaved Water-parsnep(4).Cabinet. Narrow-leaved Water-parsnep(4).Cabinet. Heart-leaved Alexanders(4). - Cabinet. Golden Meadow-parsnep(1). - Cabinet. Entire-leaved Zizia(2). - - Cabinet. Golden Thaspium(3). - - Cabinet. Hairy-jointed Thaspium(3). - Cabinet. Common Fool's Parsley(2). - Cabinet. Downy Angelica(3). - - - Cabinet. Rigid-leaved Archemora(5). - Cabinet. Cornmon or Wild Parsnep(3). Cabinet. American Cow Parsnep(5). - Cabinet. Common or Wild Carrot(2). Cabinet. True Sweet Cicely(2). - - - Cabinet. Spurious Sweet Cicely(4). - Cabinet. Common Poison Hemlock(4). Cabinet. Bulbous-rooted Erigenia(4). Cabinet. 
Vol. 13.]

Latin Names.

408 Aralia racemosa,

409 Aralia nudicaulis,

410 Aralia hispida,

411 Panax quinquefolium,

412 Panax trifolium,
ORDER XLIX. ARALIACEA.

English Names.

Spikenard(1). - - - - - Cabinet.

Wild Sarsaparilla(1). - - Cabinet.

Wild Elder(1). - - - - Cabinet.

Common Ginseng(4). - - - Cabinet.

Dwarf Ginseng(4). - . - Cabinet.

ORDER L. CORNACEA.

413 Cornus alternifolia,

414 Cornus circinata,

415 Cornus stolontfera,

416 Cornus paniculata,

417 Corndos Sericea,

418 CoRNUS FLORIDA,

419 Cornus canadensis,
Alternate-leaved Dogwood(2). Cabinet. Round-leaved Dogwood(2). - Cabinet. White-berried Dogusood(2). - Cabinet. Paricled Dogwood(3). - Cabinet. Swamp Dogwood(3). - - Cabinet. Common Dogwood(4). - . - Cabinet. Dwarf Dogwood(3). - - - Cabinet.

\section{ORDER LI. CAPRIFOLIACEA.}

420 LINN

Two-flowered Linnaa(4). - - Cabinet.

421 Symphoricarpus Racemosa, Common Snowberry(2). - - Cabinet. 422 Symphoricarpus vulgatis, Indian Currant(2). - . - Cabinet. 423 Lonicera sempervirens, Trumpet Honeysuckle(4). - Cabinet.

424 Lonicera grata,

425 Lonicera flava,

426 LoNicera hirsuta,

427 LoNicera parViflora,

428 Lonicera ciliata,

429 LONICERA CARULEA,

430 LONICERA OBLONGIFOLIA,

431 Diervilla trifida,

432 Triosteum perfoliatum,

433 Sambucus pubens,

434 Sambuctus canadensis,

4.35 Viburntim NUDUM,
Wild Honeysuckle.

Yellow Honeysuckle.

Hairy Honeysuckle(4). - - Cabinet. Small-flowered Honeysuckle(5). Cabinet. Fly Honeysuckle(4). - - - Cabinet. Hairy Fly Honeysuckle(1). - Cabinet. Long-stalked Honeysuckle(1). Cabinet. Common Bush Honeysuckle(1). Cabinet. Feverwort, or Horse Gentian(1). Cabinet. Red-berried Elder(2). - - - Cabinet. Common Elder(2). - - C Cabinet. Swamp Viburnum(7). - - - Cabinet. 
Latin Names.

436 VibURnum PRUNIFOLITM,

437 Vibunnum lentago,

438 ViruRnUm DENTATUM,

439 Viburnum pUbescens,

- 440 Viburnum acerifolium, 441 Viburnum padCiflorum, 442 Viburnum opUlus, 443 Viburnum LaNTaNoIdes,
English Names.

Black Haw, or Sloe(1). - - Cabinet. Sweet Viburnum(2). - - - Cabinet. Arrow-wood(3). - - - - Cabinet. Pubescent Viburnum(4). - - Cabinet. Maple-leaved Arrow*wood(3). Cabinet. Mountain Bush Cranberry(1). Cabinet. High Cranberry(2). - - Cabinet. Hobblebush(3). - - - - Cabinet.
Vol. 14.]

444 Galium aparine,

445 Galium trifidum,

446 Galium asprellum,

447 Galium triflorum,

448 Galium pilosum,

449 Galium circazans,
450 Galium boreale,

ORDER LII. RUBTACE E.

Common Cleavers, or Goosegrass(1). Cabinet. Small Bedstravo(9). - - - Cabinet. Rough Bedstraw(2). - - - Cabinet.

Sweetscented Bedstraw(1). - Cabinet. Hairy Bedstravo(3). - - - Cabinet. Wild Liquorice(5). - - - Cabinet. Northern Bedstraw(2). - - Cabinet. 451. Ceprialanthus occidentalis, Butterbush, Pond Dogwood(2). Cab. 452 Mitchelia Repens, 453 Hedyotis CERULEA, 454 Hedyotis ciliolata, 455 Hed yotis LoNGIFolia, 456 Hedyotis glomerata, Partridgeberry(3). - - - Cabinet. Common Bluets, or Dwarf Risk(5). Cabinet. Fringed-leaved Bluets(4). - Cabinet. Long-leaved Bluets(6). - - Cabinet. Cluster-flowered Bluets.

ORDER LIII. VALERIANACEA.

457 Valeriana sylvatica,

458 FEDia Fagopyrom,
Tall Swamp Valerian(2). - Cabinet.

Buckwheat Cornsalad(3). - Cabinet.

\section{ORDER LIV. DIPSACEAT.}

459 Dipsacus sylvestris,

Wild Teasel(2). - - - - Cabinet.

Vol. 15.]

ORDER LV. OOMPOSITA.

460 Vernonia noveboracensis, Common Ironweed(3). - - - Cabinet. 461 Liatris cýliNdRACEA, Small Buttonsnakeroot(1). - Cabinet. 462 Liatris spicata, Tall Buttonsicakeroo'(1). - . Cabinet. 
Latin Names.

-463 Liatris scariosa,

464 Eupatorium purpureum, Joe-Pye-weed(6). - - - - Cabinet, 465 Eupatorium leucolefis, White-scaled Hempweed.

466 Eupatoridm teucrifolium, Germander-leaved Hempweed(2).Cabinet, 467 Eupatorium rotundifolium, Round-leaved Hempweed.

468 Eupatorium sessilifolium, Upland Boneset(1), - - - Cabinet.

469 Eupatorium Perfoliatom, Boneset, or Thoroughwort(1). Cabinet. 470 Eupatoridm ageratoides, White Srakeroot(1). - - - Cabinet. 471 Eupatoridm aromaticum, Sweetscented Hempweed(2). - Cabinet. 472 Mikania scandens, Common Climbing Hempweed(3). Cabinet. 473 Nardosmia Falmata, 474 Tessilago farfara, Common Coltsfoot(5). - - Cabinet. 475 Sericocarpus conyzoides, Broad-leaved Sericocarpus(2). Cabinet. 476 Sericocarpus solidagineds, Narrow-leaved Sericocarpus(2).Cabinet.

Vol. 16.]

477 Aster corymbosus,

478 Aster MacrophyluUs,

- 479 Aster radula, 480 Aster spectabilis, 481 Aster patens, 482 Aster I 483 Aster undulatus, 484 Aster cordifolius, 485 Aster sAgITTIFoliUs, 486 Aster ericoides, 487 Aster multiflor ds, 488 Aster dumosus, 489 Aster tradescanti, 490 Aster miser, 491 Aster simplex, 492 Aster tendifolios, 493 Aster GREENI,
Corymbed Aster(2). - - - Cahinet. Large-leaved Aster(1). - - Cabinet. Rasp-leaved Aster.

Showy Aster.

Spreading Aster(2). - - - Cabinet.

Smooth Blue Aster(1). - Cabinet.

Various-leaved Aster(3). - - Cabinet. Heart-leaved Aster(1). - - Cabinet. Arrow-leaved Aster.

Heath-like Aster.

Many-flowered Aster(2). - - Cabinet. Bushy Aster(1). - - - - Cabinet. Tradescant's Aster(2). - - Cabinet. Starved Aster.

Willow-leaved Aster(4). - - Cabinet. Slender-leaved Aster.

Green's Aster. 
Latin Names.

494 Aster laxus,

495 Aster elodes,

496 AsteR FUNICEUS,

497 Aster PRENANTHOIdES,

498 Aster Nove-ANGLI灰,

499 Aster acuminatus,

500 Aster ptarmicoides,

501 Aster flexuosus,

502 Aster LINIFolius,
English Names.

Loosely-branched Aster.

Blue Smooth Marsh Aster(1). Cabinet. Hispid Tall Aster(5). - - Cabinet.

Prenanthes-like Aster(1). - Cabinet. New-England Aster(2). - - Cabinet. Acuminate Aster(2). - - - Cabinet. Ptarmicoid Aster(2), - - - Cabinet. Perennial Salt-marsh Aster(1). Cabinet. Annual Salt-marsh Aster(3). Cabinet. 503 Diplopappus limariffolius, Narrow-leaved Diplopappus(6). Cabinet. 504 Diplopappus cornifolius, Cornel-leaved Diplopappus(4). Cabinet. 505 Diplopappus umbellatus, Umbelled Diplopappus.

506 Erigeron canadense, Horseweed, or Butterweed(1). Cabinet. 507 Erigeron bellidifolium, Poor Robert's Plantain(2). - Cabinet. 508 Erigeron philadelphicum, Philadelphia Fleabane(2). - Cabinet. 509 Erigeron anNuUm, Sweet Scabious, Daisy, \&c.(1). Cabinet. 510 Erigeron strigostm, Fleabane(1). - - - - Cabinet.

Vol. 17.]

511 Solidago squarRosa, 512 SOLIDAGO BICOLOR, 513 Solidago latifolia, 514 Solidago cesia, 515 Solidago stricta, 516 SoldDago VIRGA-AUREA, 517 Solidago RIGIDA, 518 SOLIDAGO OHIOENGIS,

519 Solidago semperviring, 520 SoLidago NEgLECTA,

521 Solidago Patula, 522 Bolidago arfita, 523 Solidago muhlenbergi, 524 Soridago altissima,
Squarrose Goldenrod(1). - - Cabinet. White Galdenrod(4). " - - Cabinet. Broad-leaved Goldenrod(4). " Cabinet. Blue-stemmed Goldenrod(3). Cabinet. Willow-leaved Goldenrod(4). Cabinet. European Goldenrad(3). - - Cabinet. Rigid-leaved Goldenrod(2). " Cabinet. Ohio Goldenrad(2). - - - Cabinet. Salt-marsh Goldenrod(2). - Cabinet. Neglected Goldenrod(1). - - Cabinet. Spreading Goldenrod(3). - - Cabinet. Sharp-tootked Goldenrod(1). Cabinet. Muhlenberg's Goldenrod(2). - Cabinet. Tall Hairy Goldenrod(1). - Cabinet, 
Latin Names.

525 Solidago Ulmifolia,

526 Solidago odora,

527 Soliddago nemoralis,

528 Solidago canadensis,

529 Solidago serotina,

530 Solidago gigantea,

531 Solidago laNceolata,

532 Solidago tenUifolia,

533 Chrysopsis falcata,

534 Chrysopsis mariana,

535 Baccharis halimifolia,

536 Pluchea camphorata,

537 InUla helenium,

Vol. 18.]

538 Polymnia canadensis,

539 Polymina uvedalia,

540 Silphim trifoliatum,

541 Iva FRUTESCENS,

542 Ambrosia trifida,

543 Antrosta artemisi fro
544 Xanthium strumaridm,
545 Xanthium Echinatum,

546 XanTHIUM SPINOSUM,

547 Heliopsis Lervis,

548 RUdBECKIA HIRTA,

549 RUdBeckia laciniata,

550 Lepachys pinnata,

551 Helianthus giganteus,

552 Helianthus strumosus,

553 Helianthus decapetalus, Wild Sunflower(1). - - Cabinet.

554 Hedianthus divaricatus, Small Rough-leaved Sunflower(3).Cabinet.

555 Helianthus tuberosus, Jerusalem Artichoke(1). - - Cabinet.

English Names.
Elm-leaved Goldentrod.

Sweetscented Goldenrod(2). - Cabinet. Gray Goldenrod(3). - - - Cabinet.

Canadian Goldenrod(1). - - Cabinet.

Late-flowering Goldenrod(2). Cabinet.

Tall Smooth Goldenrod(1). - Cabinet.

Bushy Goldenrod(1). - - - Cabinet.

Slender-leaved Goldenrod(2). Cabinet.

Sickle-leaved Chrysopsis(2). - Cabinet.

Maryland Chrysopsis(3). - - Oabinet.

Groundselbush(1). - - - Cabinet.

Seaside Marsh-fleabane(2). - Cahinet.

Common Elecampane(1). - - Cabinet.

Small-flowered Leafcup(3). - Cabinet. Large-flowered Leafcup(2). - Cabinet. Ternate Silphium.

Highwater-shrub(2). - - Cabinet.

Three-lobed Ragweed(2). - - Cabinet. Common Cocklebur(1). - Cabinet. Sea Cocklebur(2). - - - Cabinet. Thorny Cocklebur(1). - - Cabinet. Oxeye(1). - - - - - Cabinet. Large Hairy Rudbeckia(2). - Cabinet. Tall Smooth Rudbeckia(2). - Cabinet. Tall Lepachys(2). - - - - Cabinet. Tall Wild Sunflower(4). - . Cabinet. Wild Sunflower(2). - - Cabinet. 
Latin Names.

Euglish Names.

556 Actinomeris squarrosa, Squarrose Actinomeris(1). - Cabinet.

557 Coreopsis thichosperma, Tickseed Sunflonver(1). - - Cabinet.

558 Coreopsis RoseA,

Small Rose-colored Coreopsis(1).Cabinet.

559 Bidens frondosa, Common Bur-marigold, or Sticktight(2).Cabinet.

560 Bidens connata,

Swamp Beggarticles(2). - - Cabinet.

561 Bidens CERnuA,

Swamp Beggarticks.

562 Bidens chrysanthemoides, Large-flowered Bur-marigold(1). Cab.

563 Bidens Beckir,

564 Bidens bipinnata,

Water Marigold(2). - - - Cabinet.

565 Helentum autumale,

Spanish-needles(2). - - - Cabinet.

Sneezeweed(2). - - - - - Cabinet.

Vol. 19.]

566 Maruta cotula,

Fetid Chamomile, or Mayweed(1). Cabinet.

567 ANTHEMIS aRVENSIS,

Wild Chamomile(1). - - - Cahinet.

568 ACHILLEA MILLEFoLIUM,

Common Yarrow(1). - - Cabinet.

569 Leucanthemum vulgare, Whileweed, or Daisy(2). - - Cabinet.

570 Tanacetura volgake,

Common Tansey(1). - - Cabinet.

571 Artemisia canadensis,

572 Artemisia caudata,

573 Artemisia vulgaris,

Wild Wormwood(1). - - Cabinet.

Tall Biennial Wormwood(2). Cabinet.

574 Gnaphalium decurrens, Decurrent Cudweed(1). - - Cabinet.

575 Gnaphalium polycephalum, Life-everlasting(2). - - - Cabinet.

576 Gnaphalidm uliginosum, Marsh Cudweed(2). - - Cabinet.

577 Gnaphaitum purpureum, Purple Cudweed(4). - - - Cabinet.

578. Antennaria margaritacea, Pearly Everlasting(2). - Cabinet.

579 antennaria plantaginifolia, Plantain-leaved Cudweed(3).Cabinet.

580 Filago germanica, Herba Impia(2). - - - Cabinet.

581 Erechtites hieracifolrus, Common Fireweed(1). - - Cabinet.

582 Cacalia suaveolens, Sweetscented Indian Plantain.

583 Cacalia atriplicifolia, Indian Plantain(1). - - - Cabinet.

584. Senecio vulgaris, Common Groundsel(1). - - Cabinet.

585 Senecio atureus,

Life-root, or Squaw-weed(7). Cabinet.

586 ARNica Mollis,

Soft Arnica(2). - - - - - Cabinet. 
Latin Names.

587 Centaurea cyanus, 588 CarduUS benedictus, 589 Cirsium tanceolatum, 590 CIRSIUM DISCOLOR, 591 Cirsidm mitticum, 592 Cirsium pumilum, 593 Cirstum horridulum, 594 Chrsium arvense, 595 LAPPA MaJOR,

Vol. 20.1

596 Krigia VIRGINICA, 597 Cynthia virginica, 598 Cichorium intybus, 599 Hieracium canadense, 600 Hieracidm scabrum, 601 Hieracidm gronotir, 602 Hieracium venosum, 603 Hieracium paniculatudu, 604 Nabalus albus, 605 NabaLUS ALTISSIMUts, 606 NabalUS FRASERI, 607 Nabalus nanus, 608 Nabalus boottix, 609 Taraxacum dens-leonis, 610 Lactuca elongata, 611 Mulgedium acuminatum, 612 Mulgedium leucophedu, 613 Sonchus olkraceus, 614 Sonchus ASPER, 615 Sonchus aRVEnsis,
English Names.

Bluebottle, or French Pink(1). Cabinet. Common Blessed-thistle.

Common Thistle(1). - - Cabinet. Two-colored Thistle(2). - - Cabinet. Awnless Swamp Thistle(2). - Cabinet. Pasture Thistle(2). - - - Cabinet. Yellow Thistle(1). - - - Cabinet. Canada Thistle(2). - - Cabinet. Common Burdock(1). - - Cabinet.

Common Dwarf Dandelion(4). Cabinet. Virginian Cynthia(3). - - Cabinet. Wild Succory, or Chicory(2). Cabinet. Sharp-toothed Hawkweed(2). Cabinet. Rough Hawkweed(1). - - - Cabinet. Gronovius's Hawkweed(2). - Cabinet. Rattlesnakeweed(1). - - Cabinet. Panicled Hawkweed(2). - - Cabinet. Lionsfoot, or White Lettuce(5). Cabinet. Tall Nabalus(1). - - - Cabinet. Gall-of-the-earth.

Dwarf Nabalus(1). - - - Cabinet. Boott's Nabalus.

Common Dandetion(1). - Cabinet. Wild Lettuce, or Firewreed(4). Cabinet. Sharp-leaved Mulgredium(2). Cabinêt. Tall Mulgedium(4). - - Cabinet. Common Sowthistle(1). - - Cabinet. Spiny-leaved Sorwthistle. Large-flowered Southistle(1). Cabinet. 
Vol. 21.]

Latin Names.

616 Lobelia kalmir,

617 Lobelia nutTalin,

618 Lobelia sPicata,

619 Lobelia dortmanna,

620 Lobelia syphilitica,

621. Lobelia inflata,

622 LOBELIA CARDINALIS,
ORDER LVI. LOBELIACER.

English Names.

Kalm's Lobelia(8). - - - - Cabinet. Nuttall's Lobelia(3). - - - Cabinet. Pale-spiked Lobelia(3). - - Cabinet. Water Gladiole(3). - - - Cabinet. Blue Cardinalfower(2). - - Cabinet. Indian Tobacco(3). - - - Cabinet. Cardinalflower(3). - - - Cabinet.

ORDER LVII. CAMPANULACEA.

623 Campanula rotundifolia, Harebell(3). - - - - - Cabinet. 624 Campanula aparinoides, Slender Swamp Bellflower(3). Cabinet. 625 Campanula americana, American Bellflower(2). - - Cabinet. 626 Specularia Perfoliata, Clasping Specularia(5). - - Cabinet.

ORDER LVIII. ERICACEAE.

627 Arctostaphylos uva-Ursi, Beanberry(3). - - - - - Cabinet.

628 Clethria alnifolia,

629 Eitg-a RePENs,

630 Gadtiera procumbens,

631 ANDROMEDA POLYFOLIA,

632 ANdromeda caliyculata, Leatherleaf(3). - - - - Cabinet.

633 Andromeda mariana,

634 ANdromeda racemosa,

635 Andromeda ligustrina, Privet Andromeda(2). - - Cabinet.

636 Rhododendron maximom, Great Laurel(2). - - - - Cabinet. 637 Rhododendron lapponicum, Procumbent Alpine Rosebay(5).Cabinet. 638 Rhododendron NUdiflorum, Upright Wild Honeysuckle(4).Cabinet. 639 Rhododendron viscosum, White Wild Honeysuckle(3). Cabinet. 640 Rhododendron hispidum, Hispid Azalea.

641 Rhododendron rhodora, Rhodora(2). - - - - - Cabinet. 642 Kalmia latifolia,

643 KaLmia angustifolia, Calicotush(3). - - - Cabinet.

Common Sweєt Pepperbush(3). Cabinet. Ground Laurel(4). - - - - Cabinet. Partridgeberry, or Teaberry(6).Cabinet. Kill-lamb, or Staggerbush(3). Cabinet. Racemed Andromeda(2). - - Cabinet. Dwarf or Sheep Laurel(2). - Cabinet. 
Latin Names.

644 KaLmia GLaDCa,

645 Ledur palustre,

646 Vaccinidn ULIGINosUM,

647 Vaccinium vacillans,
English Names.

Swamp Laurel(4). - - - - Cabinet.

Labrador-tea $(3)$. - - - Cabinet.

Alpine Bilberry(1). - - Cabinet.

Low Blue Huckleberry(1). - Cabinet.

648 Vaccinium pennsylvanicur, Dwarf Blue Huckleberry(3). Cabinet.

649 Vacciniom corymbosum, Tall Swamp Huckleberry(5). Cabinet. 650 Vaccinium canadense, Black Bilberry(1). - - Cabinet. 651 Vaccinium staminedr, Deerberry, or Squaw Huckleberry(3).Cabinet. 652 VACcinium oxycoccus, Small Cranberry(2). - - Cabinet. 653 Vaccinium macrocarpon, Common Cranberry(5). - - Cabinet. 654 GaylussaCia hirtella, 655 Gaylunsacia frondosa, 656 Gaylussacia resinosa, 657 Chiogenes hisptdula, 658 Pyrola rotundifolia,

659 Pyrola elliptica, 660 Pyrola chlorantha, 661 Pyrola uliginosa, 662 Pyrola secunda, 663 Pyrola uniflora, 664 Chimaphila ombellata, 665 Chimaphila maculata, 666 MoNotropa tNIFLoRa, Dwarf Swamp Huckleberry.

Bluetangle, or Dangleberry(1). Cabinet. Black Huckleberry(2). - - Cabinet. Creeping Snowberry(2). - - Cabinet. Round-leaved Wintergreen(3). Cabinet. Shinleaf(3). - - - . - Cabinet. Greenish-flowered Wintergreen(2). Cabinet. Swamp Wintergreen.

Onesided Wintergreen(4). - Cabinet. One-flowered Wintergreen(6). Cabinet. Pipsissewa, or Prince's-pine(4). Cabinet. Spotted Wintergreen(4). - - Cabinet. Indian-pipe(6). - - - - Cabinet. 667 Monotropa lanuginosa, Pinesap, or False Beechdrops(7). Cabinet. 668 Pterospora andromedea, Giant Birdsnest(2). - - - Cabinet.

Vol. 22.]

669 ILEX OPACA,

670 Ilex ambiguUs,

671 Prinos verticillatus, "

672 Prinos levigatus,
ORDER LIX. AQUIFOLIACE丑. American Holly(3). - - Cabinet. Ambiguous Ilex(2). - - Cabinet. Common Winterberry(3). - Cabinet. Smooth Winterberry(2). - - Cabinet. 673 Prinos glaber, Evergreen Winterberry, or Inkberry(2). Cabinet. 674 Nemopanthes canadensis, Mountain Holly(2). - - Cabinet. 
ORDER LX. EBENACEA.

Latin Names.

English Names.

675 Diospyros virginiana, Persimmon(3). - - - - - Cabinet.

ORDER LXI. PRIMULACEA.

676 Primula mistassinica,

677 Lysmachia stricta,

678 Lysimachia qUadrifolia,

679 Inysimachia ciliata,

680 Lisimachia hybrida,

681. Lysimachia longifolia,

682 Lysimachia THYRSIFLORA,

683 Trientalis americana,

684 ANagallis arvensis,

685 Hottonia inflata,

686 Samolus Valerand,
Dwarf Canadian Primrose(6). Cabinet.

Racemed Locsestrife(2). - - Cabinet.

Whorled Loosestrife(3). - - Cabinet.

Fringed Loosestrife(2). - - Cabinet.

Hybrid Loosestrife(1). - - Cabinet.

Revolute Loosestrife(1). - - Cabinet.

Tufted Loosestrife(3). - - - Cabinet.

Chickweed Wintergreen(4). - Cabinet.

Scarlet Pimpernel(4). - - - Cabinet.

American Waterfeather(1). - Cabinet.

Common Water Pimpernel(3). Cabinet.

ORDER LXII. PLANTAGINACE

687 Plantago major,

688 Plantago cordata,

689 Plantago lanceolata,

690 Plantago virginica,

691 Piantago maritima,

692 Plantago pusilla,
Broad-leaved Plantain( $\mathbf{1})$. - Cabinet.

Heart-leaved Plintain(2). - Cabinet. Ribgrass(2). - - - - - Cabinet. White Plantain(4). - - - Cabinet. Sea Plantain(4). - - - - Cabinet. Dwarf Plantain(10). - - - Cabinet.

ORDER LXIII. PLUMBAGINACEA.

693 STatice LIMONIUM,

Common Marsh Rosemary(1). Cabinet.

ORDER LXIV. LENTIBULACE 坐.

694 Pinguicula volgaris,

695 UTRICULARIA INFLATA,

696 UTRICULARIA PURPUREA,

697 UTRICULARIA VULgaris,

698 UTRICULARIA CORNUTA,

699 Utricularia striata,

700 UTRICULARIA INTERMEDIA,

701 UTRICULARIA MINOR,
Common Butterwort(3). - - Cabinet. Spongy-leaved Bladderwort(2). Cabinet. Purple Bladderwort.

Common Bladderwort(3). - Cabinet.

Sharp-horned Bladderwort(5). Cabinet.

Striated Bladderwort.

Intermediate Bladderwort,

Lesser Bladderwort. 
ORDER LXV. OROBANCHACEA.

Latin Names.

702 Orobanche americana,

703 Orobanche uniflora,

704 Epiphegus americana,
English Names.

Squauroot(2). - - - - Cabinet.

Long-stalked Broomrape(4). Cabinet. Beechdrops, or Cancer-root(3). Cabinet.

Vol. 23.]

ORDER LXVI. BIGNONIACE $\boldsymbol{A}$.

705 Catalia syringerfolia, Indian Bean.

ORDER LXVII. PEDALIACEA.

706 Martynia Pfoboscidea, Unicorn-plant(2). - - - Cabinet.

ORDER LXVIII. ACANTHACEA.

707 Dianthera americana, Water Willow(2. - - - - Cabinet.

ORDER LXIX. SCROPHULARIACE AE.

708 Verbascum thapsus,

709 VERBascum bLATTARIA,

710 Vergascum Lychitis,

711 Scrophularia marilandica, Figwort(2). - - - - - Cabinet.

712 Linaria elatine,

713 LiNaria vULGaris,

714 Linaria canadensis,

715 Collinsia verna,

716 Chelone glabra,

717 Pentstemon pubescens,

718 Mimulus Ringens,

719 Mimulus alatus,

720 Gratiola virginica,

721 Gratiola aUREA,

722 LiNdERNia Dilatata,

723 Lindernia attenuata,

724 BUChNERA AMERICANA,

725 Limoselda AQUaTICA,

726 VERONICA SERPYLLIFOLIA, 727 VERONICA OFFICINALIS,
Common Mullein(2). - - Cabinet.

Moth Mullein(2). - - - - Cabinet.

White Mullein(4). - - - - Cabinet.

Hairy Toadflax.

Common Toadflax(3). - - - Cabinet.

Canadian Toadflax(4). - - Cabinet.

May-beauty(5). - - - - - Cabinet.

Shellflower, or Snakehead(2). Cabinet.

Pubescent Pentstemon(1). - Cabinet.

Common Monkeyflower(2). - Cahinet.

Wing-stemmed Monkeyflower(2). Cabinet.

Common Hedgehyssop (3). - Cabinet.

Golden Hedgehyssop(4). - - Cabinet.

Long-stalked Lindernia(3). - Cabinet.

Short-stalked Lindernia(3). - Cabinet. Bluehearts(4). - . - - Cabinet.

Common Mudwort(5). - - Cabinet.

Paul's Betony(7). - - - - Cabinet.

Common Speeduell. 
Latin Names.

728 VERONICA BECCABUNGA,

729 VERONICA aNAGaLLIS,

730 Veronica scutellata,

731 VERONICA PEREGRINA,

732 Veronica arvensis,

733 Veronica agrestis,

734 VERONICA HEDERIFOLIA,

735 P rederota virginica,

736 Gerardia tenudfolia,

737 Gerardia pURPUREa,

738 Gerardia maritima,

739 Gerardia pedicularia,

740 Gerardia flava,

741 Gerardia quercifolia,
English Names.

American Brooklime(2). - - Cabinet.

Water Speedwell(1). - - - Cabinet.

Marsh Speedwell(3). - - - Cabinet.

Neckweed.

Corn Speedwell(5). - - - Cabinet.

Field Speedwell(1), - - - Cabinet.

Ivy-leaved Speedwell(8). - - Cabinet. Culver's-physic(5). - - - - Cabinet.

Slender-stalked Gerardia(4). Cabinet.

Rough-leaved Gerardia(2). - Cabinet.

Saltmarsh Gerardia(2). - - Cabinet.

Bushy Gerardia(3). - - - Cabinet.

Pubescent False-foxglove(3). Cabinet.

Glaucous False-foxglove(3), - Cabinet.

742 Castilleja coccinea, Scarlet Paintedcup, or Redrobin(3).Cabinet.

743 Pedicularis canadensis, Common Lousewort(4). - - Cabinet.

744 Pedicularis lanceolata, Tall Lousewort(2). * - - Cabinet.

745 Melampinum americanum, American Cow-wheat(3). - - Cabinet.

Vol. 24.]

746 VERBENa hastata,

747 VERBENA URTICIFOLIA,

748 VERBENA sPuRIa,

749 Verbena aigústifolia,

750 Phryma leptostachya,
ORDER LXX. VERBENACEA.

Tall Blue Vervain(3). - - Cabinet.

Common Vervain(3). - - Cabinet.

Procumbent Vervain.

Narrow-leaved Vervain(2). - Cabinet.

Lopseed(2). - . - - - - Cabinet.

ORDER LXXI. LABIAT $Æ$.

751 Isanthus cerruleus,

752 MeNTha viRIDIs,

753 Mentha piperita,

754 Mentha canadensis,

755 Lycofus sinuatus,

756 LYycopUs VIRGINICUS,

757 Monarda pIDYMa,
False Pennyroyal(1). - - - Cabinet.

Spearmint(1). - - - - C Cabinet.

Peppermint(2). - - - - Cabinet.

Canadian Mint(2). - - - Cabinet.

Common Water Horehound(2). Cabinet. Bugleweed(1). - - - - Cabinet. Oswego-tea(2). - . - . - Cabipet, 
Latin Names.

758 Monarda fistulosa,

759 Monarda punctata,

760 Blephilia hiRsuta,
English Names.

Wild Bergamot(1). - - - Cabinet.

Horsemint.

Hairy Blephilia(2). - - - Cabinet.

Common Mountain-mint(2). - Cabinet.

762 Pycnanthemum clinopodioides, Basil-leaved Mountain-mint(1).Cab. 763 Pychanthemum torrei, Torrey's Mountain-mint(2). - Cabinet. 764. Prcnanthemum muticum, Broad-leaved Mountain-mint(2).Cabinet. 765 Pycnanthemum lanceolatum, Virginian Thyme(2). - Cabinet. 766 Prcnanthemum linifolium, Narrow-leaved Thyme(1). - Cabinet. 767 ORIGANUM VULGaRE, Wild Marjoram(2). - - - Cabinet. 768 Collinsonia canadensis, Common Horsebalm(2). - - Cabinet. 769 Cunila mariana, Common Dittany(3). - - - Cabinet.

770 Hedeoma pulegioddes,

771 Mickomeria glabella, 772 Melissa clinopodium, 773 Melissa officinalis, 774 Prunella vulgaris, 775 Scttellaria pilosa, Pennyroyal(3). - - - Cabinet.

Niagara Thyme(5). - - C Cabinet. Wild Basil(3). - - - Cabinet. Common Balm(1). - - - Cabinet. Common Selfheal, or Healall(3). Cabinet. Hairy Scullcap(2). - - Cabinet. 776 Scutellaria integrifolia, Entire-leaved Scullcap(2). - Cahinet. 777 Scutellaria parvila, 778 Scutellaria Nervosa, Small Scullcap(4). - - - Cabinet. Nerved Scullcap.

779 Scttellaria galericulata, Common Scullcap(2). - - Cabinet. 780 Scuteldaria lateriflora, Mad-dog Soulloap(1). - - - Cabinet. 781 Lophanthus nepetoides, Yellow Giant-hyssop(1). - - Cabinet. 782 Lophanthus scropholarimfolius, Purple Giant.hyssop(2). Cabinet. 783 Nepeta cataria, Common Catnep(2). - - - Cabinet. 784 Nepeta gLechoma, Groundivy, or Gill(2). - - Cabinet.

785 Dracocephalum Parviflorum, Small-flowered Dragonshead(2).Cab. 786 Physostegia virginiana, Virginian Dragonshead(3). - Cabinet. 787 Lamitm a mplexicadde, Common Deadnettle, or Henbit(2). Cabinet. 788 LeONuRa cardiaca, Common Motherwort(2). - - Cabinet. 789 Markubium volgare, Common Horehound(2). - - Cabinet. 
Latin Names.

790 GaLkOPSIS TETRAhIT,

791 STACHYS ASPERA,

792 STachys PaLUSTRIs,

793 Stachys hyssopifolua,

English Names.

Common Hempnettle(2). - - Cabinet. Rough Hedgenettle(1). - - Cabinet.

Marsh Hedgenettle(1). - - Cabinet.

Narrow-leaved Hedgenettle(2). Cabinet. 794 Trichostema dichotoma, Bluecurls, or False Penxyroyal(2).Cabinet. 795 Teucriom canadense, Woodsage, or Germander(2). Cabinet.

Vol. 25.]

796 ONosmodium HisPIDUM,

797 Echivm volgare,

798 Pulmonaria virginica,

799 Lithospermura ARVENSE,

\section{ORDER LXXII. BORAGINACEA.}

False Gromwell(2). - - - - Cabinet. Viper's Bugloss(2). - - - Cabinet. Virginian Lungwort(2). - - Cabinet. Corn Gromwell, or Stoneweed(2).Cabinet. 800 Lithospermum officinale, Common Gromwell.

801 Batschia canescens, Common Puccoon(2.. - - - Cabinet. 802 Lycopsis arvensis, 803 Myosotis palustris, 804 Mrosotis arvensis, Small Bugloss(1). - - - - Cabinet. American Forget-me-not(5). - Cabinet. Field Scorpion-grass(5). - - Cabinet. 805 Srmphitum officinale, Common Comfrey(1). - - Cabinet. 806 Cxnoglossum officinale, Common Houndstongue(2). - Cabinet. 807 Cynoglossum virginicum, Wild Comfrey(2). - - - Cabinet. 808 Echinospermum lappula, Narrow-leaved Stickseed(2). - Cabinet. 809 Echinospermum virginicum, Broad-leaved Stickseed(2). - Cabinet.

\section{ORDER LXXIIT. HYDROPHYLLACEAE.}

810 Hydrophyllum virginicum, Virginian Waterleaf(3). - Cabinet. 811 Hydrophylud canadense, Canadian Waterleaf $(2)$. - Cabinet. 812 Hydrophyllum appendtoulatum, Hairy Waterleaf(1). - Cabinet.

ORDER LXXIV. POLEMONIACEÆ.

813 Phlox divaricata,

814 Phzox subulata,

815 Polemonium reptans,
Divaricate Phlox(1). - - - Cabinet. Mountain Pink(1). - - - Cabinet. Jacob's-Zadder(1). - - - - Cabinet.

ORDER LXXV. DLAPENSIACEAE.

816 Diapensia lapponica, Lapland Diapensia(7). - - Cabinet. 
Vol. 26.] ORDER LXXVI. CONVOLVULACEAE.

Latin Names.

Englısh Names.

817 Convolvulus arvensis, Common Bindweed(2). - - Cabinet.

818 Convolvulus panduratus, Man-of-the-earth(4). - - Cabinet.

819 Calystegia sepium, Great Bindweed(6). - - Cabinet.

820 Calystegia spithamea, Upright Bindweed(3). - - Cabinet.

821 Cuscuta gronovir, Common Dodder(4). - - - Cabinet.

822 Cuscuta umerosa,

823 Cúscuta epilinum,

Smooth-flowered Dodder.

Flax Dodder(1). . - - = Cabinet.

ORDER LXXVII. SOLANACEAS.

824 Nicotlana rustica,

825 Datura stramonium,

826 Hyoscyamus niger,

827 NicandRa PHysaloides,

828 Physalis viscosa,

829 Solanum dulcamara,

830 Solandm Nigrum,

831 Solanum Carolinianum,

Wild Tobacco(2). - - - Cabinet. Jamestown-weed, or Stinkweed(1).Cabinet. Common Henbane(2). - - Cabinet. Apple-of-Peru(2). - - - Cabinet. Groundcherry(3). - - - Cabinet. Bittersweet(2). - - - - Cabinet. Common Nightshadt)2). - - Cabinet. Horsenettle.

\section{ORDER LXXVIII. GENTIANACE}

832 Gentiana saponaria, 833 Gentiana andrewsit, 834 Gentiana ochroleuca, 835 Gentiana quinqueflora, Five-flowered Gentian(1). - Cabinet. 836 Gentiana detonsa, 837 Gentiana grinita, 838 Frasera carolinensis, 839 Halenta deflexa, 840 ERythrea centaURiUm, 841 ERythrea MUHLENBERgII, 842 Bartonia tenella, 843 Sabbatia stellaris, 844 Sabbatia angularis,
Soap Gentian(2). - - - - Cabinet. Andrews's Gentian(7). - - Cabinet. Ochroleucous Gentian.

Smaller-fringed Gentian(2). Cabinet. Large-fringed Gentian(3). - Cabinet. American Columbo(3). - - Cabinet. Deflexed Halenia(1). - - - Cabinet. Common Centaury(1). - - - Cabinet. Muhlenberg's Centaury(1). - Cabinet. Late-flowered Bartonia(5). - Cabinet. Saltmarsh Centaury(7). - - Cabinet. American Centaury(3). - - Cabinet. 
Latın Names.

845 Sabbatia chloroides,

846 Menyanthes trifoliat

847 Limnanthemum lacunosum, Floatingheart(2). - - - Cabinet.

Vol. 27.]

ORDER LXXIX، APOCYNACEAE.

848 A pocynum andros emifolium, Dogsbane(5). - - - Cabinet.

849 Afocynum cannabinum, Indian $\operatorname{Hemp}(2)$. - - - Cabinet.

\section{ORDER LXXX. ASCLEPIADACE正}

850 Aschepias CORnuti, Silkweed, or Milkweed(2). - Cabinet.

851 Asclepias purpurascens, Purple Silkweed(5). - - Cabinet.

852 Asclefpias phy tolaccoides, Poke-leaved Milkweed(3). - Cabinet.

853 Ascleptas obtusifolia, Waved-leaved Milkweed(2). - Cabinet. 854 Asclepias variegata, Variegated Silkweed(2). - - Cabinet. 855 Asclefias quadrifolia, Four-leaved Silkweed(2). - Cabinet. 856 Asclepias incarnata, Swamp Silkweed(2). - - Cabinet. 857 Asclepias tuberosa, Pleurisy-root, or Butterfy-weed(y). Cabinet. 858 Asclepias verticillata, Whorled Silkweed(3). - - - Cabinet. 859 Acerates viridiflora, Green-flowered Silkweed(3). - Cabinet.

\section{ORDER LXXXI. OLEACEA.}

860 Fraxinus americana, White Ash(5). - - - - Cabinet. 861 Fraxinus sambucifolia, Black Ash, or Water Ash(1). Cabinet. 862 Fraxinus PUBESCENS, 863 Ligustrum vulgare, Gray $A s h(1)$. - . - - Cabinet. Common Privct(2). - - Cabinet.

\section{Vol. 28.] ORDER LXXXII. ARISTOLOCHIACEA.}

864 Aristolochia serpentaria, Virginia Snakeroot(2). - - Cabinet. 865 Asarum canadense, Wild Ginger, or Coltsfoot(2). Cabinet.

ORDER LXXXIII. CHENOPODIACEA.

866 Chenopodium alibum,

867 Chenopodium hybridum,

868 Anbrina botrys,

869 Ambrina anthelmintica,
Goosefoot, or Laml's-quarters(2).Cabinet. Maple-leaved Goosefoot(3). - Cabinet. Jerusalem-oak(2). - - Cabinet. Wormseed(1). - - - - Cabinet. 
Latin Names.

870 Ambrina ambrosiotdes,

871 Blitum Maritimum,

872 Blitum capitatum,

873 Blitum BONtJ-HENRicus,

874 Atriplex patula,

875 Odione arenaria,

876 Acnida cannabina,

877 Acnida rusocarpa,

878 SALICORNIA HERRACEA,

879 Salicornia mucronata,

880 Salicornta ambigua,

881 Sueda maritima,

88? Salsola kali,
English Nanes.

Mexican Tea(1). - - - Cabinet.

Saltmarsh Blite.

Strauberry Blite(1). - - - Cabinet.

Good-King-Henry(2). - - C Cabinet.

Halbert-leaved Orach(3). - - Cabinet.

Seabeach $\operatorname{Orach}(\mathbf{1}) . \quad$ - - Cabinet.

Common Waterhemp(1). - - Cabinet.

Rough-fruited Waterhemp (1). Cabinet.

Cammon Saltwort, or Samphire(2). Cabinet.

Dwarf Saltwort(6). - - Cabinet.

Perennial Saltwort(1). - - Cabinet.

Smooth Glasswort(1). - - - Cabinet.

Common Glasswort(1). - - Cabinet.

\section{ORDER LXXXIV. AMARANTHACEA.}

883 Amaranthus hybridus, Hybrid Amaranth(1). - - Cabinet.

884 Amaranthus gracizans, Bushy Amaranth(1). - - Cabinet. 885 Amaranthus pumilus, Dwarf Amaranth(1). - - Cabinet.

886 Amaranthus deflexus, Prostrate Amaranth(1). - Cabinet.

Vol. 29.] ORDER LXXXV. POLYGONAOE正.

887 Polygonum orientale, Princesfeather $(1)$. - - - Cabinet. 888 Polygonum fagopyrum, Buckwheat(2). - - - - Cabinet. 889 Polygonum convolvulus, Black Bindweed(1). - - - Cabinet. 890 Polygonum cilinode, Fringe-jointed Polygonum(1). Cabinet. 891 Polygonum dumetorum, Climbing Buckwheat(1). - - Cabinet. 892 Polygonum sagitratum, Arrow-leaved Tearthumb(2). Cabinet. 893 Polygonum arifolium, Halbert-leaved Tearthumb(2). Cabinet. 894 Polygonum a.mphibium, Water Persicaria(2). - - Cabinet. 895 Polygonum persicaria, Ladysthumb(3). - - - Cabinet. 896 Polygonum pennsylvanicum, Hairy-stalked Persicaria(2). Cabinet. 897 Polygonum Mite, Bearded Persicaria(3). - - Cabinet. 898 Polygonum hydropiper, Water Pepper, or Smartweed(3).Cabinet. 899 Polygonom virginianom, Virgate Persicaria(1). - - Cabinet. 
plants.

Latin Names.

English Names.

900 Polygonum aviculare, Knotgrass, or Doorweed(3). - Cabinet.

901 Polygonum Maritimum, Seaside Knotgrass(2), - - - Cabinet. 902 Polygonum tenue,

Slender Knotgrass(4). - - Cabinet.

903 Polygonum articulatum, Jointweed(5). - - - - - Cabinet.

904 RuMEX cRIsPus, Curled Dock(1). - - - - Cabinet.

905 RUMEX OBTUSIFOLIUS, Broad-leaved Dock(2). - - Cabinet.

906 Rumex verticillatus, Long-stalked Waterdock(1). - Cabinet. 907 Rumex britannica, 908 Rumex acetosella,

Yellow-rooted Waterdock(2). Cabinet. Sheepsorrel(3). - - - - Cabinet.

ORDER LXXXVI, PHYTOLACCACE $Æ$.

909 Phytolacca decandra, Common Pokeweed(3). - - Cubinet.

ORDER LXXXVII. LAURACEA.

910 Sassafras officinale, Sassafras(4). - - - - Cabinet.

911 Benzoin odoriferum, Wild Alspice, or Spicebush(4). Cabinet.

ORDER LXXXVIII. SANTALACEAS.

912 Comandra umbellata, Bastard Toadflax(3). - - - Cabinet.

913 Nyssa multiflora, Blackgum(6). - - - - Cabinet.

ORDER LXXXIX. THYMELACE王.

914 Dirca palustris,

Leatherwood(4). - - - - Cabinet.

ORDER XC. ELEAGNACE A.

915 Shepherdia canadensis, Canadian Shepherdia(2). - Cabinet.

ORDER XCI. ULMACEAE.

916 Ulmos AMERICANA,

917 Ulmus FULVA,

918 ULMUS KACEMOSA,

919 Celtis occidentalis,
American or White Elm(4). - Cabinet. Slippery or Red Elm(4). - - Cabinet. Thomas's Elm(3). - - - - Cabinet. Netlletree, or Sugarberry(3). Cabinet.

Vol. 30.]

ORDER XCII. SAURURACE $\mathrm{E}$.

920 SaUrurus cernuds,

Lizardstail(1). - - . - . Cabinet.

ORDER XCIII. CERATOPHYLLACE $\$$.

921 Ceratophyldom echinatum, Rough-fruited Hornuort(3). Cabinet, 


\section{ORDER XCIV, CALLITRICHACEAE.}

Latin Names.

922 Callitriche verna,
English Names.

Common Water Chickweed(7). Cabinet.

ORDER XCV. PODOSTEMACE $Z$.

923 Podostemum ceratophyllom, Riverweed(6). - - - - Cabinet.

ORDER XCVI. EUPHORBIACEA.

924 Acalyfha virginica, Common Three-seeded Mercury(5).Cabinet. 925 Euphorbia helitoscoyia, Waterwort Spurge(3). - - Cabinet. 926 Euphorbia platyphylla, Broad-leaved Spurge.

927 Euphorbia corollata, Flowering Spurge(2). - - Cabinet. 928 Euphorbia hypericifolia, Upright Spotted Spurge(3), Cabinet. 929 Euphorbia maculata, Small Spurge(2). - - - - Cabinet. 930 Euphorbia ipecactuanha, Wild Ipecac(2). - - - - Cabinet. 931 Euphorbia polygonifolia, Seaside Spurge(2). - - - Cabinet.

\section{ORDER XCVII. EMPETRACEA.}

932 Emṕtrum NigRum,

Common Crowberry(2). - - Cabinet.

\section{ORDER XCVIII. JUGLANDACEA.}

933 . Juglans Nigra,

934 Juglans cinerea,

935 Carya alba,

936 Carya tomentosa,

937 Carya gLabra,

938 Carya amara,
Black Walnut(1). - - - - Cabinet.

Butternut(1). - - , - Cabinet.

Shellbark Hickory.

White-heart Hickory(2). - - Cabinet.

Broom Hickory, or Pignut(2). Cabinet. Swamp Hickory, or Bitternut(1). Cabinet.
Vol. 31.]

939 Ostrya virginica,

940 Carpinus americana,

941 Corylus americana,

942 Corylus rostrata,

943 Quercus phellos,

944 Quercus nigra,

945 Quencus tinctoria,
ORDER XCIX. CUPULIFER $\approx$,

Hop Hornbeam, or Ironwood(5). Cabinet.

Water Beech, or Hornbeam(3). Cabinet. American Hazlenut(2). - - Cabinet. Beaked Hazlenut(1). - - - Cabinet. Peach-leaved or Willow Oak(2).Cabinet. Black-jack Oak(5). - - - Cahinet. Black Oak, or Quercitron(1). Cabinet. 
PLANTS,

Latin Names.

946 QJercus COCCINea,

947 Quercts rubra,

948 QUERCUS PaLUSTRIs,

949 Quercus ILICIFolIA,

950 Quercus obtusiloba,

951 Querous macrogarpa,

952 QuERCUS OLIV TFFormis,

953 Quercus alba,

954 QUERCUS BICOLOR,

955 Quercus montana,

956 Quercus castanea,

957 Quercus PRINOIDEs,

958 FaGUS FERRUGinea,

959 Castanea vesca,

960 Castanea pumila,

English Names.

Scarlet Oak(4). - - - Cabinet.

Red $\operatorname{Oak}(2)$. - - . - Cabinet.

Pin Oak, or Water Spanish Oak(2).Cabinet. Barren Scrub Oak(3). - - Cabinet. Post Oak, or Box White Oak(3).Cabinet. Overcup White Oak(3). - - Cabinet. Mossycup Ogk,

White $\operatorname{Oak}(2)$. - - - Cabinet. Swamp White Oak(1). - - Cabinet. Rock Chesnut Oaki(1). - Cabinet. Yellow or Chesnut Oak(1). - Cabinet. Chinquapin Oak(2). - - Cabinet. Beech(5). - - - - Cabinet. American Chestnut(1). - Cabinet. Chinquapin.

ORDER C. MYRICACEA.

961 Myrica GaLe, Dutch Myrtle, or Sweet Gale(3) Cabinet. 962 MyrtCA GERIFERA, 963 Comptonia Asplenifolia, Sweet Fern(4). - - - - Cabinet.

\section{ORDER OI. BETULACE E.}

964 Betula populifolia,

965 Betdula paptracea,

966 Betula lenta,

967 Betula excelsa,

968 BETULA NIGRA,

969 Betula nana,

970 Alnus serrdlata,

971 Amnos incana,

972 Alnus viridis,
White Birch(2). - - - Cabinet.

Paper or Canoe Birch.

Black or Sweet Birch(3). - Cabinet, Yellow Birch(2). - - - - Cabinet. Red Birch(1). - - - - Cabinet. Dwarf Birch(1). - - - Cabinet. Common Alder(2). - - - Cabinet. Black Alder(1). - - - Cabinet. Mountain Alder(2). - - Cabinet.
Vol. 32.]

973 SALIX CANDIDA,
ORDER CII. SALICACEA. 
Latin Names.

974 Salix MuHLENbERgiana,

975 SALIX TRISTIS,

976 Salix miscolor,

977 Salix PETIOLARIS,

978 SALIX MYRICOIDES,

979 Salix viminalis,

980 SaLIX LUCIDA,

981 Salix NIGRA,

Vol. 33.]

982 SALIX LONGIFOLIA,

983 Salix vitellina,

984 Salix rostrata,

985 SALIX CORDATA,

986 Salix RIgida,

987 SALIX PEDICELLARIS,

988 SAlix cUtLERI,

989 Populus treiduloides,

991 Populus monilifera,

992 Populus heter ophylla,

993 Popdlus nigra,

994 Populus balsamirera, Balsam Poplar, or Tacamahac(1).Cabinet. 995 Populus candicans,

English Names.

Muhlenberg's Willow(5). - Cabinet. Dwarf Downy Willow(4). - Cabinet. Glaucous Willow(13). - - Cabinet. Dark Long-leaved Willow(7). Cabinet. Gale-leaved Willow.

Basket Osier(2). - - - Cabinet. Glossy Broad-leaved Willow(9). Cabinet. Black Willow(9). - - - - Cabinet,

Long-leaved Sand Willow(1). Cabinet. Yellow Willow(1). - - - - Cabinet. Ochre-flowered Willow (17). $\quad$ Cabinet. Heart-leaved Willow(9). - - Cabinet. Rigid Heart-leaved Willow(4). Cabinet. Long-stalked Willow(2). - - Cabinet. Cutler's Willow, or Bearberry(3).Cabinet. American Aspen(2). - - - Cabinet. Virginian Poplar(8), - - Cabinet. Cotton-tree(2). - - - - Cabinet. Birch-leaved Poplar. Balm-of-Gilead Poplar(2). - Cabinet.

Vol. 34.] ORDER CIII. BALSAMIFLUÆE.

996 Liquidambar styraciflua, Common Sweetgum(3). - - Cabinet.

ORDER CIV. PLATANACEA.

997 Platanus occidentalis, Buttorwood, or Sycamore(2). Cabinet.

ORDER CV. URTICACE

998 MORUS RUBRA, Red M(ulberry(1). - - - Cabinet. 999 Morus alba, 1000 Urtica DIoICA, White Mulberry(2). - - - Cabinet. Common Stinging Nettle. 
Latin Names.

1001 URtica UReNS,

1002 URTica CANADENSis,

1003 Adike FUMILA,

1004 Begumeria cylindrica,
English Names.

Small Stinging Nettle(2). - Cabinet.

Canadian Nettle(2). - - - Cabinet.

Richweed, or Clearweed(8). - Cabinet.

False Nettle(2). - - - - Cabinet.

1005 Parietaria pennsylvanicum, Pennsylvanian Pellitory(4).Cabinet. 1006 Cannabis sativa, Common Hemp(2). - - - Cabinet. 1007 Humulus lupulus, Hop(1). - - - - - Cabinet.

\section{CLASS II. GYMNOSPERMOUS PLANTS.}

Vol. 35.]

1008 Pinus Resinosa,

1009 Pinus Rigida,

1010 Pinus strobus,

1011 Pinus mitis,

1012 Pinus balsamiea,

1013 Pinus canadensis,

1014 Pinus nigra,

1015 PinUS ALBA,

1016 PiNUS PENDULA,

1017 Cupressus thuyoIDEs,

1018 Thuya occidentalis,

1019 JUNiperus CONMUNIS,

1020 Juniperus virginiana,

1021 Taxus Canadensis,
ORDER CV. CONIFERA.

Red Pine.

Pitch Pine(2). - - - - Cabinet.

White Pine(1). - - - - - Cabinet.

Yellow Pine.

Balsam Fir, or Balm-of-Gilead Fir.

Hemlock Spruce(2). - - Cabinet.

Black or Double Spruce.

White or Single Spruce.

American Larch, or Tamarack(2).Cabinet. White Cedar(3). - - - - Cabinet.

Common Arbor-vite(3). - - Cabinet.

Common Juniper(3). - - - Cabinet. Red Cedar(4). - - - - Cabinet. American Year(1). - - - Cabinet.

\section{CLASS III. ENDOGENOUS PLANTS.}

Vol. 36.]

1022 ARIS RMA TRIPHYLIA,

1023 Arisema dracontidm,

1024 Peltandra virginica,

1025 Cadia palustris,

\section{ORDER CVI. ARACEX.}

Indian Turnip(2). - - - Cabinet. Greendragon(1). - - - Cabinet. Arrow-leaved Arum(2). Cabinet. Water $\operatorname{Arum}(\mathbf{1})$. - - - Cabinet. 
Latin Names.

1026 Symplncarpus føtidus, Common Skunk-cabbage(4). - Cabinet.

1027 Orontium aquaticum,

1028 acorus calamus,
Goldenclub(4). - - - Cabinet.

Common Calamus, or Sweetflag(2).Cabinet.

ORDER CVII. LEMNACEA.

1029 LEMNA MINOR,

1030 Lemina perpusilla,

1031 Lemana trisulca,

1032 Lemna gibba,

1033 lemana polyrrhiza,
Lesser Duckweed(3). - - - Cabinet.

Smallest Duckweed(1). - - Cabinet.

Star Duckweed(2). - - - Cabinet.

Gilbous Duckweed.

Greater Duckweed(2). - - Cabinet.

ORDER CVIII. TYPHACEAE.

1034 Typha latifolia,

1035 Typha angustifolia,

1036 Sparganidi ramostm,

1037 Sparganidm simplex,
Broad-leaved Cattail(2). - - Cabinet. Narrow-leaved Cattail(1). - Cabinet.

Branching Bur-reed(2). - - Cabinet. Smaller Bur-reed(4). - - - Cabinet.

ORDER CIX. NAJADACERE.

1038 Najas canadensis,

1039 Zostera MIARINA,

1040 Ruppia maritima,

Canadian Waternymph(3). - Cabinet. Common Grasswrack(2). - - Cabinet. Ditchgrass(1). - - - Cabinet. 1041 Zannichellita palustris, Horned Pondweed(2). - - Cabinet. 1042 Potamogeton natans, Broad-leaved Floating Pondweed(5). Cab. 1043 Potamogeton heterophyluds, Various-leaved Pondweed(1). Cab. 1044 Potamogeton hybrinds, Small Floating Pondweed(3). Cabinet. 1045 Potamogeton lucens, Shining Pondweed(3). - Cabinet. 1046 Potamogeton perfoliatus, Perfoliate Pondweed(1). - Cabinet. 1047 Potamogeton zosterifolius, Grass-leaved Pondweed(6). Cabinet. 1048 Potamogeton pusillus, Small Pondweed(3). - - - Cabinet. 1049 Potamogeton Pauciflorus, Few-flowered Pondweed(4). Cabinet. 1050 Potamogeton pectinatus, Fennel-leaved Pondweed(1). - Cabinet.

\section{ORDER CX. ALISMACEAE.}

1051 Alisma plantago, Common Water Plantain(2). Cabinet. 1052 Sagittaria sagitrifolia, Common Arrowhead(14). - - Cabinet. 
Latin Names.

1053 Sagittaria pusillaA,

1054 Triglochin palustre,

1055 Triglochin maritimdu,

1056 Triglochin elatum,
English Names

Least Arrowhead.

Marsh Arrowgrass(3). - - Cabinet.

Seaside Arrowgrass(4). - - Cabinet.

Tall Arrougrass(1). - - - Cabinet. - Cabinet.

ORDER CXI. HYDROCHARIDACEEE.

1058 Hydrocharis cor Difolia, Heart-leaved Frogsbit.

1059 UdORA GANADENSIS, 1060 Valisneria spiralis,
Little Watersnakeweed(4). - Cabinet. Tapegrass, or Eelgrass.

Vol. 37.]

ORDER CXII. ORCHIDACEA.

1061 Microstyuls monopiryllos, Short-stalked Addersmouth(2). Cabinet. 1062 Microstylis ophioglossoides, Long-stalked Addersmouth(2). Cab. 1063 Coraldorrhiza innata, Vernál Coralroot(3). - - - Cabinet. 1064 Corallorrhiza odontorraiza, Small Late Coralroot(4). Cabinet. 1065 Corallorrhiza Multiflora, Large Coralroot(5). - - Cabinet. 1066 Aplectrum hyemale, Puttyroot, or Adam-and-Eve(2).Cabinet. 1067 Lifaris lititfolia, Common Twayblade(2). - - Cabinet. 1068 LITPARIS LAESELII, 1069 Tiputaria discolor, 1070 OrChIS SPECTABILIS, Smaller Twayblade(2). - - Cabinet. Cranefly Orchis(2). - - - Cabinet. Showy Orchis(2). - - - Cabinet. 1071 Gymidania tridentata, Three-toothed Gymnadenia(2). Cabinet. 1072 Platanthera obtusata, Obtuse-leaved Platanthera(1). Cabinet. 1073 Platanthera orbiculata, Large-leaved Orchis(1). - - Cabinet. 1074 Platanthera hookeri, Hooker's Orchis(1). - - Cabinet. 1075 Platanthera flava, Small Pale-yellow Orchis(4). Cabinet. 1076 Platanthera hyperborea, Northern Orchis $(1)$. - - Cabinet. 1077 Pratanthera dilatata, Small White-flowered Orchis(4).Cabinet. 1078 Platanthera blephariglottis, Fringed White Orchis(3).Cabinet. 1079 Platanthera ciliaris, Fringed Yellow Orchis(2). - Cabinet. 1080 Platanthera psycodes, Purple Swamp Orchis(4). - Cabinet. 1081 Platanthera lacera, Ragged Orchis(2). - - - Cabinet. 
Latin Names.

Euglish Names.

1082 Platanthera braoteata, Green-flowered Orchis.

1083 Arethusa bugbosa, Arethusa(3). - - - Cabinet. 1084 Pogonia ophiogrossoides, Single leaved Pogonia(4). - Cabinet. 1085 Pogonia verticillata, Verticillate Pogonia(2). - - Cabinet. 1086 Pogonia pendula, Nodding Pogoria(3). - - Cabinet. 1087 Calopogon ptolchelitus, Calapogon(4). - - - Cabinet. 1088 Spiranthes Gracilis, Slender Lady's-tresses(7). - Cabinet. 1089 Spiranthes cernua, Nodding Lady's-tresses(4). - Cabihet. 1090 Spiranthes Plantaginea, Common Lady's-tresses(4). * Cabinet. 1091 Goonyera pubescens, Larger Rattlesnake Plantain(3). Cabinet. 1092 Goodyera repens, Smaller Rattlesnake Plantain(3). Cabinet. 1093 Listera cordata, Heart-leaved Twayblade(4). - Cabinet. 1094 Cypripedium pubescens, Large Yellow Ladystipper(1). Cabinet. 1095 Cypripedium Parviflorum, Smaller Yellow Ladyslipper(1).Cabinet. 1096 Cxpripedium spectabile, Showy Ladystipper(2). - - Cabinet. 1097 Cypripedidm aCaUle, Purple Ladyslipper, or Noah's-ark(2). Cab. 1098 Cyprifedium arietinum, Ramshead(1). - - - - Cabinet.

Common Stargrass(3). - Cabinet. ORDER CXIV. IRIDACEA.

1100 IrIS VERSICoLoR, Blue Flag(2). - - - - Cabinet. 1101 IRIS VIRGINICA, Slender Blue-flag(3). - a - Cabitet. 1102 Sisyrinchiom bermditana, Common Blueyed-grass(3). - Cabinet.

ORDER CXV. DIOSCOREACEA.

1103 Dioscorea viliosa, Wild Yamroot(4). - . - Cabinet.

ORDER CXVI. SMILACEA.

1104 Medeola virginica, Cucumber-root(3). - - - Cabinet. 1105 Tridilum erythrocarpum, Red-berried 3-leaved Nightshade(2).Cab. 1106 TrILLIUM CERNUUM, Nodding 3-leaved Nightshade(1).Cabinet. 1107 Trillidm erectum, False Wakerobin(5). - - - Cabinet. 1108 Trillium grandiforum, Large-flowered Trillium(2). - Cabinet. 
PLANTS.

Latin Names.

1109 Smilacina stellata,

1110 Smilacina trifolia,

1111 Similacina racemosa,

1112 Smilacina bifolia,
English Names.

Star-flowered Solomon's-seal(2). Cabinet.

Three-leaved Smilacina(4). - Cabinet.

Wild Spikenard(1). - - - Cabinet.

Two-leaved Solomon's-seal(7). Cabinet.

1113 Polygonatum multiflorum, Common Solomon's-seal(4). Cabinet.

1114 Clintonia boRealis,

1115 Clintonia umbegrata,

1116 Smilax Rotundifolia,

1117 Smilax Hispida,

1118 Smilax spinulosa,

1119 SMILAX HERBACEA,
Large-flowered Clintonia(3). Cabinet.

Small-flowered Clintonia.

Common Greenbrier(5). - - Cabinet.

Hispid Greenbrier.

Spinulose Greenbrier(2). - - Cabinet.

Carrionflower(1). - - - - Cabinet.

ORDER CXVII. LILIACEA.

1120 Lilium philadelphicum, Red Lily(4). - - - - - - Cabinet.

1121 Lilium canadense, Wild Yellow Lily(2). - - - Cabinet.

1122 LILIUM SUPER BUM, Superb Lily, or Turk's-cap(2). Cabinet.

1123 Erythroniom americanum, American Dogstoot/ Violet(3).Cabinet. 1124 Erythronium albidum, White Dogstooth Violet(2). - Cabinet. 1125 Ornithogalom umbeldatum, Common Star-of-Bethlehem(3). Cab.

1126 ALLIUM CANADENSE,

1127 AlLium Vineale,

1128 ALLIUM TRICOCCUM,

1129 ALLIUM CERNUUm,

1130 Asparagus ofFicinalis,

1131 ALETRIS Farinosa,
Meadow Garlic.

Wild Garlic(1). - - - - - Cabinet. Wild Leek(1). - - - - - Cabinet. Wild Onion(2). - - - - Cabinet. Common Asparagus(2). - - Cabinet. Colicroot(1). - - - - - Cabinet.

Vol. 39.]

ORDER CXVIII. PONTEDERIACE $A$.

1132 Pontederia cordata, Common Pickerelweed(2). - Cabinet. 1133 Heteranthera reniformis, Mud Plantain(2). - - - Cabinet. 1134 Heteranthera graminea, Water Stargrass(2). - - Cabinet.

ORDER OXIX. MELANTHACE疋.

1135 ZYGaDENUS GLAUCUS, Glaucous-leaved Zygadenus(2). Cabinet. 1136 Melanthidm virginicum, Virginian Melanthium(2). - Cabinet. 
Latin Names.

1137 Veratrum Viride,

1138 Melonias diotca,

1139 UvULARIA PERFolitata,

1140 Uvularia Grandiflora, Large-flowered Bellwort(1), - Cabinet. 1141 Uvularia sessiliffolia, Sessile-leaved Bellwort(2). - Cabinet. 1142 Prosartes lanugrnosa, Pale-flowered Prosartes(2). - Cabinet. 1143 Streptopus Amplexifolius, Smooth Twistedstalk(1). - Cabinet. 1144 Streptopus roseus,
English Names.

White Hellelore, or Indian Poke(2).Cabinet. Devilsbit, or Unicorn-plantt(2). Cabinet. Perfoliate Bellwort(4). - - Cabinet. Rose Twistedstalk(1). - - - Cabinet.
1145 LUZULA CaMPESTRIS,

1146 LuzUla PIlosa,

1147 Luzula parviflora,

1148 JUNCUS EFFUSUS,

1149 JunCUS FILIFORNIIS,

1150 Juncus balticus,

1151 Juncus Nodosus,

1152 JUNCUS POLYCEFHALUS,

1153 Juncus acuminatus,

1154 Juncus pelocarpus,

1155 Juncus CONRadi, 1156 Juncus Bufonius,

1157 Juncus tenuis,

1158 JUNCUS GREENEI,

1159 JunCUS GERARDI,

1160 Juncus marginatus,

1161 Juncus STYGIUs, 1162 Juncus trifidus,
Common Woodrush(4). - - Cabinet. Broad-leaved Hairy Woodrush(4).Cabinet. Small-flowered Woodrush(2). Cabinet. Soft Rush(2). - - - - Cabinet. Slender Rush(4). - . - Cabinet. Baltic Rush(4). - - - - Cabinet. Smaller Roundheaded Rush(9). Cabinet. Many-headed Rush(6). - - Cabinet. Sharp-fruited Rush(3). - Cabinet. Brownish-fruited Rush.

Conrad's Rush(6). - - . Cabinet. Toad Rush(2). - - - - Cabinet. Slender Rush(6). - - - Cabinet. Greene's Rush. Blackgrass(3). - - - C Cabinet. Grass-leaved Rush(4). - - Cabinet. Large-fruited Rush(6). - - Cabinet. Slender-fringed Rush(6). - Cabinet.

ORDER OXXI. COMMELYNACEZE.

1163 Commelyna angustrolia, Narrow-leaved Dayflower.

1164 Tradescantra virginica, Virginian Spiderwort(1). - Cabinet.

ORDER OXXII. XYRIDACEAE.

1165 Xyris caroliniana,

Common Yelloweyed-grass(3), Cabinot. 


\section{ORDER CXXIII, ERIOCAULONACEA.}

Latin Names.

Englisin Names.

1166 Eriocaulon septangulare, Pellucid Pipewort(4). - - Cabinet.

Vol. 40.]

ORDER CXXIV, CYPERACEA.

1167 Dolichidm spathaceum, Dulichium(3). - - - Cabinet.

1168 Cyperus flavescens,

Yellowish Dwoarf Galingale(4). Cabinet.

1169 Cyperus diandrus,

1170 Cyperus nutTalțil, Diandrous Galingale(15). - Cabinet.

1171 Cyperus michauxianus, Nuttall's Galingale(3). - - Cabinet.

1172 Cyperus strigosus,

1173 Cyperus refens,

1174 Oyperus fiłiculmis,

1175 Cyperus gr.AYi,

1176 Cyperus dentatus,

2177 Cyperus inflexus,

1178 Cyperus schweinitziI,

1179 Cyperts OVUTtaris,

1180 Cyperds RETrofractUs,

1181 FUURENA SQUARHOSA,

1182 Euneocharis Palustrịs,

Michaux's Galingale(1). - - Cabinet. Tall Galingale(6). - - - Cabinet. Creeping Galingale(1). - - Cabinet. Slender-stalked Galingale(4). Cabinet. Gray's Galingale(2). - - " Cabinet. Toothed Galingale(3). - - Cabinet. Dwarf Odorous Galingale(7), Cabinet. Schweinitz's Galingale(2). - Cabịnet. Oval-headed Galingale(2), - Cabịnet. Bent-flowered Galingale.

Squarrose Fuirena(7). - - Cabinet. Common Spikerush(7). - - Cabinet. 1183 Eụeocharis olivacea, Olive-fruited Spikerush(2). - Cabinet. 1184 Eleocharis rosteltata, Beaked Spikerush(2). - - Cabinet. 1185 Eteocharis intermedia, Intermediate Spikerush(3). - Cabinet. 1186 Eleocharis obtusa, Oltuse-headed Spikerush(4). Cabinet. 1187 Elfocharis tuberculosa, Large-tubercled Spikerush(2). Cabinet. 1188 Eleocharis aCiCULARIs, Capillary Spikerush(6). - - Cabinet. 1189 ELEOCHaRIS TENUIS, Slender Spikerush(1). - - Cabinet.

1190 Eleocharis pygm $\approx$ e, 1191 Scirpus planifolius, Dwarf Spikerush(8). - - Cabinet. 1192 Scirpos subterminalis, Floating Clubrush(2). - - Cabinet. 1193 Scirpus CESPItosus, 1194 Scirpus Debilis, Scaly-stalked Clubrush(3). - Cabinet, Weak-stalked Clubrusk(4). - Cabinet. 1195 Scirpus triqueter,

Chairmaker's Rush(7). - - Cabinet. 
Latin Names.

1196 Scirpus mucronatus,

1197 ScikPUS LaCUStris,

1198 Scirpus maritrmus,

1199 Scirpus atrovirens,

1200 ScIRPUS BRUNNEUS,

1201 Scirpus ERIOPHORUS,

1202 Scirfus Lineatus,

1203 ERTophorUm aLPINUM,
English Names.

Longheaded Triangular Rush(1).Cabinet. Bulrush(3). - - - - - Cabinet. Sea Clubrush(3). - - - Cabinet. Dark-green Clubrush(2). - - Cabinet. Brown-headed Clubrush(1). - Cabinet. Brown Woolgrass(3). - - - Cabinet. Loose-flowered Woolgrass(2). Cabinet. Alpine Cottongrass(3). - - Cabinet. 1204 Eriophorum vaginatum, Harestail(3). - - - - - Cabinet. 1205 Erlophorum virginicum, Rusty Cottongrass(3). - - Cabinet. 1206 Eriophorum polystachyom, Broad-leaved Cottongrass(3),Cabinet. 1207 Eriophordm angustifolium, Narrow-leaved Cottongrass(5). Cab. 1208 Fimbristylis spadicea, Tall Brovn-spiked Fimbristylis(2). Cab. 1209 Isolepis capillaris, Hair-like Isolepis(2). - - - Cabinet. 1210 Trichelostylis mucrondlata, Common Trichelostylis(6). Cabinet. 1211 Hemicarpha subsquarrosa, Dwarf Hemicarpha(6). - Cabinet. 1212 Rhynchospora alba, White Beakrush(2). - - - Cabinet. 1213 Rhynchospora capillacea, Small Capillary Beakrush(3).Cabinet. 1214 Rhynchospora fusca, Brown Beakrush(5). - - - Cabinet. 1215 Rhynchospora gracilenta, Taill Slender Beakrush(1). Cabinet. 1216 Rhynchospora glomerata, Common Beakrush(3). - - Cabinet. 1217 Rhynchospora cephalantha, Roundheaded Beakrush(2). Cabinet. 1218 Cladium Mariscoides, Smooth Twigrush(4). - - - Cabinet. 1219 Sclegia retictlaris, 1220 Scleria laxa, 1221 Scleria triglomerata, 1222 Scleria pauciflora, 1223 Scleria Verticlliata, Sessile-spiked Nutrush(2)- - Cabinet. Loose-flowered Nutrush(4). - Cabinet. Three-clustered Nutrush(4). - Cabinet. Feu-flowered Nutrush(2). - Cabinet. Dwarf Verticillate Nutrush(2). Cabinet.

Vol. 41.]

1224 Carex DIOTCa, 1225 CaReX exizIS, 1226 CareX Paudiflora,
Diacious Sedge(1). - - - Cabinet. Slender Sedge(5). - - - Cabinet. Few-flowered Sedge(6). - - Cabinet. 
Latin Names.

1227 CaREX POLYTRICHOIDES,

1228 Carex Willdenovit,

1229 Carex backit,

1230 Carex disperma,

1231 CAREX CHORDORRHIZA,

1232 CaREX CEPhaLophora,

1233 Carex muhlenbergit,

1234 Carex siccata,

1235 CaREX ROSEA,

1236 Carex Retroflexa,

1237 Carex sparganioIdes,

1238 Carex stipata,

1239 Carex vulpinotdea,

1240 Carex setacea,

1241 Carex bromotdes,

1242 Carex alopecoidea,

1243 CAREX SARTWELLII,

1244 Carex teretiuscula,

1245 CaRex decomposita,

1246 Carex trisperma,

1247 Carex DEWEyaNa,

1248 Carex canescens,

1249 Carex stellulata,

1250 Carex tenuifloka,

1251 Carex scoparia,

1252 Carex straminea,

1253 Carex pedunculata,

1254 CareX squarrosa,

1255 Carex buXbaumir,

1256 CAREX TRICEPS,

1257 Carex VIREscens,

1258 CAREX GRACILLIMA,
English Names.

Bristle-stalked Sedge(3). - - Cabinet. Willdenow's Sedge(5). - - Oabinet.

Back's Sedge(2). - - - Cabinet.

Two-seeded Sedge(2). - - - Cabinet.

Long-rooted Sedge(6). - - Cabinet.

Oval-headed Sedge(5). - - Cabinet.

Muhlenberg's Sedge(3). - - Cabinet.

Dry-spiked Sedge(3). - - - Cabinet.

Rose $S_{\epsilon}$ dge(4). - - - - Cabinet.

Retroflexed Sedge(2). - - - Cabinet.

Bur-reed Sedge(6). - - - Cabinet.

Awl-fruited Sedge(3). - - Cabinet.

Fox Sedge(8). - - - - Cabinet.

Bristly-spiked Sedge.

Bromus-like Sedge(9). - - Cabinet.

Foxtail Sedge.

Sartwell's Sedge(3). - - - Cabinet.

Lesser-panicled Sedge(5). - Cabinet.

Large-paricled Sedge(3). - Cabinet.

Three-seeded Sedge(2). - - Cabinet.

Dewey's Sedge(3). - - - Cabinet.

White Sedge(6). - - - Cabinet.

Little Prickly Sedge(2). - - Cabinet.

Slender Cluster-spiked Sedge(2).Cabinet. Broom-like Sedge(10). - - Cabinet. Straw-colored Sedge(19). - Cabinet. Long-stalked Sedge(4). - - Cabinet. Squarrose-headed Sedge(4). - Cabinet. Buxbaum's Sedge(4). - - - Cabinet. Three-headed Pubescent Sedge(5).Cabinet. Green-spiked Pubescent Sedge(4).Cabinet. Stender Nodding Sedge(3). - Cabinet. 
I atin Names.

1259 Carix formosa,

1260 Carex davisir,

1261 Carex rigida,

1262 Carex angustata,

1263 Carex cespitosa,

1261 Carex aquatilis,

1265 Carex atJREA,

Vol. 42.]

1266 Carex crinita,

1267 OAREX OLIGOSPERMA

1268 Carex inflata,

1269 Carex cyLINDrica;

1270 Carex utriculata,

1271. Carex subulata,

1272 Carex folliculata,

1273 Carex. intumescens,

1274 Carex lupulina,

1275 Carex scabrata,

1276 Carex schWeinitzí,

1277 Carex RETRorsa,

1278 Carex tentaculata,

1279 CAREX HYSTRICINA,

1280 Carex pseudo-cyperus,

1281 Carex longirostris,

1282 CAREX TRICHOCARPA,

1283 Carex aristata,

1284 Carex unbeliata,

1285 Carex pennsylvanica,

1286 Carex nover-ANGLIE,

1287 Carex filiformis,

1288 Carex lanuginosa,

1289 Carex vestita,

1290 Carex pUBdesoens,
English Names.

Showy Sedge(3). - - - Cabinet.

Davis's Sedge(3). - - - - Cabinet.

Rigid Sedge(4). - - - - Cabinet.

Large Bog Sedge(3). - - - Cabinet.

Smaller Bog Sedge.

Water Sedge(2). - - - Cabinet.

Golden-fruited Sedge(6). - Cabinet.

Fringed Sedge(4). - - - - Cabinet.

Few-fruited Sedge(3). - - Cabinet.

Inflated Sedge.

Cylindrical-spiked Sedge.

Bladder-fruited Sedge(9). - Cabinet. Awl-fruited Sedgc(4). - - Cabinet. Tall Yellow Sedge(7). - - Cabinet.

Swollen-fruited Sedge(4). - Cabinet. Hop Sedge(4). - - - - Cabinet. Rough-fruited Sedge(3). - - Cabinet. Schweinitz's Sedge(2). - - Cabinet Backward-fruited Sedge(2). - Cabinet. Long-pointed Sedge(3). - - Cabinet. Porcupine Sedge(5). - - - Cabinet. Cypress-like Sedge.

Long-beaked Sedge(3). - - Cabinet. Hairy-fruited Sedge.

Awned Sedge(3). - - - - Cabinet.

Umbel-spiked Sedge(8). - - Cabinet.

Pennsylvanian Sedge(7). - - Cabinet.

New-England Sedge(3). - - Cabinet.

Slender-leaved Sedge(3). - - Cabinet.

Woally-fruited Sedge(9). - Cabinet.

Short Woolly-spiled Sedge(4). Cabinet.

Pubescent Sedge(3). - . - Oabinet. 
PLANTS.

Latin Names.

1291 Carex limosa,

1292 Carex livida,

1293 CaREX FLAVA,

1294 CanEX EDERI,

1295 Carex pallescens,

1296 CAREX TORREYI,

1297 Carex striata,

1298 Carex granUlaris,

1299 CaREX LAXIFLoRA,

1300 Carex conoidea,

1301 Carex digitalis,

1302 Carex oligocarpa

1303 Carex tetanica,

1304 Carex anceps,

1305 Carex rLanda,

1306 CaREX CRAWEI,

1307 Carex plantaginea,

1308 Carex careyana,

1309 Carex eburnea,

1310 Carex FLEXILIS,

1311 Carex arctata,

1312 CaREX DEBILIS,

1313 Carex miliacea,

1314 Carex lacustris,
English Names.

Mud Sedge(12). - - - - Cabinet.

Livid Sedge(8). - - - Cabinet.

Large Yellow Sedge(6). - - Cabinet.

QEder's Sedge(2). - - - - Cabinet.

Pale Pubescent Sedge(5). - Cabinet.

Torrey's Sedge.

Striated Sedge(2). - - - - Cabinet.

Granular-spiked Sedge)4). - Cabinet.

Loose-flowered Sedge.

Conical-fruited Sorge(2). - Cabinet.

Slender Sedge(7). - - - - Cabinet.

Small Few-fruited Sedge(9). Cabinet.

Crooked-necked Sedge(3). - Cabinet.

Two-edged Sedge(14). - - Cabinet.

Pale Smooth Sedge.

Crawe's Sedge(2). - - - Cabinet.

Plantain-leaved Sedge(4). - Cabinet.

Carey's Sedge(2). - - - Cabinet.

Bristle-leaved White Sedge(5). Cabinet.

Fringed Sedge(4). - - - - Cabinet.

Short-beaked Wood Sedge(3). Cabinet.

Weak Sedge(3). - - - - Cabinet.

Millet-like Sedge(5). - - - Cabinet.

Lake Sedge(3). - - - - Cabinet.
Vol. 43.]

1315 LeErsia oryzoides,

1316 LEERSIA VIRGINICA,

1317 Zizania aquatica,

1318 Alopectrus pratensis,
ORDER CXXV. GRAMINE $\boldsymbol{A}$.

Cutgrass, or Whitegrass(2). Cabinet. Small-flowered Whitegrass(2). Cabinet. Wild Rice(2). - - - - Cabinet.

Common Foxtail-grass.

1319 Alopecurus geniculatus, Water Foxtail-grass(3). - - Cabihet.

.1820 Phleóm pratense,

Timothy, or Herdsgrass(2). - Cabinet.

1321 Phalaris arundinacea, Reed Canary-grass(2). * Cabinet. 
Latin Names.

1322 Phalaris canariensis,

1323 Holcus lanatus,

1324 Hierochloa borealis,

1325 Hierochloa alpina,
English Names

Common Canary-grass(1). - Cabinet. Meadow Soflgrass(3). - - Cabinet. Seneca or Vanilla Grass(2). - Cabinet. Alpine Holygrass(4). - - - Cabinet.

1326 Anthoxanthum odoratum, Sweetscented Vernal-grass(5). Cabinet.

1327 Paspalum late,

1328 Paspalum setaceum,

1329 Milium effusum,

1330 Panicum sanguinale,

1331 Panicum glabrum,

1332 Panicum filiforme,

1333 Panicum crus-gali,

1334 Panicdm virgatum,

1335 Panicum latifolium,

1336 Panicum clandestinum, Hidden-flowered Panicgrass(6). Cabinet.

1337 Panicum xanthophysum, Yellow Panicgrass(2). - - Cabinet.

1338 Panicum CaPILLaRe,

1339 Panicum depauperatum, Few-flowered Panicgrass(4). Cabinet.

1340 Panicum pichotomum,

1341 Panicum verrucosum,

1342 Panicum agrostoldes,

1343 Panicum proliferum,

1344 Pennisetum glaucum,

1345 Pennisetum viride,

1346 Cenchrus tribuloides,
Hair-stalked Panicgrass(5). Cabinet.

Smooth Erect Paspalum(2). - Cabinet.

Hairy Slender Paspalum(2). Cabinet. Millet-grass(2). - - - - Cabinet.

Common Crabgrass(1). - - Cabinet.

Smooth Crabgrass(2). - - Cabinet.

Slender Crabgrass(2). - - Cabinet.

Barnyard-grass(4). - - Cabinet.

Tall Smooth Panicgrass(2). Cabinet.

Broad-leaved Panicgrass(3). Cabinet.

Polymorphous Panicgrass(22). Cabinet.

Warty-flowered Panicgrass(3). Cabinet. Agrostis-like Panicgrass(3). Cabinet. Prolifferous Panicgrass(2). - Cabinet. Foxtail, or Bottlegrass(2). - Cabinet. Green Bottlegrass(1). - - - Cabinet. Burgrass(4). - - - - Cabinet.

Vol. 44.]

1347 Oryzopsis asperifolia, White-grained Mountain-rice(4).).Cabinet. 1348 Oryzopsis melanocarpa, Black-fruited Mountain-rice(2).Cabinet. 1349 Oryzopsis canadensis, Smallest Mountain-rice(2). - Cabinet. 1350 Stipa avenacea, Black Oatgrass(1). - - - Cabinet. 1351 Aristida dichotoma, Dichotomous Threeawned-grass(2).Cabinet. 1352 ARISTIDA GRACILIS, Slender Threeawned-grass(3). Cabinet. 
Latin Names.

\section{English Names.}

1353 Munlenbergia diffusa, Dropseed-grass(3). - - - Cabinet. 1354 Munlenbergia cinna, Tall Muhlerbergia.

1355 Munlenbergia willdenovis, Willdenow's Muhlenbergia(3).Cabinet. 1356 Muhlenbergia sylvatica, Wood Muhlenbergia(3). - - Cabinet. 1357 Muhlenbergia sobolifera, Awnless Muhlenbergia.

1358 Muhlenbergia glomerata, Cluster-spiked Muhlenbergia(3). Cab.

1359 Muhlenbergia mexicana, Mexican Muhlenbergia(2). - Cabinet.

1360 VILFA VAGINEFLORA,

1361 VILFA ASPERA,

1362 Vilfa serotina,

1363 VILFA Heterolepis,

1364 VILFA CRYPTANDRA,

1365 Agrostis VULGaris,

1366 Agrostis alba,

1367 Agrostis LAXIFLORA,

1368 Agrostis stricta,

1369 Agrostis canina,
Hidden-flowered Vilfa(4). - Cabinet. Rough-leaved Vilfa(2). - Cabinet. Late-floutering Vilfa(3). - - Cabinet. Strong-scented Vilfa.

Large-panicled Vilfa(1). - - Cabinet. Herdsgrass.

Fiorin-grass.

Hairgrass (6). - - - - Cabinet. Upright-flowered Bentgrass(2). Cabinet. Bentgrass(3). - - - - Cabinet. 1370 Brachyelytrum aristatum, Auned Brachyelytrum(2). Cabinet. 1371 Calamagrostis canadensis, Canadian Smallreed(5). - Cabinet. 1372 Calamagrostis coarctata, Glaucous Smallreed(2). - Cabinet. 1373 Calamagrostis inexpansa, Close-flowered Smallreed(2). Cabinet. 1374 Ammophita arundinacea, Common Seareed(2). - - - Cabinet. 1375 Phragmites communis, Common Reed(1). - - - Cabinet. 1376 Eleusine INdica, Dogstail-grass, or Yard-grass(3).Cabinet. 1377 Spartina cynosuroides, Tall Marshgrass(2). - - - Cabinet. 1378 Spartina juncea, Rush-like Marshgrass(3). - Cabinet. 1379 Spartina alterntfolia, Smooth Marshgrass(2). - - Cabinet. 1380 Bouteloua racemosa, Racemed Bouteloua(2). - - Cabinet. 1381 Arra fleXUosa, 1382 Aira atropurpurea, 1383 Aira cespitosa, 1384 Trisetum molle,
Common Hairgrass(3). - Cabinet.

Purple Alpine Hairgrass(3). Cabinet. Tufted Hairgrass(5). - - - Cabinet. Soft Trisetum(3). - - - Cabinet. 
Latin Names.

English Names.

1385 Avena Pennsylvanicum, Pennsylvanian Wildoat(3). - Cabinet. 1386 Avena striata, Purple Wildoat(2), - - Cabinet. 1387 Arrhenatherum avenaceum, Grass-of-the-Andes(2). - Cabinet 1388 Danthoma spicata, Wild Oatgrass(3). - - Cabinet. 1389 Uralepis aristulata, Short=awned Uralepis(3). - Cabinet. Vol. 45.]

1390 Poa annta, Aınual Meadowgrass(5). - Cabinet. 1391 POA LAXA, 1392 Poa trivialis, 1393 Poa pratensis, 1394 Poa compressa, 1395 PoA PUngens, 1396 Poa nemoralis, 1397 Poa serotina, 1398 PoA Debilis, 1399 Poa eragrostis, 1400 POA P1LOSA, 1401 Poa reptans, 1402 Poa hirsuta, 1403 Poa capilitaris, 1404 Poa dentata, 1405 Poa michadXII, 1406 Tricuspis SESLerioldes, 1407 Glyceria Fluitans, 1408 Glyceria actifitiora, 1409 GLiyCERIA AQUATICA, 1410 Glyceria nervata, 1411 Glyceria elongata, 1412 Grycerla canadensis,

1413 Dactylis glomerata, 1414 Bromus secalinus, 1415 Bromus ciliatus, 1416 Bromus purgans, Few-flowered Alpine Meadowgrass(3).Cabinet. Rough Meadowgrass(1). - - Cabinet. Smooth-stalked Meadowgrass(3).Cabinet. Bluegrass, or Wiregrass(7). Cabinet. Sharp-leaved Meadowgrass(2). Cabinet. Wood Meadowgrass(1). - - Cabinet. Redtop(2). - - - - - Cabinet. Weak Meadowgrass(3). - - Cabinet. Ștrongscented Meadowgrass(1). Cabinet. Slender Meadowgrass(2). - Cabinet. Creeping Meadowgrass(4). - Cabinet. Hairy Meadowgrass(1). - - Cabinet. Hair-panicled Meadowgrass(2). Cabinet. Toothed Meadowgrass(2). - Cabinet. Spiked Saltmarsh Poa(3). - Cabinet. Tall Redtop(2). - - Cahinet. Common Mannagrass(1). = Cabinet. Sharp-flowered Mannagrass(2), Cabinet. Reed Mannagrass(1). - - - Cabinet. Nerved Mannagrass(6). - - Cabinet. Long-panicled Mannagrass(2). Cabinet. Rattlesnake-grass(2): - - - Cabinet. Cocksfoot-grass(2). - - - Cabinet. Cheat, or Chess(2). = - - Cabinet. Fringed Bromegrass(4). - - Cabinet. Hairy-flowered Bromegrass(2). Cabinet. 
PLANTS.

Latin Names.

English Names.

1417 Bromus steriLIs, Barren Bromegrass(1). - " Cạbinet.

1418 Kgleria pennsylvanica, Pennsylvanian Kaleria(2). - Cabinet.

1419 Kaleria truncata,

1420 Festuca dURIUscula,

1421 Festuca tenella,

1422 Festuca nutans,

1423 FEstuCa ELATIOR,

1424 Festuca pratensis,

1425 Diplachne fascicularis,

1426 UNIOLA GRACILIS,

1427 LoLIUM PERENNe,

1428 Triticum REPENS,

1429 Triticum caninum,

1430 Elymus virginicus,

1431 Elymus canadensis,

1432 Elymus villosus,

1433 Elymus hystrix,

1434 ANDROPOGON NUTANS,
Truncated Kcoleria(2). - - Cabinet, Hard Fescuegrass(3). - - - Cabinet.

Slender Fescuegrass(2). - - Cabinet. Nodding Fescuegrass(2). - - Cabinet. Tall Fescuegrass(1). - - - Cabinet. Meadow Fescuegrass(2). - - Cabinet. Cluster-flowered Diplachne(2). Cabinet. Slender Spikegrass(2). - - Cabinet. Common Darnel(1). - - - Cabinet. Couchgrass, or Quitchgrass(2). Cabinet. Fibrous-rooted Wheatgrass(2). Cabinet. Virginian Lymegrass(2). - Cabinet. Canadian Lymegrass(2). - - Cabinet. Slender-hairy Lymegrass(2). Cabinet. Bottlebrush-grass(4). - - - Cabinet. Indian Beardgrass.

1435 Andropogon scoparius, Purple Woodgrass(2). - - Cabinet. 1436 Andropogon furcatus, Finger-spiked Woodgrass(2). Cabinet. 1437 ANdropogon virginicus, Virginian Beardgrass(3). - Cabinet. 1438 Andropogon macrourus, Cluster-flowered Beardgrass(2). Cabinet. 


\section{A D D I T I 0 N S.}

PLANTS DISCOVERED DURING THE PRINTING OF THE FLORA, And deseribed in Vol. ii, pp. 515 ot seq.

Latin Names.

1439 Polygala tutea,

1440 Desmodium lexvigaturm, Rigid Upright Desmodium.

144.1 Lythrum sadicaria,

1442 Saxifraga aizoides,
Common Purple Loosestriffe.

Yellow Mountain Saxifrage.

1443 Eupatoridm hyssopifolium, Hyssop-leaved Hempweed.

1444 Eufatoriun album, Whiteheaded Hempweed.

1445 Eupatorium resinosum, Resinous Hempweed.

1446 Aster concolor,

1447 OAKesIa conradi,

1448 Calypso borealis,

1449 Eleocharis melanocarpa, Black-fruited Eltocharis(2). Cabinet.

Racemed Violet Aster.

Conrad's Oakesia(3). - - Cabinet.

Northern Calypso.
PLANTS DISCOVERED AND COLLECTED SINCE THE PUBLICATION OF THE FLORA.

1450 RoBINIA vISCOSA, Clammy Locust (1). - - Cabinet.

1451 Victa caroliniana, Carolinian Vetch(1). - - Cabinet. 1452 Stellaria longipes, Long-stalked Stitchwort(1): - Cabinet. 1453 Trifolium incarnatum, Incarnate Clover(2). - - Cabinet. 1454 Pyrus malus, Common Apple(4). - - - = Cabinet. 1455 CENothera RIPARIA, 1456 Galium concinnum, Concinnute Bluets $(1)$ : = - Cahinet. 
Latin Names.

1457 Phlomis tuberosa,
English Names.

Tuberous Phlomis(2). - - - Cabinet.

1458 Lithospermum latifolium, Broad-leaved Gromwell(1). Cabinet.

1459 Onosmodium carolinianum, Carolina False-gromuell(2). Cabinet.

1460 Amaranthus tamariscinus, Salt Amararth(1). - - Cabinet.

1461 Chenopodium murale, Wall Goosefoot(3). - - - - Cabinet.

1462 Salix fragiLIs,

1463 Salix babylonica,

1464 Salix eriocephala,

1465 Salix angustata,

1466 Melanthium hybridum,

1467 CAREX STEUdeLiI,

1468 Carex panicdlata,

1469 Carex volgaris,

1470 Carex torta,

1471 Carex grisea,

1472 Carex platyphylla,

1473 Carex comosa,

1474 Panicum faucifloruni,

1475 Avena sativa,

1476 Spartina polystachya,

1477 Cinna pendula,

1478 Cinna arundinacea,
Brittle Willour(8). - - Cabinet.

Weeping Willow(2). - - Cabinet.

Woolly-head Willow(3). - - Cabinet.

Narrous-leaved Willow(3). - Cabinet.

Hybrid Melanthium(1). - - Cabinet.

Steudel's Sedge(1). - - Cabinet.

Panicled Sedge(1). - - - Cabinet.

Small Black-scaled Sedge(1). Cabinet.

Twisted Sedge(6). - - - Cabinet.

Greyish Sedge(4). - - - Cabinet.

Flat-leaved Sedge(2). - - - Cabinet.

Cyprus-like Sedge(3). - - Cabinet.

Few-flowered Panicgrass(2). Cabinet.

Common Oat(2). - - - Cabinet.

Tall Saltmarsh-grass(2). - Cabinet.

Pendulous Cinna(2). - - - Cabinet.

Reed-like Cinna(2). - - - Cabinet. 



\title{
BOTANY OF NEW-YORK,
}

OR THE

\section{$\mathbb{N} \mathfrak{e} \mathfrak{w} \sim \mathfrak{1} \mathfrak{a} \mathfrak{x} \mathfrak{i} \quad \mathfrak{F} \mathfrak{l} \mathfrak{a} \mathfrak{x} \mathfrak{a}$}

\author{
BY JOHN TORREY.
}

DIVISION II, CRYPTOGAMOUS PLANTS. 



\section{B 0 T A N.}

\section{DIVISION II. CRYPTOGAMOUS PLANTS,}

Vol. 46.] ORDER CXXVI. EQUISRTACEA.

Latin Name.

English Name.

In the

1 Equisetum arvense, Field Horsetail.

2 Equisetum sylvaticum, Wood Horsetail(7). - - Cabinet.

3 Equisetum limosun, Smooth Swamp Horsetait, or Pipes(3).Cabinet.

4 Equisetum hyemale, Scouring Rush(4). - - - Cabinet.

5 Equisetum variegatum, Variegated Rough Horsetail(5).Cabinet

6 Equisetum scirpoides, Smallest Rough Horselail(3). Cabinet.

ORDER CXXYII. FILICES.

7 Polypodium vulgare, Common Polypody(2). - - Cabinet.

8 Polypodidm phegopteris, Beecl Polypody(2). - - Cabinet;

9 Polypodium hexagonopterum, Winged Polypody(1). - - Cabinet.

10 Polypodidm dryopteris, Three-branched Polypody(2). Cabinet.

11 Struthiopteris germanica, Common Ostrichfern(4). - - Cabinet.

12 Allosorus gracilis, Slender Allosorus(4). - - Cabinet:

13 Adintum pedatum, Maidenhair, or Mohair(1). - Cabinet.

14 Pteris aquilina, Common Brake, or Bracken(2). Cabinet.

15 Platyloma atropurpurea, Purple-stalked Rockbrake(2). Cabinet.

16 Doodia virginica, Virginian Doodia(1). = - Cabinet.

17 Woodwardia angustifolia, Narrow-leaved Woodwardia(2). Cabinet.

18 Scolopendrum officinardm, Common Houndstongue(2). - Cabinet.

19 Asplenidm trichomanes, Small Rock Spleenwort(3). - Cabinet. 
Latin Names.

20 Asplentum ebeneum,

21 Asplenium angustifolium, Narrow-leaved Spleenwort(2). Cabinet.

22 Asplenitm ruta-muraria, Wall-rue Spleenwort(3). - - Cabinet.

23 Asplenium thelypterioides, Silvery Spleenuort(1). - - Cabinet.

24 Asplenium Filix-Fcimina, Female-fern(1). - - - Cabinet.

25 Antigramia rhlzophxlda, Walking-fern(5). - - - Cabinet,

26 Aspidium Marginale,

27 Aspidium goldiantm,

28 Aspidium CRIstatum,

29 Aspidium dilatatum,

30 AsPidiUM THELYPTERIs,

31 Aspidium NOVEBoracense,

32 Aspidium acrostichordes,

33 Aspidium aculeatum,

34 Onoclea sensibilis,

35 Woodsia ILVENSIS,

36 WOODSIA OBTUSA,

37 Cystoyteris Fragills,

38 Cystopteris buleifera,

39 Dicrsonia pilosiuscula,

40 OsMunda cLaytoniana,

41 Osmunda cinnamomea,

42 Osmunda spectabilis,

43 Lygodium palmatum,

44 Ophioglossum vulgatum,

45 BotrychiUm viRgINICUM,

46 BotrychIUM LUNARIOIDES,

47 Botr ychiuM SIMPLEX,
Marginal Shieldfern(1). - - Cabinet.

Goldie's Shieldfern(2). - - Cabinet,

Crested Shieldfern(1). - - Cabinet.

Dilated Shieldfern(1). - - Cabinet.

Meadow Shieldfern(1). - - Cabinet,

New-York Shieldfern(1). - Cabinet.

Terminal Shieldfern(2). - - Cahinet.

Prickly Shieldfern.

Sensitive-fern(4): - - - Cabinet.

Rusty Rock Polypody;

Obtuse Woodsia(3), - - " Cabinet.

Brittle Bladderferu(4). - - Cabinet.

Bulb-bearing Bladderfern(2). Cabinet.

Hairy Dicksonia(2). - - Cabinet.

Interrupted Flowering-fern(1). Cabinet. Woolly Flowering-fern(2). - Cabinet.

Showy Flowering fern(2). - Cabinet. Climbing-ferro(1). - - - Cabinet.

Common Adderstongue(2). - Cabinet.

Rattlesnake-fern(1). - - Cabinet.

Tall Smooth Moonwort(2). - Cabinet.

Dwarf Moonwort(1). - . - Cabinet.

ORDER CXXVIII. LYCOPODIACEXT.

48 LxCOPODIUM SELAGo,

49 Lycopoditm Lucidulum,

50 Lycopopium inUNDaTUM,
Fir Clubmoss(3). - - - Cabinet.

Shining Clubmoss(3). - - - Cabinet.

Low Marsh Clubmoss(7). - - Cabịnet; 
PLANTS.

Latin Names.

51 Lycopodium annotinum,

52 Lycopodium obscurum,

53 Lxcopodium clavatum,

English Names.

54 Lxcopodium complanatum, Festoon Groundpine(2). - - Cabinet.

55 Selaginelda Rupestris, Small Rock Clubmoss(4). - Cabinet.

56 Selaginella apus,

Moss-like Selaginella(4). - - Cabinet.

\section{ORDER CXXIX, SALVINIACE正.}

57 Salvinia natans, Floating Salvinia.

58 Azolta caroliniana,

Carolinian Azolla(3). - - Cabinet.

ORDER CXXX. ISOETACE E.

59 Isomtes LAACUSTRt?

Common Quillwort(1). \% = Cabinet. 



\title{
MINERALOGY OF NEW-YORK,
}

\author{
BY LEWIS C. BECK.
}





\section{MINERALOGY.}

\section{CLASS I. GASEOUS MINERALS.}

\section{ORDER 1. COMBUSTIBLE GASES.}

Names of Species.

Localities.

1 Hydrogen (Not found pure).

2 Carburetted hydrogen (See Mineralogy, p. 128).

3 Sulphuretted hydrogen (See Mineralogy, p. 141).

4 Phosphuretted hydrogen (Sometimes found in markhes).

ORDER II. NON-COMBUSTIBLE GASES.

5 OXYGEN (Sometimes found to predominate in the air given out by springs).

6 Nitrogen (See Mineralogy, p. 133).

7 Carbonic actd (See Mineralogy, p. 134)،

8 ATMospheric aIR (Sometimes evolved from springs).

CLASS II. LIQUID MINERALS, NOT COMBUSTIBLE.

ORDER I. LIQUID ACIDS.

9 Hydrous sulphuric acid (See Mineralogy, p. 150).

ORDER II. LIQUID MINERALS, NOT ACID.

10 Atmosphekic water (See Mineralogy, p. 178).

11 Sea water (See Mineralogy, p. 179). 


\section{CLASS III. COMBUSTIBLE MINERALS, NOT GASEOUS.}

Names.

12 SULFHUR (1 specimen),

13 Bitcmen : Petroleum(1),

14 AMber,

15 Graphite (19 specimens),

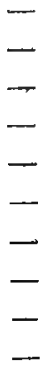

- Artificial

Localities.

In the

Springport, Essex county. - - Cabinet.

Rossville, Richmond county.

Rogersrock, Essex, 2 specimens;

Ticonderoga, Essex, 3;

Port Henry, Essex, 2;

Fishkill, Dutchess, 4;

Brown's quarry, Putnam, 1:

Edenville, Orange, 2;

Saltus's quarry, Essex, 1;

Rossie, St. Lawrence, 1;

Johnsburgh, Warren, 2;

16 Anthiacite $(5)$;

Rossie furnace, St.Lawrence, 1.

Littlefalls, Herkimer, 2;

Sprakers, Montgomery, :-;

Middleburgh, Schoharie, 1 .

17 Coal (See Mineralogy; p. 190).

18 Lignit'e (See Mineralogy; p. 191).

19 Peat(1),

Lansingburgh, Rensselaer. - Cabinet.

\section{CLASS IV. ALKALINE MINERALS.}

ORDER I. AMMONIA.

20 Carbonate of ammonia, In New-York city.

ORDER II: SODA.

21 GLauber's gatT;

In Rochester, Monroe.

22 Natron,

New-York city ${ }_{i}$ etc.

23 Common salt (See Mineralogy, p. 99).

24 Hrdriodate of SODA, Probably in the brine springs of Onondaga.

ORDER III. POTASH.

25 Sulphate of Potash, Perhaps in the brine of Salina.

26 Carbonate of POtash, Water of certain springs. 
Names.

Localities.

27 Muriate of potash, Water of brine springs, etc.

28 Hydrobromate of potash, Waters of Saratoga.

29 Alum,

Catskill mountains, etc.

\section{CLASS V. ALKALINE EARTHY MINERALS,}

ORDER I, BARYTA.

30 HFavy spar(35),

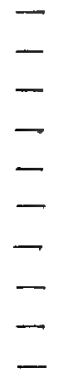

31 Celestine(8),

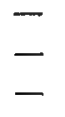

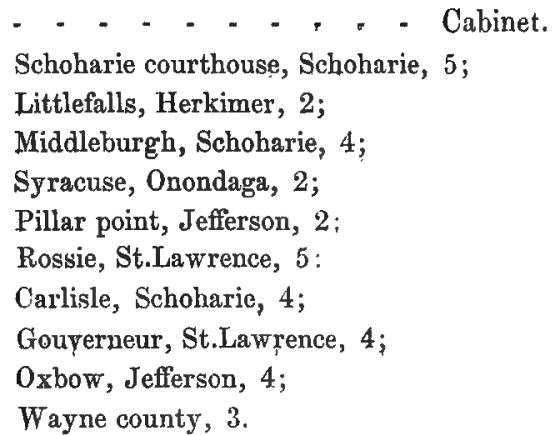

Schoharie courthouse, Schoharie, 5 ;

Littlefalls, Herkimer, 2;

Syracuse, Onondaga, 2;

Pillar point, Jefferson, 2

Rossie, St.Lawrence, 5:

Carlisle, Schoharie, 4;

Oxbow, Jefferson, 4;

Wayne county, 3 .

Stark, Herkimer, 3 ;

Syracuse, Onondaga, 2;

Lockport, Niagara, 2.

PRDẸR II. STRONTIAN,

32 Strontianitp $(12)$,

Schoharie courthouse, Schoharie, 11;

Antwerp, Jefferson, 1.

33 Barystrontanite, Near Hamilton College, Oneida.

ORDER III. LIME.

34 Calgarenous gapap(157),

Gouverneur, St.Lawrence, 5;

Diana, Lewis, 2;

Antwerp, Jefferson, 11;

Oxbow, Jefferson, 9;

Littlefalls, Herkimer, 3;

Middleville, Herkimer, 3;

Camillus, Onondaga, 3 ;

Haverstraw, Rockland, 5;

$-$

Warwick, Orange, 2;

Amity, Orange, 4; 
Names.

Calcareots spar :

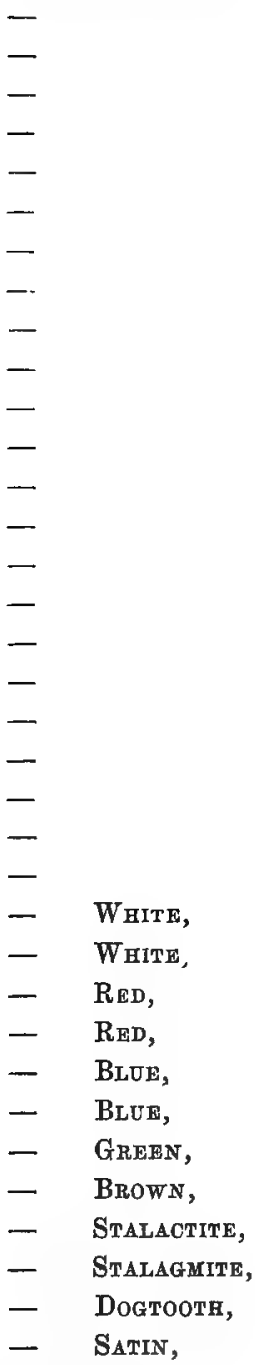

35 Marble(5),

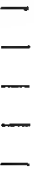

Localities.

Newburgh, Orange, 1;

Martinsburgh, Lewis, 4;

Rossie, St.Lawrence, 33;

Catskill, Greene, 11;

Canaan, Columbia, 1;

Middleburgh, Schoharie, 2;

Cobleskill, Schoharie, 3 :

Diamond point, Warren, 3;

Fort-Ann, Washington, 1;

Parish mine, St.Lawrence, 5;

Monroe, Orange, 2;

Port Henry, Essex, 9;

Ball's cave, Schoharie, 4;

Schoharie courthouse, Schoharie, 1;

Glenville, Schenectady, 1;

Rogersrock, Essex, 1;

Yonkers, Westchester, 1;

$O^{\prime}$ Niel mine, Orange, 1;

Muscolunge lake, Jefferson, 1;

Nethaway's cave, Schoharie, 1;

Young's cave, Schoharie, 1;

Caldwell, Warren, 1;

Lockport, Niagara, 2;

Catskill, Greene, 2;

Edenville, Orange, 1;

Cornwall, Orange, 4;

Monroe, Orange, 3;

Naturalbridge, Lewis, 1;

Diana, Lewis, 2;

Schroon, Essex, 1;

Flat creek, Montgomery, 1;

Ball's cave, Schoharie, $\mathbf{1}$;

Ball's cave, Schoharie, 1;

Cheshire, Ontario, 1;

Rossie, St.Lawrence, 1.

Montagne's quarry, Rockland, 1;

Hastings, Weștchester, 1;

Haverstraw, Rockland, 1;

Grassy point, Rockland, 1;

Pleasant-valley, Dutchess, 1. 
Naines.

Localities.

36 Fibrous Limestone(11),

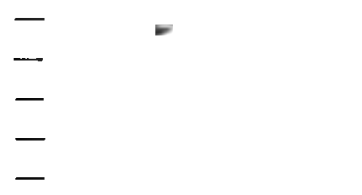

Camillus, Onondaga, 6;

Naturboridge, Jefferson, 1;

Ball's cave, Schoharie, 1;

Carlisle, Schoharie, 1;

Rossie, St.Lawrence, 2.

37 Concreted carbonate of lime(22), - - - - Cabinet.

- Agaric mineral, Cobleskill, Schoharie, 1;

- Oolitic limestone, Warwick, Orange, 2;

- Oolitic limestoxe, Greenfield, Saratoga, 2;

- Calcareous sixter, Newburgh, Orange, 1;

- Calcareous tufi, Jamesville, Onondaga, 4;

- Calcareous tufa, Lenox, Madison, 2;

- Calcareous tufi, Rochester, Monroe, 1;

- Calcareous tula, Syracuse, Onondara, 1;

- Calcareous tufa. Chittenango, Madison, 1;

- Calcareous tufa, Martinsburgh, Lewis, 1;

- Calcargous tufa, Marcellus, Onondaga, 1;

- Ferruginous, Johnsburgh, Warren, 3;

- Crystallizad, Anthony's Nose, Putnam, :

38 MARL(5),

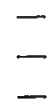

39 Arragonite(6),

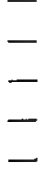

40 Grpsum(32):

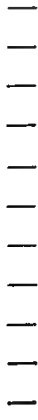

- -

Dewitt, Onondega, 1;

Syracuse, Onondaga, 3;

Salina, Onondaga, 1.

Monroe, Orange, 1;

Patterson, Putnam, 1;

O'Niel mine, Orange, 2;

Edenville, Orange, 1;

How's cave, Schoharie, 1.

Stark, Herkimer, 2; ,

Lockport, Niagara, 2;

Lenox, Madison, 1;

Onondaga county, 2;

Syracuse, Onondaga, 4;

Dewitt, Onondaga, 6;

Manlius, Onondaga, 4;

Camillus, Onondaga, 7 ;

Cayuga county, 1;

Lockport, Niagara, 1;

0 'Niel mine, Orange, 1. 
Nanes.

41 AnHydrite,

42 Apatite(13),

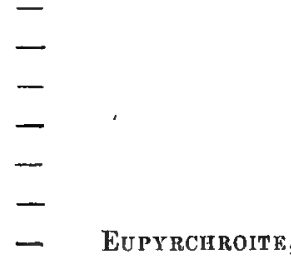

43 Fldor spar(15),

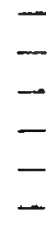

44 Muriate of lime,

45 Datholite,

46 Pharmacolite,
Localities.

Lockport, Niagara.

Rossie, St.Lawrence, 1;

Hammond, St.Law rence, 1;

Hall mine, Essex, 3;

Edenville, Orange, 4;

Westfarms, Westchester, 1;

Diana, Lewis, 2;

Crownpoint, Essex, 3.
Cabinet.

Lowville, Lewis, 2;

Amity, Orange, 2;

Niagara, Niagara, 4;

Schoharie courthouse, Schoharie, 3 ;

Alexandria, Jefferson, 2;

Manlius, Onorldiga, 2.

Water of several mineral springs:

Piermont, Rockland, etc.

Monroe, Orange.

ORDER IV. MAGNESIA.

47 BRUCITE,

Quarantine, Richınoud, etc.

48 Carbonate of Magnesia, Staten island, etc.

49 EPsom salt,

Bethlehem, Albany, etc.

50 Magnesian carbonate of lime(27), - - - - - Cabinet.
- Pearl spar,
Lockport, Niagara, 1;
- Pearl spar,
Littlefalls, Herkimer, 2;
- Pearl spar,
Niagara falls, Niagara, 2;
- Pearl spar,
Rossie, St.Lawrence, 4;
- Pearl spar,
Theresa, Jefferson, 1;
- Brown SPAR,
Diamond island, Warren, 2;
- BrowN SPAR,
Parish mine; St.Lawrence, 3 ;
- Dolomite,
Kingsbridge, New-York, 2;
- Dolomite,
Amenia, Dutchess, 1;
- Gurhofite,
Phillipstown, Putnam, 5;
- Gurhofte,
Quarantine, Richmond, 4.

51 Hydraulic limestone(3), -

Schoharie courthouse, Schoharie, 1;

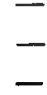

Van Eps's quarry, Montgomery, 1;

Lockport, Niagara, 1.

52 Hydrobromate of magnesia, In sea.water, otc. 


\section{CLASS VI. EARTHY MINERALS.}

Names.

53 QUaRtz(65),

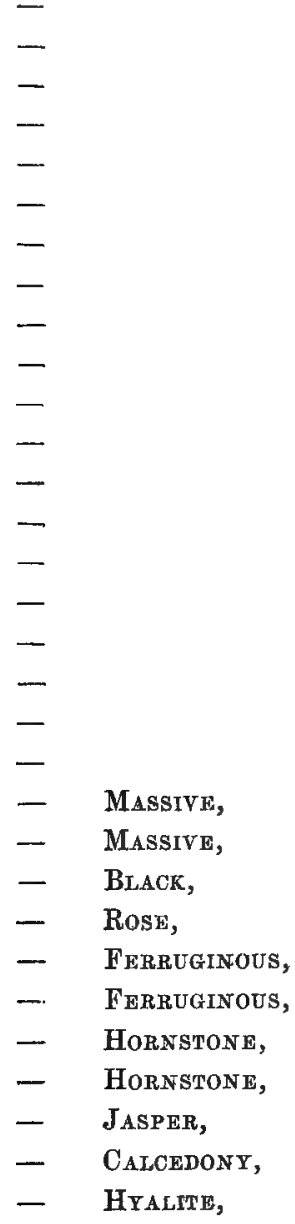

54 Tabular spar(7),

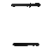

55 Nemalite,
ORDER I. SILICA.

Loealities.

Middleville, Herkimer, 2;

Littlefalls, Herkimer, 1;

Port Richmond, Richmond, 3 ;

Diamond point, Warren, 3 ;

Craigville, Orange, 2;

Ellenville, Ulster, 3;

Oxbow, Jefferson, 2;

Redbridge, Ulster, 4;

Sterling mine, Jefferson, 2;

Diana, Lewis, 4;

Wurtzborough mine, Sullivan, 2;

Catskill, Greene, 1;

Ticonderoga, Essex, 1;

Rossie, St.Lawrence, 3;

Root, Montgomery, 2;

Shawangunk mountains, Sullivan, 1;

Saratoga springs, Saratoga, 2;

Warwick, Orange, 3;

Hastings, Westchester, 1;

Leyden, Lewis, 1;

New-Rochelle, Westchester, 1;

Yonkers, Westchester, 3 ;

Littlefalls, Herkimer, 2;

Port Henry, Essex, 3;

Quarantine, Richmond, 2;

Warwick, Orange, 1;

New-Rochelle, Westchester, 1;

Sprakers, Montgomery, 2;

Warwick, Orange, 2;

Warwick, Orange, 3 ;

Phillips's mine, Putnam, 2.

Lewis, Essex, 6;

Diana, Lewis, 1.

Piermont, Rockland. 
Names.

56 Serpentine $(107)$,

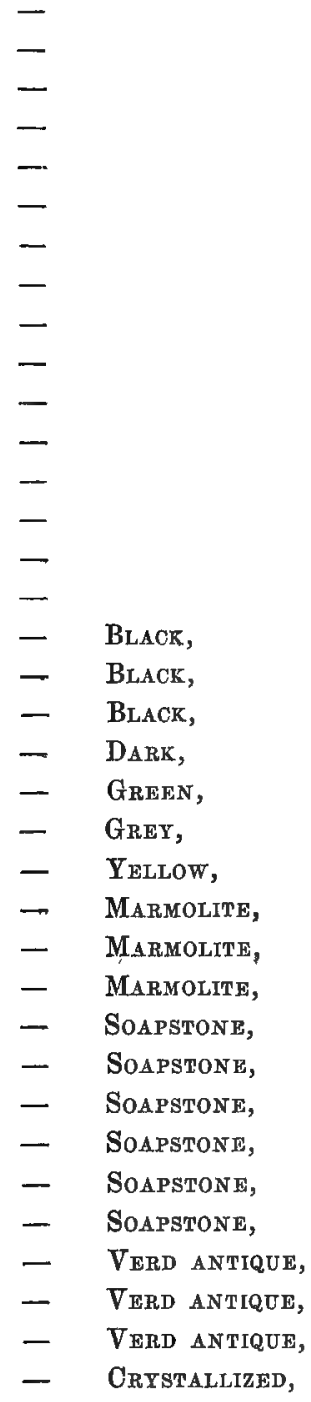

57 Magnestte(4),

58 Chondrodite(37),
Localities.

Syracuse, Onondaga, 8;

New-Rochelle, Westchester, 8;

Rye, Westchester, 3;

Monroe, Orange, 1;

Rossie, St.Lawrence, 4;

Port Henry, Essex, 2;

Phillipstown, Putnam, 4;

New.York, New-York, 2;

Diana, Lewis, 2;

Quarantine, Richmond, 2;

Gouverneur, St.Lawrence, 3;

Antwerp, Jefferson, 1;

Warwick, Orange, 1:

Amity, Orange, 2;

Oxbow, Jefferson, 5;

Natural-dam, St.Lawrence, 2;

Warwick, Orange, 2;

Forshee mine, Monroe, Orange, 2;

Southeast, Putnam, 1;

Brown's quarry, Putnam, 3;

Fort-Ann, Washington, 4:

Fort-Ann, Washington, 2;

Phillipstown, Putnam, 4;

New-Rochelle, Westchester, 1;

Rye, Westchester, 1;

Phillipstown, Putnam, 2;

Warwick, Orange, 3;

Fort-Ann, Washington, 1;

Wilks mine, Monroe, Orange, 1;

Amity, Orange, 1;

Diana, Lewis, 1;

Gouverneur, St.Lawrence, 2;

Port Henry, Essex, 5;

Amity, Orange, 6;

Gouverneur, St.Lawrence, 2;

Greenwood, Orange, 1.

Stony point, Rookland. - - Cabinet.

Southeast, Putnam, 1;

Port Henry, Essex, 3;

Rossie, St.Lawrence, 8;

Amity, Orange, 12; 
Names.

Chondrodite :

59 Boltonite,

60 Tale(12),

61 Pyroxene(72),

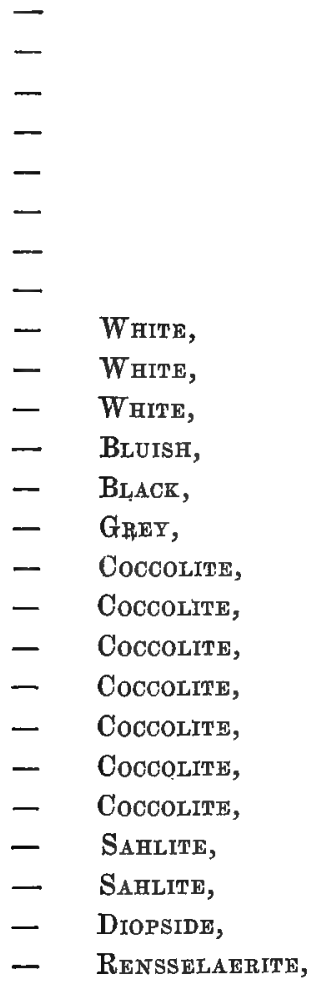

62 Hornblende(194),

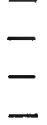

Loculities.

Edenville, Orange, 5;

Naturalbridge, Orange, 2;

Monroe, Orange, 3;

Edenville, Orange, 2;

Monroe, Orange, 1.

Several places in Orange county.

Quarantine, Richmond, 3;

Amity, Orange, 5;

Fishkill, Dutchess, 4.

Edenville, Orange, 6;

O'Niel mine, Monroe, Orange, 1;

Rogersrock, Essex, 2;

Ticonderoga, Essex, 4;

Greenwood, Monroe, Orange, 4;

Warwick, Orange, 3;

Gouverneur, St.Lawrence, 2;

Rossie, St.Lawrence, 2;

Yonkers, Westchester, 1;

Patterson, Putnam, 3;

Kingsbridge, New-York, 2;

Haverstraw, Rockland, 2;

Crag harbor, Essex, 1;

Richville, St.Lawrence, 2;

Monroe, Orange, 5;

Rogersrock, Essex, 2;

Lewis corners, Essex, 2;

Phillipstown, Putnam, 2;

Diana, Lewis, 2;

Carmel, Putnam, 1;

Port Henry, Essex, 1;

Monroe, Orange, 5;

Moriah, Essex, 1;

Phillipstown, Putnam, 1;

Oxbow, Jefferson, 2.
Cabinet. 
Names.

Hornblende :

\begin{tabular}{|c|c|}
\hline \multicolumn{2}{|l|}{ - } \\
\hline \multicolumn{2}{|l|}{ - } \\
\hline \multicolumn{2}{|l|}{-} \\
\hline \multicolumn{2}{|c|}{-} \\
\hline \multicolumn{2}{|l|}{ - } \\
\hline$\rightarrow$ & \\
\hline \multicolumn{2}{|l|}{-} \\
\hline - & 冫 \\
\hline \multicolumn{2}{|l|}{-} \\
\hline - & BowIDER, \\
\hline - & BLACK, \\
\hline 一 & BLACK, \\
\hline 一 & BLACK, \\
\hline - & GREY, \\
\hline- & Brown, \\
\hline - & GRANULAh, \\
\hline - & Pargasite, \\
\hline - & Pargasitg, \\
\hline - & EDENITE, \\
\hline- & ACTINOLITE, \\
\hline$\longrightarrow$ & ACTINOLTTE, \\
\hline$\tau$ & Actinolite, \\
\hline- & ACtinolite, \\
\hline- & Actinolite, \\
\hline- & AsBestus, \\
\hline & AsbeSTUS, \\
\hline- & ÁSBESTUS, \\
\hline- & AsBESTUS, \\
\hline- & AsBestus, \\
\hline- & Asbestus, \\
\hline- & Asbestus, \\
\hline- & Tremolite, \\
\hline- & Tremolite, \\
\hline - & do. \\
\hline- & do. \\
\hline - & do. \\
\hline- & do. \\
\hline- & do. \\
\hline & do. \\
\hline & $\begin{array}{l}\text { do } \\
\text { do. }\end{array}$ \\
\hline & do. \\
\hline & do. \\
\hline
\end{tabular}

r,ocalities.

Peckville, Fishkill, Dutchess, I; Yonkers, Westchester, 3;

Sdenville, Orange, 13;

Warwick, Orange, 8;

Southeast, Putnam, 14;

Gouverneur, St.Lawrence, 2;

Stony point, Rockland, 1;

Florida, Orange, 4;

Moriah, Essex, 5;

Piermont, Rockland, 2;

Nyack, Rockland, 1;

New-Rochelle, Westchester, 3;

Ticonderoga, Essex, 2;

Crag harbor, Essex, 1;

Monroe, Orange, 4;

Amity, Orange, 4;

Crag harbor, Essex, 2;

Whitehall, Washington, 2;

Rossie, St.Lawrence, 1;

Warwick, Orange, 3 ;

Cornwal, Orange, 1;

Fishkill, Dutchess, 2;

Rye, Westchester, 1;

Grassy point, Rockland, 1;

Kent, Putnam, 2;

Quarantine, Richmond, 4;

Stony point, Rockland, 1;

Monroe, Orange, 6;

Patterson, Putnam, 2;

Newburgh, Orange, 3;

Phillipstown, Putnam, 4;

Oxbow, Jefferson, 1;

Patterson, Putnam, 4;

Gouverneur, St.Lawrence, 2;

Richville, St.Lawrence, 3;

Hastings, Westchester, 6;

Dover, Dutchess, 3 ;

Edenville, Orange, 2;

Kingsbridge, New-York, 4;

Amity, Orange, 4;

Phillipstown, Putnam, 3;

Port Henry, Essex, 2;

New-Rochelle, Westchester, 3;

Lessie, St.Lawrence, 3; 
Names.

Localities.

\section{Hornblende :}

- Pseudomorphs,
- Pshudomorphs,

63 Arfwedsonite? (1)

64 Hypersteene(1),
Edenville, Orange, 2;

Amity, Orange, 3.

Carmel, Putnam. - - - Cabinet.

Putnam, Washington. - - Cabinet.

65 Schiller spar, Carmel in Putnam, and Amity in Orange.

66 ANThophyLLite(5),

Rye, Westchester, 2;

New-Rochelle, Westchester, 3.

67 Hydrous anthophyltite(5), - - a - - - Cabiuet.

$\overline{-}$

68 Corundum,

69 Spinellet $(40)$,

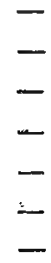

70 Aбтомоlite,

71 Gibbsite(1),

72 Aluminitie,

73 IDOCRAsE(6),

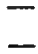

74 GarNet(50),

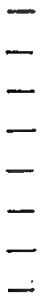

New-York island, 2;

New-Rochelle, Westehester, 2;

Fishkill, Dutchess, 1.
ORDER II. ALUMINA.

Near Amity, Orange.

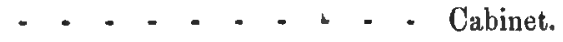

Amity, Orange, 19;

Edenville, Orange, 10;

Mount Eve, Orange, 2;

Warwick, Orange, 3;

Oxbow, Jefferson, 4;

Gouverneur, St.Lawrence, 1;

Rossie, St.Lawrence, 1.

Rossie, St.Lawrence.

Unionvale, Dutehess.

In the clay of Staten island, etc.

Amity, Orange: 4;

Antwerp, Jefferson, 2.

Amity, Orange, 3;

Rogetsrock, Essex, 12;

Greenfield, Saratoga; 1;

Lewis, Essex, 5;

New-Rochelle, Westchester, 2;

Hague, Warren, 1;

Sprakers, Montgomery, 4;

Westfarms, Westchester, ]; 
Names.

GARNET :
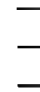

- Colophonite,

- Colophonite,

- Colophonite,

- Colophonite,

- Cinnamon-stonir,

75 Scapolite(30),

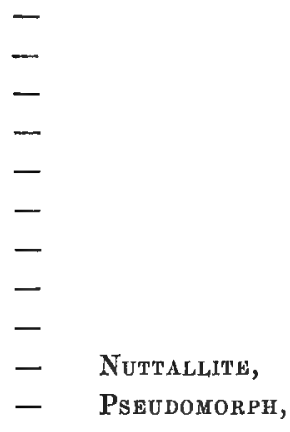

76 Staurolite(1),

77 FeLdSPar(46), 
Names.

78 Labradorite(8),

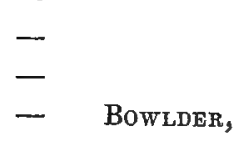

79 Albite(1),

80 STELLite(4),

81 Mesotype,

82 Stilbite(9),

83 Heulandite,

84 Epistilbite,

85 Apophyllite,

86 Prehnite(1),

87 Thomsonite,

88 LaUmonite,

89 Analcime,

90 Chabazite,

91 Eтidote(15),

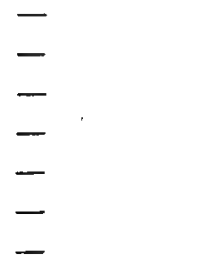

92 Todrmaline(25),

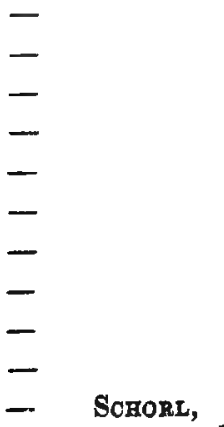

Localities.

Lewis corners, Essex, 2;

Moriah, Essex, 4;

Ogdensburgh, St.Lawrence, 1;

Hamptonburgh, Orange, 1.

Granville, Washington. - - Cabinet.

Piermont, Rockland. - - Cabinet.

Harlem, New-York.

Harlem, New-York, 5;

Peekskill, Westchester, 4.

Near Westfarms, Westchester.

In Rockland county?

Harlem, New-York.

Westport, Essex. - - - C Cabinet.

Piermont, Rockland.

Phillipstown, Putnam (exhausted).

Near Yonkers, Westchester.

Piermont, Rockland.

Harlem, New-York, 4j

Carmel, Putnam, 2;

Cornwal, Orange, 1;

Wạwick, Orange, 3 ;

Ticonderoga, Essex, 2;

Westport, Essex, 2;

Putnam, Washington, 1.

Port Henry, Essex, 2;

Gouverneur, St.Lawrence, 1;

Yonkers, Westchester, 2;

Harlem, New-York, 2;

Edenxille, Orange, 3;

W.ambick, Orange, 2;

Amity, Orange, 2;

Greenfield, Saratoga, 1;

Richfield, St.Lawręnce, 1;

Ticonderoga, Essex, 1;

Essex county, 1.

Cabinet. 
Names.

93 Clintonite(2),

94 Andalusite,

95. Bucholzite(5),

96 Kyanite,

97 Aснмiте,

98 Pitchitone,

99 Spodumene,

100 Pyrophyllite,

$101 \operatorname{Mica}(73)$,

-

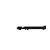

$-$

- Black,

- YelLOW,

- GREEN,

- GREeN,

- SILVERY,

- Silvery,

- Copper,

- Lithion?

- Orystalized,

Localities.

Amity, Orange. - - - - Cabinet.

Near Nyack, Rockland.

Cornwal, Orange, 1;

Monroe, Orange, 3.

Near New-York city.

Lockport, Niagara.

Dykes in Essex and Warren.

Near Saratoga springs,

Near Westpoint, Orange.

Ticonderoga, Essex, 1;

Antwerp, Jefferson, 5;

Oxbow, Jefferson, 2;

Rossie, St.Lawrence, 2;

Edwards, St.Lawrence, 2;

Gouverneur, St.Lawrence, 2;

Diana, Lewis, 1;

Greenfield, 'Saratoga, 5;

Putnam, Washington, 1;

Monroe, Orange, 12;

Edenville, Orange, 8;

Amity, Orange, 1;

Warwick, Orange, 4;

Kane's quarry, Westchester, 2;

Westfarms, Westchester, 1;

New-Rochelle, Westchester, 2;

Rye, Westchester, 1;

Southeast, Putnam, 1;

Yonkers, Westchester, 1;

Warwick, Orange, 1;

Greenwood, Monroe, Orange, 1;

Yonkers, Westchester, 4;

Edwards, St.Lawrence, 2;

Amity, Orange, 1;

Edenville, Orange, 3;

Edenville, Orange, 2;

Antwerp, Jefferson, 5.

Cabinet. 


\section{ORDER III. GLUCINA.}

Names.

Localities.

102 Emeraid, Near New-York city, and near Singsing in Westchester. 103 Chrysoberyu(2),

Greenficld, Saratoga. - - Cabinet.

ORDER IV. ZIRCONIA.

104 Zirconite(18),

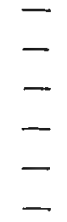

Warwick, Orange, 8;

Edenville, Orange, 1;

Cornwal, Orange, 3 ;

Canterbury, Orange, 1;

Delong's mill, St.Lawrence, 1;

Hall mine, Moriah, Essex, 2.

\section{CLASS VII. METALLIC MINERALS.}

\section{ORDER I. IRON.}

105 Native IRON,

Near Penn-Yan, Yates?

106 Magnetic IRON ORE(83),

Moriah, Essex, 15;

Cabinet.

Ticonderoga, Essex, 2;

Crownpoint, Essex, 3;

Crag harbor, Essex, 2;

Clinton county, 5;

Antwerp, Jefferson, 1;

French mountain, Warren, 2;

Jessup's landing, Saratoga, 2;

Salisbury, Herkimer, 2;

Mouroe, Orange, 37;

Cornwal, Orange, 6;

Canterbury, Orange, 1;

Phillipstown, Putnam, 4;

Southeast, Putnam, 3;

- IRON SAND,

Haverstraw, Rockland, 2;

- IRON SAND,

Port Henry, Essex, 2;

- Iron SAND,

Duck pond, Suffolk, 1;

- IRON SAND,

Lyonsdale, Lewis, 1;

- IRON SAND,

Diana, Lewis, 1;

Gouverneur, St.Law rence, 1. 
Names.

107 Specular iron ore(25),

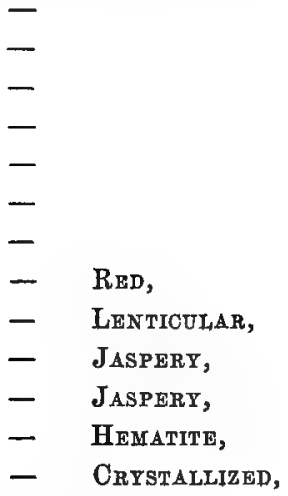

108 Limonite(36),

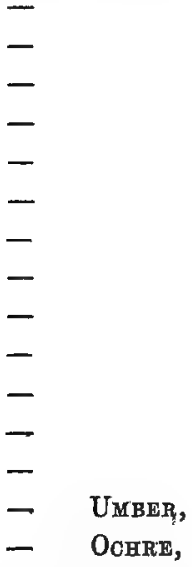

109 Iron pyrites(20),

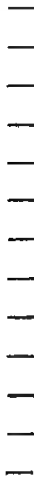

SUlPHURET,

Iocalities.

Hermon, St.Lawrence, 6;

Gouverneur, St.Lawrence, 2;

Edwards, St.Lawrence, 3;

Antwerp, Jefferson, 2;

Theresa, Jefferson, 2;

Naturalbridge, Jefferson, 2;

Port Henry, Essex, 1;

Oxbow, Jefferson, 1;

Rochester, Monroe, 2;

Hall mine, Moriah, Essex, 1;

Parish mine, St.Lawrence, 1 ;

Ticonderoga, Essex, 1;

Gouverneur, St.Lawrence, 1.

Oxbow, Jefforson, 2;

Port Henry, Essex, 1;

Whitehall, Washington, 2;

Fishkill, Dutchess, 2;

Unionvale, Dutchess, 5;

Amenia, Dutchess, 1;

Monroe, Orange, 7 ;

Near Canterbury, Orạnge, 3 ;

Warwick, Orange, 1;

Edenville, Orange, 2;

Wurtzborough, Sullivan, 1;

Near Quarantine, Richmond, 3;

Peekskill, Westchester, 2;

Monroe, Orange, 3;

Greig, Lewis, 2.

- Cabinet.

- - - - - - . C Cabinet.

Rossie, St.Lawrence, 1;

Port Henry, Essex, 2;

Diana, Lewis, 2;

Martinsburgh, Lewis, 3;

Flat creek, Montgomery, 1;

Yonkers, Westchester, 1;

'Schoharie courthouse, Schoharie, 2;

Kingsbridge, New-York, 1;

Wurtzborough, Sullivan, 1;

Newburgh, Orange, 2;

Warwick, Qrange, 1;

Theresa, Jefferson, 1;

Gouverneur, St.Lawrence, 2. 
Names.

Localities.

110 Magnetic IRON PYRITES(3),

Monroe, Orange, 1;

- $\quad$ Port Henry, Essex, 2.

111 White IRoN pYRITES, Near Warwick in Orange, etc.

112 Arsenical iron pyrites(10), - - - - - - Cabinet.

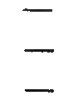

113 SCORODITE,

114 Iron SINTER(3),

115 StRthose IRON(5),

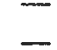

116 ANKERITE,

117 Copperas,

118 Alumina-sulphate of IRon, Rossville, Richmond.

119 Chrome-Iron ore(2),

120 Vivianite,

121 CaCoXenite(2),

122 Ilvaite,

123 Silicate of IRON,

124 Hudsonite(1),
Brown's quarry, Putnam, 3;

Edenville, Orange, 6;

Amity, Orange, 1.

Warwick, Orange.

Edenville, Orange.

Parish mine, St.Lawrence, 4;

Dodge mine, Hermon, St.Lawrence, 1.

Johnsburgh, Warren.

Not referred to.

-

Quarantine, Richmond, 1;

Rye, Westchester, 1.

Harlem, New-York.

Sterling mine, Antwerp, Jefferson. Cabinet. In Essex county?

Ramapo, Rockland.

Cornwál, Orange. - - Cabinet.

ORDER 1I. MANGATANESE.

125 Oxide of Manganeise(5), - - - - - - - - Cabinet.

Unionvale, Dutchess, 1;

Warwick, Orange, 2;

-

Sprakers, Montgomery, 1;

126 MANGANESE SPAR, Crownpoint, Essex, 1.

127 Babingtoniter,

In Essex and Warren counties.

Gouverneur, St.Lawrence.

ORDER III. ZINC.

128 ZINC BLENDE(10), 
Names,

Localities.

ZINO BLENDE :

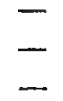

Wurtzborough, Sullivan, 4;

Lockport, Niagara, 2;

Niagara falls, Niagara, 1 .

ORDER IV. LEAD:

129 Galena(22),

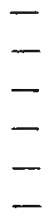

- - - - - - - - Cabinet.

Old Rossie mine, St.Lawrence, 2;

Mineral point, St.Lawrence, 3;

Martinsburgh, Lewis, 8;

Flat creek, Root, Montgomery, 3;

Wurtzborough, Sullivan, 5;

Macomb, St.Lawrence, 1.

130 WHITE LEAD ORE, In St.Lawrence and Westchester counties.

131 Anglesite,

132 YELLOW LEAD ORE,

133 Prromorphite,

134 VAUQUelintte,
Rossie, St.Lawrence.

Aneram, Columbia.

Near Singsing, Westchester.

Near Singsing, Westchester:

ORDER V. BISMUTH.

135 Native bismuth,

In Essex county?

ORDER VI. COPPER.

136 NATIVE COPPER,

137 RED COPPER ORE,

138 VITREOUS COPPER,

139 Copper Pyrites(6),

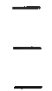

140 Green malachite(2),

141 AzURIte,
In Washington county?

Ladenton, Rockland.

In Columbia and Dutchess counties.

- . . - . . . . Cabinet.

Rossie, St.Lawrence, 1;

Wurtzborough, Sullivan, 1;

Kane's quarry, Westchester, 1.

Phillips mine, Putnam, 1;

Theresa, Jefferson, 1.

Singsing, Westchester.

ORDER VII. TITANIUM.

142 Native titanidm,

143 Anatase(1),
Furnaces of St.Lawrence county, etc.

Phillipstown, Putnam. - - Cabinet. 
Names.

144 RUTiLe(2),

145 Ilmenite(7),

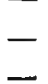

146 Sphene(17),

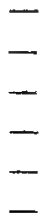

147 WARWICKITE(4),

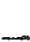

Localities.

Amity, Orange. - - - Cabinet. - - - - - . - Cabinet.

Warwick, Orange, 4;

Stirling mine, Monroe, Orange, 2;

Cornwal, Orange, 1.

Diana, Lewis, 2;

Phillips mine, Putnam, 2;

Warwick, Orange, 2;

Amity, Orange, 2;

Bush mine, Cornwal, Orange, 2;

Gouverneur, St.Lawrence, 3.

Warwick, Orange, 2;

Edenville, Orange, 2.

ORDER VIII. MOLYBDENUM.

148 Molybdenite(1),

23d-street, New-York city. - Cabinet.

ORDER IX: ARSENIC:

149 ORPIMENT,

Near Carmel, Putnam.

ORDER X. OERIUM.

150 Allanite,

Monroe, Orange:

ORDER XI. SILVER.

151 Native silver,

Singsing, Westchester.

152 Vitreous silver,

Livingston's mine, Columbia. 


\section{A D D I T I O N S.}

MINERALS FOUND SINCE THE MINERALOGY WAS PUBLISHED.

Names.

153 Terenite,

154 Columbite,

155 Loxoclase,

156 Monazite,

157 NitRate OF LiME,

158 Phyllite,

159 YTTRO-OERITE,

160 Dysintribite(1),

161 HodGhite(3),

162 Millerite(2),
Localities.

Antwerp, Jefferson.

Greenfield, Saratoga.

Rossie, St.Lawrence.

Yorktown, Westchester.

Marbletown, Ulster.

Clove iron mine, Dutchess.

Amity, Orange.

Theresa, Jefferson. - - - - Cabinet.

Gouverneur, St.Lawrence. - Cabinet.

Antwerp, Jefferson. - - - Cabinet. 


\section{GEOLOGY OF NEW-Y0RK,}

BY

WILLIAM W. MATHER, EBENEZER EMMONS, LARDNER

VANUXEM, AND JAMES HALL. 



\section{GE0L0GY.}

[All the specimens enumerated under this division of the Catalogue are in the Cabinet, unless specially cited as missing.]

\section{CRYSTALLINE AND METAMORPHIC ROCKS OF THE ANTE-SILURIAN PERIOD.}

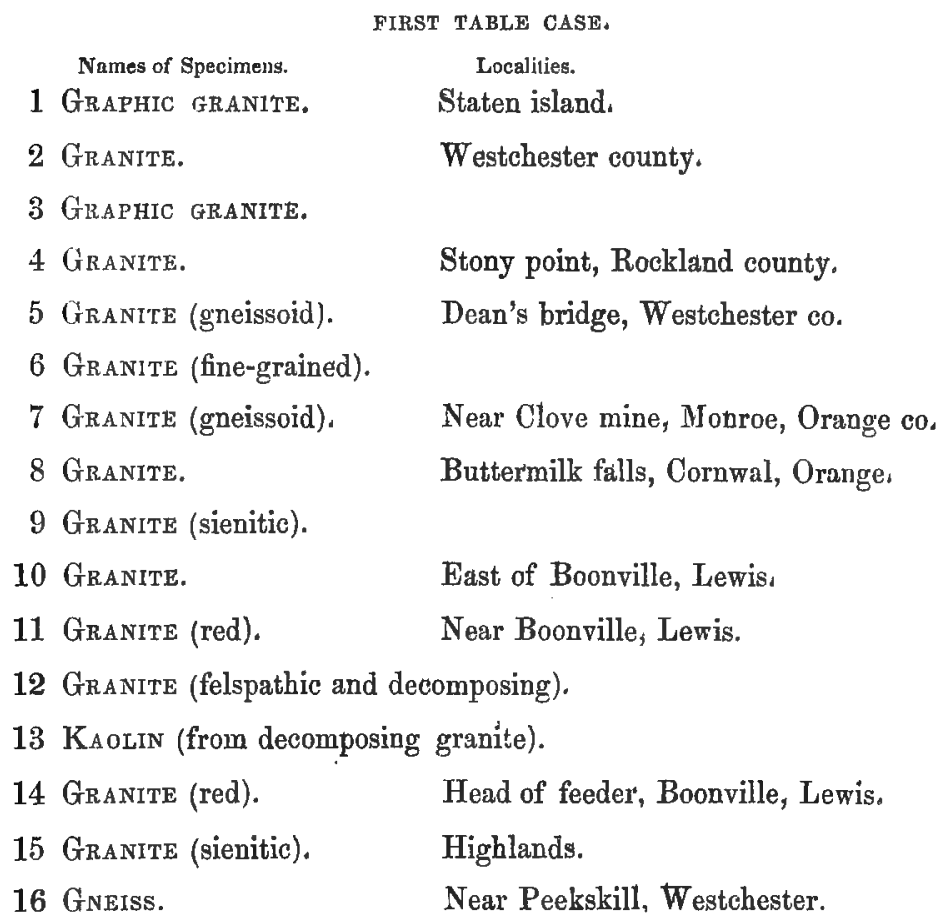


Names

$17 \& 18$ Gneiss.

19 GNEISS.
Loculities.

Near Coldspring, Putnam.

Above Tarrytown, Westchester.

20 GNEIss (with calcareous matter). Littlefalls.

$21 \& 22$ GNEISS.

23 Gneiss.

24 GNEISS.

25 GNEISS.

$26 \& 27$ GNeiss.

28 GNeiss.

29 Gineiss.

30 GNEISS.

31 GNEIss (epidotic).

32 Hornblende.
Littlefalls.

Two miles south of Cranberry creek.

South of Westpoint.

Northeast of Fourth lake, Hamilton.

Trenton village, near Remsen.

Between Kingsborough and Clarke's.

Salisbury, Herkimer.

Yorktown, Westchester.

Near New-Rochelle, Westchester.

33 - 36 Magnetic oxide of ikon. Monroe, Orange.

$37 \& 38$ Hypersthene rock. Moriah, Essex.

39 Red gRanite.

Lewisburgh, Iuewis.

40 Blue crystaline linestone. Naturalbridge, Jefferson.

41 Crystalime linestone. Northeust of Fourth lake, Hamilton.

42 Micaceous limestone. Northeast of Lewisburgh, Lewis.

43 Crxstaldine limestone (with quartz and augite). N.F. Fourth luke.

44 Crystalime limestone. Northeast of Lewisburgh, Lewis.

45 Fused quartz. Rossie, St.Lawrence.

46 Quartz and Mica.

Northeast of Fourth lake, Hamilton.

47 Coccolite.

Northeast of Fourtl lake, Hamilton.

48 Purple scapolite. Antwerp, Jefferson.

49 Iron ORE. Alexandria, Jeffersou.

50 Specular iron ore.

Edwards, St.Lawrence.

51 Specular oxide of iron (with quartz crystals). Fowler, St.Lawrence.

52 Specular oxide of Iron (with quartz and limestone). Edwards.

53 Peroxide of iron (with cacoxene). Sterling nine, Jefferson.

54 Sulphate of strontian. 
ROOKS.

Names.

Localities.

$56 \& 57$ Talc.

Fowler, St.Lawrence.

$58 \& 59$ TALC.

Naturalbridge, Jefferson.

60 Rensselaerite (with satin spar). Fowler, St.Lawrence.

61 White talc. Fowler, St.Lawrence.

(j2) Cacoxene (on gneissoid rock). Naturalbridge, Jefferson.

63 SCapolite RocK.

In northeast part of Lewis county.

64 Crystalline Limestone (with brucite). Schroon, Essex.

FIRST WALL CASE.

65 - 67 Laвкаронits. Adirondack, Essex.

68 Labradorite (sienitic). Adirondack, Essex.

69 Hypersthene rock (sienitic). Moriah, Essex.

70 LaBRADORITE.

Mayfield corners, Montgomery.

71 Hypersthene rock. Port Kent, Essex.

72 Porphyritic talcose slate. Westport, Essex.

73 Graphic granite. Warwick, Orange.

74 Granite in limestone. Warwick, Orange.

75 Granite.

Long mine, Monroe, Urange.

76 Granitic Rock.

Mountain mine, Monroe, Orange.

77 Granite.

Royahook, Westchester.

78 Granite.

Saw-works, Monroe, Orange.

79 Granite.

Patterson la..., Monroe, Orange.

80 Granite (sienite).

Near Fort Muritgomery, Orange.

81 Granite.

Tenth avenue, New-York city.

82 Felspathic granite. Tarrytown, Westchester.

83 Gneiss (with magnetic oxide of iron). Shenadone, Dutchess.

84 GNeIss (junction of hypersthene rock).

85 Gneiss (with garnet). $\quad 0^{\prime} \mathrm{Niel}$ mine, Orange.

86 GNeiss.

Fort Montgomery, Orange.

87 GNEISS.

Coldspring, Putnam.

88 Gneiss.

Carmel, Putnam.

$89 \& 90$ GNEISS.

Near Singsing, Westchester.

91 - 93 GNeISS.

Near Westpoint, Orange. 
NEW-YORK STATE COLLECTION.

Names.

94 Greiss.

95 GNeiss.

96 Gneiss.

97 GNEISS.

98 GNEISS.

99 GNeIss.
Loculities.

- Near Dobbs's ferry, Westchester.

Royahook, Westchester.

Mountpleasant, Westchester.

Near Westpoint, Orange.

Stormville, Dutchess.

Somerstown plains, Westchester.

100 - 102 Hypersthene rock passing into gneiss. Moriah, Essex.

103 Gneiss.

$104 \& 105$ Gneiss.

106 Mica slate.
Shenadone, Dutchess.

Herkimer county.

Whiteplains, Westchester.

107 Mica slate (with staurotide). Hurd's corners, Dutchess.

108 Mica.

Clove mine, Monroe, Orange.

109 Sienite (primitive greenstone). Cortland, Westchester.

110 Sienite.

111 Hornblende.

112 Hornblende.

113 KaOLIN.
Moriah, Essex.

Warwick, Orange.

Westport, Essex.

114 Kaolis (or porcelain clay). Johnsburgh, Warren.

115 FELSPar.

Westport, Essex.

116 Sahlite (in hypersthene). Westport, Essex.

117 Scapolite Rock.

Ausable, Essex.

118 Magnetic oxide of iron. Sanford ore bed, Essex.

119 \& 120 HLagnetic oxide of iron. Salisbury, Herkimer.

121 Magnetic oxide (in limestone). Near Coldspring, Putnam.

122 - 125 Magnetic oxide or iron. Monroe, Orange.

126 Magnetic oxide (with arragonite). O'Niel mine, Monroe, Orange.

127 Brack oxide of manganese. Crownpoint, Essex.

128 Copper pyrites (in limestone). Paradox lake, Essex.

129 ROSE QUARTZ.

130 White aUgite.

131. EptDote.

132 EPIDote (boulder).

133 Obsidian.
Moriah, Essex.

Near Phillipstown, Putnam.

Westport, Fissex.

In place in Vermont.

Johnsburgh, Warren. 
Names.

134 CoLophonlte.

Localities.

Johnsburgh, Warren.

135 Crystaline limestone. Port Henry, Essex.

136 Crystaldine limestone (with an unknown mineral). Port Henry.

137 Cristalinime limestone (with graphite). Port Henry, Essex.

$138 \& 139$ Crystaliine limestone (ferruginous). Johnsburgh, Warren.

140 Crystaline limestone (with mica and brucite). Schroon, Essex.

141 Crystalling limestone (with graphite).

142 Crystalime limestone (with quartz and pyroxene). Moriah, Fissex.

143 Crystalijne limestone (with serpentine). Bolton, Warren.

144 - 147 Crystaldine limestone (with serpentine). Moriah, Essex.

148 Crxstaldjne himestone (with serpentine). Port Henry, Essex.

149 Serpentine. Warrensburgh, Warren.

150 Serpentine and limestone. 58th-street, New-York city.

151 Limestone with brucite, Near Putnam courthouse, Putnam.

152 Crystalline linestone (with mica).

153 Crystalline juimestone. Near Putnam courthouse, Putnam. 154 - 156 Crystaline limestone (flesh-colored). Monroe, Orange.

157 Crystalline limestone (white).

158 White limestone. Edenville, Orange.

159 White limestone (with plumbago). Monroe, Orange.

160 Pyroxene or augite. Twoponds, Monroe, Orange.

161 Limestone (with augite and crystallized iron ore). Monroe, Orange.

162 Limestone (with mica). Monroe, Orange.

163 Limestone (with pargasite and meionite). Monroe, Orange,

164 Limestone (with pargasite), Island pond, Monroe, Orange.

165 Limestoxs (with serpentine or boltonite), Monroe, Orange.

166 Crystalline limestuil. Lear Westfarms, Westchester.

167 Granular imestone. Near Somerstown plains, Westchester,

168 Crystalline limestone. North-Salem, Westchester.

169 Limestone (with tremolite). Near North-Salem, Westchester.

170 Serpentine.

Forshee's mine, Monroe, Orange.

171 Serpentine (black).

172 Árpentine (with amianthus), O'Niel mine, Monroe, Orange: 
NEW-YORK STATE COLLECTION.

Names.

Localities.

173 Serpentine (striated surface).

174 Primitive gremnstone. Near Peekskill, Westchester.

175 \& 176 Dendritic porphyry. Essex, Essex.

177 Trap and himestone. Near Saw-works, Monroe.

178 Trap (striated surface). Near Ross's bridge, Essex.

179 VEINSTONE.

Near Singsing, Westchester.

180 Trap.

181 Trap.

Near Fort-Ann, Washington.

182 Trap

Near Saw-works, Monroe, Orange.

183 Trap.

Near Greenwood furnace, Monroe, Orange.

184 Trap (vesicular).

Parish ore bed, St.Lawrence.

185 Augite.

Sparta, Westchester.

\section{SECOND WALL CASE.}

186 Calcareous spar. Rossie lead mine, St.Lawrence.

187 Catcareous spar (purple). Oxbow, Jefferson.

188 Calcareous spar (with chalcedeny). Fowler, St.Lawrence.

189 Crystalline limestone.

190 Quartz crystals (in limestone). Laidlaw lake, Oxbow, Jefierson.

191 Granite (graphic). Gouverneur, St.Isawrence.

192 FELSPAR and TRAP. Alexandria, Jefferson.

193 GNEIss. Lewisburgh furnace, Lewis.

194 GNeIss. Rossie lead mine, St.Lawrence.

195 Mica slate. Northeast of Fourth lake, Hamilton.

$196 \& 197$ Crystalline limestone. Lewisburgh, Lewis.

198 Crystalline limestone. Gouverneur, St.Lawrence.

199 Crystalline limestone (with magnesian nodules). Gouverneur. 200 Primary limestone (brecciated). Gouverneur, St.Lawrence.

201 Crystaldine Limestone (with hornblende). Gouverneur.

202 Crystalidine limestone (with crystals of mica). Antwerp, Jefferson.

203 Limestone (with quartz). Fourth lake, Jefferson. •

204 Limestone (with quartz and felspar). Fourth lake, Jefferson.

205 Serpentine (with quartz). Sterling's mine, Antwerp, Jeffeṛson, 
ROCKS.

Names.

206 Serpentine.

207 Rensselaerite.

208 Rensselaerite.

$209 \& 210$ Silvert talc.

211 Scapolite.

212 BROWN MICA.

213 B

214 FLUOR SPAR.

215 FUSED QUARTZ.

216 QUARTz in chlorite:

217 IRON ORE (scoriaceous).

218 IRON ORE (eOmpaet).

219 IRON ORE (specular).

220 IRON ORE.

221 Limestone with iron ore. Lewisburgh ore bed, Lewis.

222 Limestone (ferruginous). Lewisburgh, Juewis.

223 Crystalitine immestone. Lewisburgh, Lewis.

224 Ferruginous limestone. Near Lewisburgh, Lewis،

225 Impure limestone with iron ore. Lewisburgh, Lewis.

$226 \& 227$ Iron pYRItes. Indian lake, Lewis.

228 Ferruginous limestone. Lewisburgh, Lewis.

229 Specular iron orE. Harrisville, Jefferson.

230 \& 231 Spectlar iron ore (with quartz). Edwards, St.Lawrence.

232 Spectlar iron ore (with quartz crystals). Edwards, St. Lawrence.

233 SPeCULar IRON ORE.

Edwards, St.Lawrence.

234 Sfecular irion ore (with cacozene). Sterling mine, St.Lawrence.

235 SPECUlar IR'N ORE. Harrisville, Jefferson.

236 GNeIss (sienitic).

Near Harrisville, Jefferson.

237 Siliceous limestone. Harrisville, Jefferson.

238 IroN pyrites (in trap). Naturalbridge, Jefferson.

239 Chlorite (the silver ore). Naturalbridge, Jefferson.

240 Serpentine.

241 Sierpentine (brecciated): Antwerp, Jefferson.
Sterling mine, Antwerp, Jefferson.

Naturalbridge, Jefferson.

Edwarảs, St.Lawrence.

Gouverneur, St.Lawrence.

Edwards, St.Lawrence.

Muscalunge lake, Jefferson.

Hammond, St.Lawrence.

Brant lake, Essex?

Lewisburgh, Lewis.

Lewisburgh, Lewis.

Reamer's ore bed, Lewisburgh, Lewis.

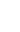

Fowler, St.Lawrence. 


\section{METAMORPHIC ROCKS OF THE MOHAWK GROUP.}

SECOND TABLE CASE.

Names.

1 Quartz Rock.

2 Granular quartz.

3 Compact guartz rock.

4 Granular quartz.

5 Ferruginous quartz.
Localities.

Stanford, Dutchess.

Amenia, Dutchess.

Poughquaick, Dutchess.

Near Peekskill, Westchester.

Williamstown, Massachusetts,

$6 \& 7$ Grandear limestonk. Dover plains, Westchester.

8 Granular limestone. Williamstown, Massachusetts.

$9 \& 10$ Grandtar limestone.Hillsdale, Columbia.

11 - 13 Granular timestoke.Amenia, Dutchess.

14 Granular limestode (clouded). Northeast, Dutehess.

15 Granular limestone (grey). Cortland, Westchester.

16 Granular limestone (variegated). Peekskill, Westchester.

17 Grantular limestone (blue), Peekskill, Westehester.

18 Granular limestone (striped). Near Peekskill; Westchester。

19 Grandlar limestone (compret). Stormville, Dutchess:

20 TALCY LIMESTONE.

Stormville, Dutchess.

21 Talco-slaty limestone. Stormaville, Dutchess.

$22 \& 23$ Talcy limestone. Uniontale; Dutchess.

24 Slaty limestone.

Ancram lear mine, Columbia.

25 Sparry linestone.

Unionvale, Dutchess.

26 Sparky limestone.

Bloomingrove, Orange.

27 Sparry Limestone

Warwick, Orange.

28 - 30 Sparry limestone. Hoosick, Rensselaer.

31 Slaty-talcy limestone. Arthursburgh, Dutchéss.

32 Talco-slaty liméstone, Lebanon springs, Columbia, 
ROCKA.

Names.

33 Staty limestone.

34 Slaty limestone.

35 Statity limestone.

36 Slaty limestone.

37 Mtca slate (with garnets): Dover, Dutchess.

38 Mica and talcy slate.

39 Slate.

40 Talcose slate.

41 Slate (black).

42 Slate (green).

43 Mica state (the alum rocks). Near Ameniaville, Dutchess.

$44 \& 45$ State.

46 Slate (green).

47 Talgose slate.

48 Talcose slate,

49 State.

50 Micaceous slate.

51 Talco-micaceous slate.

52 Chlorite slate.

53 Purple sthate.

$54-57$ Slate.
Localities.

Williamstown, Massãchusetts.

Cortland, Westchester.

Ashford, Massachusetts.

Stanford, Dutchess,

Amenia, Dutchess.

Montrose, Dutchess.

Near Peekskill, Westchester.

Clinton, Dutchess.

Clinton, Dutchess.

Near Canaan centre, Columbia.

Near Washington-hollow, Dutchess.

Canaan-gap, Columbia.

Hillsdale, Columbia.

Hillsdale, Columbia.

Taghkanic falls, Rensselaer.

Richmond, Vermont.

Canaan, Columbia,

Petersburgh, Rensselaer.

Hoosick, Rensselaer.

58 Talcose slate (greenish). Saddle mountain, Massachusetts.

59 Talcose shate.

Tạconic mountain, Rensselaer.

60 Talco-fibrods slate.

Taconic mountain, Rensselaer.

61 Mica slate.

Taconic mountain, Rensselaer.

62 Slaty sanisione.

Villiamstown, Massachuetts.

63 Crystalime quartz (veins in slate). Ancram, Columbia.

64 Crystalline quartz (with copper ore). Washington, Dutchess.

65 Quartz in chlorite (with oxide of manganese). Taconic mountain.

66 Carbonate of iron. Stockbridge, Massachusetts.

67 Limestone (with iron ore). Stockbridge, Massachusetts."

68 TALC and Mica cemented by oxide of iron. Stockbridge, Mass.

69 Hematitic iron ore (stalactitic). Stockbridge, Massachusetts. 
SECOND WALL CASE.

Names.

Localities.

70 Cimestone (with talc). Williamstown, Massachusetts,

71 White LIMESTONE.

New-Ashford, Massachusetts.

72 W HITE LIMESTONE.

Near East-Dover, Dutchess.

73 Cryataline limestone. Singsing, Westchester.

74 Crystalline limestone (dolomitic). Singsing, Westchester.

75 Dolomitic limestone (grey). Singsing, Westchester,

76 Granular limestone (blue). Singsing, Westchester.

$77 \& 78$ Crystalline limestone. Whiteplains, Westchester,

79 Compact limestone. Beekmanville, Dutchess.

$80 \& 81$ Crystalline limestone. Bedford, Westchester.

$92 \& 83$ Crystalline limestone (white). Eastchester, Westchester,

84 Crystalline limestone (bluish). Westfarms, Westchester.

$85 \& 86$ Crystalline limestone (white). Kingsbridge, New-York

$87 \& 88$ GrandLar linestone (white). Verplanck, Westchester.

89 Grantlar limestone (grey). Dover plains, Dutchess.

90 Slaty and clouded limestone. Amity, Orange.

91 Grei lamininated limestone. Adams, Massachusetts.

92 White limestone (with talc). New-Ashford, Massachusetts.

93 White limestone (sawed slab). New-Ashford, Massachusetț,

94 Limestone (with talc). New-Ashford, Massachusetts.

95 Buff-colored Limestone. Williamstown, Massachusetts.

96 Mrlky quartz. Williamstown, Massachusetts.

97 Hornstone in Limestone. Williamstown, Massachusetts.

98 - 100 Limestone with serpentine, Near Putnam courthouse,

101 Talco-serpentine hock. Near Peekskill, Westchester.

102 Serpentine rock.

Phillipstawn, Putnam.

103 Fibrous SERtentine,

Troy, Vermont.

104 Mica slate.

Near Verplanck, Westchester,

105 BLACK SLATE.

Bennington, Vermont.

106 Talco-slate (greenish). Taconic mountain.

107 Slate with QUartz veINs, Williamstown, Massachusetts, 
ROCKs.

Names.

108 SLATE.

109 Talco-slate (greenish), 110 State.

111 Green sLate.

112 Hematitic iron ore. Stockbridge, Massachusetts.

113 Red chalk.

114 Shaț QUaRTZ.
Localities.

Williamstown, Massachusetts, Richmond, Vermont. Taconic mountain. Cortland, Westchester. Williamstown, Massachusetts, 


\title{
III. NEW-YORK SYSTEM,
}

\section{POTSDAM SANDSTONE.}

\author{
TEIRD TABLE OASE.
}

Names. Localities.

1 Potsdam sandstone (red). Dekalb, St.Lawrence.

2 do

3 do

4 do

5 do

6 do

7 do

8 do

9 do

10 do

11 do

12 do

13 do

14 do

15 do

16 do

17 do

18 do

19 do

20 do

$21 \& 22$ do

23 do

24 do

25 do (grey). Moira, Clinton.

(conglomerate). Near Plattsburgh, Clinton,

(red). West-Chazy, Clinton.

(with ripplemarks). Port Kent, Clinton.

(red). West-Chazy, Clinton.

(fragmentary, red). Dekalb, St.Lawrence.

Keeseville, Clinton.

(grey). Klip hill, Johnstown, Montgomery,

Lewisburgh furnace, Lewis.

(conglomerate). Near Lewisburgh furnace. (conglomerate, grey).

(red). Military road west of Plattsburgh.

(metamorphic).

(grey).

(ripplemarks).

Near Lewisburgh furnace, Lewis.

(conglomerate). Near Lewisburgh furnace.

Lewisburgh ore bed.

Lafarge's, near Harrisville.

West of Johnstown, Montgomery.

Klip hill, Johnstown, Montgomery,

Near Lewisburgh furnace, Lewis,

(with lingulæ). Birmingham, Clinton, 
Names.

Localities.

26 Potsdam sandstone. Chazy, Clinton.

27 do

28 do

29 do

30 do

$31 \& 32$ do

$B 3$ do

34 do

35 do

36 do

37 do

38 do

$39 \& 40$ do

41 do

42 do

43 do

44 do

45 do

$46 \& 47$ do

Keeseville; Essex.

(with lingulæ). French creek, St.Lawrence.

(with Lingula antiqua).

(with lingulæ: spotted with manganese).

Near Lewisburgh furnace, Lewis.

Port Kent, Essex.

(containing concretions).

(concretion).

Potsdam:

Saranac.

(colored with vegetable matter). Keeseville, Essex. (containing coneretionary carbonate of lime). (red).

(brecciated),

(fucoidal).

(brecciated), Chazy; Clinton.

(weathered surface). French creek, Jefferson.

(metamorphic).

THIRD WALL CASE:

48 Potsdam sandstone (lowest layer brecciated). Dekalb, St.Lawrence.

$49 \quad$ do

50 do

51 do

$52 \quad \mathrm{do}$

53 do

$54-56$ do

57 do

58 do

$59 \quad$ do

60 do

$61 \& 62$ do

63 do

64 do (massive). Potsdam, St.Lawrence.

(ripplemarks). Keeseville, Essex.

(with casts of mud furrows).

Washington county.

(lowest layer conglomeritic). Lewisburgh, Lewis.

Lewisburgh, Lewis.

Delalb, St.Lawrence.

Washington county.

Chazy, Clinton.

Keeseville, Essex.

Galway, Saratoga.

(with fragments of impure limestone). Chazy. (columnar from artificial heat). Keeseville, Essex. 


\title{
2. CALCIFEROUS SANDSTONE.
}

\author{
FOURTH TABLE OASE:
}

Names.

Localities.

1 - 3 Calciferous sandatone (conglomeritic); Spruce creek, Salisbury, Herkimer.

$4 \div 6 \quad$ do

$7 \& 8$ do

$9 \& 10$ do

11 do

$12 \& 13$ do

$14 \& 15$ do

16 do

$17 \& 18$ do

19 do

20 do

21

do

22

23

24

25

26

27

28

29

30

31

32

33

34

35

do

36

Spruce creek, Herkimer.

(porphyritic). Lead mine, Salisbury, Herkimer.

(erratic). East creek, Herkimer.

(brown). East of Amsterdam, Montgomery.

East of Canajoharie, Montgomery.

(with calcareous spar). East of Canajoharie.

(with calcareous spar). Railroad, Tripe's hill.

Canal west of Littlefalls, Herkimer.

(with globules of anthracite). Littlefalls.

(with anthracite and calcareous spar). Creek at

Spraker's basin, Montgomery.

(anthracite from). Middleville, Herkimer.

West of Littlefalls, Herkimer.

(calcareous, with fossils). Quarry at Canajoharie.' (calcareous, greenish). Canajoharie.

Near Amsterdam, Montgomery.

(with quartz crystals, brown).

(with quartz crystals). Middleville, Herkimer.

(crystals of quartz from). Middleville, Herkimer.

(with calcareous spar). Littlefalls, Herkimer.

(with drops of anthracite). Littlefalls, Herkimer.

(calcareous, with Ophileta). Canajoharie.

(calcareous, with fossils).

(with Lisingula). Canajoharie, Montgomery.

(with Orthoceras primigenium). Opposite Fort-

plain, Montgomery.

(with Fucoides). Canajoharie, Montgomery.

(upper part of mass). Canajoharie, Montgomery. 
Names.

Localities.

37 Calciferous sandstone (shaly, with Palcophycus tubularis). Opposite Fortplain, Montgomery.

38 do (brecciated). Tast-Canada creek, Montgomery.

39 do (casts of mud cracks). Creek S. of Canajoharie.

$40-44$ do (from the altered rocks on the Hudson river.)

45 do (calcareous).

I do not regard it as proved that the last six specimens belong to the Calciferous sandstone proper. J. H.

THIRD WALL CASE.

46 Calciferous sandstone (a red siliceous limestone?). Bellvale, Orange.

47 do

$48 \quad$ do

$49 \quad$ do

50 do

51

52

53

54

55

$56 \& 57$ do

58 do

59 do

60 do

61 do

62 do

63 do

64 do

65 do

66 do

67 do

68 do

$69 \& 70$ do (with drusy quartz). Littlefalls, Herkimer.

Littlefalls, Herkimer.

(with anthracite). Littlefalls, Herkimer.

Glensfalls, Warren.

(with anthracite).

(with trap). Canajoharie, Montgomery.

Canajoharie, Montgomery.

(upper part of the rock). Canajoharie.

Littlefalls, Herkimer.

Canajoharie, Montgomery.

Newport, Herkimer.

(with anthracite). Littlefalls, Herkimer.

(with calcareous spar).

(trap dyke in).

(with anthracite). North of Littlefalls, Herkimer.

(trap in). Wellsborough falls.

(greenstone trap in).

(with pearl spar).

(with Orthoceratite). Fortplain, Montgomery,

(cherty) Galway, Saratoga.

Galway, Saratoga 
Names.

Localities.

$71 \& 72$ Calciferous sandstone. Canajoharie, Montgomery.

$73 \& 74$ do

75 do

76 do

77 do

78 do

79 do

$80 \& 81$ do

82 do

83 do

84 do

85 do

$86 \& 87$ do

88 do

89 do

90 do

91 do

92 do

93 do

94 do

95 do

96 do

97 do

98 do

$99 \& 100$ do

101 do

102 do

103 do

104 do

105 do
Chazy, Clinton.

(with sulphate of iron).

(with lenticular carbonate of lime), Middleville,

(with crystals of quartz and lime).

(hexahedral prism of carbonate of lime from).

(with pearl spar and lenticular carbonate of lime),

(with pearl spar). Littlefalls, Herkimer.

(coarse oolite from). Littlefalls, Herkimer.

Washington, Dutchess.

(Ophileta). Near East-Canada creek.

Galway, Saratoga.

Fortplain, Montgomery.

(chert from). Herkimer, Herkimer.

(drab-colored layer, with spar). Depeauville,

Warwick, Orange.

Amity, Orange.

Littlefalls, Herkimer.

Ogdensburgh, St.Lawrence.

Galway, Saratoga.

Glensfalls, Warren.

(oolitic). Galway, Saratoga.

(oolitic). Chazy, Clinton.

Glensfalls, Warren.

Galway, Saratoga.

(oolitic). Whitehall, Washington,

(encrinal mass in). Chazy, Clinton,

(encrinal layer in). Chazy, Clinton.

Fortplain, Montgomery.

(with Orthis). Cbazy, Clinton, 


\section{BLACK-RIVER LIMESTONE GROUP,}

INCLUDING THE CHAZY, BIRDSEYE AND BLACK-RIVER LIMESTONES.

\section{FIFTH TABLE CASE.}

Names.

Localities.

1 \& 2 Chazy limestone (lower layers). Chazy, Clinton.

3 do (encrinal layers). Chazy, Clinton.

4 do (encrinal mass). Chazy, Clinton.

5 do (encrinal layer with dises and plates). Chazy.

6 do (with fossil shells). Chazy.

7 do (oolitic). Chazy.

8 do (oolitic). Big island, Orange.

9 do (compact). Mount Lookout, Orange.

10 Polished surface of the Encrinal layer. Chazy, Clinton.

$11 \& 12$ Chazy limestone (with Maclurea magna). Chazy.

$13 \& 14$ do (with fragments of fossils). Chazy.

15 do (two specimens, with fragments of shells). Chazy.

16 do (two, with Maclurea and Raphistoma). Chazy.

17 do (with Atrypa and crinoidal columns). Chazy.

18 Birdseye timestone (with Orthoceras and Phylopsis). Great bend of the Black river.

19 do (with fossils). Creek near Spraker's basin.

20 do (with fragments of trilobites). Watertown, Jefferson.

21 do (with obscure fossils). Sugar river near Boonville.

22 do (with Phytopsis cellulosum). Near Watertown.

$23-26$ do (with Phytopsis tubulosum). Fortplain.

27 do (with P.tubulosum). Near Boonville, Lewis.

$28-30$ do Fortplain, Montgomery.

31 do (polished surface, exhibiting transverse sections of $P$. tubulosum). Fortplain.

Near Boonville, Lewis.

(with Phytopsis). Near Boonville, Lewis.

36 do

(with Phytopsis). Near Amsterdam, Fulton. 
Names.

37 BASE of Phytopsis).

38 Birdseye Limestone.

39 Black-RIVER limestone (with Columnaria alveolata). Watertown.

40 do (with C. alveolata). Amsterdam.

41 do (concretionary). North of Amsterdam, Fulton.

42 do (concretionary). Goshen, Orange.

43 do (with Ormoceras tenuifilum). Watertown.

$44-46$ do (with $O$. tєnuifilum). Near Littlefalls, Herkimer.

FOURTH WALL CASE.

46 - 49 Chazy uimestone (with Atrypa, etc.). Chazy, Clinton.

50 do (with Scolithus). Clinton.

51 do (with Maclurea). Chazy.

52 do (with coral and Maclurea). Chazy.

53 do (with Maclurea). Chazy.

54 do (remains of Atrypa, etc.). Chazy.

$55 \& 56$ do (marble). Isle Lamotte.

57 do (with Orthoceratite). Chazy.

58 do (with remains of fossils). Chazy.

$59 \& 60$ do (with Orthoceras). Chazy.

$61 \& 62$ do (with Maclurea). Chazy.

63 do (with remains of Atrypa, etc.). Chazy.

64 do (with traces of Maclurea). Chazy.

65 Bradseye limestone (weathered surface). Mohawl valley.

66 do (with Maclurea). Amsterdam, Fulton.

67 do (remains of fossils). Near Watertown, Jefferson.

68 do (with Phytopsis). Chazy? Clinton.

69 do (with Leptena sericea). Near Middleville.

$70 \quad$ do

Chazy, Clinton.

71 do

72 do

73 do

(with Streptelasma). Dunkirk, Lewis.

(with Phytopsis, etc.). Watertown, Jefferson.

(with coral). Lafargeville, Jefferson. 
Names.

Localities.

74 Hornstone (position uncertain). Goshen, Orange.

75 Birdseye limestone. Chazy, Clinton.

$\begin{array}{lll}76 & \text { do } & \text { (with Phytopsis). Watertown, Jefferson. } \\ 77 & \text { do } & \text { (with Phytopsis). Fortplain, Montgomery. } \\ 78 & \text { do } & \text { (with Phytopsis tubulosum). Watertown. } \\ 79 & \text { do } & \text { (with Phytopsis cellulosum). Fortplain. } \\ 80 & \text { do } & \text { Fortplain. }\end{array}$

81 Limestone (with scratches). Crownpoint, Essex.

82 Limestone (with drift scratches), Pillarpoint, Jefferson.

83 - 85 Limestone (with drift scratches); Amsterdam, Fulton.

The last four specimens apparently belong to the Trenton and Blackriver limestones.

86 \& 87 Black-River limestone (with Columnatia). Chazy, Clinton.

$\begin{array}{lll}88 & \text { do } & \text { (with Columnaria). Dry Moose river, Lewis. } \\ 89 & \text { do } & \text { (with Columnaria). Watertown, Jefferson. } \\ 90-93 & \text { do } & \text { (with Orthoctras). Watertown. } \\ 94 & \text { do } & \text { (iridescent). } \\ 95 & \text { do } & \text { (with concretions). St.Johnsville, Montgomery. } \\ 96 & \text { do } & \text { Tripeshill, Montgomery. } \\ 97 & \text { do } & \text { (with Orthoceras). Watertown, Jefferson. } \\ 98 & \text { do } & \text { (with Columnaria). Watertown. } \\ 99 & \text { do } & \text { St.Johnsville, Montgomery. } \\ 100 & \text { do } & \text { (concretionary). St.Johnsville, Montgomery. } \\ 101 & \text { do } & \text { (concretionary). } \\ 102 & \text { do } & \text { (concretionary). Littlefalls, Herkimer. } \\ 103 & \text { do } & \text { (concretionary). Amsterdam, Fulton. } \\ 104 & \text { do } & \text { (concretionary). Littlefalls, Herkimer. } \\ 105 & \text { do } & \text { (striated from movement of strata). } \\ 106 & \text { do } & \text { (with Columnaria). }\end{array}$




\section{TRENTON LIMESTONE.}

SIXTH TABLE CASE.

Names. Localities.

1 - 3 Trenton limestone (with Chatetes). Newport, Herkimer.

4 Large hemispherical form of Chatetes lycoperdon. Newport.

5 The same (weathered specimen): Middleville, Herkimer.

6 Trenton limestone (with Chatetes). Middleville:

7 \& 8 Chatetes (hemispherical form).

9 Trenton limestone (with branching forms of Chatetes lycoperdon). Sugar river, Boonville, Lewis.

10

11 do

12 do

13 do

14 do

15 do

16 do

17 do

18 do

19 do

$20 \& 21$ do

22

do

23 do

$24 \& 25$ do

26

do

27

28

29

30

do

o

o

o

0

(a)

o

.


Names.

Localities.

31 - 33 Trenton limgstone (with Leptana, etc.). Middleville.

34 do

$35 \& 36$ do

$37 \& 38$ do

39 do

40 do

41 do

42 do

43 do

44 do

45 do

46 do

$47 \quad$ do

48 do

49 do

50 do

$51-53$ do

54 do

55

do (with $L_{\mathrm{r}}$ sericea). Fortplain, Montgomery.

(with L alternata). Near Middleville, Herkimer.

(with Orthis pectinella). Middleville.

(with $O$. pectinella and fragments). Middleville.

(with O. testudinaria). North of Littlefalls.

(with $O$. testudinaria), Watertown, Jefferson.

(with Delthyris lynx).

(with Atrypa extans and impressions of Orthis). Near Boonville.

(with Atrypa extans). Watertown, Jefferson.

(with Pleurotomaria, etc.). Watertown.

(with Pleurotomaria and Subulites). Watertown.

(with Endoceras proteiforme). Middleville.

(with fragment of an Orthoceras). Watertown.

(with fragment of Orthoceras). Middleville.

(with fragment of Orthoceras). Fortplain.

(with fragments of Orthoceras). Middleville.

(with fragments of Endoceras proteiforme). Middleville.

(with fragments of $O$. juncerum). Watertown.

56 Endoceras of the Trenton limestone.

57 TRenton LIMEstone (with fragment of Orthoceras?),

FIFTH WALL CASE,

58 Trenton limestone (with Isotelus). Creek at Spraker's basin, Fulton.

59 Fragment of Orthoceras.

60 Trenton limestone (with Leptcena, ete.).

61 Fragments of Orthoceras from the Trenton limestone.

62 Trenton Limestone (with fossils).

63 do (with Isotelus). Glensfalls, Warren.

64 do (with Isotelus). Middleville, Herkimer,

$65-67$ do Glensfalls, Warren, 
Names. Localities.

68 Trenton limestone. Baker's falls, Washington.

69 do (with Trinucleus). Locality unknown.

70 do (with fossils). Locality unknown.

71 do

72 do (with Chetetes lycoperdon). Jacksopburgh, Herkimer.

72 do

(with Trinucleus and other fossils). Near Boonville?

73 do

(with Chatetes lycoperdon).

74 do

(with Calymene senaria). Below Middleville,

75 do

(with Calymene senaria).

$76 \& 77$ do

(with Chatetes).

$78-81$ Hemispherical Chetetes from the Trenton limestone.

82 Trenton limestone (with Orthoceras).

83 Orthoceras.

84 Trenton limestone (with Orthoceras). Dry Moose river, Lewis.

85

86

87

38

89

90

91

92

93

94

95 ḋo

do do do do

do

do

do

do

do

do

do

do

do

do

do

102

do

(with Orthoceras). Middleville, Herkimer. (with Orthoceras). Watertown, Jefferson. (with Subulites). Wattertown, Jefferson.

Sugar river near Boonville, Oneida. (with Pleurotomaria, Murchisonia, etc.). Trenton, (with remains of Trinucleus, etc.). Tribeshill. (with Orthoceras): (with fragments of fossils). (with Orthis pectinella). (with Leptana alternata, young shell). (with Atrypa extans). (with Pleurotomaria lenticularis). (with Leptana sericea). (with L. sericea and athers). (with Orthis testudinaria). West-Canada creek. (with Leptana alternata). (with Orthis and Leptana). Near Boonville. do do 
Names.

Associated fossils and localities.

103 Trenton limestone (with Orthis and Leptena). Dry Moose river, Lewis.

104 do

105

106

107

108

109

110

111

112

113

114

115

116

117

118

119

120

121

122

123

124

125

126

127

128

129

130

131

132

133

134 do

do

do

do

do

do

do

do

do

do

do

do

do

do

do

do

do

do

do

do

do

do

do

do

do

do

do

do

do

do

(with fragments of fossils).

(with Chatetes lycoperdon).

(with remains of fossils).

(hemispherical form of Chatetes from).

(with Conularia). Sugar river near Boonville.

(with remains of Leptena).

(with L. sericea). Sugar river, Boonville.

(with Orthis pectinella). Middleville.

(with Leptana sericea). Dry Moose river.

(with graptolites). Trenton falls.

(with spar). Sugar river, Boonville.

(with Orthis testudiraria). Fortplain.

(with O. testudinaria).

(with encrinal and other remains).

(with obscure Leptana alternata). Martinsburgh. (polished specimen). Boonville, Sugar river.

(with encrinal stems).

(with caudal shield of Illoenus crassicauda).

(with encrinal fragments). Near Trenton village.

(with fragment of Isotelus gigas). Near Boonville.

(with fragments of Calymene). Middleville.

(with encrinal and other remains).

(with fragments of Orthoceras, etc.). Watertown. (with Orthis, etc.). Watertown.

(with crinoidal fragments and concretions). Creek at Spraker's basin.

(with casts of Isotelus gigas). Plattsburgh.

(with Orthis pectinella).

(with obscure fossils). Dry Moose river.

(with Orthis). Falls of Sugar river, Boonville.

(with fragments of Pleurotomaria). Watertown.

(with fragments of fossils, crystalline). 
Names.

Associated fossils and localities.

135 Trenton limestone (with Orthoceras). Middleville.

136 do (with spar). Middleville.

137 do (with fragments of fossils). Middieville.

138 do (with calc spar). Falls at Lowville.

139 do (with base of Chatetes and shells).

140 do (with fragments of fossils, crystalline). Hawkins's mill, Boonville road.

141 do (with Leptana and fragments of other fossils). Lower falls of Lansing's kill.

$142-145$ do (crinoidal fragments). Quarry near Trenton village.

$146 \& 147$ do (mass of fossil fragments, crystalline). Near Treñton village.

148 do (with fossils and coneretions). $\Lambda$ msterdam.

149 do (with spar). Martinsburgh

150 do (with lead ore). Martinsburgh.

151 do (with Chatetes, branched variety).

$152 T_{\text {RAP }}$ DYKe (in Trenton limestone). East-Canada creçk.

153 \& 154 Porphyry (in Trenton limestone). Essex.

\section{UTICA SLATE.}

SEVENTH TABLE CASE.

1 Utica slate (with graptolites). East-Canada creek.

2

$\begin{array}{ll}3 & \text { do } \\ 4 & \text { do } \\ 5 & \text { do } \\ 6 & \text { do } \\ 7 & \text { do } \\ 8 & \text { do }\end{array}$

(with fragments of Calymene beckii). Mohawk valley.

(with Leptana sericea). Creek at Johnstown. (with Orthis testudinaria). Creek at Johnstown. (with heads of Calymene beckii). Oxtungo creek, south of Fortplain.

(with Calymene beckii). Oxtungo creek. (with Graptolithus lovis). Turin, Lewis. (with graptolites). Johnstown creek. 
Names.

Associated fossils and localities.

$9 \& 10$ UTICA SLATE (with graptolites). Oxtungo creek.

11 do

12 do

13 do

14 do

15 do

16 do

17 do

18 do

19 do

20 do

21 do

$22 \& 23$ do

24 do

25 do

26 do

27 do

28 do

29 do

30 do

31 dó

32 do

$33 \& 34$ do

35

do

36

37

38

39 (with graptolites). Creek north of Herkimer. (with Orthoceras). Turin.

(with Orthoceras). East-Canada creek. (with graptolites and Pleurotomaria). Coldspring. (with Calymene). Near Amsterdam.

(with fragments of graptolites). (with head of Calymene beckii). Turin. (with Calymene beckii). Whetstone creek, Lewis. (with fragments of Calymene). Whetstone creek. (with fragments of C. beckii). Pulaski, Oswego. (with fragment of Orthoceras). East-Canada creek. (with fragments of Orthoceras). Whetstone creek. Loraine, Jefferson.

(with fragments of Calymene, Graptolithus, etc.). Pulaski and Loraine.

(with fragments of fossils). Lsoraine.

(with fossils in an erratic mass). Near Littlefalls. (with Orthoceras). Whetstone creek, Lewis. (compact, with iron pyrites). East-Canada creek. (compact, with a concretion). Near Middleville.

Canajoharie.

(with graptolites).

(with graptolites and crystals of gypsum). Baker's falls, Washington.

(glazed). Chatham, Columbian

(glazed). Near Poughkeepsie.

Reed's spring, Washington.

(with graptolites). Kinderhook creek, Columbia. (metamorphic). Near Hudson. 


\title{
6. HUDSON-RIVER GROUP.
}

\author{
EIGHTH TABLE CASE.
}

Names.

Associated fossils and localitieg.

1 Hudson-River slate (with Cheetetes, branched form). Loraine, Jeffetson.

2 do (with encrinal stems and obscure fossils). Loraine:

3 do (with encrinal fragments). Lee centre.

4 do (with obscure remains). Loraine.

5 Hudson-River shale (with fragments of fossils). Pulaski, Oswego,

6 do (calcareous, with encrinal stems and fragments). Pulaski:

$7 \& 8$ do (calcareous, with Leptana alternata). Pulaski.

9 Hudson-river calcareous sandstone (with Modiolopsis). Pulaskis

10 do (with Ambonychia and Orthis). Turin, Lewis.

11 do (with fragments of fossils). Walden.

12 do (with Mrodiolopsis). Loraine.

13 HUDSON-RIVER SANDSTONE, calcareous and shaly, deeomposing (with shells). Pulaski.

14 do (with fragments of Leptcena, etc). Pulaski.

15 do (with obseure impressions of Tentaculites and Leptrena). Whetstone creek, Lewis.

16 do (shaly and caleareous, decomposing, with Ambbnyctia and numerous other fossils). Pulaski.

17 Shaly \& calcareods sandstone (with Modiolopsis, etc.). Pulaski.

18 Hudson-river calcareous sandstone (with fossil fragments). Pulaski.

19 do (decomposing, with impressions of Orthis testudi naria). Pulasks.

20 do (with encrinal joints, Orthis, etc.)s Loraino.

21 do (with encrinal joints, Ambonychia, etc.). Loraine.

22 do (with Ambonychia radiata). Loraine.

23 IIUdson-River calCARtous sHaLE (with encrinal columns and other fossils). Loraine. 
Names.

Associated fossils and localities.

24 HUDSON-RIVER SANDSTONE (with obscure remains of Chatetes, Orthis, etc.). Loraine.

25 do

(with Ambonychia and Orthonota). Talcott's quarry, near Rome, Oneida.

26 do (with Ambonychia). Talcott's quarry.

27 do (with Modiolopsis). Talcott's quarry.

28\&29 HudSON-RIVER CAECAREOUS SANDSTONE (with Leptrera alternata). Pulaški, Oswego.

30 HUdison-RIVER CALCAREOUS SANDSTONE (with obscure fossil impressions). Falls of Salmon river.

31 HuDson-RIver calcakeous shade (with obscure.fossils). Northwest corner of Columbia county.

32 Hudson-river calcareous shaie (with crinoidal plates and shells). Pulaski, Oswego.

33 Hudson-RIVER DECOMPOSING SHALX SANDSTTONE (with Cyrtolites ornatus). Loraine, Jefferson.

34 Hudson-ritver calcareous sandstone (with Bellerophon bilobatus). Loraine.

35 Hubson-RIVER sandstone (with Orthoceras). Oneida،

$36 \& 37$ Hudson-river shaly sandstone, decomposing (with Leptcona, etc.). Pulaski.

38 Hulsonariver calcareous sandstone (with fossil fragments). Pulaski.

39 HUdSON-RIVER SANDSTONE (with Leptana alternata, etc.), Loraine.

40 Hudson-River calcareous sandstone (with Chatetes \& fragments). Talcott's quarry.

41 Hubson-biver calcareous sandstone (with Ambonychia). Oneida.

42 Hudson-river CalCareous SANDSTONe (with obscure impressions).

SIXTH WALL CASE.

43 Shady sandstone. Snake hill, Saratoga。

44 Green shale.

Snake hill.

45 Gray sandstone.

Redhook, Dutchess.

46 Green shale.

Snake hill, Saratoga.

$47 \& 48$ Black sLate (with graptolites). Columbia county.

49 Shale.

Banks of North river, Dutchess. 
Names. Locálities.

50 Green slate.

Cornwall, Orange.

51 Green (calcareous) shale (with fossils, Nucula, etc.). Whitt's quarry.

52 Siliceous black slate. Clermont, Columbia.

53 Green siliceous slate. Montrose, Dutehess.

54 Sandstone, slaty (slaty graywacke). Redhook.

55 Shaly sandstone.

Stockport, Columbia.

56 Black slate.

Hydepark, Dutchess.

57 SAndstone (compact and partially altered). Bloomingrove, Orange.

58 Siliceous slate.

Cornwall, Orange.

59 Sandstone (laminated and micaceous). Minisink, Orange.

60 SLATE, black (thinly laminated). Clermont, Columbia.

61 Siate, black (thinly laminated). Sugarloaf, Orange.

62 Sandstone (laminated and micaceous). Walden, Orange.

63 Slaty SANDstone (with mica and grains of anthracite). Walden.

64. Fuinty slate (black). Hudson, Columbia.

65 Flinty slate (greenish). Hudson.

66 Laminated sandstone. Schoharie.

67 Gray sandstone. Banks of Hudson river, Dutchess.

68 Black slate. Hillsdale, Columbia.

69 Gray sandstone (siliceous and partially altered). Ghent, Columbia.

70 Gray sandstone (conglomeritic). Banks of Hudson river, Dutchess.

71 Gray sandstone (slightly conglomeritic and laminated). Hydepark landing, Dutchess.

72 Siliceous slate (with copper and iron pyrites). Near Lower Redhook.

73 Flinty slate.

Near Hudson, Columbia.

74 BRown Jasperi y slate. Near Kinderhook creek, Chatham, Columbia.

75 Glazed slate.

Near Rider's mills, Chatham.

76 GLazed sLate.

South of Hudson, Columbia.

77 Glazed slate, greenish (shining argillite). Rider's mills, Chatham.

78 Siliceous and Glazed slate (with limestone). Chatham.

79 QUartz CRYSTALS, in cavities of quartz. Near Lansingburgh.

80 RED SLATE.

South of Hampton, Washington.

81 Siliceous slate.

Lower Redhook landing, Dutchess. 
ROCKS.

Names.

Localities.

82 State.

Union corner, Hydepark, Dutchess.

83 'Siliceous slate (greenịsh). Banks of Hudson river, Dutchess.

84 State with iron pyrites (thinly laminated). Hillsdale, Columbia.

85 BLACK slate (thinly laminated). Hillsdale.

86 Roofing SLate. Gillet's quarry, Lebanon, Columbia.

87 State (micaceous and laminated). Redhook, Dutchess.

88 \& 89 Talcose slate (contorted). Whiting's pond, Canaan, Columbia.

90 Green slate, siliceous. Near Albany.

91 Red Jaspery slate. Lower Redhook landing, Dutchess.

92 Brown Jaspery slate. Bloomingrove, Orange.

93 Slate with cast of mud-furrows. Frankfort creek, Herkimer.

94 Siriceous slate with irregular surface (mud markings). Oneida.

95, 96, 97 Black slate (with satin spar). St.Albans, Vermont.

98 Silickous slate (altered). Willsborough, Essex.

99 Sandstone (gray, siliceous). Oneida creek.

100 Red slate.

Washington?

101 Greenish sandstone (argillaceous). Falls of Salmon river, Oswego.

102 GreEnish sandstone (with surface markings). Falls of Salmon river.

103 Ferruginous sandstone (with quartz veins).

I04 Green slate. Ridge mill near Rome, Oneida.

105 Shaly sandstone (with quartz erystals).

$106 \& 107$ Green shaly sandstone (with calcareous spar).

108 \& 109 Green shale. Barney's, north of Rome, Oneida.

110 Compact dark slate. Summit pond, Argyle, Washington.

111 Dark green state. Wynn creek.

112 Black thinly laminated slate. Vanhorn's mill near Rome.

113 Compact siliceous slate. Near Rome, Oneida.

114 Arenaceous limestone (with Ambonychia and Cyrtolites). Lewis.

115 Orthoceratite. Lewis county.

116 Shaly sandstone (with Ambonychia radiata). Loraine, Jefferson.

117, 118, 119 Sandy Shale (with crinoidal joints, Orthis, ete.). Lioraine.

120 Shaly limestone (with Orthis, Orthoceras, etc.). Iuoraine. 
Names.

Associated fossils and localities.

121 Gray sandstone (with fossils). West of Constableville, Lewis.

122 SiLicEors Mass in black shale. Lansingburgh, Rensselaer.

123 Siaty argildaceods limestone. Greenwich, Washington.

124 Sandstone (with small branching Chatetes lycoperdon). Oswego.

125 Calcareous sandstone (with Ambonychia radiata).

126 Gr.ax sandstone (with Ambonychiut, etc.). Near Rome, Oneida.

127 BLACK SLATE (thinly laminated). Vanhorn's mill near Rome.

128 Grayish Green shale. Snake hill, Saratoga.

129 Calcareous shale (with columns of Heterocrimus). Oneida.

130 Shaly sandstone (with Orthoceras). Loraine, Jefferson.

131 Shaly sandstone (with Modiolopsis ovata). Loraine.

132 Calcareods sanditone (with Ambojuychia radiata). Pulaski.

133 Shaly sandstone (with Leptcena sericea). Near Copenhagen, Lewis.

134 Compact sanstone. Talcott's quarry near Rome.

135 GreEN shale. Saugerties, Ulster.

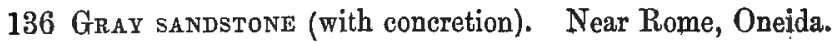

137 Linesmone with fossils (intercalated with the Hudson-river shales), Near Barnegate, Dutchess.

138 Sandstone (with Orthoceratites, Cyrtolites, etc.). Turin, Lewis.

139 Shaly sandstone (ferruginous and decomposing). Lorạime?

140 Gray sandstone (with fragments of fossils). Near Constableville.

141 GreEn siliceous slate (whetstone slate). Columbia county.

142 Compact shaly Sandstone (with quartz crystals). Near Albany,

143 Glazed slate.

Near Hudson.

144 Thinbedded sandstone (with fossils). Near Russia, Herkimer.

145 Slaty calcareous rock (with Orthis testudinaria). Lewis,

146 Siliceous limestone (interstratified with slate). Columbia;

147 Shapy sandstope. Rogers's creek, Oneida. 


\title{
7. GREY SANDSTONE.
}

\author{
\$EVENTH TABLE CASE.
}

Names.

Localities, etc.

1 Roof slate. Rowley's quarry, New-Lebanon, Columbia county.

2 Slate. One mile west of Hillsdale, Columbia.

3 Glazed slate. One mile south of Hudson, at the supposed coal mine.

4 Red slate. Near Kinderhook, Chatham, Columbia.

5 Red slate. One mile southwest of Washington-hollow, Dutchess.

6 Millstone Grit. Steel's creek.

$7,8,9$ Shaly sandstone (with fragments of fossils).

10 Shaly sandstone (with impressions of Modiolopsis modiolaris).

11 Shaly sandstone.

12 Concretionary gandstone.

13 Gray shaly sandstone. Two and a half miles north of Rome.

14, 15, 16 Gray sandstone. Woodruff's, south of Rome.

17 Greensah giazed state.

18, 19 Gray Sandstone. Salmon creek, village of Florence.

20 Gray sandistone. Falls of Stony brook, Redfield.

21 Grax sandstone. Village of Florence.

22 Gray sandstone (with water-marked surface). Falls of Salmon creek.

23 Gray sandstone. West of Oswego village.

24 Gray sandstone. Woodruff's quarry, south of Rome.

25 Gray sandstone. Below the dam on Mad river, Camden.

26 Gray sandstone. Dam at Mad rivor, near Camden.

27 Gray sandstone. Upper falls of Mad river, Redfield.

28 Gray sandstone. Road to Loraine from Redfield.

29 Gray sandstone. West of Ghent meeting-house, Columbia.

30 Gray sandstone. Road south of Rome, Oneida.

31 Gray sandatone. Tippler's quarry:

32, 33, 34 Gray șandstone (with Strophomena alternata).

85 Gra Y glLICEOUS SANDSTONE (with shaly nodules, ạd containing galepa). 
Names. Localities, etc.

36 Contorted linestone. Near Stanfordville, Dutchess.

37 Milistone grit. Bloggs's clove, Bloomingrove, Orange.

38 Compact sandstone. Twoponds, Monroe, Orange.

39 Millstone grit (boulder). Hamptonburgh, Orange.

40 Millstone GrIt. Deerpark, foot of Shawanguuk mountain range.

41 Graywacise. Two miles west of Woodbury, Monroe, Orange.

42 Millstone grit. Deerpark, Orange.

43 Conglomerate. Canterbury, Orange.

44 Finegrained grit rock. Shawangunk mountain, Rochester, Ulster,

45 Grit Rock. Walls of the Shawangunk lead vein, Sullivan.

46 Millstone grit. Deerpark, Orange.

47 Breccia. Two miles northeast of Craigville, Orange.

48 Yellowish sandstone. Stockport landing, Columbia.

49 Compact shaly sandstone. Near Hydepark, Dutchess.

50 A METAMORPHIO SLATY AND CRYSTALTiNE ROCK. —?

51 CoMract metamorphic gRIt Rock. - ?

52 Brownish siliceous limestone (metamorphic). ? ?

53 Compact shaly sandstone.

54 Shaly sandstone (with thin layers of shale). - ?

55 Siliceous Limestione.

56 Limestone. Bloggs's elove, Bloomingrove, Orange.

57 Linestone. Warwick, Orange. SEVENTH WALI CAST.

58 Grey sandstone. Three miles north of Salem, Washington.

59 Black slate. Hoosick, Rensselaer.

60 Linestone. West of Granville, Washington;

61, 62 Limestone. Snakehill, Saratoga.

63 ReD shale. Eaton, Washington.

64, 65 Shale. Sugarloaf, Orange.

66 Compact sandstone. Bellvale, Warwick, Orange.

67 Red slate. Bloomingrove, Orange.

68 Millstone Grit (compact). Canterbury, Orange.

69 Red shaly sandstone. Canterbury.

70 Red sandstone. Pine hill, Cornwal, Orange.

71. Red millstone grit, Carpenter's point, Deerpark, Orange,

72 Red shale. Cambridge, Washington. 
EIGHTH TABLE CASE.

Names.

Localities, etc.

1, 2, 3 Onima conglomerate. Steel's creek, Herkimer.

4 Oneida conglomerate. Cleveland, Oneida lake.

5,6 do

7 do

8 do

9 do

10,11 do

12 do

13 do
Mason's quarry, southwest of Utica.

Mansfield, south of Hampton.

South of New-Hartford, Oneida.

Mason's quarry.

Near Starch-factory creek, Oneida.

Steel's creek, Herkimer.

Mansfield, south of Hampton.

14 Milistone Grit. Hill near Starch-factory creek, Oneida.

\section{MEDINA SANDSTONE.}

EIGHTH TABLE CASE.

15, 16 Medina sandstone (gray beds). Lewiston, Niagara county.

17 Medina sandstone (with ripplemarks). Juewiston, high bank of river.

18 do (gray beds). Lewiston.

19 do (with undulating lines of deposition). Martville, Cayuga.

20 do Wayne county.

$21-23$ do

Near Martville, Cayuga.

24 do

Hulme's quarry, Sterling, Cayuga.

25 do

(with Dictuolites beckii). Metina, Orleans.

26 do

(upper mass). Hulme's quarry, Sterling.

27,28 do

(upper mass). Cental's mill, Wayne.

29,30 do

Hulme's quarry, Sterling.

31,32 do

Near Martville, Cayuga.

33 do

(upper gray mass). Medina.

34 do

(upper gray mass). Lewiston.

35 do

(upper gray mass). Rochester. 
NINTH TABLE CASE.

Names. Localities, etc.

1 - 5 Medina sandstone. Sterling centre, Cayuga county.

6 Medina sandstone. Park's milldam, Amboy, Oswego.

\begin{tabular}{|c|c|c|}
\hline 7 & do & (with concretionary markings). Oswego village \\
\hline 8 & do & (with mud cracks). Oswego village. \\
\hline 9,10 & do & (with Pleurotomaria pervetusta). Medina. \\
\hline & do & (with $P$. pervelusta \& Lingula cuneata). Medina. \\
\hline-14 & do & (with $P$ : pervetusta). Medina. \\
\hline & do & East side of Irondequoit bay. \\
\hline & do & (with Palcophycus tortuosus). Brighton, Monroe. \\
\hline-22 & do & (with Lingula cuneata), Medina. \\
\hline & do & (with Palaophycus). Medina. \\
\hline-28 & do & $\begin{array}{l}\text { (with Modiolopsis primigenius). Medina. } \\
\text { (with fucoids). - ? }\end{array}$ \\
\hline & do & $\begin{array}{l}\text { (with Arthrophycus harlani, loose). Near Cleve- } \\
\text { land, Oneida lake. }\end{array}$ \\
\hline & do & (with $A$. harlani). Medina. \\
\hline & do & Near Sterling, Cayuga. \\
\hline & do & (with Arthrophycus harlani). Medina: \\
\hline & do & (with $A$. harlani \& Fucoides? auriformis). Medina. \\
\hline & do & (with Dictuolites beckii). Medina. \\
\hline
\end{tabular}

SEVENTH WALT CASE.

35 Medina sandstone (with Lingula cuneata, showing current markings)。 Lockport, Niagara county.

36 do (geode). Lockport.

37 do (upper gray layers). Medina, Orleans.

38 do (variegated). Rochester, Monroe.

39 do Lewiston, Niagara.

40 do (upper gray layer). Lewiston.

41 do (conglomerate). Wolcott, Wayne.

42 do (with water-markings). Lewiston.

43 do (showing diagonal lines of lamination). Rochester. 
Names.

Localities, etc.

44 Medina sandstone. Martville, Cayuga.

45 do

46 do

47 do

48 do

49 do

50 do

51

do

$52 \& 53$ do

54

55

56

57

58

59

60

$61 \& 62$ do

$63 \& 64$ do

65

do

66

67

68

$69-71$ do

72

73

74

75

76

77

78

$79 \& 80$ do (inclosing nodules of shale). Rochester.

(with Arthrophycus harlani). Wayne.

(variegated and shaly). Medina.

(shaly). Lewiston.

Redfield, Oneida.

(upper shaly layers). Medina.

(variegated). Lewiston.

(upper greenish layers). Lewiston.

(upper gray layers, with Cytherind cylindrica). Medina.

(variegated). Near Martville, Cayuga.

(variegated). Lewiston, Niagara.

Rochester, Monroe.

(very shaly). Irondequoit bay, Monroe.

Medina, Orleans.

(with Lingula cuneata and Cytherina cylindrica). Medina.

(with nodules of shale enclosed). Medina.

(with Lingula cuneata). Lockport and Medina.

(with L. cuneata and Pleurotomaria pervetusta).

Medina.

(with L. cuneata). Medina.

Clarendon, Orleans.

(with tortuous lamination). Rochester.

(shaly variety). Lewiston.

(shaly). Rochester, Monroe.

(shaly). Martville, Cayuga.

(with C. cylindrica and L. cuneata). Medina.

(with concretionary surface). Oswego.

(with Arthrophycus harlani). Medina.

(with A. harlani). Adams's basin, Monroe.

(with Fucoides? heterophyllus). Adams's basin.

(with F.? heterophyllus). Medina. 
Námes,

Localities, etc.

81 Medina sandstone (with $F$ :? heterophyllus). Rochester;

$82 \& 83$ do (with fucoidal markings). Medina.

84 do (with fucbidal markings). Martville.

85 do (variegated). Philipsburgh, Oswego.

86 do (with fucoidal markings). Lewiston.

87 do (with Arthrophycus harlani, and conglomerate). Oswego county.

88 do

(with A. harlani). Medina, Orleans.

$89 \quad$ do

90 do

(with ripplemarks)。 Medina.

$91 \& 92$ do

93

do (with $A$.harlani). Sodus, Wayne. (upper gray layers). Medina. Medina.

\section{CLINTON GROUP.}

TENTH TABLE CASE.

1 Conglomerate (with shaly surface). Blackstone's quarry, NewHartford, Oneida county.

2 Shaly sandstone (with Buthotrephis palmata). Blackstone's quarry.

3 Shaly sandstone (with Rusophycus bilobatus). Gaylord and Norton's quarry, New-Hartford.

4 Shaly sandstone (with Buthotrephis gracilis). Blackstone's quarry.

5 Sandstone (with Beyrichia). New-Hartford.

6 Conglomerate. Stebbins's creek, near Clinton village, Oneida.

7 Conglomerate (with iridescent surface). Blackstone's quarry.

$8,9,10$ Oolitic iron ore. Bennett's ore bed, west of Clinton village.

11 Oolitic inoN oRE. Wadsworth's quarry, New-Hartford.

12 do (with crinoidal joints). Eames's quarry, Verona.

13 do (with crinoidal joints). Parsons's quarry, Verona.

14 do Hammond's ore bed, Westmoreland, Oneida.

15 do Parsons's quarry.

16 do (with Leptana sericea). Bennett's ore bed. 
Names.

Associated fossils and localities.

17 Shaly sandstome (with $L$. sericea). South of Verona, Oneida. 18, 19 Limestone (with Pentamerus oblongus). Rochester, Monroe. 20 Siliceous limestone (with $P$. oblongus). Wolcott, Wayne.

21 Limestone (with Atrypa hemispherica). Below Martville, Cayuga.

22 Limestone (with fragments of $P$. oblongus). Sodus, Wayne.

23 Oolitic IRON ORE. Rochester.

24 Oolitic IRON ORE (2 specimens). Stebbins's creel, Oneida.

25 Limestone (with Leptana rugosa). Donnelly's quarry, Madison.

26 Oolitic iron ore. Wolcott, Wayne.

27 do (with fragments of shells). Donnelly's quarry.

28 do (with Pentamerus oblongus). Donnelly's quarry.

$29 \& 30$ do (with Spirifer radiatus). Donnelly's quarry.

31 Fgproginous limestone (with $P$.oblongus). Donnelly's quarry.

32, 33 Shaly sandstone (with Rusophycus pudicus). Gaylord's quarry.

34 Atrypa congesta. Reynale's basin, Niagara.

35 Atrypa plicatella. Reynale's basin.

36 Shale (with Graptolithus clintonensis). Sodus.

37 Limestone (with Atrypa hemispherica). Rochester.

38 Limestone (with $A$. reticularis and $P$.oblongus). Donnelly's quarry.

39 GRAX SANDSTONE (with fragments of Homalonotus delphinocephalus). Steel's creek, Herkimer.

40 Gray sandstone (upper mass of group). Steel's creek.

41 Shale (with Atrypa congesta). Reynale's basin, Niagara.

42 Atrypa plicatella. Reynale's basin.

43 Chatetes lycoperdon. Reynale's basin.

44 Upper gray sandstone (with Modiolopsis ovata). Remington's quarry:

45 Limestone (with Atrypa congesta). Medina.

46 Caninia bilateralis (2 specimens). Reynale's basin.

47 Atrypa congesta.

48 Fibrous sulphate of strontian. Stark, Herkimer.

49 Fibrous sulphate of strontian (with carbonate of lime, etc.). Stark.

50 Crystallized sulphate of strontian. Stark.

51 Gypsum. Stark. 
EIGHTH WALL CASE.

Names.

Associated fossils and localities.

52 Shaly Sandstone (with fucoidal markings). Blackstone's quarry,

53 SHALE (with obscure fossil markings). Blackstone's quarry.

54 Green shale. Martville, Cayuga.

55 Ferruginous sandstone (with Beyrichia). New-Hartford.

56 Shaly sandstone (with Rusophycus subangulatus). Blackstone's,

57 Shaly sandstone (with fucoidal markings). Blackstone's quarry.

58 SHALE (with Buthotrephis gracizis). Near Clinton village.

59 Sandstone (with $B$. palmata). Fox-hollow, Herkimer.

60 Shaly sandstone (with organic markings). Blackstone's quarry,

61 Shaly sandstone (with Rusophycus bilopatus). Gaylord's quarry.

62, 63 ShaLe (with fragments of B. ramosa). Blackstone's quarry.

64 Shaly sandstone (with fucoidal fragments). Near Verona.

65 Conglomerate (with iron pyrites). Blackstone's quarry.

66 do (with iridescent surface). Blackstone's quarry.

67 do (with shaly surface). Stebbins's creek.

68 do (with shaly surface). Rogers's creels.

69 do Blackstone's quarry.

70 Shaly SANdstone (with fucoidal markings). Near Clinton village.

71 Shalt sanpstone (with fucoidal markings). North of Martville.

72 Shale. A quarry north of Martville.

73 Shaly sandstone ( 2 specimens, with fucoidal remains). Clinton,

74 Hornstone (composed of fragments of shells). Rochester.

75 Sandstone (with Beyrichia). New-Hartford.

76 Grey sandstone (with Lingula oblonga). Quarry north of Martville.

77 Ferruginous sandstone (with organic remains). New-Hartford.

78 Ferruginous sandstone. Stark, Herkimer.

79 Ferruginous sandstone. Steel's creek, Herkimer.

80 Shaly sandstone. Vanhornsville, Herkimer.

81 Shaly limestone. Bushnell's, Oneida lake.

82 do Qparry near Verona.

83 do (with Fenestella prisca?): Near Verona. 
Names.

Associated fossils, and localities.

84 Shaly limestone (with Fenestella prisca?). Martville, Cayuga.

S5 Shaly limestone. Martville.

86 Green shale. Martville.

87 Green shale. Swift's creek.

88 Limestone. Near Verona, Oneida.

89 Shaly linestone. Near Verona.

90 Oolitic Iron ore (with Beyrichia). Vanhornsville, Herkimer.

91 do

$92 \& 93$ do

94 do (with concretions and crinoidal joints). Pearson's ore bed, near Verona.

95 Red sandstone. Steel's creck, Herkimor.

96 Red conglomerate. Steel's creek.

97 OOLITIC IRON ORE (with limestone). Wolcott, Wayne.

98 Limestone (with Pentamerus oblongus). Sodus, Wayne.

99, 100 Limestone (with P.oblongus). Rochester, Monros.

101 Shale (with Leptcena rugosa). Donnelly's quarry, Madisun.

102 OOLITIC IRON One (with calcareous spar). Wolcott.

108 Shaly dimestone (with Fenestella prisca). Taberg furwace.

$10+$ Shaje (with Atrypa plicatella). Taberg furuace.

105 Shale (with Beyrichia and Tentaculites). T'aberg furnace.

106 OOLitic IRON ORE (with fragments of fossils). Stebbins's creek.

107 do

108 do

109 do

110 do

111 do

112 do

113 do

Stcbbins's creels.

(with fragments of fossils). Near Clintun village. Quarry north of Clinton village.

Haumond's quarry. (with Phenoptera constellata). Wolcott, Wayne. (with sulphate of baryta). Wolcott. (with Pentamerus oblongus). Donnelly's quarry.

114 Limestone (with fragments of fossils). Near Martville, Cayuga.

115 Sandstone (with iron pyrites). Stebbins's quarry, Clinton, Oneida.

116 Shaly Sandstone (with casts of Leptena). Near Blackstone's quarry.

117 Ferruginous sandstone (with cast of L. subplana). Near Clinton. 
Names.

Associated fossils, and localities.

118 Shaly sandstone (with L. rugosa). Near Tippler's quarry.

119 Siliceovs limestone (with L. rugosa). Munger's quarry.

120 Siliceous limestone (with L. rugosa). Oneida lake.

121 Siliceous and shaly limestone. Swift's creek, Oneida.

122 Shaliy limestone (with L. corrugata and Pyrenomaus cuneatus). Clinton, Oneida.

123 Shaly sandstone (with Avicula emasculata). Munger's quarry.

124 do (with imperfect fossils). Gaylord's quarry, near Utica.

$125-127$ do (with Rusophycus bilobatus). Gaylord and Nor* ton's quarry, New-Hartford.

128 do (with B.palmata). Gaylord and Norton's quarry.

129 do (with roots of marine plants).

130 Gray sanìstone (with concretions).

131 Shaly sandstone (with fucoidal markings). Vanhornsville, Herkimer.

132 Shaly sandistone. Stark, Herkimer.

$133 \& 134$ Siztceous Limestone (with sulphate of strontian in crystals). Stark, Herkimer.

135 Grysum. Stark.

136 Dark-colored Gypsum. Stark.

137 Sandstone and grpsun. Stark.

$138 \& 139$ Shale with gXpsum. Stark.

10. NIAGARA GROUP.

TLEYENTII TABLE CASY.

1 Strale (with Phacops limulurus). Lockport, Niagara.

2 do (with impression of $P$. limulurus). Lockport.

3 do (with Homalonotus delphinocephalus). Iockport.

4 do (with Dictyonema retiformis). Luckport.

5 do (with Caryocrinus ornatus). Lockport.

6,7 do (with Spirifer niagarensis). Loekport. 
Names.

Associated fossils, and localities.

8 SHALE;(with Leptcena subplana). Lockport.

9 do (with Atrypa nitida). Lockport.

10 Limestone composed of crinoidal joints. Lockport.

11 ANHYDROUS GYPSUM.

12 White gYPSUM (or alabaster). Lockport.

13 Encrinal timestone (polished). Lockport.

14 Limestone (with crystallized coral). Luockport.

15 Siliceous limestone. Niagara falls.

16 DaRK limestone (with Cytherina). Wayne county.

17, 18 Dark gituminous limestone. Wayne.

19 Pearl spar. Lockport, Niagara.

20 Dogtooth spar. Lockport.

21 Pearl sfar and dogtooth spar. Lockport.

22 Dogtooth spar. Lockport;

23 Limestone (with small geodes of pearl spar). Lockport.

24 do (with galena, sulphuret of lead). Rochestcr.

25 do (with zine blende). Niagara falls.

26 do (with crystals of zinc blende). Niagara falls.

27, 28 Fragments of Orthocera. Wayne county.

29, 30 Sulphate of strontian. Lockport.

31 Pearl spar with dogtooth spar. Luockport.

32 Dark limestone (with cavities). Barre, Orleans.

33 Dark limestone (upper part of rock). Niagara falls.

34 Encrinal himestone. Niagara falls.

35 LIMEstone (with Cladopora seriata). Lockport.

36 Dari limestone. Near Lenox, Madison.

37 Brecciated limestone. Oneida county.

38 Brecciated himestone. Ham's quarry.

39 - 42 Conglomerate limestone.

43 Conglomerate limestone. Ham and Bigelow's quarries. 
NINTH WALL CASE.

Names. Associated fossils, and localities.

44 Shale (with nodules of gypsum replacing Caryocrinus ornatus). Lockport, Niagara county.

45 do (with Orthoceras undulatus). Lockport.

46 do (with Dictyonema retiformis). Lockport.

47 do (with Leptrana striata). Lockport.

48 do (with L. rugosa and L. striata). Lockport.

49 do (with Orthis elegantula). Lockport.

50 do (with O.testudinaria and L. rugosa). Lockport.

51 do (with $O$. testudinaria). Lockport.

52 do (with Bumastis barriensis). Lookport.

53 do (with fragments of various fossils). Lockport.

54 do (with O.testudinaria and L. rugosa). Lockport.

55 do (with Avicula emacerata). Lockport.

56 do (with Leptana subplana). Loekport.

57 Encrinal limestone. Near Lewiston, Niagara.

58 Shale (with Caryocrinus ornatus, and cavities filled with calcareous spar). Lockport.

59 Limestone (with crinoidal columns). Luckport.

60 Astrocerium venustum (polished specimen from the original excavation of the Erie canal, 1825). Lockport.

61 Lrmestone (with striated surface, lignilite). Luockport.

62 Limestone (with Atrypa reticularis). Lockport.

63 Brown bituminous limestone. Rochester.

64 Limestone (with Trematopora ostiolata). Rochester.

65 do (with Atrypa neglecta). Rochester.

66 do (with vertically striated surface, lignilites). Lockport.

67 Brecciated limestone. Near Rochester.

68 Wụte GұPSUM (alabaster, polished specimens). Lockport.

69 Geode of Dogtooth spar. Lockport.

70 Encrinal limestone. Lockport.

71 Encrinai limestone (polished specimen). Lockport.

72 Limestone (with dogtooth and pearl spar). Lockport. 
Names.

Associated fossils, and localities.

73 Limestone (with dogtooth spar). Lockport.

74 do (with pearl spar). Lockport.

75 do (with geode of dogtooth spar). Lockport.

76 Sulphate of strontian. Lockport.

77 Limestone (with pearl spar and dogtooth spar). Lockport.

78 do (with geode of pearl spar with selenite). Rochester.

79 Anhydrous grpsum. Lockport.

80 Sulphate of strontian. Lockport.

81, 82 Limestone (with pearl spar). Lockport.

83 Limestone (with geade filled with bituminous coal). Lockport.

84 do (with dogtooth spar). Lockport.

85 do (with pearl spar and dogtooth spar). Lockport.

86,87 do (with dogtouth spar). Lockport.

88 Pearl spar (a geode). Lockport.

89 Pearl spar and dogtooth spar. Lockport.

90 Pearl spar (rosezcolored). Lockport.

91 Limestone (with rose-colored pearl spar and dogtooth spar). Lockport.

92 do (with dogtooth spar and sulphate of strontian). Lockport.

93 do (with dogtooth spar). Lockport.

94 PEARI spar in geode of limestone. Lockport.

95 Dogtooth SFAR, Lockport.

96 Dogtooth SP.AR and PEARL SPAR (replacing Astrocerium venustum).

97 Brown spar. Lockport. Lockport.

98 Limestone (with Euomphalus hemispherica). Lockport.

99 Concretionary Limestone. North of Manchester.

100 Limestone (with concretionary nodes upon surface). Niagara falls.

101 GEODE (with pearl spar and erystals of sulphate of strontian). First excavation of Erie canal, 1825. Lockport.

102 Limestone (a concretion). Near Skonondoa, Oneida.

103 Darr bituminous limestone. Between Lenox basin and Canastota.

104 Limestone (with cavity showing lamina of growth of Astrocerium venustum). Rochester.

105 Compact unmestone (with Cytherina). Ham's quarry, Cayuga: 
Names.

Associated fossils, and localities.

106 Concretionary limestone. Four miles north of Manchester.

107, 108 Compact Dari-colored limestone. Wolcott. Wayne.

109 Linestone (with Cladopara seriata). Sweden, Monroe.

110 Limestone (with Diplophyllum caspitosum). Lockport.

1,11 Compact linestone (with Cytherina). Butler, Wayne.

112 LiMestone (with cavities lined with crystals of calcareous spar).

113 Shaly Limestone (with Spirifer crispus and minute cytherina).

114 Conpact brown limestone. Rochester.

115 Conpact limestone. North of Manchester, Ontario.

116 Concretionary uimestone. Steel's creek, Herkimer.

117 Brecciated limestone. Steel's creek.

118 Limestone (with surface markings). Ham's quarry, Cayuga.

119 Reddish conpact Limestone. Steel's creel.

120 Concretionary limestone. Near Skonondoa, Oneida. 121 - 124 Brecciated limestone. Near Skonondoa.

125 Concretionary limestone. Near Skonondoa.

\section{ONONDAGA-SALT GROUP.}

TWELFT⿱R TABLE CASE,

1 Compact red shale. Cruger's mill, Herkimor county.

2 Variegated shale. Chittenango, Madison.

3 Red shale. Between Sauquoit preek and Paris hill, Oneida.

4 Variegated shale. Baldwinsville, Onondaga.

5 Red shale (with green spots). Baldwinsville.

6 Red shale (with green spots): Oneidacastle, Oneida.

7 Greenish gray shale. North of Chittenango village.

8 Grey shale. South of Port Byron, Cayuga.

9 Green shale. Churchville, Manroe.

10 GrEEN SHALE (with seams of gypsum): Near Lake Sodom,

11 Red selentite (with green shale). Clyde, Wayne. 
Names.

Associated fossils, and localities.

12 Red GYPsum (with green shale). Lake Sodom.

13 Compact Gypsum (with green shale). Monroe county.

14 GREY shale (with cavities of crystals). Near Lake Sodom.

15 do (with remains of Cytherina). Near Peru, Livingston.

16 do (with cavities of crystals). Near Lake Sodom.

17 Calcareous infintrations between layers of gypsum. Allen's creek.

18 Gypseous Marl. Newark, Wayne.

19, 20 Grypum. Allen's creek, Monroe.

21 Gypseous marl (with seams of fibrous gypsum). Monroe county.

2.2 Selenite. Nine-mile creek.

$23,24 \mathrm{do}$ (with fragments of marl enclosed). Newark, Wayne.

25 do (with fragments of marl enclosed). Camillus, Onondaga.

26 Gypseous Marz (with marks of pseudomorphic crystals of common salt). South of Syracuse, Onondaga.

27 do (with ditto). Hill southeast of Chittenango, Madison.

28,29 do (with ditto). Bull's quarry, Isenox, Madison.

30 do (with ditto). Kelby's quarry, Lenox.

31 Pseudomorphic crystals of common salt. South of Syracuse.

32 do do Bull's quarry.

33 do do Ninc-mile creek.

34 Gray shale. Bull's quarry, Lenox.

35 do (with obscure fossils). Near Bellisle, Onondaga.

36, 37 do (with impressions of tossil shells). Bull's quarry.

38 do (with obscure fossil shells). Bull's quarry.

39 do (with plant-like impressions). Bull's quarry.

40 do (with some plant-like impressions). Bull's quarry.

41, 42 Serpentine. Near Syracuse.

$43-46$ Calcareous serpentine. Near Syracuse.

47 Serpentine (with crystals of calcareous spar). Near Syracuse.

48 Serpentine. Syracuse.

49 Gypseous marl. Nine-mile creek.

50 Black gypseous mart (with selenite). South of Bellisle.

51 Nodule of gypsum and mart. South of Chittenango. 
Names,

Associated fossils, and localities.

52, 53 Selenire (with black gypseous marl). Springport, Cayuga.

54 Dari shale (with surface covered with black selenite). Port Byron.

55 GRAY GYPSEOUS MARL (with spherical cavities containing concretions). Lenoz, Madison.

56 Impure nimestone (with spherical cavities)

57 Hydraulic limestone. Throopsrille, Cayuga.

58 Ash-CoLored HXDRAULIC LIMestone (with remains of Streptelasma). Blackrock, Erie.

59 Hydraulic limestonè (with lignilites). Canescraga creek.

60,61 do (with lignilites). Onondaga.

62,63 do (with lignilites). Canescraga creek.

64 Vermitcular immeatont: Near Syracuse.

65 Hydraulic timesione (with head of Eurypterus remipes). Oriskany.

TENTH WALL CAST.

66 Variegrated red Mari. Near Oneidacastie.

67 Red marl (with green spots). Chittenango, Madison.

68 Red shale. Between Sauquoit creek and Paris hill, Oneida.

69 Green shale. Between Sauquoit creek and Paris hill.

70 Gray shale. Near Chittenango, Madison.

71 Gray shale. Allen's creek, Monroe.

72 Gray gypseous marlo. Orleans county.

73 Gray gypseous siralri. Niar Churchville, Monroe.

74 Gypseous marl.

Allen's creek.

75 Gypseous MarL.

lockville, Wayne.

76 Friable sandstone.

Near Paris hilJ, Oneida.

77, 78 Gypseous shale.

Near Paris hill.

79 Gyfseous marl.

Allen's creek.

80 Gypseous marl (with seams of fibrous gypsum). Monroe county. 81, 82 Greenish marl (with fibrous gypsum). Jordan, Onondaga.

83 Red gxpsum (with gypseous marl). Jordan.

84 Red Grysum (nodules). Newark, Wayne.

85, 86 Gypseous marl. Clyde, Wayne. 
Names.

Associates and localities.

87 MarL (with red selenite). Newark.

88 Gypseous marl. Near Port Gibson, Monroe.

89 Arglldaceous limestone. Near Churchville, Monroe.

90 Selenite (with decomposing iron pyrites). Newark, Wayne.

91 Gypsum.

Newark.

92 Grpsum.

Allen's creek.

93, 94, 95 Grpsum. Leroy, Genesee.

96 Gypsum (nodule). Monroe county.

97 Granưlar gypsum. Allen's creek.

98 Gypseous marl (with selenite). South of Bellisle, Onondaga.

99 Grey grPseous Marl.

100 Grey argillaceous himestone.

101 Gypseous shale. Near Waterville, Oneida.

102 Compact limestone (with minute vermicular eavities). Waterville.

103 Confact limestone (with minute cavities filled with coneretions). Near Waterville.

104 Grey shale (with cavities made by crystals), Near Jordan lake.

105 Dark shaly limestong. Near Waterville.

106 Compact limestone. Near Waterville.

107 Shacy limestone, Near Waterville.

108 Grey shale.

109 GyPsEous MarL (with selenite). Newark, Wayne.

110, 111 Gypseous MarL. Near Camillus, Onondaga.

112 Selenite, Near Camillus.

113 GYPSEOUs MARL (with selenite and marks of pseudomorphic crystals).

Near Camillus.

114 Gypseod maru (with marks of pseudomorphic crystals). Camillus. 115, 116, 117 Pseddomorphic crystats of common salt. Camillus. 118 Pseudomorphic crystals (with imperfect fossil shells on shale). Lenox, Madison.

119 IMPERFEOT PSEUDOMORPHIC CRYSTALS. Brown's quartý.

120 Gxpseods shale (with marks of pseudomorphic crystals). Bellisle:

121 Gypseous shales (with marks of pseudomorphic crystals). Lenox. 
Names. Associates and localities.

122 Gypseous shales. Near Camillus.

123 Psevdomorphic crystal of common salt. Near Camillus.

124 Grpseous shale (with pseudomorphic erystals). Near Camillus.

125 Dark-colored shale.

126 Grey shale. Near Bellisle, Onondaga.

127, 128 Dark shale (with impression of marine plants). Lenox.

129 Grey shale. Chittenango, Madison.

130, 131, 132 Serpentine and limestone. Syracuse.

133 Serpentine and limestone (polished). Syracuse.

134 Serpentine and timestone. Syracuse.

135 Serpentine (polished). Syracuse.

136 - 143 Serpentine. Syracuse.

144 Serpentine and linestone. Syracuse.

145 Sulphate of strontian in gray marl. Syracuse.

146 Gray Marl (with erystals of sulphate of strontian). Syracuse.

147 Gray gypsemous Mard. Lenox, Madison.

148 Gray shaLe (with impressions of fossil shells). Lenox.

149 Shale (with obscure fossil shells). Lenox.

150 Vestcular limestone. Onondaga-hollow.

$151-153$ do Lenox.

$154 \& 155$ do Port Byron.

156 do Near Syracuse.

157 Dark shale. Cherryvalley, Otsego.

158 Limestone. Chittenango, Madison.

159 Limestone (with drusy cavities). Oriskany-falls, Oneida.

160 Gypseous marl. Cherryvalley, Otsego.

161 Gray shaly limestone. Chittenango, Madison.

162 Gypseous Marl (with calcareous incrustations). Monroe county.

163 Hydraulic limestone (with carities).

164, 165 Gray gypseods shale. Chittenango.

166 Gypseous mard.

167 Nodute of GYPsum. Near Jamesville, Onondaga.

168 Gypseods Mari (with seams of fibrous gypsum). Phelps, Ontario. 
Names.

Associates and localities.

169 Selenite (with compact gypsum). Phelps.

170, 171, 172 Gypseous Marl (with selenite). Springport, Cayuga.

173 Limestone above gypsum beds. Jamesville, Onondaga.

174 Gxpseous marl (with nodules of gypsum). South of Chittenango.

175,176 Compact gypseous Mari.

177 Graver cemented by gypsum. Boring at Salina, Onondaga.

178 Gypseous shale.

179 CoMpact LIMESTone (with vesicular cavities filled with crystalline matter). Near Lanark's mill.

180 Сompact Limestone (with zine blendẹ). Near Lanark's mill.

181 Compact limestone (with irregular cavities). Near Lanark's mill.

182, 183 Hydraulic Limestone (with lignilites). Near Lanark's mill.

184 Hydraulic limestone (with obscure fossils). Byron, Genesee.

185, 186 Hydraduic liniestone (with cherty nodules). Near Camillus.

187 Compact shaly limestone. Phelps, Ontario.

188 CrystalLized Gysum (deposits from the salt-vats, ten specimens). Syracuse, Onondaga.

189 Calcareous formation having oolitic structure (deposits from saltvats, four specimens). Syracuse.

190 Oolite (deposits from salt-vats, three specimens). Syracuse.

191 Calcareous mass showing oolitic and pitted structure (deposits from salt-vats). Syracuse.

UPPER PART OF THE ONONDAGA-SALT GRODP CONTINUED IN TEE WALL CASE OF THE WATER-LIME GROUP.

1, 2, 3 Hydraudic limestone. Allen's creek.

4 Hydraulic limestone. Clarence-hollow.

$5-8$ do Allen's creek.

9 do Monroe county.

10 do Caledonia, Livingston.

11 do Near Lewiston, Niagara.

12 do Allen's creek.

$13 \& 14$ do Williamsville, Erie.

$15 \& 16$ do Street farm, Caledonia, Livingston 
Names

Associates and localities.

17 Hydraulic limestone. Allen's creek.

18 do

19 do

20 do

21 do

22 do

23 do

24

do,

Street farm, Caledonia.

Phelps, Ontario.

One mile east of Vienna, Oneida.

Near Vienna.

(with sulphate of strontian). Street farm.

Phelps, Ontario.

25 Shaly hydraUlic limestone.

26 Hylraulic limestone (with nodule of chert). Marcellus, Onondaga.

27, 28 Fydraduic limestone (with erystalline nodules). Lanark's mills.

29 Shaly hydRadLic Limestone.

30 Dark-colored hydraUlic limestone. Marcellus, Onondaga.

31 Hydraulic limestone. Cherryvalley, Otsego.

32 Hydraulic limestóne (dark colored). Near Marcellus.

33 Shaly hydratulic limestone. Near Deansville, Oneida.

34 Shaly hydraulic limestone (dark colored). Near Deanstille.

35 Hydraulic limestone. Onondaga valley.

36 - 41 Hydraulic limestone (with fluor spar). Manlius, Onondaga.

42 Hydraulic limestone. Onondaga valley.

43

do

Cherryvalley, Otsego.

44

do

Phelps, Ontario.

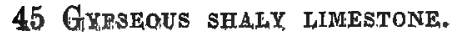

46 Hydraulic limestone. Near Lanark's mills.

47 do (shaly). Oriskany falls, Oneida.

48 do Chittenango, Madison.

49 do Near Manlius, Onondaga.

50 do (with anhydrite).

51 Vesicular limestone. Near Syracuse.

52 Hydraulic limestone. East Steel's creek, Herkimer.

53

do

(upper part shaly, with crystals of baryta; three specimens). Phelps, Ontario. 
Names.

Associates and localities.

55 Hydraulic limestone (with lingulæ). Cherryvaliey, Otsego.

$\begin{array}{lll}56 \& 57 & \text { do } & \begin{array}{c}\text { (vesicular). Oriskany, Oneida. } \\ 58\end{array} \\ 59 & \begin{array}{c}\text { do } \\ \text { do }\end{array} & \begin{array}{c}\text { (with cavities filled with crystalline matter). } \\ \text { Near Jamesville. }\end{array} \\ 60 \& 61 & \text { do } & \begin{array}{c}\text { (with crystals of magnesian carbonate of lime). } \\ \text { Near Marcellus, Onondaga. }\end{array} \\ 62 & \text { do } & \text { (with nodule of chert). Near Marcellus. } \\ 63 & \text { do } & \text { (with gypsum). Near Syracuse. }\end{array}$

NEW-YORK SYSTEM (CoNTINUED).

Name of Group.

13. WATERLIME GROUP.

14. Pentamerus limestone. Sixteenth table case. - - - 49

15. Catskill shaly limestone. Seventeenth table case. - - 42

16. OrisKanY SANDSTONE. Eighteenth table case. - - - 45

17. CAUDA-GALLI GRIT.

18. SCHOHARIE GRIT.

19. OnONDAGa Limestone.

20. Corniferots limestone. Twenty-second table case. - $\quad 54$

21. Marcellos shale.

22. Hamilton group.

22. Hamilton grodp.

23. TUlLt LIMESTONE.

24. Genesee slate.

25. Portage group.

26. Ithaca grodp.

27. Chemung grodp.

28. CAtskill Grodp.

Conglomerate.

Carboniferots system (of Pennsylvania). 33d table case. 33

Miscellaneous.

Miscellaneods.

Miscellaneous.

Miscellaneous.
Nineteenth table case. - - - 23

Twentieth table case. - - - 41

Twenty-first table case. - - $\quad-67$

Twenty-third table case. - - 55

Twenty-fourth table case. - - 62

Twenty-fifth table case. - - $\quad-74$

Twenty-sixth table case. - - $\quad 29$

Twenty-seventh table case. - - 36

Twenty-eighth table case. - - - 32

Twenty-ninth table case. - - 50

Thirtieth table case. - - - 50

Thirty-first table case. - - $\quad 55$

Thirty-second table case. - - 16

Thirty-fourth table case. - - - 49

Thirty-fifth table case. - - - - 66

Thirty-sixth table case. - - - 77

Thirty-seventh table case. - - - 75

Fifteen wall cases unarranged specimens. 



\section{PAL君0NTOLOGY OF NEW-YORK,}

BY JAMES HALL. 



\title{
PALE0NT0L0GY.
}

\section{POTSDAM SANDSTONE,}

\author{
Name. Locality. In the
}

11 Scolithus linearis (1 specimen). - * - - - - Cabinet. BRACHIOPODA.

21 Lingula prima(1).

32 Lingula antiqua(2).
Keeseville, Essex county. Cabinet. Hammond, St.Lawrence. Cabinet.

\section{OALCIFEROUS SANDSTONE.}

PLANTS.

41 Pal agophycus tubularis(4). Canajobarie and Fortplain. Cabinet.

52 Paldoophycus irregdlaris. Near Keeseville. - - - -

61 Buthotrephis aniridata(1). Chazy, Clinton. - - Cabinet.

BRACHIOPODA,

73 Lingula acuminata (in a boulder).

GASTEROPODA,

81 Euomfhalus uniangulatus (plaster cast). - - - Cabinet.

91 Maclurea sordida(2), Mohawk valley. - - Cabinet.

102 Maclurea matutina, Canajoharie, Montgomery.

111 Ophlleta levata(2), Near Fortplain. - - Cabinet.

122 Ophileta complanata (1). Mohawk valley. - - Cabinet.

131 Turbo dilucula. Middleville and Littlefalls.

142 Turbo? obscura(1). Fortplain, Montgomery. - Cabinet.

151 Plevrotomaria? turgida. Saratoga county. 


\section{CEPHALOPODA.}

161 Orthoceras Primigenium(1). Near Fortplain. - - Cabinet. 172 Orthoceras laqueatum(1). - - . - - - Cabinet.

\section{CHAZY LIMESTONE.}

\section{CORALS.}

181 Retepora incepta(1).

192 Retepora Gracilis.

201 Gorgonia? ASPERA.

211 Stictopora fenestrata(1).

222 Stictopora glomerata.

231 Streptelasma expansa(2).

241 Chatetes ?(1).
Chazy, Clinton. - - Cabinet.

Chazy.

Chazy. - - - Cabinet.

Granville (Vermont).

Chazy. - - - Cabinet.

Chazy. - - - Cabinet.

\section{CRINOIDEA.}

251 Actinocrinus tenuiradiatus(1). Chazy. - - - Cabinet.

26 2 ACtinocrinus? Near Chazy.

271 Asterias?

\section{BRACHIOPODA,}

281 Lept anna PLicifera(1).

292 Leptexa incrassata(3).

303 Left fena fasciata(1).

31 1. Orthis costalis(1).

321 Atrypa dubia(1).

332 Atrypa acutirostra.

343 Atrupa plena(1).

354 Atrupa plicifera(1).

365 Atrupa altilis(1).

371 OrbicUla? deFormata.

381 Metoptoma? dubia.

CRUSTACEA.

391 ILL正US ARCtURUS(1).

402 IlLagnUS CRASSICAUda? (1).
Chazy. - - - Cabinet. Chazy. - - : Cabinet. Chazy. - - - Cabinet.

Ohazy. - - - Cabinet. Chazy. - - - - Chazy. - - - Cabinet. Chazy. - - - Cabinet. Chazy. - - - Cabinet. Chazy.

Chazy. - - - Cabinet. Chazy. - - - Cabinet. Chazy. - - - Cabinet. 
Names.

41 Asaphus? obtusus(1).

422 Asaphus marginalis.

431 Isotelus gitas.s? (1).

442 Isotelus canalis( 1 .

451 Ceraurus?
Localities.

Chazy. - - - Cabinet.

Chazy. - - - -

Chazy. - - - Cabinet.

Chazy. - - - Cabinet.

Chazy. - - - Cabinet.

\section{GASTEROPODA.}

463 Maclitrea magna(8).

471 Scalites angulatus(2).

481 Raphistoma striata(1).

492 Raphistoma staminea(1).

503 Raphistoma planistria(1).

514 Raphistoma planistria, var, parva. Chazy.

522 Pleurotomaria biangulata (fragment). Chazy. - - Cabinet.

533 Pleurotomaria

544 Pleurotomaria antiqtata(1).

551 Capulus auriformis.

561 Murchisonia abbreviata.

571 Bucania sulcatina(1).

582 Bucania rotundata (a cast).

Chazy. - - - Cabinet. Chazy. - - - Cabinet. Chary. - - - Cabinet. Chazy. - - - Cabinet. Chazy. - - - Cabinet. Chazy. - - - Chazy. - - - Cabinet. Galway, Saratoga. Chazy. Chazy. - - - Cabinet. Chazy. - - - CEPHALOPODA.

593 Orthoceras rectiannulatum(1). Chazy. - - - Cabinet.

604 Orthoceras sUbarcuatum. Chazy. - - - 615 Orthoceras tenuiseptum(1). Chazy. - - - Cabinet. 626 Orthoceras bilineatum. 637 ORTHOCERAS MONILIFORME. Near Albany.

Chazy.

\section{BIRDSEYE LIMESTONE.}

\section{PLANTS ?}

641 Phytopsis tobdosum(6). Mohawk valley. - Cabinet.

652 Phytopsis cellulosum(2). Watertown \& Mohawk valley; Cabinet. ACEPHALA.

661 Modiola? OBTUSA(1). Watertown, Jefferson. Cabinet. 


$$
\text { Names. GASTEROPODA. Localities. }
$$

672 Murchisonia? angustata.

683 Murchisonia ventrigcosa(1). Watertown, Jefferson. Cabinet.

694 Murchisonia perangdiata.

Watertown. - -

705 Murchisonia varicosa(1).

Watertown. - - Cabinet.

711 Natica? -

Watertown.

725 Pleurotomaria? nucleolata. Watertow.

736 Pleqrotomaria quadricarinata(1). Watertown. - Cabinet.

747 Pleurotomaria umbilicata(4). Watertown. - - - Cabinet.

758 Pleurotomaria? nodulosa(1). Watertown. - - Cabinet.

769 Pleurotomaria? obsoleta(1). Watertown. - - Cabinet. CRUSTACEA.

771 Cytherina (fragments).

Watertown. - - Cabinet.

\section{CEPHALOPODA.}

788 Orthoceras multicameratum(1). Watertown. - - Cabinet.

799 Orthoceras recticameratum. Watertown.

\section{CORALS OF THE BIRDSEYE AND BLACK-RIVER LIMESTONES.}

801 Columaria alveolata(3).

811 Stromatocerium hugosum.

822 CheteTes LyCOPERDon?

832 Streptelasma profunda $(6)$.

843 Stictorora Labtrinthica(1).

854 Stictopora ramosa.
Mohawk valley, - - Cabinet. Watertown, Chazy, \&c.

Watertown.

East-Canada creek. Cabinet. Chazy. - - - Cabinet. Watertown. - -

\section{CEPHALOPODA.}

861 Lituites undata (plaster cast). Watertown. - - - Cabinet.

872 Lituites convolvans?(1).

881 Gonioceras anceps $(1)$.

891 Ormoceras tenuifilum(4).

902 Ormoceras tenuifllum, var. Distans. Watertown.

913 Ormoceras? gracile.

921 Endoceras subcentrale.

932 Endoceras longissimum.

943 Endoceras multitubulatum.

Watertown. Watertown.

Watertown.
Watertown. - - Cabinet. Watertown. - - Cabinet. Watertown. - - Cabinet. 
Names.

954 Endoceras gemelitiparum.

9610 Orthoceras fusiforme(1).
Localities.

Henderson's bay.

Watertown.

- Cabinet.

\section{TRENTON LIMESTONE.}

\section{PLANTS.}

972 Buthotrephis gracilis(1). Jacksonburgh, Herkimer.Cabinet.

983 Buthotrephis succulens.

993 Palaophycus rugosus(1).

100 4. Paleophycus simiplex(1).

\section{CORALS.}

1012 Chetetes LyCoperdon $(9)$.

1023 Chetetes rugosus.

1034 Chatetes columararis.

1041 Receptacultes neptunit.

1053 Streptelasma corniculumi(1).

1064 Streptelasma crassa.

1075 Streptelasma multilamellosa(1). Watertown. - - Cabinet.

1086 Streptelasma parvula(1).

1091 Porites? vetusta.

1101 - - CYATHIFORMIS.

1111 Escharopora reCta $(1)$.

1122 Escharopora RECTA, var. Nodosa.

1135 Stictopora? aCUTA.

1146 Stictopora elegantula.

1152 Gorgonia perantiqua.

1161 Aulopora arachnoIdea.

1171 Alecto inflata.

1181 Intricaria? RETICULATA(1).

1193 RETEPORA? FoliACEA.

1201 Stelitpora antheloIdea.

1211 Graptolites amplexicadle.
Glensfalls.

Below Prospect hill. Cabinet. Middleville, Herkimer. Cabinet.

Various places. - - Cabinet.

Middleville.

Sugar river.

Carlisle (Pennsylvania).

Middleville. - - Cabinet. Near Middleville.

Middleville. - - Cabinet.

Carlisle (Pennsylvania).

Jacksonburgh. - Cabinet.

Lowville, Lewis. -

Middleville. - -

Near Middleville.

Ohio and Kentucky.

Trenton falls, Oneida.

Watertown. - - Calinet.

Lowville.

Lowville.

Trenton falls, etc. Watertown. 
NEW-YORK STATE COLLECTION.

\section{CRINOIDEA. \\ Names. $\quad$ Localities.}

1221 Schizocrinus nodosus(3). Glensfalls and Middleville.Cabinet.

1231 Poteriocrinus alternatus (fragment). - - - - - -

1242 Potertocrinus gracilis. Middleville.

1251 Scyphocrinus heterocostalis. Middleville.

1262 Schizocrinus —

1271 Echino-encrinites anatiformis. Turin, Lewis. - - -

1282 Asterias matutina. Trenton falls.

1291 Tentaculites? flexuosa. Lowville.

\section{BRACHIOPODA.}

1304 LingULA atTEnUATA?

1315 lingula ricinirormis(2).

1326 Lingula aqualis(1).

1337 Lingula quadrata(1).

1348 LINGULA ELONGata.

1359 Lingula curta.

13610 Lingula obtusa(1).

137 11. Lingula crassa(1).

1382 Orbicula? filosa(1).

1393 Orbicula lamellosa.
Middleville, etc.

Middleville. - - Cabinet.

Middleville, etc. - Cabinet.

Lewis county. - - Cabinet.

Lewis county.

Middleville, etc.

Middleville. - - Cabinet.

. - - - - - Cabinet.

Middleville. - - Cabinet.

Middleville.

1404 Orbicula (Trematis) terminalis(1). Middleville. - Cabinet.

1414 Leptena alternata(8). Middleville and Watertown.Cabinet.

1425 Leptena camerata(1).

Trenton falls. - - Cabinet.

1436 Leptena deltoidea(2). Middleville and Trenton falls.Cabinet.

1447 Leptena tenuistriata(1).

1458 Lepterna alternistriata.

1469 Leptena sericea(4).

14710 Leptana filitexta(2).

14811 Leptena planumbona.

14912 Leptana DeFlecta.

15013 Leptema recta.

15114 LEPTIANA PLANOCONVEXA.

15215 Leptena tenUilineata.
Middleville, etc. - Cabinet.

Cincinnati, Ohio. -

Middleville and Lowville.Cabinet.

Middleville. - - Cabinet.

Cincinnati, Ohio. -

Mineral point (Wisconsin).

Mineral point.

Cincinnati (Ohio). - 
Names.

15316 Lept aena subtenta.

1552 Orthis testudinaria(3).

1563 Orthis sUbæquata.

1585 Orthis disparilis.

1596 Orthis PERVETA.

1607 OrThIS RQUIVALVIs.

1618 Orthis Fissicosta.

1629 Orthis tricenaria.

16310 Orthis plicatella.

16411 Orthis pectinelda(3).
15417 Lept

1574 Orthis bella-RUgosa.

Localities.

Trenton falls?

Middleville. - - Cabinet.

Mineral point (Wisconsin).

Mineral point (Wisconsin).

Mineral point.

Middleville?

Near Cincinnati (Ohio).

Middleville. - -

Sugar river, Lewis. Cabinet.

16512 Orthis pectinelia, var. semiovalis(1). Turin, ete. Cabinet.

16613 Orthis INSCULPTA.

Watertown, etc.

16714 Orthis Dichotoma.

Cincinnati (Ohio).

16815 ORTHIS sUbQUADRATA.

Cincinnati and Oxford (Ohio).

16916 Orthis occidentalis(3).

17017 Orthis sindata.

17118 Orthis subjugata.

1721 Delthyris hynx(7).

1736 Atrypa extans(1).

1747 Atrupa NUCLEUS.

1758 Atrypa cuspidata(3).

1769 Atripa bisulcata.

17710 Atrypa DefLeCta.

17811 Atrypa recurvirostra(1).

17912 Atrypa exigua.

Cincinnati and Oxford, Cabinet.

Cincinnati and Oxford.

Cincinnati and Oxford.

Trenton falls, Oneida. Cabinet. Watertown, Jefferson. Cabinet. Middleville, Herkimer.

Lowville, Lewis. - Cabinet. Adams, Jefferson.

Near Martinsburgh, Lewis.

Martinsburgh. - Cabinet. Lowville.

18013 Atrypa modesta(1).

Cincinnati and Oxford (Ohio).Cabinet.

18114 Atrypa circulus.

Middleville, Herkimer.

18215 Atriypa ambigua.

18316 Atrypa hemiplicata(3).

18417 Atrupa

Middleville.

Middleville. - - Cabinet.

Middleville.

18518 Atrypa sUbTRIGONALIS.

Turin, Lewis. 
Names.

18619 Atrupa inCREbESCENS(4).

18720 Atrupa dentata.

18821 ATRYPA SORDIDA.

\section{ACEPHALA.}

1891 Nucula levata(3).

1902 Nucula poststriata.

1911 Teluinomya nasuta (1 and a cast). Middleville. - - Cabinet.

1922 Tellinomya sanguinolaroidea (plaster east). - - Cabinet.

1933 Tellinomya gibbosa ( 1 and a plaster cast). Middleville.Cabinet.

1944 Tellinonya dubia(2).

Middleville, etc. - Cabinet.

1955 Tellinomya anatiniformis(1). Watertown. - - Cabinet.

1961 Cardiomorpha vetusta.

Middleville.

1971 Edmondia ventricosa(2).

1982 Edmondia subtruncata.

1993 Edmondia? subangulata(1).

2001 Modiolopsis Mytiloides(1).

2012 Modiolopsis parallela.

2023 Modiolopsis faba(3).

2034 Modiolopsis nastutus(1).

2045 Modioloysis arcuatus.

2056 Moniolopsis subspatulatus.

2067 Modiolopsis latus

2078 Modiolopsis carinatus.

2089 Modiolofsis aviculoides.

20910 Modiolopsis? trentonensis.

2101 Avicula trentonenisis(2).

2112 Avicula eldiptica.

2121 Ambonychia belisstriata (plaster cast). Trenton falls. Cabinet. 2132 Ambonychia orbicularis(1). Watertown. - - Cabinet. 2143 Ambonxchia amygdalina (plaster cast), Adams. - Cabinet. 2154 Ambonychia undata(1), Watertown. - - Cabinet. 2165 Ambonychia ortusa (plaster cast). Watertown. - - Cabinet. 2176 Ambonychia? Middleville.

Lowville. - - Cabinet.

Watertown. * - -

Watertown. " - Cabinet.

Middleville. - * Cabinet.

Middleville and Watertown.Cabinet.

Carlisle (Pennsylvania).Cabinet. Herkimer. - .

Watertown.

Watertown.

Middleville.

Middleville.

Middleville. - - Cabinet. Middleville. 


\section{Names.}

2181 Holopea symmetrica.

2192 Holopea obliqua(2).

2203 Holopea paludiniformis(1).
Localities.

Middleville.

Watertown. - - Cabinet.

Watertown. - - Cabinet.

2214 Holopea ventricosa (plaster cast). Middleville. - Cabinet. 22210 Pleurotomaria subtilistriata, Watertown.

22311 Pleurotomaria lenticularis(2). Watertown. - - Cabinet. 22412 Pleurotomaria rotuloides. Middleville.

22513 Pleurotomaria subconica (plaster cast). Watertown. Cabinet.

747 Pledrotomaria umbilicata. Juowille and Adams.

22614 Pletrotomaria indenta(1). Watertown. - - - Cabinet. 22715 Pleurotomaria ambigua (plaster cast). Adams. - - Cahinet. 22816 Pleurotomaria percarinata (plaster cast). Middleville.Cabinet. 2296 Murchisonia Bicincta(2). Lowville and Watertown.Cabinet

2307 Murchisonia tricarinata. Mineral point (Wisconsin).

2318 Murchisonia perangulata. Middleville. - . 2329 Murchisonia uniangutata. Middleville.

23310 Murchisonia eellicincta(2). Middleville, Turin, etc.Cabinet 23411 Murchisonia subfusiformis (1 and a cast). Lowville. Cabinet.

23512 Murchisonia vittata.

23613 Murchisonia gracilis(2).

2871 Subulites elongata(2).

2381 Carinaropsis carinata.

2392 Carinaropsis patelliformis. Middleville. - - - -

2401 Bellerophon bilobatus(2). Trenton falls \& Middleville.Cabinet.

2412 Bejlerophon bilobatus, var. acutus. Trenton falls.

2423 Bellerophon bilobatus, var. corrdgatus. Trenton falls.

2433 BUCania Expansa (plaster cast). Watertown. - - Cabinet.

2444 BUCANia BIDORSATA.

2455 Bucania punctifrons(2).

2461 Cxrtolites compressus(3).

2472 Cyrtolites trentonensis.

2483 Cyrtolites filosum (plaster cast). Watertown, - Cabinet.
Watertown. - - Middleville. - - Cabinet. Middleville. - - Cabinet.

Mohawk valley. - - - 
CEPHALOPODA.

2491 Trocholites ammonius(1).

2501 Cyrtoceras lamellosum.

2512 Cyrtoceras anNulatum.

2523 Cyrtoceras macrostomum (plaster cast). Wisconsin. Cabinet.

2534 Cyrtoceras constrictostriatum. Middleville.

2545 Cyrtoceras multicameratum. Middleville. -

2556 Cyrtoceras arcuatum.

Middleville.

2567 Cyrtoceras camurum.

2571 ONCOCERAS CONSTRICTUM(2).

25811 Orthoceras arctoliratum(2).

25912 Orthoceras teretiforme(1).

26013 Orthoceras textile.

26114 Orthoceras bilineatum(1). Middleville. - - Cabinet.

26215 Orthoceras bilineatum, var. \%. Middleville, etc.

26316 Orthoceras clathratum. Miadleville.

26417 Orthoceras vertebrale.

26518 Orthoceras anellum.

266-19 ORthoceras undulostriatum.

26720 ORTHOCERAS - -

26821 Orthoceras latianNulatum.

26922 ORTHOCERAS JUNCEUM.

27023 Orthoceras amplicameratum.

27124 Orthoceras STRIgatUM.

172 Orthoceras LaQUEATUM.

27225 Orthoceras Laqueatum, var. $u$. Middleville.

2735 Endoceras annulatum. Watertown.

2746 Endoceras protefforme, var. tenuistriatum(1). - Cabinet. 2757 Endoceras froterforne, var. tenuitextum(1). - - Cabinet.

2768 Endoceras proteiforme, var. imeolatum(1). - - Cabinet. 2779 Endoceras protetforme, var. strangulatum(1). - Cabinet. 27810 Endoceras proteiforme, var. elongatum(1). - - - Cabinet. These five varieties of ENDOCRras proteiforme were all obtained in Middleville, Herkimer county. 
Names.

27911 Endoceras arctiventrum.

28012 Endoceras angusticameratum. Middleville.

28113 Endoceras magniventrum(1). Near Middleville. - Cabinet. 28214 Endoceras magniventrum, var. Near Middleville.

28315 Endoceras approximatum.

28416 Endoceras duplicatum(1).

28517 Endoceras distans.

2861 Cameroceras trentonense.

891 ORMOCERAS TENUIFILUM?

2871 Conularia trentonemsis(1).

2882 Conularia granulata.

2893 Conularia papillata.

2904 Conularia gracile.
Localities.

Middleville.

Near Middleville.

Near Middleville. - Cabinet.

Turin, Lewis.

Middleville. - -

Turin, ete.

Middleville. - - - Cabinet.

Middleville, etc.

Near Middleville.

Near Middleville.

CRUSTACEA OF THE BIRDSEYE AND TRENTON LIMESTONES, HUDSON-RIVER GROUP, AND UTICA SLATE.

2911 Ogygia? vetusta.

2923 Asaphus extans(1).

2931 Calimene multicosta $(1)$.

402 Illemis crassicatdid.

2943 Illand trentonensis.

2954 Ill æenUs latidorsata.
Mohawk valley.

Lowville. - - - Cabinet.

Isle Lamotte. - - Cabinet.

Carlisle (Pennsylvania).-_

Hogansburgh, Franklin.

Near Watertown.

431 Isotelus gigas (several imperfect specimens).Middleville.Cabinet.

2961 Platynotus trentonensis (plaster cast). Middleville,etc.Cabinet.

2972 Calymene bechir $(4)$.

2983 Calymene senaria(2).

2991 Actdaspis trentonensis.

3002 ACIDASPIS SPINJgER.

3012 Ceraurus pleurexanthemus (fragments). Middleville. Cabinet.

3023 Ceraurus vigilans.

3034 Ceraurus? pustulosus.

3041 Phacops callicephatus.

3052 Phacors? laticaudus.
Middleville. - - Cabinet.

Middleville. - - Cabinet.

Bay of Quinty.

Mohawr valley.

Middleville. - - -

Watertown.

Middleville \& Watertown.

Turin. 
Names.

3064 Asaphus nodostriatus(1).

3071 Trinucleus concentrictus(2)

3084 Calymene

3095 Asaphus? latimarginata.

3101 Olenus asaphoides.

3112 Olends undulostriatus.

3121 Agnostus lobatts.

3131 Thaleops (Iltanns) ovatus.
Localities.

Watertown. - - Cabinet.

Loraine, etc. - - Cabinet. Canajoharie.

Watertown.

Greenwich, Washington.

Snakehill, Saratoga.

Troy, Rensselaer. -

Mineral point (Wisconsin).

\section{HUDSON-RIVER GROUP.}

\section{PLANTS.}

3141 Sphenothallus angustifolits. Mohawk valley.

3152 Sphenothallos latifolitgs(6). Schoharie. - - Cabinet.

3164 BUThOTREPHIS SUBNODOSA.

Turin, Lewis.

3175 BUTHOTREPHIS? FLEXUOSA.

Jackson, Washington.

3185 Palomophycus virgatus(1).

Union, Washington. Cabinet.

3196 PaLeophycus ?(1).

Rome, Oneida. - - Cabinet.

3201 Gordia marina.

Jackson, Washington.

CORALS.

3212 Graptolithus pristis(1).

Turin, Loraine, etc. Cabinet.

3223 Graptolithus secalinua(1).

Hoosick slate quarries.Cabinet.

3234 Graptolithus mucronatus(1).

3245 Graptolithus bicornis(2).

Near Albany. - Cabinet.

3256 Graptolithus ramosus(1). Albany. - - - Cabinet. Albany. - - - Cabinet.

3267 Graptolithus scalaris(1). West-Canada creek \& Albany.Cabinet. 3278 Graptolithus sagittarius(1). Albany. - - - Cabinet.

3289 Graptolithus tenuis(1).

32910 Graptolithus sextans(1). Albany. - - - Cabinet.

33011 Graftolithus furcatus. Albany. - - - Cabinet.

33112 Graptolithus serkatulus.

Normanskill near Albany.

33213 Graptolithus gracilis(1).

Normanskill.

Albany. - - - Cabinet. 
Names.

33314 Graptolithus Lavis.

3341 Favistelda stellata.

1012 Chatetes lycoperdon.

CRINOIDEA.

3351 Discophyllum Peltatum.

3361 Undetermined.

Near Troy, Rensselaer.

Turin, Pulaski, Loraine, etc.

3371 HetrRocrinus heterodactylus (columns on a fragment of slate). Near Rome, Oneida. Cabinet.

3382 Heterocrinus simplex.

Cincinnati (Ohio).

3393 Heterocrinus? gracilis.

Snakehill, Saratoga.

3401 Glyptocrinus decadactylus (columns on a fragment of slate). Turin, Rome, etc. - Cabinet.

1291 Tentaculites Flexuosa. Turin. - - - Cabinet.

\section{BRACHIOPODA.}

1337 Lingula quadrata(1).

1414 Lepterina alternata(4).

1469 Lepterna sericea $(1)$.

1552 Orthis testudinaria(1).

34119 Orthis? erratica(2).

34220 ORTHIS CENTRILINEATA.

18619 AtruPa increbesCens(1).

3435 Orbicula? subtruncata.

3446 Orbicula? crassa.

3457 ORBicula cerlata.

\section{ACEPHALA,}

3463 Avicula insueta(1).

3474 Avicula deitussa.

3485 Avicula? Desquamata.

3497 Ambonychia radiata(3).

3508 Ambonychia carinata.

35111 Modiolopsis modiolaris(3). Loraine, Pulaski, ete. Cabinet.

35212 Modiolopsis TRUnCATUS.

Loraine, Jefferson. - Cabinet. Pulaski, Oswego. - Cabinet. Turin, Lewis. - - Cabinet. Turin, Pulaski, etc. Cabinet. In southern counties. Cabinet. Loraine. - - -

Turin. - - - Cabinet. Loraine. - - - - Near Troy.

Near Troy.

Canajoharie. - - Cabinet. Near Rome, Oneida.

Near Troy.

Pulaski and Turin. - Cabinet. Near Rome. 
Names.

2034 Modiolopsis nasutus $(\mathbf{1})$.

35313 Modiolopsis cURta.

35414 Modiolorsis

35515 Modiolopsis anodontoides.

2023 Modiolopsis Faba.

35616 Modiolopsis? NUCULIFormis.

3571 ORTHONOTA PHOLADIS.

3582 Orthonota parallela.

3593 Orthonota contracta.

3601 Cleidophorus ptandulatus.

1902 NuCULa? POSTStriata.

3611 Lyrodesma plana.

3622 Lyrodeśma pULChELLA.

GASTEROPODA.

23613 Murchisonia gracilis.

Loraine. -

36314 Murciisonia uniangdiata, var.

22513 Pleurotomaria subconica.

abbreviata. Near Rome.

36417 Pleurotomaria? bilix(2). Pulaski.

36518 Pleurotomaria (2). Coldspring, Montgomery.Cabinet.

3662 Metoptona? Rugosa. Near Troy, Reasselaer.

2392 Carinaropsis patelitformis. Pulaski, Oswego.

3673 Carinaropsis orbictlatus.

2401 Bellerophon bilobatus.

Waterford, Saratoga.

3684 Bellerophon canceliatus(1). Turin, Loraine, etc.

3694 Crrtolites ornatus(1).

Loraine. - - - Cabinet. Turin, Pulaski, ete. Cabinet.

\section{CEPHALOPODA.}

2491 Trocholites ammonus(2). Canajoharie, Montgomery.Cabinet. 3702 Trocholites planorbiformis(1). Pulaski, Oswego. - Cabinet. 37118 Endoceras proteiforme?(3). Turin, Lewis. - Cabinet. 37226 Orthoceras Turin.

37327 Orthoceras coralliferum(2). Turin and Pulaski. - Cabinet. 37428 Orthoceras hameloosum(1). Turin, etc. - - Cabinet. 
Names.

3752 Ormoceras CRebriseptum(1).

3761 Theca? triangularis.

3779 Ambonychia mytiloidea.

3782 Schizocrinus striatus.

3793 NuCULA? DONACIFORMIS.

3806 Bucania intexta.
Localities.

Turin. - - - Cabinet.

Near Waterford.

Chazy.

Middleville.

Near Waterford

\section{MEDINA SANDSTONE.}

\section{PLANTS.}

3821 ARTHROPHYCUS HARLANI.

3832 Arthrophycus -

3842 Scolithus VerTICALIS.

3857 PaLeophycus tortuosts.

3861 Dictuolites beckil(1).

3875 Chдтetes

BRACHIOPODA.

38812 Lingula cuneata.

38922 Atrupa oblata.

39023 A tripa plicata.

39117 Modiolopsis orthonota.

39218 Modiolopsis? primigeniUs.

39319 Pleurotomaria? pervetusta(2). Medina and Lockport. Cabinet.

39420 Plevrotomaria litorea.

39515 Murchisonza? CONOIDEA.

3967 Bucania trilobatus.

3972 Oncoceras grbbosum.

39826 Orthoceras —.

39927 Orthoceras MULTiseptum.

4002 Cytherina Cyirndrica.
Medina, Lockport, etc.Cabinet.

Medina and Lockpoitt.

Monroe county. - - Cabinet. Mouth of Genesee river.Cabinet. Medina. - . Cabinet. Lockport.

Medina, Lockport, etc. Cabinet.

Lockport.

Lockport.

Medina and Lockport.

Medina and Lockport. Cabinet.

Lockport.

Lockport.

Medina.

Lockport.

Lockport.

Lockport and Medina.

Medina. 


\title{
8. CLINTON GROUP.
}

\author{
PLANTS. \\ Localities.
}

4016 Buthotrephis gracilis(1). New-Hartford. - - Cabinet.

4027 Buthotr.

4038 Buthotrephis gracilis, var. crassa. Oneida county, ete.

4049 ButhotREPHIS GRACILIS?

40510 Buthotrephis palmata(1). - . - . - - Cabinet.

40611 Buthotrephis impddica(1). New-Hartford. - - Cabinet.

40712 Buthotrephis ramosa(1). New-Hartford. - Cabinet.

4088 PaLfophycus? striatus. Clinton.

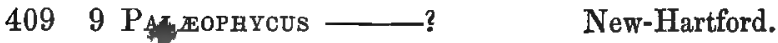

41010 Palarophycus - - ? New-Hartford.

4111 Rusophycus clavatus. New-Hartford.

4122 Rusophycus subangulatus. New-Hartford.

4133 Rusorhycus pudicus. New-Hartford.

4144 Rusophycus bilobatus(4). Near New-Hartford. Cabinet.

4151 IChNophycus tridactylus. New-Hartford.

Tracks of marine animals (three specimens). - - - Cabinet.

CORALS.

4.1615 Graptolithus clintonensis(1). Sodus, Williamson, etc.Cabinet.

41716 Graptolithus venosus.

Below Rochester.

1012 Chetetes lycoperdon.

Sodus, Rochester, etc. Cabinet

4182 Favistella favosidea.

Rochester, etc.

41.91 Caninia bilateralis(1).

Lockport, Rochester, etc.Cabinet.

4201 Cyclolites Rotuloides.

Near Clinton village.

4211 Cannapora JUNCIFormis.

Ontario, Rochester, etc.

4221 Catenipora escharoides(1).

Clinton, Oneida. - Cabinet.

4231 HeLotora fragtLis.

Rochester, Lockport, etc.

4247 Stictopora crassa.

Wayne county, etc.

4258 STICTOPORA RARIPORA.

Rochester.

4261 Phenopora explanata.

Probably at Lockport.

4272 Phenorora constellata.

Wayne county. 
Names.

Lncalities.

4283 Phanopora ensiformis. Flamborough head (Canada West).

4291 Rhinopora verrucosa.

4302 Rhinopora tubULOSA.

4314 Reterora angulata.

4321 FenesteliLa frisca? (1).

4332 fienestella tenuis.

\section{BRACHIOPODA.}

43413 Lingula oblonga(2).

43514 LingtuaA OBLata.

43615 Lingula perevata.

43716 Lingula rameliata.

43817 Lingula acUtirostra.

43921 ORTHS CIRCULUS:

44022 ORthIS ELEGANTUAa? var.

44123 ORTHLS TRINUCLEUS.

44224 ORThIS TENUIDENS.

1469 LEPTIANA SERICEA.

44318 LEPT

44419 LEPT BNA RATENTA.

44520 LePtidena profunda.

44621 Lefterna obscura.

44722 LEPT:ANA ORTHIDIDEA.

44823 LEPTTANA DEPRESSA.

4491 Strophodonta prisca.

4501 Chonates Cornuta.

1721 Sptrifer biforatus, var, LyNX. Rochester, ete.

4512 SPIRIFER

452 3. SPIRTFER RABlatUS.

45324 Atrypa congesta.

45425 Atripa quadicicostata.

45526 Atrupa bidens.

45627 Atrupa NRGLECTA.
Flamborough head.

Sodus, ete.

Sodus and Rochester.

Ontario, Rochester, etc. Cahinet.

Wayne eounty.

Martville, Cayuga. - Cabinet.

Sodus and Wolcott.

Rochester.

Near Clinton village.

Niagasa county.

Sodus.

Wayne county.

New-Hartford.

Rochester and Sodus. Cabinet.

Rochester, ete.

Medina.

Lockport.

Kirkland, Oneida.

Kirkland.

Kirkland, ete.

Kirkland.

Sodus.

Sodus.

Wayne and Niagara counties.

Rochester, Medina, etc.

Lockport.

Lockport.

Niagara county $(1)$. 
Names.

45728 Atrypa equiradiata.

45829 Atrypa emacerata.

45930 Atrypa robusta.

46031 Atrypa Reticularis。

46132 Atripa PIICATULA.

46233 Atrypa HeMISPHERTEA.

46334 Atrypa PLANoconvexa.

46435 A.TRYPA NAVIFORMIS.

46536 Atrupa cyLiNdRICA.

46637 Atripta intermedia.

46738 ATRYY'A 亡-س (cast).

46839 ATRYPA CYLINDRICA? (cast\%

46940 Atripa gibbosa.

4701 Pentamenus oblongus( 1 .

4712 Pentamerus fornicatus.

ACEPHALA.

4726 AVICULA EMACERATa.

4737 Avicula RHOMbOIDEA.

47419 Modiolopsis subalatus.

4756 Tellinomya lata,

4767 TELLINOHYY IMACHERIFORMIS.

4778 Tellinomya cukta.

4784 Orthonota cUtrtí.

4791 PoßIDONIA? alata.

4801 Pyrenomeus cuneatus.

GASTEROPODA.

4811 Crclonema cancellata.

4822 Cíclonema ventricosa.

483 ₹ Cyclonema? obsoleta.

4844 Cyclonema canceldata?

4851 Platyostoma

48616 Murchisonia subulata.
Localities.

Oneida county.

Sodus, Rochester, etc

Liockport:

Sodus:

Niagaira county.

Rochester, etc.

Flamborough head (C. W. )。

Sodus and Rochester.

Lockport:

Iockport.

Kirkland:

Lockport:

Near Clinton village.

Wayne county. - Cabinet.

Lockport.

Kirkland and Wolcott.Oabinet,

New-Hartford and Sodus:

Rochester, Sodus, etc:

Wolcott; Wayne.

Wolcott:

Wolcott.

Wolcott and Rochester.

Rochester:

Wolcott. : - - Cabinet.

Sodus, etc.

Sodus.

Medina and Lockportt:

Lockport.

Kirkland and New-Hartford.

Wolcott, Medina, etc. 
Names.

4878 Bucania stigmiosa.

4889 BUCANIA? BELLA-PUNCTA.

3967 Bucania trilogata.

CEPHALOPODA.

4893 Oncoceras subrectum.

4905 Ormoceras vertebratum.

49128 Orthoceras virgulatum,

49229 ORTHOCERAS ANNULATUM?

49330 Orthoceras abrUttum.

INCERTA SEDES.

4941 CoRnultes flexuosus,

4951 Diścosorus conoideus.
Localities.

Lockport,

Wolcott.

New-Hartford,

Lockport.

Niagara coupty,

Lockport, etc;

Wolcott.

Lockport.

Lockport.

Ontario and Lockport.

UPPER GREY SANDSTONE OF THE CLINTON GROUP.

4961 Myalina mytiliformis.

49720 Modiolopsis ovatus(2).

49821 Modiolopeis subcarinatus(4).

4999 Tellinomya elifiptica.

5003 Pentamerus .ovalis.

44621 LEPTENA OBSCURA?

5012 Platyostoma

50231 Orthoceras ctavatum. 5041 IChTHYODORDLite (fragment).

South of Mohawk village,

S. of Mohawk village. Cabinet.

S. of Mohawk village. Cabinet.

S. of Mohawk village.

New-Hartford.

New-Hartford.

New-Hartford.

South of Mohawk village.

5031 Homalonotus delphinocephalus(4). S, Mohawk village.Cabinet.

\section{NIAGARA GROUP.}

\section{CORALS.}

5057 Streptelasma calicula.

5061 Polydilasma turbinatum.

4191 Caninia bilateralis.

5071 Conophyllum niagarense.

5081 Diplophylum cespitosum.
Lockport, Rochester, etc:

Lockport.

Lockport.

Niagara county:

Lockport. 
Names.

5092 Diplopirllum Caspitosum?

5101 Syringopora? MULTicaults.

5111 Astroceriun VEnUStum.

- 5122 Astrocerium farasiticum.

5133 AstroceriUM PXRIFORME.

5144 Astrocerium constrictum.

5151 Favosites NIAGarensis.

5162 Favosites favosa?

5171 Catenjpora escharoides(1).

5182 Catenipora agglomerata.

5191 Heliolites elegans.

5202 Heliolites spinipora.

5213 Helioutes pyriformis?

5224 Heliolites macrostylus.

5231 Stromatopora CONCENTRICA.

5241 Cladopora seriata.

5252 Cladorora cespitosa.

5263 Cladopora cERvicornis.

5274 Cladopora fibrosa.

5285 Cladopora multipora.

5296 Cladopora MacropHora.

5307 Cladopora reticulata.

5311 Limaria ramdlosa.

5322 Limaria fruticosa?

5333 Liminara iaminata.

5341 Callopora elegantula.

5352 Callopora florida.

5363 Callopora laminata.

5374 Callopora aspera.

5385 Caliopora nimmirormis.

5391 Trematofora tuberculosa.

5402 Trematopora coalescens.

5413 Trematopora tubulosa.
Localities.

Lockport.

Lockport and Barre.

Lockport, Roehester, etc.

Near Lockport.

Rochester, Wolcott, etc.

Lockport, etc.

Niagara falls, etc.

Milwaukie (Wisconsin).

Near Rochester. - Cabinet.

Sweden and Ogden,

Lockport.

Lockport.

Lockport.

Milwaukie (Wisconsin),

Lockport, Rochester, etc.Cabinet.

Lockport, etc. - Cabinet.

Lockport,

Lockport.

Lockport:

Lockport.

Lockport.

Lockport.

Lockport.

Lockport.

Lockport.

Lockport.

Lockport.

Lockport.

Lockport.

Lockport.

Lockport.

Lockport.

Wayne county. 
Names.

5424 Trematopora punctata.

5435 Trematopora ostiolata.

5446 Trematopora solida,

5457 Trematopora striata.

5468 Trematopora granUlifera.

5479 Trematopora aspera.

54810 Trematopora spinulosa.

54911 Trematopora sparsa.

5501 Striatopora flexuosa.

5519 Stictopora pUnctipora.

5521 Diamesopora dichotoma.

5531 Clathropora alcicornis.

5542 Clathropora frondosa.

5555 Retepora diffusa.

5566 Retepora asperato-striata.

5571 HoRnera? DICHOTOMa.

558 3 FenesteLLA ELEGANS.

5594 Fenestella tenuiceps.

5605 Fenestella cribrosa.

5616 Fenestella

5621 Polypora incepta.

5631 Ceramopora imbricata.

5642 Ceramopora incrustans.

5653 Ceramopora foliacea.

5663 Rhinopora tUberculosa.

5671 Lichenalia concentric.A.

5681 Sagenelia Membranacea,

5691 Dictuonema Retiformis,

5702 Dictuonema gracilis.

5711 Inocaulis petumulosa,
Localities

Lockport.

Lockport, Rochester, etc.Cabinet.

Lockport.

IJockport.

Lockport.

Lockport.

Lockport.

Lockport.

Lockport.

Lockport.

Lockport.

Lockport.

Lockport.

Lockport.

Lockport.

Lockport and Rochester.

Lockport, Rochester, etc.

Lockport, Ruchester, etc.

Lockport.

Lockport.

Lockport, Rochester, etc.

Lockport.

Lockport.

Lockport.

Lockport.

Rochester and Lockport.

Rochester and Lockport.

Lockport, Rochester, etc.Cabinet.

Lockport.

Lockport, Rochester, etc. 


\section{CRINOIDEA OF THE CLINTON GROUP.} Names.

Localities.

5721 Closterocrinus elongatus. Locliport.

5732 Griptockintus plumosus. Oak-orchard creek,

5743 Glyptocrinus - N ? - Niagara county:

5751 IсHтHYocrinus clintonensis. Niagara county.

5761 Caryocrinus ornatus. Lockport.

5772 Tentaculites minutus. Rochester.

5783 Tentacuhiteps distans. Flamborough head (Canada West):

Joints of undetermined crinoidea(1). S. Mohawk village.Cabinet,

CRINOTDEA OF 'THE NIAGARA GROUP.

5791 Homocrinus parvus.

5802 Homocrinus cylindricus.

5811 Glyptaster brachiatus.

5821 Thysanocrinus lilimformis.

5832 Thysanocrinus canaliculatus. Lockport.

5843 Thysanocrints actleatus. Lockport,

5854 Thysanocrinus immaturos. Lockport,

5361 Myelodactyliss convolutus. Lockport.

5871 Dendrocrinus LONGIDACTyLOS. Lockport,

5882 Ichthyocrinus Lêtis.

5891 LyRiocrints DaCtylus.

5901 Lecanocrinus macropetaius.

5912 Lecanocrinus ornatus.

5923 Lecanocrinus simplex.

5934 Lecanocrinus caliculus.

5941 Macrostylocrinus ornatus.

5951 Saccocrinus speciosus.

5961 Eucalyptocrinus decorus.

5972 Eucalyptocrinus c

5983 Eucalyptocrinus papulosus.

5991 Stephanocrinus angulatus,

6002 Stephanocrinus GEMmiformis. Lockport.
Lockport.

Lockport.

Lockport.

Lockport,

Lockport.

Lockport.

Lockport.

Lockport.

Lockport, Rochester, ete,

Lockport.

Sweden, Monroe.

Lockport. 
Natries.

6011 Caryocrinus orNatus.

6021 Melocrinites iculptus.

6031 Heterocystites armatus.

6042 Myelodactylus brachiatus.

605 3 Myelodactylus

Plates and columns of undetermined crinoidea.
6061 Callocystjtes jewettil.

6071 Aptocystites elegans.

6081 Hemicystites parasitica.

ASTERIAD $\approx$.

6091 PaLEASTER NIAĞARENSIS.

BRACHIOPODA:

43716 Lingula lamellata.

6108 Orbicula tenuthamellata:

6119 ORBICULA? SQ́UAMIFoRMIs:

61225 ORthis pisulu.

61326 Orthis PYRahlidatis،

44022 Orthis elegantula.

61427 Orthis HYBRIDA.

61528 ORTHAS PUNCTO-STRIAT́A.

61629 Orthis FLABELIULUM, var?

61730 Orthis fasciata.

61824 LeṔt

44823 Lefterna DEPRESSA.

61925 Lept正然a striata.

62026 Leptefna subplana:

6214 SHiRifer bIlobus.

6225 Spirifer sulcatus.

6236 Spirifer crispus.

6247 Spirafer bicostatus.

6258 SPIRIFER NIAGARENSIS.
Localilies.

Lockport, Rochester, etc.Cabinet.

Lockport:

Lockport:

Lockport.

Lockport.
Iockport.

Lockport.

Lockport:

Lockport

Lockport, Sweden, etc.

Lockport.

Rochester, Sweden, etc

Wolcott.

Lockport.

Wolcott, etc.

Wolcott; etc.

Lockport.

Lockporty Sweden, etc.

Rochester and Lockport.

Near Rochester, ete.

Lockport, Rochester ${ }_{i}$ etc.

tockport, etc.

Wolcott and Lockport.

Lewiston, Lockport, ete.

Lockport, Lewiston, etc.

Oneida county.

Wolcott, Rochester, etc. 
NEW-YORK STATE COLLECTION.

Names.

4523 Spirifer fiadiatús.

6269 SpIrifer pyramidalis.

62741 Atrypa nitida.

62842 AtRypa nitida, var, oblata.

62943 Atrypa

63044 Atrypa crassirostra.

46031 Atryfa retidUlaris.

63145 Atrupa rugosa.

63246 Atrupa nodostriata.

63347 Atrypa camura.

45627 Atrypa nEGLecta.

63448 Atrypa interplicata.

63549 Atrypa bidentata.

63650 Atripa cuneata.

63751 ATRYPA

63852 Atripa disparilis.

63953 AtrYPA BREVIROSTRIS.

64054 Atrypa obtusiplicata.

64155 Atrypa plicatella?

64256 ATRXPA APRINIS.

64357 Atrupa corallifera.

\section{ACEPHALA.}

4726 Avicuta emacerata.

6448 Avicula undata.

6459 Avicula subplana.

64610 Avicula? orbiculata.

6472 Posidonomya? rhomboidea.

64822 Modiolopsis? undulostriata.

47419 Modiolopsis subalatus?

4784 ORTHONOTA CURTA?
Localities.

Lockport, Lewiston, etc.

Near Lewiston.

Iockport, etc.

Loekport and Sweden.

Isockport.

Lockport.

Jockport, ete.

Lockport.

Lockport.

Lockport.

Wolcott, Lockport, etc.

Lockport.

Lockport.

Lockport, etc.

Irockport.

Wolcott.

Irockport.

Lockport.

Wolcott.

Iockport.

Lockport and Rochester.

Lockport, Rochester, etc.Cabinct.

Rochester.

Lockport.

Rochester.

Lockport.

Lockport.

Wolcott.

Wolcott. 


\section{GASTEROPODA. Names.}

6492 Platyostoma niagarensis.

6503 Ptatyostoma hemispherica.

651 1. Acroculia NIAgarenisis.

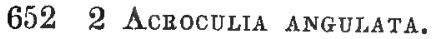

CEPHALOPODA.

6531 Gomphoceras? - -

6548 Cyrtoceras? cancellatum.

65532 Orthoceras - - .

65633 Orthoceras imbricatum?

65734 ORThoceras VIRgatUM?

65835 Orthoceras CANCELlatum.

65936 Orthpgeras _- ?

66037 Orthockras UNDULATUM.

6615 Conularia niagarensis.

6626 CoNuLARIA LONGa.
Localities.

Lockport, Rochester, ete.

Rochester.

Lockport.

Lockport.

Rochester and Niagara falls. Rochester and Niagara falls. Rochester.

Lockport and Rochester.

Rochester.

Rochester and Lockport.

Rochester.

Luckport, Rochester, etc.

Lockport, Rochester, etc.

Locliport.

CRUSTACEA OF THE CLINTON AND NIAGARA GROUPS.

6631 Cybele punctata.

6645 Caltmene clintoni.

2982 Calymene bldmenbachil, var?? senaria.

6653 ACIDAgYIS - -

6663 Phacops trisulcatus.

6675 Ceraurus insignis.

6681 Beyrichia lata.

6691 Bumastis BarriENSIS.

6704 Phacops limulurus(4).

6717 Calymene blumenbachil, var. niagarensis(1). Lockport,

5031 Homalonotus deiphinocephalus (2), Lockport, ete. Cabinet.

6721 Lichas boltoni(2).

673 I Bronteus? niagarensis.

6741 Arges fhlyctanodes.

6751 Proetus coryecets(1);
Rochester, etc. Cabinet.

\section{Rochester.}

New-Hartford, etc. Cabinet. Lockport, etc. - - Cabinet. Lockport. - - Cabinet. Lockport, Rochester, etc.Cabinet. Niagara river. Near Albion, Orleans.

Lockport. : - - Cabinet. 
Names.

6762 Proetus? stokesiI.

6772 Bevrtchia symmetrica.

6782 Cytherina spinosa.

1 ONCHUS DEWEII.
Localities.

Lockport.

Lockport. - - Cabinet.

Lockport.

Lockport and Rochester.

\section{CORALLINE LIMESTONE.}

\section{CORALS.}

679 \& Diplophyllum coralliferum(3). Schoharie. - - Cabinet. 6801 Columaria inequalis(1). Schoharie. - - Cabinet. 5151 Favosites nialararensis(2). Schoharie. - - Cabinet. 6812 Stromatopora constellata(1). Schoharie. - - Cabinet. 5231 Stromatopora concentrica(1). Schoharie. - - Cabinet. 5171 Catentpora escharoides(1). Schoharie. - Cabinet. BRACHIOFODA.

68231 Orthis interstriata.

68327 LEPT AENA

68428 Laptana bipartita(2).

6852 Strophodonta textilis(1).

68610 Spirifer (2).

6236 Spirifer crispus.

68758 Atrtpa nucleolata(3).

68859 Atrupa lameliata(1).

68960 Atrypa

\section{ACEPHALA.}

69010 Telimomya? equilatera(2).

69111 Avicula?

69212 Avicula subrecta.

69313 Avicula securiformis(3).

69414 Avicula Lim EForials(2).

GASTEROPODA.

6954 Pratyostoma (1). 69621 Pleurotomarta subdepressa(1). Schoharie. - - Cabinet. 69717 Murchisonia? obTusa(1).
Schoharie. - - Cabineț.

Schoharie.

Schoharie. - - Cabinet.

Schoharie. - - Cabinet.

Schobarie. - - Cabinet.

Schoharie, - - Cabinet.

Schoharie.

Schoharie. - - Cabinet.

Schoharie. - - Cabinet.

Schoharie. - Cabinet.

Schoharie. - - Cabinet.

Schoharie. - - Oabinet.

Schoharie.

Schoharie. - - Cabinet.

Schoharie. - - Cabinet.

Schoharie. - - Cabinet. 
Names

69818 Murchisonia? terebralis(1).

69910 Bucania (3).

7005 Bellerophon auriculatúg(2).

\section{CEPHALOPODA.}

7011 Trochoceras gebhardil(2).

7022 Trochoceras turbinata(1).

7034 Oncoceras expansum(1). Orthoceras (4).
Localities.

Schoharie." - - Cabinet.

Schoharie. - - Cabinet.

Schoharie. - - Cabinet.

Schoharie. - - Cabinet.

Schoharie. - - Cabinet.

Schoharie. - - Cabinet.

Schoharie. - - Cabinet.

\section{CRUSTACEA.}

7048 Calymene camerata (5 fragments). Schoharie - - Cabinet.

7053 Cytherina ALta?

Paragmbceras?(2).
Schoharie.

Schoharie. -

\section{ONONDAGA-SALT GROUP.}

7064 Pentamerus occidentalis.

7071 Megalomus canadensis.

70819 Murchisonia eivittata.

70920 MÚtehisonia mongispira.

71021 Murchisonia bovdll.

71122 Murchisonia toganil.

71223 Murchisonia miacrospira.

71324 Murchisonia trüritiformis.

7142 Subueites ventricosa.

7155 Cyclonemid sulcata.

71622 Pletjrotomaria bispiralis.

71723 Plentotomaria?

71824 Pleurotomaria solarioldes.

71925 Plenrotomakia perlata.

72011 Bucania angustata.

7219 Cyrtoceras arcticameratum.

7229 Calymene

7234 Tentaculites niagarensis.
Galt (Canada West).

Galt.

Galt.

Galt.

Wayne county.

Galt (Canada West).

Galt.

Galt.

Galt.

Newark, Wayne.

Galt (Canada West).

Galt.

Galt.

Galt.

Galt.

Galt.

Galt. 
The Colemction of Fossils made by John Gebhard junior has been purchased by the State, and is now in the State Geological Rooms at Albany, but, for want of room, is not yet arranged. The following is a synopsis of its contents, with the exception of the fossils from the Coralline limestone (mentioned on pages 28 \& 29), which have been placed in the eases:

Names of Formations.

Tumber of Specimens.

Calciflerous sandrock ................. 2

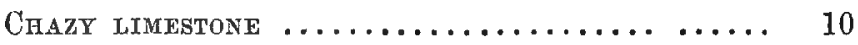

Birdsete Limestone ..................... 22

Trenton Limestone.................... 92

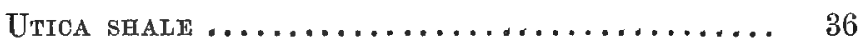

Medina sandstone..................... 9

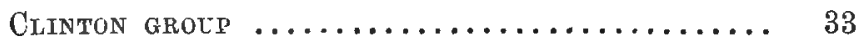

NIAGARA GROUP, Lockport................ 132

Hudson-RIVER group................... 5

Coralline limestone, $\quad$ Sehoharie......... 79

Tentacultte limestone, $\quad$ " $\quad \ldots \ldots \ldots .630$

Pentamerus galeatus limestone, " $\ldots \ldots \ldots .672$

Catskill shaly limegtone, " "

Scotelda limestone, $\quad$ " $\quad \ldots \ldots \ldots .6 .6$

Upper Pentamerus limestone, "

Oriskany sandstone, $\quad$ " $\ldots \ldots \ldots .657$

ScHOHARIE GRIT, " "

ONONDAGA LIMESTONE, " "

CoRniferots LIMESTONE;

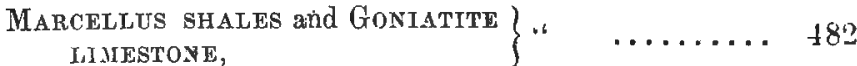

HAMILTON GROUP (Lower part), “ $\ldots \ldots \ldots . .1423$

Hamilton aroup (Upper part), including Chemung and
uppermost rocks of Schoharie, 1313

Plaster casts of fossills (mostly trilobites) ....... 131

Frsh scales (two species), Old Red, Pennsylvania.... 2

Coal plantrs from Pennsylvania .............. 37

Co.al Plants from Ohio ................... 3

Fisil Remains from Durham, Connecticut.......... 7

Bourstone formation of Georgia ............. 11

Tertiary, Macon, Gcorgia .................. 2

Tertiary, Banks of the Potomac .............. 2

Cretaceous formation of New-Jersey .......... 5

Tertiary formations of Italy $\ldots \ldots \ldots \ldots \ldots \ldots .46$

DuDley roors, Fngland $\ldots \ldots \ldots \ldots \ldots \ldots \ldots \ldots, 48$ 
The preceding specimens are packed in forty-one boxes properly labelled, and are now in the fireproof basement of the State Geological Rooms.

In addition to the above, there are, in drawers in the geological rooms, the following numbers of unique specimens, viz:

Chazy limestone, 2; Trenton, 10 ; Hudson-river, 9 ; Niagara, 4; Pentamerus galeatus, 5; Delthyris shaly, 41; Oriskany sandstone, 6; Schoharie grit, 10 ; Marcellus shale, 2; Hamilton group, 9. Total, 98.

Fish nemains : Schoharie grit, 9 ; Onondaga, 2 ; Marcellus shale, 11 ; Hamilton, 5 ....................... .

Specimens figured for the third volume of the Palæontology, and placed in the cases $\ldots \ldots \ldots \ldots \ldots \ldots \ldots \ldots \ldots \ldots .103$

Which, by including the preceding list, makes a total of ....... 13292

In addition to these, there have been placed in the cases over six hundred (600) specimens of fossils from the following formations, viz : Tentaculite limestone, Pentamerus galeatus limestone, Delthyris shaly limestone, Upper Pentamerus limestone, Oriskany sandstone, Schoharie grit, Onondaga limestone, Corniferous limestene, Marcellus shale and Hamilton group.

The collections made by Prof, HaI. since the commencernent of the work on Palæontology, with the exception of those enumerated in the Catalogue, are now lying in the basement story of the Geological Buildings : they consist chiefly of the following: Ninety-six drawers in case, flled with specimeus from differcnt rocks and groups.

Nine similar drawers filled witl specimens, not in case.

Three drawers with specimens.

Forty-five large drawers in close cases, flled with specimens of the fossils of the lower limestones, Helderberg himestones, Hamilton and Chemung groups. Many of these are very good and perfect specimens of fossils, intended at the time of collection to illustrate future volumes of the Palæontology.

Three boxes of the Hamilton group and Helderberg linestones.

Forty-nine boxes and two kegs of specinens mainly froin the rocks and groups above the Niagara. These have been nearly all examined, and the better specimens separated from the others, and their character indicated by labels on the otitside of the boxes.

Fourteen boxes of specimens of fossils and minerals, mainly of the Niagara group.

There is also a large number of slabs containing fossils, many of which are rare and valuable. 

然istocicat and Antignaxian collection. 



\section{HISTORICAL AND ANTIQUARIAN COLLECTION.}

\section{DONATIONS.}

FROM LEWIS H. MORGAN, OF ROCHESTER.

1. Ga-né-ga-tah (STONe Mortar), used by the Senecas fur pounding corn, pulverizing roots, etc. : sandstone. Found near Allen's hill, Ontario county, May 9, 1843.

2. Stone pestle, found at Jack's reef, Onondaga county.

3. StOne PESTle, found in the town of Middlebury, Wyoming county.

4. Stone chised, from Groveland, Livingston county.

5. Stone chisel. Fort Hill, town of Seneca, Ontario county.

6. Stone chisel. Town of Coventry, Genesee county.

7. Stone chisel. Ontario county.

8. Stone chiser, used for chipping coal. Iiort Hill, town of Seneca, Ontario county.

9. Stone chisel, from Auburn, Cayuga county.

10. Stone chisec, used by the Iroquois in felling trees : fire was applied near the root of the tree, and the chisel was used to cut out the coal ; after which, fire was again applied. The chisel and fire were also used to hollow out wooden ressels. From Tonawanda, Genesee county.

11. Stone chisel. From the town of Coventry, Genesce county.

12. Stone caisel. From Mendon, Monroe"county.

13. Stone chisel. From Allen's bill, Ontario county.

14. Stone chisel. From Mendon, Monroe county.

15. Stone chisel. From Coventry, Genesee county.

16. Stone gouge. Found near Leroy, Genesee county. 
17. Spear-head (chert). Found at Fort Hill; Seneca, Ontario county.

18. Arrowhead. Town of Seneca, Ontario county.

19. Arrowhead. Town of Chili, Monroe county.

20. Arrowhead. Fort Hill, Ontario county.

21. Indian knife (chert), used for skinning decr. Found near Avon at the site of Littlebeard's town, Livingston county.

22. Tomanawr, found by the skeleton of an indian, near Lima, Livingston county.

28. Tomahawk, found by the skeleton of an indian at Mendon, Monroe county.

24. Tomahawk. Town of Lima, Iivingston county.

25. Tomahawk. Town of Ledyard, Cayuga county.

26. Brass KETtLe, taken from an indian grave at the site of the old indian village of Gá-nun-da-sa-ga, near Geneva, 1840.

27. Pakt of a GUN Lock and Rifle barrel found by the skeleton of an indian, in the town of Mendon, Monroe county.

28. HEAD of a $G a-n e-a h$ or WAR CLUB, fastened into the head of a club by a thong or withe. Ledyard, Cayuga county.

29. Yuh-tah-gun-lue-a-tah Geh-weh, or MocCAsIN NEEDLE (bone of the deer). Used by the "mound-builders," and also by the Iroquois. Found at Fort Hill, near Leroy, Genesee county.

30. Stone tirbe (of variegated limestone). Town of Springport, Cayuga county. A relic of the Mound-builders, and not of the Iroquois. Similar tubes, some of which are fifteen inches in length, are found in the Ohio mounds.

31. Stone amulet. Tonawanda, Genesee county.

32. Indian NECKLACE of TEETh, found near the skeleton of an indian. Genesee valley, near Avon, Livingston county.

33. A-so-gwa-ta, or ClaY pIPE, from Aurora, Cayuga county،

34. Front Part of the Bowl of a PIPE (similar to those found in the Ohio mounds). Lima, Livingston county.

35. Fragment of Ga-jeh, or Earthen basin. Fort Hill, near Leroy, Genesee county; with a tooth found in the same place.

36. Potrery, affected by water. Fort Hill.

37. Gus-to-weh : Head-dress. Seneca - Iroquois.

38. Ga-neah, or WAR cLub : a species used in the war dance. SenecaIroquois. 
39. Indian calumet. From the West (imperfect).

40. Leanen cross. Found near the Tonawanda Council House, Genesee county, Oetober, 1845.

41. Fragment of the tombstone of Redjacket, found upon his grave, four miles from Buffalo. The stone is mutilated by travellers.

42. Fragment (slag?) taken from the fireplace of the old picket enclosure of Kon-non-da-sa-sa, at the foot of the Geneseo lake, in 1845. This picket was destroyed by General Sullivan.

43. Sтuв of the palisade at the gate or opening of the above picket enclosure. Found November 21, 1845.

44. Fragment of an Indian pipe. Monroe county.

45. A-se-qua-tah, or Clay pIPE, taken from a Seneca burial place near Lima, Livingston county, 1848.

46 \& 47. Arrowheads. Mendon, Monroe county.

48. Arrowhead of copper. Bend of the Honeoye creek, Monroe county.

49. Part of a gUn-barkel, from an indian burying-ground, Ball farm,

Monroe county.

50. Stone shdld-cracker. From Aurora, Cayuga county. This is the vulgar name : it was fastened to the head of a club, and thus made a formidable weapon.

51. Necrlace bead. From Scipio, Cayuga county.

52. Unfinished arrowhead. From Cayuga county.

53. White chert arrowhend. From Ledyard, Cayuga county.

54. Fragment of the Bowl of an Ah-so-quä-tä, or PIPE. From Scipio, Cayuga county.

55. Six arrowheads, or Gä-nuh-yä. From Ledyard and Scipio, Cayuga county.

56. Fragment of a white chert arrowhead.

57. Two twist arrowheads. From Ontario county.

58. Fragment of a Ga-jih, or earthen basin. From Cayuga county.

59. Gä-ne-gä-tä, Seneca mortar, for pounding corn.

60. Gä-nih-gä-dä, Pounder (same name as mortar). Two specimens.

61. Gü-ne-ah, Ball ват. Two specimens.

62. Wë-a=no, Indian bow. Two specimens.

63. Gä̈no, Frathered arrow. Six specimens.

64. Gä-ppä-sü, Snow snare. Two specimens: 
65. Bark tray or platter.

66. Splint baskis. Two rarieties.

67. A quantity of White cons. The New-York Indians cultivate this variety of corn principally, which is known, I believe, as the Tuscarora. They put it up and preserve it in bunches.

\section{FROM SAMUEL G. EDDY, OF S'TILLWATER.}

68. Card of indan arrowheads(17), found in immediate vicinity of the battle ground at Bemis's Heights, Suratoga county. Arrowhearls of similar materials and construction are to be found, after the spring rains, on all the plowed lands between Stillwater village and Wilber's basin, a distance equal to fire miles.

69. Cannon Balls(2), found on the battle ground at Bemis's heights.

70. CARD of MiLitaRy ButTons(5), with the roman numerals XX distinetly visible on the faces. These buttons were worn by a soldier or soldiers of the 20th regiment of Hamilton's brigade in Burgoyne's army. The 9 th, $20 \mathrm{th}, 21 \mathrm{st}$, and $62 \mathrm{~d}$ regiments were that portion of Burgoyne's army which was engaged in the bloody conflict at "Freeman's Cottage," Bemis's Heights, on the 19th day of September 1777. These buttons, together with human bones, a large pocket knife, belt buckle, pewter spoon, and a stick of healing salve, were plowed up in the month of October 1849, within the grounds enclosed by the British intrenchments.

71. Lead balls and Iron grape-shot (13 in all), found on the Freeman farm in the ycar 1848.

72. A Spanish silver corn (pistareen), dated 1721. This coin, together with two others of the same date and denomination, three spanish milled quarter-dollars, twelve guineas and two half joes (in all about eighty dollars), were plowed up by Mr. Ebenezer Leggett, in the fall of 1849, within the British intrenchments, and near the celebrated Freeman Cottage.

73. P1EcE of the PIAANK on which Gen. Frazer DIED. This gallant officer was mortally wounded on the seventh of October 1777, about two miles west of the Hudson river, by a rifleman of Colonel Morgan's company. He was brought from the field of battle and taken to the "Smith House" (then used as a British hospital), which was situated on the Whitehall furppilue some six miles 
north of the present village of Stillwater, and expired about eight o'clock A. M. the following day. By his own request, he was buried in the great redoubt on the hill, at six o'clock in the evening of the satme day on which he expired. The Smith house was taken down in the year 1845, and the plank on which Gen. Frazer died, and of which the piece presented to the State Cabinet is a portion, was preserved by the proprietor for the antiquarians of his country.

74. Piece of a soldien's blanket, dug up, with human bones, near the camp of Lord Balcarras (who was one of the commanding officers in Burgoyne's army), at Bemis's heights, seventy-one ycars after the battle of October 7, 1777 .

75. A вомв sHell. This was one of the trophies taken at Bemis's heights in October 1777. The following spring, several batteaux were loaded with shells, cannon balls, etc. for shipment to Albany, one of which was sunk at Stillwater village, a short distance above the falls, in the Hudson river. During the season of low water in the summer of 1848 , many of these shells and balls were dug from the bed of the river, one of which is the one here presented to the State Collection.

\section{FROM ASA FITCH, OF SALEM.}

$76 \&$ 77. Sword and $\mathrm{B}_{\mathrm{A}}$ Yonet, which belonged to Burgoyne's army.

Thomas Whiteside, one of the Cambridge (Washington county) militiamen, in service at Saratoga at the time of Burgoyne's surrender, on his return home, brought the above articles : they have been preserved in his family ever since, and were given to the State Collection by his son Thomas C. Whiteside.

78. Indian axe, found at Fort Miller. From John Pattison.

79. Part of an Indian spearhead (made of subhyaline quartz), tound at Fort Miller. From John Pattison.

$80 \& 81$. Indian spearheads. Salem.

$82 \& 83$. Indian arrowheads. Salem.

84. Retics of the Battre at Wallomsac (N. Y.), commonly called "Bennington Battle." Within the fortification thrown up by Colonel Baum, a single oak tree was left standing. The PIECE of wOOD presented to the State Capinet, is from the decaying stump of 
that tree. Sunk in it is a box containing a BULLET, found about forty rods north from the tree, in the direction whence General Stark, it is said, made his attack. The bullet is from N. Burnet, esquire, proprietor of the grounds, who picked it up when plowing about ten years ago (1838). Vestiges of this battle, formerly found in abundance, are now exceedingly rare; and all traces of the breastworks, etc. are entirely obliterated.

\section{FROM WILLIAM J. M'ALPINE, OF ALBANY.}

85. A Plaster model of the United States Dry Docir at Brooklyn.

86. Fourteen specimens of GRANITE, viz : six from Staten Island quarry, New-York; six from Quincy quarry, Massachusetts; and two from Bluehill quurry, Maine; being samples of the granite used in the construetion of the dry dock.

87. A gLaSs tube, hermetically sealed, containing specimens of the various sorts through which the excavations were made, stratigraphically arranged; with figures on the tube, indicating the aggregate depth of the excavations, and the proportional thickness of the different strata of earth excavated.

88. A vial containing EARTH excavated at the depth of $68 \frac{1}{2}$ feet.

\section{FROM JOHN GEBHARD, OF SCHOHARIE.}

89. Egyptian inage, ten inches in length, made of the native sycamore wood, in the form of a mummy case ; painted, and covered with hieroglyphics. Inages of this description are frequently found in mummy cases, and appear to be a representation on a small scale of the mummy itself. It is probable that they were used as a kind of household gods by the ancient Egyptians, and in that character interred with the possessor on his decease.

90. Etruscan figure, representing Pallas. This figure is made of baked clay, and placed upon a marble pedestal ; height eight inches.

91. Bronze bust of the Princess Lucille. Height three inches above the pedestal.

92. Bronze figure of a consul in his toga. Height five inches above the pedestal, 
93. Roman Figure in BRonze. Height four inches.

94. Bronze figure of a cow. Height two inches above the pedestal.

95. Two bronze figures of soothsayers or oracles. Height four inches above the pedestals.

96. Bronze figure of the Egyptian Vents. Height four inches above the pedestal.

97. Three varieties of funeral Lamps. These lamps are made of baked clay, and are of ancient egyptian manufacture.

\section{FROM CHESTER C. MOORE, OF ALBANY.}

98. A Medicine Pouch, manfactured of deerskin, and beautifully ornamented with porcupine quills : made by the Blackfeet Indians of Oregon.

99. A string of stone bEADs, obtained from the Blackfeet Indians of Oregon, and supposed to have been obtained by them from the Russian traders.

100. A flint arrowhead, found in the Blackfeet country near the Rocky Mountains, where a battle had been fought between the Blackfeet and Crow tribes of Indians.

FROM CHARLES MARTIN; OF THE U. S. NAVY.

101. A MACE or WAND, made from the feathers of the macaw or toucan : carried in the hand by the chiefs of the Cannibal Indians of the Amazon river, eighty or ninety miles above Para a city of Brazil.

102. A TUBE or sHEATH made of woven strips of bark or reed, capable of enlargement and contraction : used for the purpose of expressing the liquid from the arrow root, in its preparation for use. From the Amazon river, 80 or 90 miles above Para, a city of Brazil.

103. Two EARTHEN PIPEs, of different style, with a wooden oruamental stem. From the Amazon river above Para.

104. Gound. From the city of Isabel, Guatemala, painted in imitation of the ruins in that country. 
FROM MRS. M. ELIZABETH BALDWIN, OF SARATOGA.

105. A BELT interwoven wich BEADs, and a GAME-BAG. These articles were taken from a vault in Peru, South America, in the year 1830 : they had been deposited with the remains of an indian chief a hundred years previous.

106. A Bill of the continental ctjrancy for sixty dollars, issued according to a resolution passed by Congress at Philadelphia, September 26, 1778.

107. A Bill of the virginia curbency for seven hundred and fifty dollars, issued according to an act of the Assembly, passed March 1, 1781.

FROM THEODORE TEED, OF CORTLANDTOWN.

108. A large stone axe.

109. A STONE CHISEL, made of greenstono.

110. An unfinished chert arrowhead.

111. A stone PESTLE, made of sandstone.

112. A fragment of an INDIAN EOWL, made of steatite (soapstone).

All these articles were found in Cortlandtown, Westchester county.

FROM REV. MILES BRONSON, OF ASSAM IN INDIA.

113. A Silver coin (one rupee), value 48 cents : issued by the East India Company, 1840.

114. A Copper coln (one quarter anna), value 1-48th of a rupee : issued by the East India Company.

115. A Native East-Indian copper colN.

\section{FROM RICHARD H. PE.ISE, OF ALBANY}

116. Gü-0-wo-gus-nuh (bark canoe) : a model.

117. Gä-gü-we-sä (paddle) : four specimens.

118. Ya-ye-gwä-dä-quä (segar case).

119. Ya-yud-dos-ho-quä (card receiver) : two specimens.

120. Gä-gwih-sak-tah Yä-dä-gwah (watch case). 
FROM REV. JOHN N. CAMPBELL, OF ALBANY.

121. A Cup, turned from one of the red cedar gate-posts of Fort William Henry. The posts were placed in the ground in the year 1755, and removed in 1837, and are now in the State Cabinet.

122. A Bead basket, made and presented by Peter Lepage junior, one of the pupils belonging to the New-York Institute for the Blind.

123. A specimen of "Рот Rock," from Hurlgate channel near New-York, procured and forwarded by M. Maillefert, the engineer employed in removing the obstructions to navigation in Hurlgate.

124. A piece of Copper sheeting, taken from Way's reef in Hurlgate channel. Large quantities of this were obtained while removing the reefs in Hurlgate, which had been stripped from vessels that had struck against them at different times.

FROM REV. DUNCAN KENNEDY, OF ALBANY.

125. Two Pequod stone hatchets, differing in form and material of construction. From New-England.

126. Stone axe and a Stone chisel. From Westmoreland, Oneida county.

\section{FROM EDWIN CROSWELL, OF ALBANY.}

127. Copper mexican bomb-shell, taken from the battle ground of Buena Vista by Lieut. Easterly, U. S. Army.

128. CoPfER MEXICAN GRAPE-SHnT, taken from the battle ground of Buena Vista, by Lieut. Easterly.

129. Cannon ball, found in excavating in front of the site of the ancient Fort Orange, Albany.

\section{FROM JAMES MEADS, OF ALBANY.}

130. A Copper mexican Grape-shot, from the battlefield of Buena Vista. 131. A Mass of sparables, cemented together by heat : from the great fire in the eity of Albany on the 17th day of August, 1848.

132. A Mass of TACKS, cemented together by heat: from the great fire in the city of Albany on the seventeenth day of August, 1848. 


\section{FROM VARIOUS DONORS.}

133. A MaP of the town of North-Salem, on rollers : executed by the Donor JoHN F. JenKINS.

134. INDIAN PIPE, found four miles south of the village of Canandaigua. William Case.

135. Remains of the Gate-posts of Fort William Henry, at the head of Lake George. The fort was erected in 1755 , and taken and destroyed by Montcalm in 1756. The posts (of cedar) were dug up in 1837, by the direction of William Caldwell the owner of the ground, and were presented to the New-York State Cabinet by JoHN M'Gillis of St. Johns in Canada East.

136. A Piece of marble (from Maryland), of the corner stone of the Washington National Monument, laid July 4, 1848. Presented by the Board of Managers of the Washington Monument to the State of New-York.

137. Indian ARROWhead (hornstone), found at Lake George.

T. ROMEYn Beck.

138. Stone sKinning-Chisei, found in Lysander, Oswego county.

E. A. BaLdwin.

139. INDIAN ARROWHEAD, found in Watervliet, Albany county.

A. C. Hascy.

140. Stone pestre, used in pounding maize : found at the head of Cayuga lake, Tompkins county, by Henry Hungerford.

David Emery.

141. A Copy of an "Inquiry into the Origin of the Antiquities of America, by John Delafield junior; with an Appendix by James Lakey." 4to. Cincinnati, 1839. John Delafield.

142. Specimens of Sheli concretions, forming the surface rock on which the city of St. Augustine in Florida is built.

\section{Christopier Morgan.}

143. A Plate of mica, turned up by the plow seteral years since, in the town of Brookhaven in the county of Suffolk, at a depth of about two feet below the surface. On one side of this plate are several engravings of geometrical and other figures.

Nathanied Miller. 
144. A part of the stem of the Agave americana (century plant), which

flowered some years since in the greenhouse of

$$
\text { Stephen Van Rensselaer. }
$$

145. Two pieces of wood from the HULL of Commodore Perry's flag-ship, the Lawrence : obtained at Erie, Pennsylvania.

Franklin B. Hodgh.

146. Copper PICKaẊe, found on the farm (in Ogdensburgh, St. Lawrence county) of

Henry Van Renssetaer.

147. Stone chisel : from Danube, Herkimer county. Asa WiLcox.

148. Stone chisel, made of greenstone : from Martinsburgh, Lewis county. L. R. Hovgr.

149. LaRGe SPEARHEad, made of chert : from Martinsburgh.

L. R. Hovgh.

150. Fragments of ancient pottery : from Martinsburgh, Lewis county. S. A. Dewey.

151. Four chert arrowheads : from Martinsburgh. S. A. Dewex.

152. STONE PESTLE, used in pounding maize : found in the town of Niskayuna, Schenectady county. Peter B. Noxon.

153. A S S T TNe AXE, made of chlorite : plowed up in the ninth ward of the city of Albany. Peter B. Noxon.

154. Two cherT and one White quartz arrowheads : found in the town of Guilderland, Albany county. Thaddeus Cheesebro. .

155. A chert arrowhead : found in digging a cellar on the shore of Otsego lake.

S. W. Rоoт.

156. Indian ARrow head : found in Croton, Westchester county.

Theodore Teed.

157. Three fragments of Indian pottery : found in Croton. T. Teed.

158. A very singular specimen of INDIAN WORKMANSHIP, made of novaculite : found in the town of Hartford, Washington county.

Henry B. Northrup.

159. TACKs, cemented together : from the great fire in the city of Albany, on the seventeenth day of August, 1848. Lemued Steele.

160. A specimen of Cocoanut-Wood (turned): from the Island of St. Lucia, West Indies.

Robert Reid.

161. An Antique lookinglass, set in a frame of sandal wood.

Miss Caroline Lovetet. 
162. A Silver coln (fuang), $7 \frac{1}{2}$, cents, from Siam in India.

Rev. A. Hemenw ay.

163. A Silter coin, date 1652 : on one side "New-England," on the reverse "Massachusetts;" commonly called Pine-tree shilling. Hiram H. Cooper.

164. Eleven Chert arrowheads, found in Stugvesant, Columbia county. Aaron Van Alen.

165. A Cannon bali, found near the junction of the Battenkill with the Hudson river, on the farm where Burgoyne erected a breastworls, etc. during the revolutionary war.

Mrs. S. M. Lanstng Merchant.

166. Several Chert arrowheads; also fragments of Human bones and INDIAN POTTERY, obtained from an ancient indian buryingground on the banks of Connecticut river. A. MARKs.

167. An Indian chert arrowhead (large) : found in Bethlehem, Albany county. L. M`Mulden Selkirk.

168. A fragment of Indian pottery : found on the banks of the Hudson river, six miles above the High falls. This fragment, which is two inches long and one and a half wide, represents the head and about one half of the shell of a tortoise : it appears to have been a portion of a pipe.

Ebenezer Emmons.

169. A specimen of Cloth made of the BARK of the BREADFruit TREe, by - the natives of one of the islands of the Pacific ocean.

Ferdinand Weil.

170. A piece of one of the OAK TREES cut down in the town of Argyle, Washington county, in the year 1797, for the purpose of constructing a navy, under the administration of John Adams.

There were at that time two gigantic oaks which grew near together, that were cut for the abovenamed purpose. One of them was hewed, and afterwards drawn by fifteen yoke of oxen, with the forward end elevated on a huge wood-sled. When they had got out of the woods, a "stump speech" was delivered by one of the party from the forward end of the stick, as a stage. After passing the bottle around, according to the custom of the times, they moved on with shouts of overflowing patriotism that almost seemed to inspire even the dull ox with more than his ordinary ambition, and thus delivered the stick at a place then called $\mathrm{Du}$ mont's Ferry, a short distance below Fort Miller.

The other trec, of which the picce presented to the State Cabinet is a part, was cut and scored on two sides, and partly hewed; but 
owing to some small defect, it was abandoned. About twelve years subsequent to that time, it was split into rails, and laid up into fence. Only a few of the rails now remain, and it was with some trouble that a few pieces were obtained which bear the marks of the axes of those who scored and partly hewed the stick; one of which is seen on the piece presented.

James H. Sill.

171. Twio Indian arrowheads, made of greasy quartz; one ditto, made of yellow jasper : from Suffolk county. $\quad$ E. T. Sмiтн.

172. A Stone axe, eleven inches in length. This relic was found several feet below the surface, in digging a well at Poughkeepsie, Dutchess county. A. Heyer Brown.

173. A Military cap plate of the Revolution. Destgn : The American Eagle with extended wings, surmounted by thirteen stars. Мотто : "Unity is Strength," 1776.

Mrs. Mercy Valentine.

174. An Ancient millstone, plowed up in the town of Conesville, Schoharie county, in the summer of 1850 . The stone is graywacke, twenty inches in diameter and two inches thick, and was used in the early settlement of the country in a hand-mill.

\section{Abramali J. Warner.}

175. A Camp knife, dug up by James Stewart, esquire, of Guy Park in the town of Amsterdam, Montgomery county, formerly the residence of Sir Guy Johnson of tory memory in the days of the revolution, and is a relic of those times.

M. S. Goodale.

176. $\Lambda$ Chisel and a Deerskin-Dresser, made of greenstone : found in Chcrryvalley, Ashtabula county, Ohio. Theopore Mills.

177. A Stone axe and a White chert arrowhead : found in Cherryvalley (Ohio).

Theodore Milis.

178. A Stone chisel, and also two regularly rounded pebbles : found in the town of Bethlchem, Albany county. Peter G. Bradt.

179. An Iron KEY, taken from the Halls of Montezuma, Mexico, on the twelfth day of June 1848, and which originally belonged to the palace garden gate.

W. A. Нотснкiss.

180. A Clay drinking cup, in the form of a duck, from the Sandwich islands; also a HEADDRESS made of seashells.

Benjamin Knower. 
181. A part of the Shot-Racr of the British Frigate Hussar, with four CANNON BALLS; also a NUSKET, and a BAXONET and SCABBARD.

The Frigate Hussar was lost December 15th, 1780, off Stoney island on Westchester county shore, having struck Pot rock in Hurlgate. She was loaded with troops, ammunition, and supplies for the British army in this country. The troops and crew had barely time to reach the shore before she sunk, with seventy American prisoners, who were in irons and could not be rescued. These relics were obtained by Messrs. Pratt \& Howe, in the month of August 1851, by the aid of Taylor's submarine armour, after having been buried over seventy years. The wreck lies seventy-six feet deep at high water.

HENRY B. TODD.

182. Four MuskEt-Balis and two Buckshot, from the battlefield of Lundy's Lane, Canada West. James̃ A. Hurst.

183. An Iron key and a Brass belt-slide, dug up at Fort Ticonderoga in the year 1849, by Wildiam ThorN.

184. An Iron WORMER, used for drawing charges from muskets; found on the grounds near Fort William-Henry. Hiram Wood.

185. An Ancient sword, scabbard and belit. The blade of the sword appears to be of the best of steel, mounted with brass ; and on either side of the blade, in large capitals, is engraved the following : "God Bless the Province of New-York."

This sword was the property of a provincial officer who served in early French and Indian wars on the frontiers of New-York and Vermont, and who died at an advanced age more than fifty years ago. His property was sold at auction, and this sword was purchased by Mr. Francis Cobb of Cornish in Sullivan county, New-Hampshire (a remote connection of its former owner), and remained in his possession while he lived. Mr. Cobb died some two or three years since (about 1849), more than ninety years old; and when his property was inventoried, the sword was found among some rubbish in the attic of his house : it was purchased for a small sum, and presented to the New-York State Antiquarian Society by

ELEAZAR J JCKSON.

186. A Brick, made from the natural soil without any admixture. Brought from the city of New-York; being a specinen of the kind used in the erection of the new building on the southwest corner of Thames-street and Broadway, near Trinity Church, and called "Trinity Building": manufactured at Buffalo, Erie county.

R. Spencer Dyer. 


\section{PURCHASES.}

FROM WILLIAM C. HOSMER, OF AVON.

1. INDIAN ARRowheads(6), picked up in the ploughed fields in the vicinity of Avon, Livingston county.

2. Indian arrowheads of small size, used by indian boys in killing birds and inferior game(9). These arrows were inserted into the split end of tough wooden shafts, and fastened at the notches with ligatures of sinew or string bark.

3. INDian arrowheads(4), found on a plain near Fort Niagara, and of greater antiquity than those of flint formation.

4. Indian arrowheads(2), found on the farm of Timothy Hosmer, in the town of Porter, Niagara county, near the Lake shore; one of then curiously twisted.

5. A Hatchet-Shaped Flint, obtained from Col. Jewett of Lockport.

6. BROKEN ARrowheAds.

7. Stone DEERskin-dressers, found on the farm of Mr. Hurlburt, in the northeast part of the town of Avon.

8. Stone deerskin-dresser, found on the farm of Jeptha Wilber, Avon.

9. Stone DEERSKIN-DRESSER, found on the farm of James Wadsworth, near Borley's mill in the bend of the Conesus outlet, town of Livonia, Livingston county. The place where they were found was called "Fort Hill" by the early settlers; and mound, trench, and gateway were visible in the memory of men now living.

10. Stone pestle, used in pounding maize; found on the farm of Jeptha Wilber, Avon.

11. Ancient stone hatchet of rude construction, found on the site of the old village of Cannewangus (stinking waters), so called from its vicinity to the Mineral springs of Avon. After Denonville's invasion, the indians removed to the west side of the Genesee.

12. Ancient stone PIPE-Bowls(2), found in Rush, Monroe county.

13. Ancient stone pipe-bowls, found near Fort Hill : very rude specimens. 
14. Clay pipe-steins, found in the vicinity of Avon.

15. Clay pife-stems, found in Mendon, Monroe county, near the site of the village known in the seventeenth century to the French as "Dyen-de-haak-doh," or the Bend ; having been situated in an arm of the Hone-yah-yah (called by the whites Honeoyc), or the place where the finger was left. The tradition is, that an indian, while gathering strawberries on its banks, was bitten by a copperhead on one of his fingers ; and that to provent a spread of the venom, he severed it from his hand with a hatchet.

16. Fragments of ancient fottery, picked up at various points in the valley of the Genesee.

17. Ancient pottery found by Mr. Hosmer, together with a paint stone, in a mound of the Yemassees, near the ocean beach at San Pablo, on St. John's river, Florida.

18. Steel hatchet, found on the Wilber farm (Avon), in an old grave.

19. Bones, etc. taken from an indian grave near Fort Niagara.

20. SWORD-BELt PLATE, found near Cannewangus landing, by a workman on the Canal, many feet below the surface ; together with buman bones, and a fragment of a military coat that crumbled on exposure to the air. It is perforated by a bullet. It bears the inscription, “104, New-Brunswick Regiment," surmounted by the English crown. Some luckless British soldier, taken in some distant expedition of the conquering Iroquois, may have been the victim of Indian warfare.

21. Stone implenents, found on the farm of William Wadsworth near Fowlerville bridge, York, Livijgston county.

22. Paint stone referred to in No. 17. The bottom of the stone, when discovered, was red with vermilion or some of the coloring matters.

23. LEADEN CROSS, of Maltese shape, referring to missions of the Jesuits; and a bone rifle charger, found in a field that is known in song as "The Place of Bones." This field lies at the foot of a wooded ridge, in view of the village of Aron, and is supposed by some to have been the scene of conflict between Denonville and the Senecas.

24. French AXEs, found at an old Jesuit station on the Mendon road.

25. French axes, found on the site of a village near the dividing line between Avon and Lima, supposed to have been a famous town known to the Jesuits in the seventeenth century as Dyiu-don-sot, or Village at the spring. 
26. Decoy FIsh, used by the Sagenaw indians, Michigan, in winter. They drop the decoy through a hole cut in the ice, to entice the trout from his watery lair below : as he approaches the surface, the expert spearman on the watch easily secures the prize.

27. Fragments of Fire-Arms, picked up on the supposed battle-ground of Denonville.

28. Cannon bali found by workmen, while building a bridge at Jittleville, across the Conesus, four or five feet below the bed of the stream.

29. Teeth of animals, etc. found in an indian burial-place near the banks of the Genesee.

30. LaRge arrowhead und Stone deErskin-Dresser, found on the farm of F. M. Cutler, in a field south of Gore brook, Avon, bound with bark cord of indian manufacture.

31. Heavy dar of lead, stamp still visible; knife point ; fragment of a skull; stone deerskin-dresser, and fragments of brazen implements, found in graves in a sidehill on the farm of Richard Wilber, Avon. A bone cross, "lined and specked," was also found, but subsequently either lost or stolen. Below the depth penetrated by the ploughshare, ashes, charcoal, and charred kernels of maize are discovered in this hill of burial. The cross would indicate that it was formerly a Jesuit station.

32. BEADs of red pipe-stone; bone elegantly polished; glass; variegated shell, and brass and copper, found at different points of indian occupancy in the Genesee valley.

33. A SECTION of a circle, perforated near the rim with holes; fragment of some unknown implement of red pipe-stone, neatly cut and polished; and a large bead with a human head on one side of it, delicately carved and exquisitely proportioned : found in the old burial-place of Cannewangus.

34. Stone of octagonal shape, hollowed out : supposed to have been used by indian jugglers. Paint-stone? Found on the Street farm (so called), which is situated three miles from Avon, in a northwest direction, on the west side of the river, in Livingston county.

35. Stone chisel (in two pieces), used in excavating canoes. Found near Spanish hill, a few miles from Athens, Tioga county, which has been occupied for purposes of fortification, and indian traces alound. 
36. STONE DEERSKIN-DRESSER; and,

37. A Fragment of a pipe (as is supposed). Found near Fowlerville bridge, in the town of Avon, on a farm of W. W. Wadsworth, after the first ploughing of a new field, about one mile from the river bed, and three and a half miles in a southwesterly direction from Aron springs.

39. A Bone fieH-sfear, found on the Hurlburt farm in Avon, four miles in a northeast dircetion from the springs, at a place known to the inhbitants as Fort Hill : it was unquestionably a Jesuit station. The place was destroyed by Denonville in 1687. Corn, in a charred state, is found commingled with the subsoil. Bone crosses have been discovered, and rosaries; also many articles used by the French traders in Indian traffic.

39. A Stone implenent, with a handle like a mason's smoothing trowel. This implement was found near a spring on the farm of Ira Pierson in the town of Avon, about two miles in a southeast direction from the village of West-Avon.

Mr. JoHN GEBHARD junior states, that "this unique relic is made of the steatitic pyroxene of Prof. LewIS C. BECK (rensselaerite of Dr. EmMons); and from its strong resemblance to the specimens of that mineral from Edwards, St. Lawrence county, the material from which it is constructed is undoubtedly from that locality. It was probably used in dressing deerskins, and for smoothing and softening the seams in manufacturing moccasius and other articles made from skins. It evidently belongs to the ante-columbian period."

40. VARIous beads. These beads were found in various places in the valley of the Genesee : at the Jesuit stations previously referred to; the old indian burial-place, near the red bridge that crosses the river one mile west of West-Avon village; and a few were found near Fort Niagara, and in the valley of the Susquehannah.

41. Stone Deerskin-dresser. Found on the farm of Francis M. Cutler, in West-Avon.

42. Stone deErghin-presser. Found on the Wilbur farm.

FROM E. G. SQUIER, OF NEW-YORK.

43. Fltnt arrowheads From Cayuga county, 7 specimens; Monroe county, 2 ; Livonia, Livingston county, 2 ; vicinity of Buffulo, Erie county, 1; Ellisburgh, Jefferson county, 1 ; and 4 from localitics not named. 
44. French axes(2). From Cayuga village, Cayuga county.

45. Indian pestle. From Cayaga county.

46. Copper hetrle. From an indian grave in Scipio, Cayuga county.

47. Gunbarrel. From the site of Denonville's battle with the Senecas (1687), near Victor, Ontario county.

48. Scalfing-rinife. From the grave of a Cayuga warrior in Scipio, Cayuga county.

49. Stone axes(4), From Springport, Cayuga county; Adams, Jefferson county; Ellisburgh, Jefferson county; and one locality not named.

50. Fragments of pottery. From the site of a Seneca village, Livonia, Livingston county.

51. Fragments of pottery. From the site of an old Seneca village in Mendon, Monroe county.

52. Pipes, pottery ( 5 bozes). From an ancient enclosure in the town of Ellisburgh, Jefferson county.

53. Pipes, pottrey, \&c. ( 4 boxes). As No. 52.

54. Terra-cottas. From Leroy, Genesee county, 2 specimens; Ellisburgh, Jefferson county, 7; Scipio, Cayuga cuunty, 1; and locality not named, 1.

55. Potreny. From Ellisburgh, Jefferson county, 3 specimens; and from an ancient village of the Senecas, Livonia, Livingston county, 1 .

56. Copper rnives, and other metallic articles. From an old Seneca village, Livonia, Livingston county.

57. Potterv. From an ancient enclosure in Jefferson county, 2 specimens; from Scipio, Cayuga county, 2; and from Livonia, Livingston county, 2.

58. Various articles of pottery ( 2 boxes). From an enclosure or mound near Buffalo.

59. Various articles of potter $\mathrm{Y}$. From a large mound on Tonawanda island, in Niagara river : excavated by E. G. Squier, Novem. ber, 1848.

60. Homan remains. From the great mound on Tonawanda island.

61. Bone implements. From Ellisburgh, Jefferson county, 3 specimens; from Leray, Jefferson county, 1.

62. Stone axes. From Buffalo, 1; Livonia, Livingston county, 1; Ellisburgh, Jefferson county, 1.

63. Deposits from altar mounds of the Mississippi valley.

64. Deposits from sepulehral mounds of the Mississippi.

65. Mortar, from the old tower at Newport, Rhode-Island, 
FROM LEWIS H. MORGAN, OF ROCHESTER.

ARTICLES MANUFACTURED AT SPECIAL REQUEST, BY INDIANS RESIDING IN WESTERN NEW YORK.

The name of each article is in the Seneca dialect of the Iroquois language. .

[ $\ddot{\mathrm{a}}$ is sounded as in $\operatorname{arm} ; \breve{\mathrm{a}}$, as in at; a, as in ale.]

66. Gü-no-jo-o : INDIAN DRUM (3 varieties). Used in dances.

67. Gus-dä-wa-să : Turtle-shell rattle (2 specimens). Used in dances.

68. Gus-dä-wa-să : SquAsh-shell rattle (4 varieties). Used in dances.

69. Grus-dä-wa-să Yen-che-no-hos-ta : KNEE ratTle, of deer hoofs (one pair). Used in dances.

70. Gü-gch-tä Yen-nis-hä-hos-ta : Arm-Bands (1 pair).

71. Yen-nis-ho-quä-hos-ta : Wristbands (1 pair).

72. Gü-geh-tä Yen-chc-no-hos-ta-ta : KNEE-BANDs (1 pair).

73. Gä-geh-tä : INDIAN BELT (3 varieties).

74. Ah-tä-quä-0-weh : Moccasin, for male (1 pair).

75. Ah-tä-quä-0-weh : Moccasin, for female ( 1 pair)

76. Gä-kä-ah : KILT or SKIRT, worn in war dance.

77. Gä-kä-ah : KILT or sKIRT, worn by indian women.

78. Gise-hă : LegGin, for male (1 pair).

79. Gise-hă : LEGgIN, for female (1 pair).

80. Gä-swhen-tä : NEckLace.

81. Ya-wa-o-dä-qua : Pincushion (3 varieties).

82. Gä-yä-ah : WorkBAG (5 varieties).

83. Got-gwen-dä : Pocketbook (6 varieties).

84. Gä-kä : Brfechcloth. Used in ball-game, foot-race, etc.

85. Gä-de-us-ha : W AmPUM NEcKLAce. Da-yu-yä-sont, name of a cross.

86. Ya-wa-o-dä-quä : Needueboors (5 varieties).

87. Ga-on-seh : BABY FRAME.

88. Gä-0-wä : Bark Tray (3 specimens).

89. Ah-de-gwas-hă : Hominy BLAde, or Soup-sTick (4 specimens).

90. Ah-was-hă : Earring (1 pair).

91. Gä-jih : BowL, for a game with peachstones.

92. Gus-ka-eh : Peachstones (6 specimens).

93. Gus-ga-e-sa-tä : DeEr Butrons, for an indian game (8 specimens, or one set).

94. Gä-geh-dä : Javelin or Shooting-sticK, for an indian game (18 specimens).

95. Yun-ga-sa : Toвacco Pouch (4 specimens). 
96. Gä-ne-gä-tä : Mortar, for pounding corn (2 specimens).

97. Gä-nih-gä-dï : Mortar pounder (2 specimens).

98. Gä-ne-ah : BaLl BAT, used in playing an indian game (4 specimens).

99. Gä-wä-sä : Snowswake (4 specimens).

100. Gä-je-wä : WAR-CLUB, with ball head (4 specimens).

101. Gä-neu-ga-o-dus-ha : WAR-CLUB, with deer-horn tooth (2 specimens).

102. O-sque-sont : Tomahawk.

103. Ah-so-quä-ta : Pipe (made from a cyathophyllum).

104. Wä-a-no : INDIAN Bow (6 specimens).

105. Gä-no : Arrow (50 specimens).

106. Gä-go-shä : FALSE FACE.

107. Gä-weh-ga-ă : SNowshoe (3 pairs).

108. O-tä-quă-osh-ha : SNowshoe, of splint (1 pair).

109. Gä-sken-dä : BARK ROPE, made of slippery elm.

110. Gus-hä-ah : Burthen-strap, made of slippery elm.

111. Gus-hä-ah : Burthen-strap, made of basswood.

112. Ose-gä : Srein of slippery elm strings.

113. Ose-hä : SkEIN of basswood-bark strings.

114. Ah-da-dä-quä : INDIAN SADdLE.

115. Gä-na-quä : BaRK Barrel. Used for beans, dried corn, etc.

116. Gä-o0-wă : BARK sAP-тUB (3 speeimens).

117. O-nus-quä Ah-hose-hă : KNot BaLI (2 specimens). Used in playing a game.

118. O-no-neä Gos-ha-dä : HusK salt-Bottle (2 specimens).

119. O-je-she-wä-tä : CAKE of deer's brains and moss, for tanuing deerskins.

120. Gä-nuh-sä : BreastPLATE of shells.

121. Got-kase-hä̈: Axehelve.

122. Gä-ga-an-dä : AIr-GUN.

123. Dä-ya-yä-dă-gä-ne-at-hä : Bow and wHEEL for striking fire.

124. Gä-gis-dä : STEEL, FLINT and PUNK, for striking fire.

125. Gis-tak-he-ä : SKIN BAG (speckled fawn).

126. Gis-tak-he-ä : SKin bag (bearskin).

127. Tuesh-tï-ga-tas-tä : Tin breastplate.

128. Skä-wä-ka : Splint BRoom.

129. Ya-o-dä-was-tä : Indian Flute.

130. Ne-us-tase-ah : BASKET sIEvE. Used for sifting white corn.

131. O-ne-ose-to-wa-nes : BasKet sieve, coarser; for white-flint corn.

132. Ta-gase-hă : MARKET вASKET.

133. Gase-hă : Covered basket.

134. O-gä-kä-ah : OPEN-WORK BAsKET (3 specimens).

135. Ga-yuh : Splint cradle. 
136. Gü-nose-hä : HUSK and FlaG Basket (4 specimens).

137. Ya-nuh-ta.dä-quä : Tollet basket.

138. O-gus-ha-ote : Small square basket (17 specimens). These baskets are numbered from 1 to 17 inclusive, and contain specimens of the several varieties of corn, beans, squashes, tobacco, dried corn, etc. raiscd and prepared by the Senecas, viz :

I. O-na-0-ga-ant : WHITE CORN.

II. Tic-ne : Red corn.

III. Ha-go-wä : White-FLINT coRN.

IV. O-nä-dä : Charred, or Roasted corn.

v. O-go-ou-să : BAKEd cORN.

vi. O-si-dä : Long-vine BEAN.

viI. Gweh-dä-ă O-si-dä : Red bean.

vIIr. Te-o-gä-ga-wă O-si-dä ; SpeckLEd bEAN.

Ix. Ta-gä-gä-hät : ShorT-vine bean.

x. Ah-wa-own-dä-go : RED-FLowER POLE-BEAN.

XI. Hä-yoke : Cranberry polez-bean.

XII. O-gä-gä-ind : GRAY SQUASH.

xiII. Gä-je-ote : Birs-handLe squash.

xiv. Sko-als: TOAD SQUASH.

xv. O-ne-ä-sä-ä-weh : SMaLl sqUash.

xvi. O-yeh-quä-ä-weh : Iñdian tobacco.

Xvir. O-s0-Wa: PARChed Corn pounded into flour, with maple sugar.

139. Gä-no : Akrow for air-gun (2 specimens).

140. O-sque-sont : Tomahawr. Used in the bear hunt.

141. Da-ya-no-a-quä-tä Gä-ga-neä-sä : ScALPING-KNIFE (2 specimens),

142. O-na-o-ga-ant : Two Ears of white corn.

143. Tic-ne : Two ears of red corn.

144. Ho-go-wä : Two EARS of white-flint corn.

145. Gus-to-weh : Her.D-Dress.

146. Gä-ger-we-sä Dun-daque-quä-do-quä : Newyear's shovel.

147. To-do-war-she-do-wä : RiBbon for hair.

148. Gä-de-us-ha : Necklace.

149. De-con-deä-da-hust-tă : BELT for female costume.

150. Ah-de-a-dä-we-să : Female uPPER DREss, with silver broaches, etc.

151. Dä-yase-ta-hos-ta : Silver hatband.

152. Yen-nis-ho-quä-hos-ta : Silver wristbands ' 1 pair).

153. To-an-jer-go-o 0-no-no-do : Groundnuts (Apios tuberosa), from Tonawanda. 
ARTICLES MANUFACTURED AT SPGCIAL REQUEST, BY INDIANS RESIDING IN CANADA WEST.

154. Gä-däs-hă : SHEAF for carrying arrows.

155. Gä-je-wä : WARCLUd, with ball head.

156. Gä-such-tä Ote-lko-ă : BeLt of WampUm.

157. Ote-ko*ă : STrRING of WAMTPUn.

158. Ah-so-quä-tä : STONE PIPE.

159. Da-gä-yä-sont : Silver cross, 8 inches by 5 .

160. Da-gä-yä-sont : do 6 inches by 4 .

161. Da-gä-yä-sont : do 3 inches by $1 \frac{1}{2}$ ( 2 specimens).

162. Au-ne-as-gă : Silver broach, 4 inches diameter.

163. Au-ne-as-gă, : do 3 inches diameter.

164. Au-ne-as-gă : do $\quad \frac{1}{2}$ inches diameter. There are in all

13 broaches of various sizes.

165. Au-ne-as-gă : Silver broACH (2 specimens).

166. Au-ne-ä-hus-hă : Finger-RING (4 specimens).

167. Ah-was-hă : EArRINGs (1 pair).

168. Dä-yase-ta-hos-ta : Silver hatband.

169. Ont-wis-tä-ne-un-dä-quä : Silver beads (long).

170. O-wis-tä-no-o O-sta-o-quä : Round silyer beAds (variety).

171. Gă-ose-hă : Baby firame.

172. Gä-swä-hos-hă : BABY-FraMe BeLT.

173. Gă-nose gă : Baby-Frame BELT (2 specimens).

174. Da-ya-he-gwä-hus-ta : HATBANd of BROACHES.

175. Gä-yä-ah : SA TCHEL.

176. Gä-ya-äh : WовкваG.

177. Ya-wa-o-dä-qua: Pincushion (2 specimens).

178. Gä-kä-ah : KILt, made of fawn skin.

179. Got-ko-on-d:i Gise-hă : DeERskin LEgGin.

180. Da-yunt-wä-hos-tä : DeErskin waIsT-beIt.

181. Yunt-ka-to-dä-tä : DeERskin shouldER-BeLtr.

182. Ah-tä-quä-0-weh : Moccasin (3 specimens).

183. Hair ornament.

184. Sнот-Родсн.

185. Gät-go-ne-as-heh : Hommony bLadE (2 specimens)。

186. Gät-go-ne-as-heh : do a chain cut on the end of the handle.

187. Ya-ă-go-jen-ta-quä : BREAD-TURNER.

188. Ah-do-gwä-seh : WOODEN LADLE (4 specimens).

189. Ah-do-gwä-seh : BARK LADLE. 
190. Ah-do-gwä-seh : WOODEN SPOoN.

191. Gä-na-quă : BarK barrel (3 sizes).

192. Gä-o-wä : BARK TRAY (6 sizes).

193. Gä-00-wä : BARK sAP-TUb.

191. Gănte-as-hă : GLAss BeAds.

195. Gă-no-sä : Conch-shell bReast-plate.

196. Ah-dä-dis-hă : C CANE (2 specimens).

197. Ah-so-quä-tä : PIPE, made of Missouri stone.

198. Ah-so-quä-tä : PIPE, made of black stone.

199. Ah-so-quä-tä : PIPE, made of nodule.

200. Ah-so-quä-tä : PIPE, made of wood and lead.

201. Gü-gä, ne-as-heh : Belt and кNife.

202. Gus-dü-wah-să : Turtleshell rattle (2 specimens).

203. O-no-gä Gus-dä-wah-să : Horn rattle.

204. Gus-to-weh : Headdress.

205. Gä-wă : Moccasin AwL.

206. Bunch of sumac.

207. O-yeh-quä-ä-weh : INdiAN товAcco.

208. Gis-tät-he-0 Gä-yä-ah : Fawnskin Bag.

209. Gus-dä-wa-sä Yen-che-no-hos-ta : KneE-Rattle, of deer-hoofs.

210. Spear used in the war of 1812.

211. Gä-ne-a-ga-o-dus-ha: WAR-cLUB.

212. Ya-0-dä-was-tä : Indian FLUTE (2 specimens).

213. Wä-a-no : INDIAN kow.

214. Gä-ne-ah : BaLL-BAt.

215. Gä-no: Arrow, pointed with deer's horn.

216. Gä-no : FEATHERED ARRows (18 specimens in sheaf).

217. O-dä-da-one-dus-tä : EYE-sHOWERER.

218. Yun-ga-sa : Tовассо-рочсн, made of the foot and leg of the snapping turtle.

21.9. Gä-go-sä : FaLSE face.

220. O-ä-ta-ose-kä : Moosehair bURthen-strap.

221. Gus-hä-ah : Moosehair and bark bURthen-strap.

222. Gus-hä-ah : Burthen-STrap (bark thread and worsted).

223. Gus-hä-ah : BURTHEN-sTRAP (bark, with moosehair figures).

224. Gä-te-äs-hă Gä-a-o-tä-ges : Grass sHoUlder-ornament.

225. Yout-kä-do-quä : BASKET FISH-NET.

226. Husk moccasins (one pair).

227. Gü-a-sken-dä : BARK ROPE, made of basswood filaments.

228. Gä-a-sken-dä : BARK ROPE (2 specimens from Tonawanda).

229. Ose-gă : Skein of sLifPert-elmi thread.

230. Osc-ğ : Skein of ditto, colored. 
231. Ose-gă : Twisted into strings.

232. Ose-gă : Strips of SLIPPERy elim bark.

233. O-să : Skein of Basswood FiLAMENTs.

234. O-să : STRIPs of RASSWOOD BATK.

235. Go-yo-ga-ace : Finger-CATCHER.

236. BIRD-TRAP, for catching quails.

237. Gus-hä-ah : DeErhair burthen-strap.

238. Gus-hä-ah : Basswood bURthen-strap.

239. Ne-us-tase-ah : Basket sieve,

240. Gase-hă : Covered Basket.

241. O-gä-lrä-ah : OPEN-WORK BASKET.

242. Ta-gase-hă : Mariet basket.

243. Gä-geh dä ; JaVeLin, or Shooting-stick.

244. Ah-de-gwas-hă : Hominy bLade.

24.5. Paddles (6 specimens).

246. Yun-des-ho-yon-dä-gwat-hä : Pop-corn steve.

247. An-ne-us-gă : Silver вrоACH (9 specimens on a card).

248. An-ne-us-gă : do (20 small specimens on a card).

249. Gä-lia-ah : SkIRT.

250. Gise-hă : Leggins for female (one pair).

251. Ah-de-a-dä-we-să : Female overdress.

252. E-yose : Broadcloth BLANKET.

253. O-sta-o-quä : BEAD NEcKLACE.

254. Gä-ka-ah : Skirt for female.

255. Gise-hă : LEGGINs for female (one pair).

256. Ah-de-a-dä-we-să : Overdness for child.

257. Yen-nis-ho-quä-hos-ta : Wristbands of bEads (one pair).

258. O-ha-dä : PorcưINe Quills.

259. DEER'S HATR, used for making burthen-straps, etc.

260. Da-ya-no-tä-yen-dä-quä : SNow-Boat.

261. Gä-wä-sä : SNow-SNaKE (5 specimens).

262. Gü-ne-ko-wă-ah : Burthen frame.

263. Gä-no-sote : BaลK HoбsE.

264. Gä-snä Gä-o-no : BaRK CANoE.

\section{FROM VARIOUS PERSONS.}

265. Gänyä-ah : WorkBaG (2 specimens).

266. Da-ya-he-gwă-hus-tä : HAtband of BROACHEs.

267. An-yus-gă : Silver broACH (large variety).

268. An-yus-gă : Silver BRoACH (smaller variety). 
269. An Indian PIPE, manufactured from the steatilic pyroxene of Prof. Lewis C. Beck. Found near Fort George, Warren county. This relic presents the best specimen of indian design and sculpture ever found in this State. It is threo inches in height, and represents an indian in a sitting posture, with his arms resting upon his knees. The back constitutes the bowl of the pipe, at the lower extremity of which there is a hole for the insertion of the stem.

270. An Indian ReLic, plowed up at the depth of a foot beneath the surface of the soil, in the town of Commerce, Oakland county, Michigan, in the year 1848.

This relic is made of novaculite, and in the form of an axe with two edges, with a hole in the centre for the insertion of a handle.

271. Tomahawr, which formerly belonged to the celebrated Indian Chief Cornplanter. 


\section{COLLECTION}

OF THE LATE

\section{H. CASIMIR DE RHAM, JUNIOR:}

PRESENTED BY HIS PARENTS,

MR. AND MRS. H. C. DE RHAM. 



\title{
THE DE RHAM COLLECTION.
}

\author{
MAMMALIA.
}

\section{ORDER CARNIVORA.}

FAMILY SORECIDA.

Latin Names.

English Names.

1 Condylura czistata. Starnose Mole (male, female, and two young).

2 Sorex DEKayi.

Dekay's Shrew.

FAMILY URSID床,

3 Procyon Lotor.

Raccoon.

FAMILY MUSTELIDAE.

4 Mefhitis amertcana. Skunk.

5 Mustela fusca. Brown Weasel.

6 Putorius noveboracensis. New-York Ermine.

7 Putorius vison. Mink.

FAMILY CANID正.

8 Vulpes fulvus. Red Fox.

FAMILY FELIDA.

9 Lyncus BoREALIs. Northern Lynx.

\section{ORDER RODENTIA.}

FAMILY SOIURID $\approx$.

10 Sciurds leucotis.

11 Sciorus vulpints.

12 Sciurus Niger.

13 Sciurus hudsonicus.

14 Sciurus Hudsonicus.

15 Sciurtis striatus.

16 Pteromys volucella.
Little Grey Squirrel (male and female). Fox Squirrel.

Black Squirrel (male and female).

Red Squirrel (male and female).

Red Squirrel (albino).

Striped Squirrel.

Flying Squirrel. 


\section{FAMILY ARCTOMIDA.}

Latin Names.

English Names.

17 Arctomys monax.

Woodchuck.

FAMILY GERBILLIDÆE.

18 Meriones americanus. Deermouse.

FAMILY CASTORIDAS.

19 Fiber zibethicus. Muskrat, or Musquash (m. and f.).

FAMILY MURIDA.

20 Mus leucopts.

Jumping Mouse.

21 Arvicola rufescens. Tawny Meadow-mouse.

FAMILY LEPORIDA.

22 Lepus nanus.

American Grey Rabbit.

23 LEPUS AMERICANUS.

Northern Hare (m., f. and y.).

24 Erinaceus europdeus. Hedgehog (old and young), from England,

25 CAPra ibex (Linneus). Skull and horns of the male, from the Alps.

\section{BIRDS.}

\section{ORDER ACCIPITRES.}

IAMILY VULTURID $Æ$.

1 Cathartes gryphus. Condor (male), from the Andes.

2 Cathartes aura. Turkey Buzzard (male).

FAMILY FALCONIDAE.

3 Aquila chrysetos. Golden Eagle (male).

This fine specimen was shot a $\mathrm{fcw}$ years since at Islip, Suffolk county, and is the identical one referred to by DekaY in his Zoology of New-York, Part II, page 4.

4 Halizes lescocerphalus. Bald Eagle (male and female).

5 Pandion carolinensis. American Fish-haruk (male), with a fish in the bealk.

6 Buteo sancti-joannis, Rough-legged Buzzard (m. and f.). 
Latin Names.

7 Buteo borealis.

8 Nauclerus furcatus.

9 Falco columbarius.

10 Astur atricapillus.
English Names.

Red-tailed Buzzard (male). Swallow-tailed Hawk (male). Pigeon Hauk (male and female). American Goskawk (male).

\section{FAMILY STRIGID纽,}

11 Surnia funetrea.

12 Surnia nyctea.

13 Bubo asio.

14 Otus palustris.

15 Ulula nebulosa.

16 Ulula acadica.
Hawk Owl.

Snowy Owl (male).

Little Screech-owl (male and female). Short-eared Owt (male).

Barred Owl (male and female). Acadian Owl (male).

\section{ORDER PASSERES.}

\section{FAMILY CAPRIMULGIDAN.}

17 Caprimulgus vociferus. Whippoorwill (male).

\section{FAMILY HIRUNDINID $\mathbb{E}$.}

18 Hirundo purpurea.

19 Hirundo fulva.
Purple Martin (male).

cliff Swallou (male and female).

FAMILY AMPELID五.

20 Bonbzcilla carolinensis. Cedar-bird (female).

\section{FAMILY ALCEDINID $A$.}

21 Alcedo alcyon.

Belted Kingfisher (young male).

\section{FAMILY CRRTHID A .}

22 Troglodytes enon. House Wren (male).

23 Troglodytes ludovicianus. Mocking Wren (male).

24 Troglodytes palustris. Marsh Wren (male).

\section{FAMILY PARIDA.}

25 Partus bicolor.

Crested Tit (two males).

\section{FAMILY் SYLVIAD正.}

26 Reguldis satrapa.

27 Sialia WILSONI,
Golden-crested Kinglet (male).

Bluebird (male and female). 


\section{FAMILY MERULID出.}

Latin Names.

28 Orpheus polyglottus.

29 Orpheus rufus.

30 OrpheUs Carolinensis.

31 Merula migratoria.
English Names.

Mockingbird (male).

Brown Thrush (male).

Catbird (male and female).

American Robin (nale and female).

\section{FAMILY SYLVICOLID AE.}

32 Trichas philadelphia. Mourzing Warbler (male and female.

33 Vermivolia pennsylvanica. Worm-eating Warbler (m. and f.).

34 Vermivora solitaria. Blue-winged Warbler.

35 Vermivora chrysoptera. Golden-winged Warbler (male).

36 Vermivora peregrina. Tejnessee Warbler (male).

37 Vermivora celata.

38 Sylvicola mactilosa.

Orange-crowned Warbler (young male).

39 Sylvicola pardaltía. Spotted Warbler (male).

40 Sylvicola blachburnia. Blackburnian Warbler (male).

41 Sylvicola castanea. Bay-breasted Warbler (male).

42 Sylvicola striata. Blackpoll Warbler (male).

43 Sylvicola discolor. Prairie Warbler (male).

44 Sylvicola canadensis. Black-throated Blue Warbler (young mile).

45 Sylvicola erstiva. Summer Yellowbird (male and young).

46 Sylvicola pinus. Pine Warbler (female).

47 Sylvicola icterocephaj,A, Chestrut-sided Warbler (male).

48 Sylvicola parus.

49 Sylvicola formosa.

50 Wilsonia mitrata.

51 Wilsonia pusilia.
Hemlock Warbler (two males).

Kentucky Warbler (male).

Hooded Warbler (female).

Green Black-capped Warller (male).

\section{FAMILY MUSCICAPIDA.}

52 Culicivora cerrulea.

53 Musctcapa rtticilla. 54 Tyrannus crinitus.
Blue-grey Gnatcatcher (male). American Redstart (male and female). Great-crested Kingbird (male).

\section{FAMILY VIREONIDA.}

55 Vireo olivaceus. 56 ICteria VIRIDIS.
Red-eyed Greenlet (male).

Yellow-breasted Chat (male).

FAMILY LANIIDA.

57 Lanius septentrionalis. Northern Butcherbird (two males). 


\section{FAMILY CORVIDA.}

Latin Names.

58 Garrulus cristatus.

59 Garrulus canadensis.

60 Pica caudata.

61. Corvus americanus.

62 Corvus corax.
English Names.

Blue Jay (male).

Canada Jay (male).

Magpie (male).

Cornmon Crow (male).

Raven.

\section{FAMILY QUISCALIDAE,}

63 Quiscalus versicolor. Common Crow Blackbird (m. and f.).

64 Sturnella ludoviciana. Meadow Lark (male and female).

65 ICterus baltimore.

Golden Oriole (male).

66 ICterus pheniceus.

Red-winged Oriole (male).

67 Molothrus pecoris.

Cow Bunting (female).

68 Dolichonyx oryzivorus, Bobolink (male and female).

\section{FAMILY FRINGILLID_E.}

69 Coccoborus ceruleus. Blue Grosbeak (male).

70 Coccoborus ludovicianus. Rose-breasted Grosbeak (male).

71 Struthus hyemalis. Snozbird (male and female).

72 Fringilla iliaca. Fox-colored Sparrow (male).

73 Fringilia pennsylvanica. White-throated Sparrow (m. and f.).

74 Fringilda leucophrys. White-crovened Sparrow (male).

75 EMberiza savanNa.

Savannah Bunting (female).

76 Ammodramus palustris.

Swamp Finch (female).

77 Carduelis tristis.

Yellowbird (male).

78 Erythrospiza purpurea. Crested Purple-finch (male and ferale).

79 Pitylus cardinalis.

Cardinal Grosbeak (male).

80 Pipilo ertithrophthalmus, Chewink, or Ground Robin (male).

81 SPIZA CXaNEa.

Indigo-bird (male).

82 Pyranga estiva.

Redbird (male, young male and female).

83 Pyranga rubra. Black-winged Redbird (male).

84 Plectrophanes lapponicus. Lapland Snowbird (female).

85 Plectrophanes nivalis. White Snowbird (male and female).

86 Alatdda cornuta.

Horned Lark (male and female).

87 Cortthus enucleator.

88 Loxia leucoptera.

Pine Bulfinch (male, young $\mathrm{m}$. and $\mathrm{f}$ ).

White-uinged Crossbill (male).

FAMILY PICID压.

89 Picus pileatus.

Crested Woodpecker (female).

90 Picus erythrocephalus. Redheaded Woodpecker (male). 
Latin Names.

91 Picus villosus.

92 Picue pubescens.

93 Picus varius.

94 Picus carolinus.

95 Picus arcticus.

96 Picus auratus.
English Names.

Hairy Woodpecker (male).

Downy Woodpecker (male).

Yellow-bellied Woodpecker (male).

Red-bellied Woodpecker (male).

Arctic Woodpecker (male).

Golden-winged Woodpecker (2 males and 1 fem.).

FAMILY COLUMBID平,

97 Ectopistes migratoria. Wild Pigeon (male).

ORDER GALLIN়录.

FAMILY PHASIANIDAE.

98 Meleagris gallopavo. Wild Turkey (male).

Also young of the domestic fowl.

FAMILY TETRAONIDAE.

99 Ortux virginiana.

American Quail (m., f., and 3 young).

100 Tetrao ungelius.

Common Partridge (m., f., \& 3 young).

101 Tetrao curido.

Pinnated Grouse (male and female).

102 Tetrao canadensis.

Spruce Grouse (male and female).

103 Argus giganteus (Temminck). Argus Pheasant. From Sumatra.

104 Phasianus nycthemerus (Linneus). Silver Pheasant. From China,

ORDER GRALLÆ,

\section{FAMILY CHARADRID 2 .}

105 Charadrius semipalmatus. American Ring Plover (male and fem.).

106 Charadrius melodus. Piping Plover.

107 Charadrius vociferus, Kildeer Plover (male).

108 Charadrits virginicus. Golden Plover (male and female).

109 Strepsilas interpres. Turnstone:

\section{FAMILY GRUID AE.}

$110 \Lambda$ aDEA Herodias. Great Blue Heron (adult with a fish in the beak, and an immature male).

111 ARdea lUdoviciana.

112 Ardea yirescens,
Louisiana Heron (male),

Green Heron (male and female). 
Latin Names.

113 Ardea exilis.

11.4 ARdea minor.

115 Ardea discors.
English Names.

Small Bittern (male). American Bittern (male and female). Black-crowned Night-heron (male).

\section{FAMILY SCOLOPACIDAE.}

116 Numentus longrirostris. Long-billed Curlew (male).

117 Numenius hudsonicus. Jack Curlew (male).

118 Heteropoda semipalitata. Semipalmated Sandpiper (male).

119 Tringa marttima.

120 Tringa rufescens.

121 Tringa cinclus.

122 Tringa schinzi.

123 Tringa hectoralis.

124 Tringa canutus.

125 Tringa pusilia.

126 Calidris arenaria.

127 Totanus macularjus.

128 Totanus bartramius.

129 Totanus flavifes.

130 Totanus melanoleucus.

131 Totanus semipalmatus.

132 Scolopax WILsoni.

133 Rusticola minor.
Purple Sandpiper (male).

Buff-breasled Sandpiper (female).

Black-breasted Sandpiper (male).

Schinz's Sandpiper (male).

Pectoral Sandpiper (male and female).

Redbreasted Sandpiper (male and fem.).

Witson's Sandpiper (male).

Sanderling (two males and one female).

Spotted Sandlark (male).

Grey Plover (male).

Yellowlegs (male).

Varied Tatler (male and young).

Willet (male).

Common American Snipe (2 m. and f.).

American Woodcock (m., f., \& 2 young).

FAMILY RALLID AE.

134 Rallus crepitans.

Saltwater Meadowhen (male).

125 Ortygonetra carolina. Sora Rail (male).

136 Galinula galeata. Florida Gallinule (male).

FAMILI் RECURVIROSTRID $\approx$.

137 Himantopts nigricolis. The Lawyer (male and female).

\section{ORDER LOBIPEDES.}

FAMILY PODICIPID $Æ$.

138 Podicefs nubricolis. Rednecked Grebe (male).

139 Hydrota carolinensis. Dipper, or Pied Dobchick (male). 


\section{ORDER NATATORES.}

\section{FAMILY ALCIDAE.}

Latili Names.

140 Mergulus alie.

141 AlCa torda.
English Names.

Sea Dove (malc).

Razurbill (male).

FAMILY COLYMBIDA.

142. Colymeus glacialis. Great Loon (male and femalc).

143 Colymbus septentrionalis. Red-throated Loon (female).

FAMILY PROCELLARID E.

144 Thalassidroma wilson. Wilson's Petrel (male).

\section{FAMILY PELECANIDA.}

145 Suda a mericana.

American Gannet (male).

\section{FAMILY LARID $A$.}

146 Rhynchors Nigra.

147 Sterna hirundo.

148 Sterna caYana.

149 Sterna Nigra.

150 LaRUs argentatus.

151 LARUS ATRICILLA.

152 Larus bonapartil.

153 LaRUs sabini.

154 LESTRIS BUFFonI?

155 Lestris RICHARDsonit.
Black Skimmer (male).

Common Tern (male).

Cayenne Tern (male).

Black Tern (male and young).

Winter Gull (male and female).

Laughing Gull (male).

Bonaparte's Gull (male and female).

Fork-tailed Gull (male).

Arctic Hawk-gull (malo).

Richardsnn's Hawh-gull (male).

\section{FAMILY ANATIDA.}

156 Mergus merganser.

157 Mengus cucullatús.

158 Fuligula valisneria.

159 Fuligula marila.

160 Fuljgula rufitorques.

161 Fuligula labradora.

162 Fuligula rtjitda.

163 Fuligula glacialis.

164 Fitligula albeola.

165 Fuligdla clangula.

166 Fuligula histrionica.
Buff-breasted Sheldrake (male).

Hooded Sheidrake (male and female).

Canvasback (male).

Broadbill (female).

Bastard Broadbill (male).

Pied Duck (male).

Ruddy Duck (male and female).

Oldwife (male in winter plumage, and

- male and female young).

Buffelceaded Duck (male and female).

Whistler (male).

Harlequin Duck (male). 
Latin Names.

167 Fuligula molissma.

163 Fuligula perepicillata.

169 Fuligula americana.

170 Fuligula fusca.

171 ANas sPONSA.

172 ANAS CAROLINENSIS.

173 Anas aCUTa.

174 Anas clypeata.

175 Anas strepera.

176 ANas americaNa.

177 ANSER CANADENSIS.

178 ANSER ALBIFRONS.

179 ANSER BERNICLA.
English Names.

Eider Duck (male).

Surf Duck (immature male).

Broadbilled Coot (male).

White-winged Coot (male).

Wood Duck (male and young male).

Green-uinged Teal (male and female).

Pin-tailed Duck (male and female).

Shoveller (male in winter plumage, and male and female in summer plumage).

Grey Duck, or Gaduall (male).

Anerican Widgeon (male and female).

Wild Goose (male).

White-fronted Goose (male).

Brant Goose (male).

\section{AMERICAN BIRDS DESCRIBED BY AUDUBON, WHICH}

HAVE NO'T BEEN OBSERVED IN THE STA'TE OF NEW-YORK.

180 Ictinia plumbeus.

181 FaLCo perEgrinus.

182 Milvulus tyrannus.

183 Helinaia protonotarius. Prothonotary Swamp-warbler (m.\& f.).

184 Sialia occidentalis.

185 Sialia arctica.

186 Turdus næivius.

187 Coccothraustes vespertina. Evening Grosbeak (male and female). 188 Pyranga ludoviciana. Louisiana Tanager (male and female).

189 ICterus bULLOCKit.

190 Garrulds stelleri.

191 Sitta pusilla.

192 Picus princtpalis.

193 Picus Querulus.

194 Picus torquatus.

195 Picus mexicanus.

196 Centurus carolinensis.

197 Columba Passerina.

198 ORTYX PLUMIFERA.

199 IBIS RUBRA.
Mississippi Kite (male).

Great-footed Hawk (male, with Golder. winged Woodpecker under foot).

Fork-tailed Flycatcher (male).

Western Bluebird (male).

Arctic Bluebird (male).

Varied Thrush (female).

Bullock's Hangnest (male).

Steller's Jay (male).

Brown-headed Nuthatch (male).

Ivory-billed Woodpeckier (male and fem.). Red-cockaded Woodpecker (m. and f.).

Lewis's Woodpecker (malc).

Red-shafted Woodpecker (male).

Carolina Parrot (young).

Ground Dove (male).

Plumed Partridge (male).

Scarlet Ibis (male). 


\section{FOREIGN BIRDS.}

Latin Names.

English Names.

200 Upupa epops, Linneus. Hoopoe. Africa.

201 Erimachus magnificus, Oken. Rifleman (male and female). New South Wales.

202 Oriolus galbula, Oken. Golden Blackbird. Europe.

203 Gracula rosea, Oken. Rose-colored Thrush. East Indies.

204 Edolius malabaricus, Oken. Drongo. Africa.

205 Galbulus viridis, Olsen (two specimens). Africa.

In addition to these, there are twenty-two specimens of foreign birds whose specific names have not been determined.

\section{MINERALS, FOSSILS, SHELLS, \&c.}

\section{ROCKS.}

1 to 50 inclusive. Rock srecimens, from Cumberland.

\section{MINERALS.}

51 Carbonate of barytes, incrusted with copper pyrites and carbonate of lime.

52 Crystalitzed sulphate of barytes (crystals in the form épointée of Haüy). From Devonshire, England.

53 Calcareous concretion. From the Lago de Tartari, near Rome.

54. Carbonate of lime, in botryoidal concretions.

55 Botryoidal magnesian limestone. From Durham.

56 Fibrous limestone; edges polished.

57 Fibrous sulphate of lime. Derbyshire, England.

58 Fibrous gxpsum. Nova-Scotia.

59 Compact Fluor spar.

60 Crtstallized fluor spar, polished.

61 Crystallized fluor, on blende. Matlock, England.

62 White crystallized fluor, upon zine ore. Derbyshire, England. 
63 Fluor- spar, crystallized in cubes of a beautiful bluish tint. From Cumberland, England.

64 Fituor spar, crystallized in cubes of a beautiful green. Cumberland.

65 FluOR SPar, crystallized in cubes; colorless.

66 FLUOR SPAR, incrusted with crystals of carbonate of lime.

67 Veined fluor, polished. Derbyshire.

68 VEINED FLUOR, called by the miners blue john.

69 Grour of QUARTZ CRYSTALS, incrusted with transparent terminated six-sided prisms of heavy spar.

70,71 Two six-sided prisms of QUARTZ, with six-sided pyramids of a beautiful brownish tinge.

72 Pseudonorphous quartz, sprinkled with blende. From Alston, Eng, 73 Talc. 74 Green talc. 75 Black spinelle.

76 BRown GARNET : crystals with twenty trapezoidal faces.

77 GreEN FELSPAR. Siberia.

78 Glassy felspar. Near Bonn.

79 Chrysoberyi. Near Saratoga-springs,

80 Berxl. Acworth, New-Hampshire.

81 BERY (imbedded). Acworth, N. H.

82 Staurotide. Litchfield, Massachusetts,

83 Staurotide. Franconia.

84 Brucite, in carbonate of lime. Warwick, New-York.

85 Onyx ágate. Siberia.

86 Chrysoprase. Baumgarten.

87 Olivine. Habichtwald.

88 Cyanite. Chesterfield.

89 Asbestus. Greenwood furnace, Orange county, New-York.

90 GoLd ore. Charlotte, North-Carolina.

91 Silver oRe. Siberia.

92 Muriate of silver. Siberia.

93 Copper ore. Franklin, New-Jersey.

94 Arseniate of COPPER, on arsenical iron. Amity, New-York.

95 RED OXIDE of COPPER. Siberia.

96 RED OXIDE of COPPER, incrusted with green carbonate of copper and quartz. Siberia.

97 Blue carbonate of coprer. Siberia.

98 Malachite. Siberia.

99 VEINS of LEAD ORE. From the Odin mine;

100 Slickenside galena. From the Odin mine.

101 Arseniate of Lead. From Caldbeck-fells, Cumberland.

102 Arsenicat pyrites? incrusting quartz erystals. Caldbeck-fells. 
103 Molyrdena. Caldbeck-fells.

104 Uranite. Cornwall, England.

105 Oxide of tiN. Cornwall.

106, 107 Axinite. Cornwall.

108 Volcanic basalt. Cassel.

109 Mineral caoutchouc. England.

110 Elastic bitumen: 111 Carbonate of lead.

112 Sulphuret of zinc and tin. Hartz, Germany.

113 Cristallized brown oxide of iron. Cornwall.

114 Massive garnet. 115 Semiopal.

116 Porphyry, containing bronzite.

117 Lepịnolip̣e and rubellite. Pạis, Maine.

118 Drusy quartz.

119 Auriferous pyrites. North-Carolina.

\section{FOSSILS,}

120 Calynene senaria (folded), Hall. Trenton limestone:

121 Head and post-abdomen of Calymene senaria, Hall. Trenton limestone.

122 Cephalic shield of Trinucleus concentricus, Hall. Trenton limestone.

123 Matrix of Calymene senaria, Hall. Trenton limestone?

124 Brelierophon bilobatus, var. corrugatus, Hall. Trenton limestone. 125, 126, 127 Chetetes lycoperdon, Hall. Trenton limestone.

128 Calymẹne blumenbachif (in two positions), Brongniart. Dudley, England.

129 A SLAB covered with small bivalves, fragments of crustaceans, corallincs, etc. From Dudley, England.

130 Post-ABdomen of an Asaphus (not described by Murchison). Dudley. 131 Euomphajus pentangularis. From the Mountain limestone of Semplough, Cumberland, England.

132 Spirifer obtusis. From Egalsfield, Cumberland.

133 Sptrifer attendatus. From Semplough, Cumberland.

134, 135 Bivalve (Genus Allorisma of King). From Parkhead, Cumberland.

136 Spirifer glaber. From the Mountain limestone of England.

137 Spirifer lynx. Mountain limestone of England.

138, 139 Orthis resupinatus. Mountain limestone of England.

140 Favosites (polished). Mountain limestone. 
141 Spirifer. Mountain limestone of England.

142 Acroculia. From Oriskany sandstone.

143, 144 Cornulites arcuatus. Niagara limestone.

145 Cast of Leptena. From Schoharie grit.

146 Crinoidal colvms. Mountain limestone of England.

147 Coralline (silicified). Corniferous limestone.

148 Encrinal, marble (polished). Derbyshire, England.

149 Encrinal marble (polished). Peak Castle, England.

150 Pecten? From the top of one of the mountains of the Jura chain, Switzerland.

151, 152 Calanites. Cumberland, England.

153 Calamites —? Workington, Cumberland.

154 Calamites —? Parton, Cumberland.

155 Stigmaria —. Cumberland.

156, 157, 158 Lepidodendron —. Cumberland.

159 Odontopteris —. Parton, Cumberland.

160 Neuropteris —. Somerset, England:

161, 162 Pecopteris mantellit. Near Whitehaven, Cumberland.

163 Odontopteris —. From Whitehaven, Cumberland.

164 Sphenopteris AFFinis? Whitehaven.

165, 166 Sphenopteris —. Whitehaven.

167 Asterophyllites parkinsoni. Parton, Cumberland,

168 Asterophylutes - Parton, Cumberland.

169,170 Vertebra of the Ichthyosaurus. Lyme in Dorsetshire, Eng. 171 Left hind-paddle of the Ichthyosaurus platyodon. From the Lias, England.

172 Right fore-padde of Ichthyosaurus platyodon. From the Lias, England.

173 Coprolite. One of the sides is polished, exhibiting a fish-scale, and the enamelled portions of fishes that remain undigested by the Ichthyosadrus. From the Lias, England.

174 Fossil Fish. Scotland.

175, 176 Fossil teeth of Ichthyosaurus. From the Blue Lias of Lyme, Dorset, England.

177 Ammonites obtusus. From the Lias, England.

178 Ammonites nodosus? From the Lias, England.

179, 180 Ammonites. Matlock, England.

181, 182, 183 Ammonites. From the Lias, England.

184 Аmmonite. From Whitby, England.

185, 186 Gryphea Incurva. Clifton, Somerset, England.

187 Gryphefa colunba. Beds of the Tiverlane:

$$
5=3
$$


188 Ammonite. From the Oolite, England.

189 Grурнан? (13 specimens), Lias? England:

190 Shark's тоотн, From the Oolite, England:

191, 192 Terebratuta. From the Oolite, England.

193 IchthYODORULITE (mineralized by sulphuret of iron). From the Lias, England.

194 Shark's тоотн (imbedded in chalk). From the Cretaceous formation, England.

195 Turrelites costatus. Chalk marl.

196 Belemites mucronatus, Cretaceous formation, Yorkshire, England. 197 Exogrra costata, From the Greensand, New-Jersey, 198, 199, 200 Gryphea mutabilis. From the Greensand, New-Jersey. 201 GaLerites. From the Cretaceous formation, Yorkshire, England. 202 Ananchytes ovatus. From the Cretaceous formation, Yorkshire. 203 Scutelda. From the Cretaceous formation, England. 204 Murex (Fusus) contrarius, Sowerby. Given to H. C. De Rham junior, by Prof. SEḌGwICK, at the Woodwardian Museum, Cambridge, March 6, 1837. From the Rederag, England. 205 Fusus (covered with BaLanex); From the Redcrag, England. 206 Fossil wood. From Alabama. 207 Petrified wood. From near Utica. 208 Nautilus truncatus. From the Lias of England. 209 Cast of Pleurotomaria. Lias, England. 210, 211 Casts of a dnivalve. Lias, England,

\section{ZOOLOGICAL FRAGMENTS.}

212, 213 Sroll of the Skonk (Mephitis americana).

214 Skull of the Fisher (Mustela canadensis).

215, 216 Skold of the AMerican Sable (Mustela martes).

217 Skoll of the North-American Otter (Lutra canadensis).

218 Upper mandible of the Albatross. From South America.

FISHES.

ORDER PLECTOGNATHI.

FAMTLY GYMNODONTIDA.

219 Diodon pitosus (Hairy Balloonfish). New-York bay. 


\title{
FAMILY OSTRACIONIDAE.
}

220 Lactophrys derhaniI? Taken on the shore of Long Island. Not described in Dekay's Zoology:

Charactertstics. No orbital spines; back elevated in the form of a crescent longitudinally, with two spines centrally situated on the periphery of the crescent, and distant one-third of an inch from each other; three spines on each side of the abdomen: length four inches.

Should this prove to be an undescribed species, it is proposed to name it derhamii, in honor of the late HENRY C. DE RHAM junior.

\section{SHELLS.}

[Arranged according to the System of LAMARCK.]

\author{
CLASS ANNELIDES. \\ ORDER SEDENTARIA. \\ FAMILY SERPULACEA.
}

221, 222, 223 Serfula vermictlaris.

\section{CLASS CIRRIPEDES.}

\section{ORDER SESSILE CIRRIPEDES.}

224 Trbicinella bal.enarum. The Tubicinella are found with nearly the whole shell buried in the thick skin of the whale.

225 Coronula bai.enaris.

226 Coronula balenaris ( 6 specimens), on a piece of whaleskin.

\section{CLASS CONCHIFERA.}

\author{
ORDER C. DIMYARIA.
}

FAMILY CONCHACEA (marine).

227 Cytherea maculata.

228 Cytherea Dione.
229 Cytherea

230 Cytherea

\section{FAMILY CARDIACEA.}

231 Cardium

232 CardiUm
233 CaRDIUM

234 IsOCARDIA MOLTKIANA. 
FAMILY NAJ.ADES:

237 PinNa squamosa?

ORDER MONOMYARIA.

FAMILY MYTILACEA.

HAMILY PECTINIDES.

238 PecteN —.

239 PECTEN —.

\section{CLASS MOLLUSCA.}

ORDER GASTEROPODA.

FAMILY PHYLLIDINA.

240 Chiton $-(5$ specimens $) .241$ Chiton $\longrightarrow$.

242 Chiron -..

243 Bulla ampulla.

FAMILY BULLAEANA.

ORDER TRACHELIPODA.

FAMILY COLIMACEA.

244 Helix pomatia. England. 251 Helix —. West Indies.

245 Helix aspersa(3). England. 252 Carocolla —. West Indies. 246 Helix melanotragus. Africa. 253, 254 Bulinus rosaceus. W. I. 247 Helix nemoralis. England. 255 Bulinds rosaceus (young). 248 HEtix —(2). England. 256, 257 PopA —. West Indies. 249 Helix - West Indies. $25 S$ Auricola - West Indies. 250 Helix —. West Indies.

FAMILY LIMNEANA.

259 LimN五a STAGNALIS. 260 Planoreis corneus.

FAMILY PERISTOMATA.

261 Ampullaria fasciata(3), 262 Ampuldaria guinaica. W. I. 263 Paludina (2).

264 Neritina spinosa.

265 NeritiNa -

266 Natica Mamilla(2).

267 NATICA -

268 Natica

\section{FAMILY NERITACEA.} 269 Natica (2). 270 Nerita peloitonta(2). 271 Nerita vêRSICoĹr. 272 Nerita - 


\section{FAM̆ILY MACROSTOMATA.}

273 Sigaretus concavus.

274 Haliotis tuberculata.
275 HaLiotis CANALICULATA。

276 Haliotis $-(5)$.

\section{IAMILY TURBINACEA.}

277 Solariứir granulatuin.

278 Rotella (not figured or described by Kiener). Seas of Tropical climates.

279 Rotelta (not figured or described by Kiener).

280 Rotelia (not figured or described by Kiener). The three preceding species would be considered by Kiener as varicties of Rotella lineolata.

281 Trochus maculatus.

282 Trochus

(2).

283 Trochus

284 MARGARITA
285 MaRGARItA —.

286 Planaxis —.

287 TURво —.

288 Turbo —.

289 Turritella duflicata; Ceylon.

290 Turritella cingulata, Sowerby. Pacific ocean.

\section{FAMILY CANALTFERA.}

291 Cerithium tuberculatum. 302 Midrex radix(3).

292 Turbinela corniger.

303 Murex brandaris(2)。

293 Turbinella polygona:

304 Murex pinnatus, Swainson،

294 Fasciolaria aurantiaca?

305 Murex elongatus.

295 Fusus monio (varietas):

296 Pyruza melongena.

306 Murex erinaceus.

297 Pyrula carica.

307 Murex calcar, Kiener.

308 Murex crassillabrum? Gray.

298 Pyrula melongena (varietas). 309 Triton variegatum.

299 Ranella beckit, Kiener.

310 Triton succinctufi.

300 Murex regius(4).

301. Murex brasista(2).

311 Triton succinctum (young shell).

\section{FAMILY ALATAE.}

312 Rostellaria pes-Peltcani(2). 317 Strombus gracilio, Sowerby. 313 Strombus lentiginosus(2). 318 Strombus gibberulus. Molucca.

314 Strombus canarium.

315 Strombus Pugilis $(2)$.

319 Strombus floridus. Moluccas. 316 Strombus Grandlatus, Sowerby. 
FAMTLY PURPURIFERA.

321 Casstdaria -

322 Cassis testiculus(3).

323 Cassis erinaceus.

324 Ricinula horrida.

325 Purpura hemastoma(2).

326 Pugfura marginalba, Blainville ; varietas, Kiener.
332 Purpura chocolatum, Duclos.

333 Purpura serturi(2).

334 Monoceros crassilabrum.

335 Monoceros -

336 Dolium variegatum (young). .

337 Doliun variegatum.

338 Dolium perdix.

327 Purpura intermedia, Kiener. 339 Buccinum papillosum(7),

828 Purpura deltoidea.

329 Purpura patula.

340 Buccinum undatum(3).

341 Buccinum reticulatum.

330 Purpura textilosa (young). 342 Buccindim quovit, Kiener. 331 Purpura costata, Blainville. 343 Terebra corrulescens(2).

B44 Columelia meleagris, Duclos.

345 Mitra melondiena. 346 Voluta ilusica.

FAMILY CONVOLUTA.

347 Ovulum ovum. From the hole pierced in the outer lip of this shell, it was undoubtedly worn by a native female of one of the South Sea islands as an ear ornament.

348 Cyprea nucleus.Pacific ocean. 368 Oliva angdlata.

349 Cyprata tigrib(3).

350 Cxprea mauritiana.

351 Cypr za histrio(2).

352 Cyprea talpa.

353 Cyrrefa adusta.

354 Cyprea achatina. N.Holland، 374 Oliva subtuata.

355 CypraA LURIDA.

356 Cypr EA Lynx(3).

357 Cyprata CAPUT-SERPENTIS.

358 Cypraza zigzag.

359 Cyprea Cadrica(4).

360 Cyprea VITELLts.

361 Cyprea catineola(2).

362 Crpr ea erosa. Indian ocean. 382 Conus achatinus, Bruguière.

363 Cypr za - (young shell). 383 Conus cotumba, Bruguì̀re.

364 Cyprea rutei(3). N. Holland. 384 Conus monile, Bruguière.

365 Cyprata xanthodon.

366 Oliva porphyria.

385 Conus terebra, Bruguière.

386 Conus verriculum.

367 Oliva inflata(2). 


\section{RADIATA.}

CLASS ECHINODERMAT

387 Asterias actiteata.

389 Echinus SPHÆRA.
388 Asterias (Gomiaster of Agassiz).

\section{CLASS POLYPI,}

390 FUNGIA AGARICIFORMIS. 391 Fungta

392 Oculina ramba. 393 Oculin.a
394 Madrepora muricata.

395 Madrepora

396 NuLLOPORA

397 Spongia - (attached to an ARCA), 


\section{ADDED TO THE COLLECTION DURING THE PRINTING OF THE CATALOGUE.}

1 Didelphus virginiana. American Opossum (female),

2 Putorius novezoracensis. New-York Ermine (spring plumage). Piesented by Herman Wendelin, M.D.

3 VUȚPES FULVUS. Red Fox (male).

4 Hystrix hudsonius. North-American Porcupine (male). Very large.

5 Numenits longirostris. Long-billed Curlew (male).

6 Podiceps rubricoliss. Red-necked Grebe (male).

7 Salamandra subvíolacea. Violet-colored Salamander (male). 







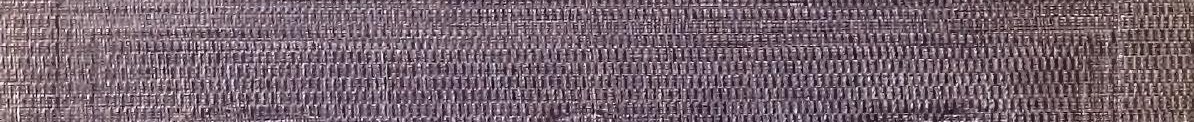

4.

2.

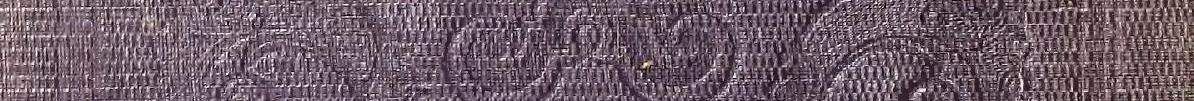

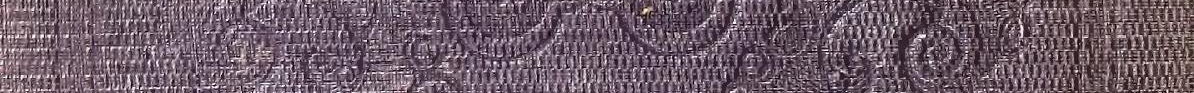

W

H.1.

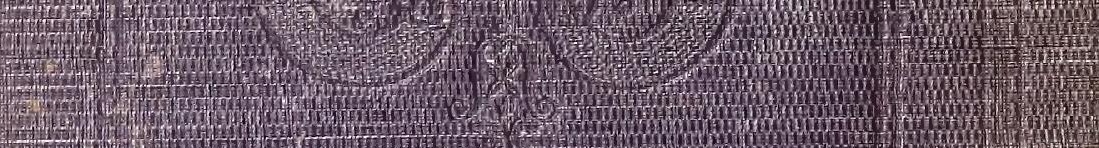

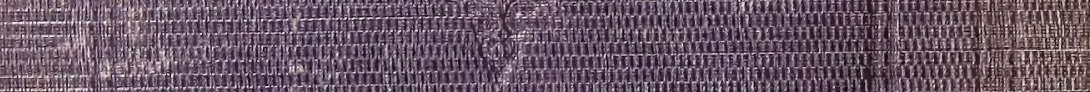

7. 1.

7.7.

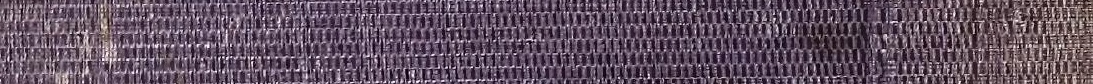

20

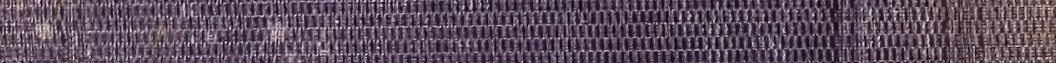

1.1.

4.72

1.

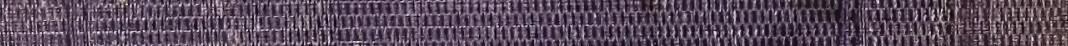

3. 20.

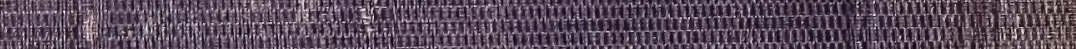

7.

1.7.

H.

4. 1.

(1)

(12)

1.

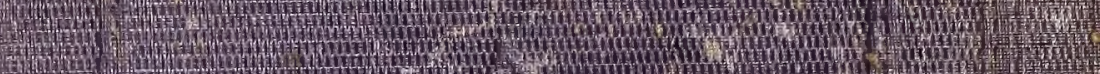

Cot

1.7.

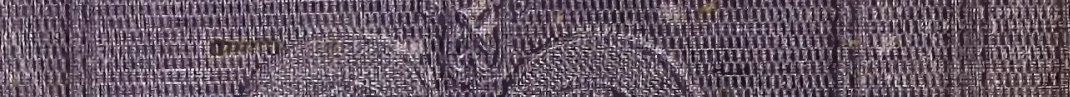

HW

H.1.

H.1.

60.

a

1. 